\title{
Calibration of the MinOS Detectors
}

\author{
Ryan Nichol \\ University College London
}

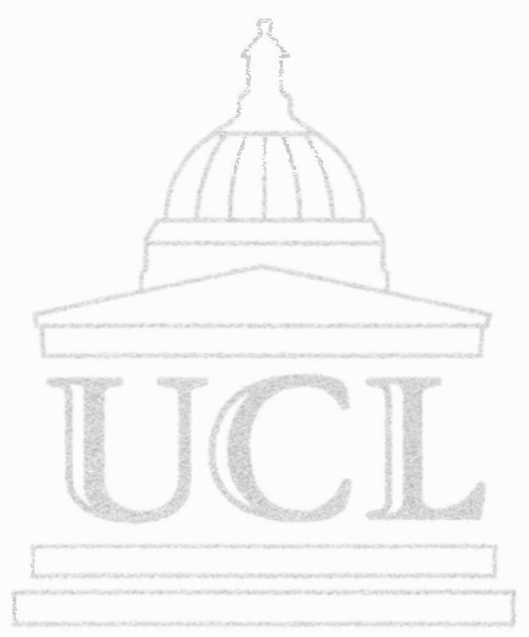

Submitted to the University of London in fulfilment of the requirements for the award of the degree of Doctor of Philosophy.

October 2003. 
ProQuest Number: 10015030

All rights reserved

INFORMATION TO ALL USERS

The quality of this reproduction is dependent upon the quality of the copy submitted.

In the unlikely event that the author did not send a complete manuscript and there are missing pages, these will be noted. Also, if material had to be removed, a note will indicate the deletion.

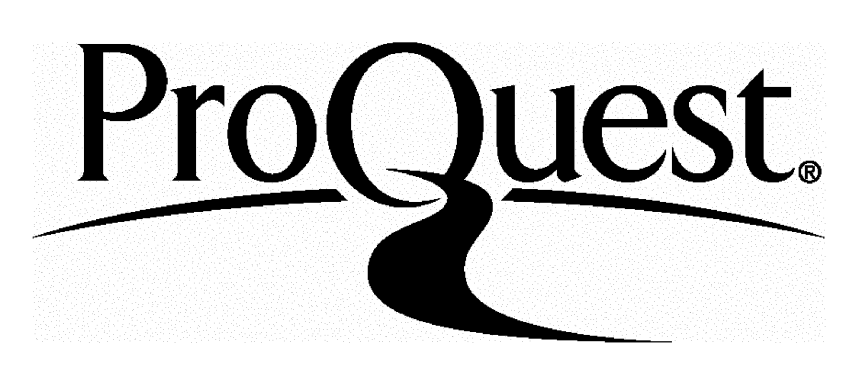

ProQuest 10015030

Published by ProQuest LLC(2016). Copyright of the Dissertation is held by the Author.

All rights reserved.

This work is protected against unauthorized copying under Title 17, United States Code. Microform Edition $\odot$ ProQuest LLC.

\author{
ProQuest LLC \\ 789 East Eisenhower Parkway \\ P.O. Box 1346 \\ Ann Arbor, MI 48106-1346
}




\section{Abstract}

The Main Injector Neutrino Oscillation Search (MINOS) project is a long baseline neutrino oscillation experiment, consisting of a beam of mainly muon neutrinos measured by two steel-scintillator calorimeters. The Near Detector will measure the beam spectrum before oscillations have a had a chance to occur significantly, with the Far Detector measuring the spectrum $735 \mathrm{~km}$ away. MINOS has been designed to achieve a relative energy scale calibration between the detectors to $2 \%$ and an absolute calibration to $5 \%$. The relative calibration is performed using muons and the absolute using measurements from testbeams, taken with the purpose built Calibration Detector (CalDet).

Results are presented from tests of the MINOS calibration chain, carried out using data from the CalDet and Far Detector. A strip to strip calibration of both the CalDet and Far Detector has been carried out using muons and the light injection system. This calibration has been performed to the $2 \%$ level at the CalDet. Results are shown of a cross detector calibration using beam related and cosmic ray muons, with momenta selected through range.

Studies of the charge distribution of upward-going muons, which come from atmospheric neutrino interactions in the rock beneath the Far Detector, are presented. MINOS will be the first experiment that has been able to measure the charge of muons produced from atmospheric neutrinos. 


\section{Acknowledgements}

Firstly I would like to thank Jenny for being my supervisor and providing lots of helpful guidance, advice and support during my studies.

The work presented from the Calibration Detector would not have been possible without the hard work and effort of a great team of people with whom I have worked at CERN. Chris Smith provided a large amount of assistance to the the work on strip to strip calibration presented in Chapter 7 .

The analysis presented in Chapter 9 is heavily indebted to the work of Roy Lee, Brian Rebel and everyone associated with MINOS reconstruction software and Far Detector.

I am particularly grateful for all of the help and advice afforded to me by Chris, Leo, Phil, Ruben and Gordon who have all been of enormous assistance to me during the last three years.

During my nomadic life as a PhD student I have had the pleasure of working in a wide variety places with a large number of people, far too numerous to list here. So thank you to all those people with whom it has been a pleasure to share: an office, a trailer, a mine shaft, a coffee, a beer or a kickabout with the football.

Finally I would like to thank my family for all their support during my student days. Needless to say without them I would not be where I am or who I am today. 


\section{Contents}

1 Introduction $\quad 11$

2 Neutrino Theory $\quad \mathbf{1 5}$

2.1 The Standard Model . . . . . . . . . . . . . . . . 15

2.2 Mass in the Standard Model . . . . . . . . . . . . . . . . 18

2.3 Dirac and Majorana Mass . . . . . . . . . . . . . . . . 21

2.4 Neutrino Mass and the Standard Model . . . . . . . . . . . . . . . 23

2.5 Neutrino Oscillations . . . . . . . . . . . . . . . 25

2.5.1 Vacuum Oscillations ............... 26

2.5.2 Neutrino Oscillations in Matter . . . . . . . . . . . 28

3 Experimental Evidence for Neutrino Oscillations 36

3.1 Solar Neutrinos . . . . . . . . . . . . . . . . . . . 37

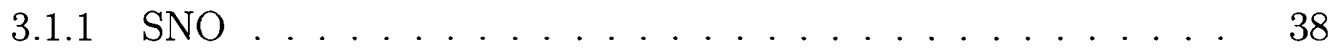

3.2 Atmospheric Neutrinos . . . . . . . . . . . . . . . . . . . 41

3.2 .1 Super-Kamiokande . . . . . . . . . . . . . . . 43

3.3 Reactor Neutrinos . . . . . . . . . . . . . . . . . . 45

3.3 .1 KamLAND . . . . . . . . . . . . . . 46

3.4 Accelerator Neutrinos . . . . . . . . . . . . . . . . . . . . 47

3.4.1 Short Baseline Experiments . . . . . . . . . . . 47

3.4.2 Long Baseline Experiments _. . . . . . . . . . . 48

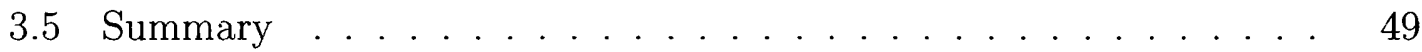


4 The NuMI-MINOS Experiment $\quad 52$

4.1 The NuMI Beam . . . . . . . . . . . . . . . . 53

4.2 The MINOS Detectors . . . . . . . . . . . . . . . . 55

4.2 .1 Generic MINOS Detector . . . . . . . . . . . . 55

4.2 .2 The Far Detector . . . . . . . . . . . . . . . . 57

4.2 .3 The Near Detector . . . . . . . . . . . . . . . . . . . . . 59

4.3 Physics Goals and Capabilities . . . . . . . . . . . . . . 62

$\begin{array}{llr}5 & \text { Overview Of The Calibration } & 68\end{array}$

5.1 Introduction . . . . . . . . . . . . . . . . . . 68

5.2 Why Is Calibration Important? . . . . . . . . . . . . . . . 69

5.3 Relative Calibration .................. 71

5.3 .1 Charge Injection . . . . . . . . . . . 72

5.3 .2 Light Injection . . . . . . . . . . . . . 72

5.3 .3 Strip To Strip Calibration . . . . . . . . . . 74

5.3.4 Cross-Detector Calibration . . . . . . . . . . 75

5.4 Absolute Calibration . . . . . . . . . . . . . . 76

$\begin{array}{lll}6 & \text { The Calibration Detector } & 77\end{array}$

6.1 Why a Calibration Detector? . . . . . . . . . . . . 77

6.2 Description of CalDet . . . . . . . . . . . . 78

6.2.1 CERN Testbeams and Particle Identification . . . . . . . 81

7 The Calibration of the Calibration Detector $\quad 83$

7.1 Light Injection . . . . . . . . . . . . . . . . . 83

7.1.1 Single Photoelectron Peak . . . . . . . . . . . 84

7.1 .2 Linearity . . . . . . . . . . . . . . . . . 98

7.1.3 Drift Point Calibration . . . . . . . . . . . . . . . 103

7.2 Strip to Strip Calibration . . . . . . . . . . . . . . . 111

7.2 .1 Cosmic Ray Muons . . . . . . . . . . . . . . . . 113

7.2 .2 PS Muons in the T7 Testbeam . . . . . . . . . . . . . 136

7.2 .3 Calibration in the T11 Testbeam . . . . . . . . . 142 
7.3 Stopping Muons . . . . . . . . . . . . . . . . . . . . . 143

7.4 Results of Calibration . . . . . . . . . . . . . . 147

7.5 Summary . . . . . . . . . . . . . . . . . 153

8 Cross-Detector Calibration $\quad 154$

8.1 Strip to Strip Calibration of the Far Detector . . . . . . . . . . . 154

8.2 Stopping Muons in the Far Detector . . . . . . . . . . . . . . 157

8.3 Cross-Detector Calibration . . . . . . . . . . . . . . . . 160

8.4 Summary . . . . . . . . . . . . . . . . . . 164

9 Measurement of the Charge Distribution of Atmospheric Neutrino induced Upward-Going Muons $\quad 165$

9.1 Description of Track Fitter . . . . . . . . . . . . . 166

9.1.1 The Least Squares Method . . . . . . . . . . . . . 166

9.1.2 Treatment of Errors . . . . . . . . . . . . 168

9.1.3 Performance of Track Fitter ... . . . . . . . . . 169

9.2 Upward-going Muons at the Far Detector . . . . . . . . . . . . . 177

9.2.1 Selection of Upward-Going Muons . . . . . . . . . . 179

9.2 .2 Results ...................... 184

9.3 Summary . . . . . . . . . . . . . . . . . . . 191

10 Conclusions $\quad 193$ 


\section{List of Figures}

2.1 Conventional and neutrinoless double beta decay . . . . . . . . 24

2.2 Oscillation probability as a function of neutrino energy . . . . . . 27

2.3 Forward scattering of neutrinos in matter via the neutral current . . 29

2.4 Forward scattering of $\nu_{e}$ and $\bar{\nu}_{\mathrm{e}}$ with electrons in matter via the charged current. . . . . . . . . . . . . . . 29

2.5 Resonant enhancement of $\sin ^{2} 2 \theta$ due to the MSW effect . . . . . 33

2.6 Effective $\Delta m^{2}$ due to the MSW effect. . . . . . . . . . . . . 34

2.7 MSW neutrino oscillation probability for the MINOS baseline. . . . 35

3.1 Predicted solar neutrino spectrum with energy thresholds . . . . . . 38

3.2 Neutrino interactions in light water . . . . . . . . . . 39

3.3 Neutrino interactions in heavy water . . . . . . . . . 40

3.4 Comparing solar model predictions to experimental results. . . . . . 42

3.5 Cosmic rays in upper atmosphere eventually producing neutrinos . . 43

3.6 Summary of atmospheric neutrino measurements . . . . . . . . . 44

3.7 Sketch of distance travelled by atmospheric neutrinos. . . . . . . . 45

3.8 Oscillation parameter space for $\nu_{\mu} \leftrightarrow \nu_{\tau} \ldots \ldots \ldots$. . . . . . 50

3.9 Oscillation parameter space for $\nu_{\mu} \leftrightarrow \nu_{e} \ldots \ldots \ldots . \ldots . \ldots 1$

4.1 The path of the NuMI neutrino beam . . . . . . . . . . . 52

4.2 Diagram of the NuMI beamline . . . . . . . . . . 53

4.3 The three configurations of the NuMI beam . . . . . . . . 54

4.4 A short piece of MINOS scintillator . . . . . . . . . . 56

4.5 The completed Far Detector . . . . . . . . . . . . 58 
4.6 The veto shield at the Far Detector . . . . . . . . . . . . . . . 59

4.7 Full and partially instrumented Near Detector planes . . . . . . . . 60

4.8 The instrumented regions of the Near Detector . . . . . . . . . 61

4.9 Sketch of short and long events in the MINOS detectors . . . . . . . 63

4.10 Limit plots for $\nu_{\mu} \rightarrow \nu_{\tau}$ from T-test. . . . . . . . . . . . . . . . . 64

4.11 The oscillated and unoscillated charged current $\nu_{\mu}$ energy spectra. $\quad 65$

4.12 Limit plots for $\nu_{\mu} \rightarrow \nu_{x}$ from charged current $\nu_{\mu}$ interactions . . . 66

4.13 Limit plots for $\nu_{\mu} \rightarrow \nu_{e} \ldots \ldots \ldots$. . . . . . . . . 67

5.1 Schematic diagram of the light injection system . . . . . . . 73

6.1 Photo of the Calibration Detector in T11 testbeam area. . . . . . 79

7.1 Typical low light level M16 spectrum. . . . . . . . . . . . . 85

7.2 Multi-Gaussian fit to low light level spectra . . . . . . . . . 87

7.3 Results from four methods of gain determination. . . . . . . . . . . 90

7.4 Correction factor for gain determination through $(\mu / \sigma)^{2}$ method. . 91

7.5 Simulated M16 ADC spectrum _. . . . . . . . . . . . . . . 92

7.6 Performance of four gain determination methods as function of number of entries. . . . . . . . . . . . . . . 93

7.7 Overestimation of pedestal entries . . . . . . . . . . . . . 95

7.8 Performance of four gain determination methods as function of number of light level. . . . . . . . . . . . . . . . . 96

7.9 The $(\mu / \sigma)^{2}$ method as a function of high light levels. . . . . . . 97

7.10 A light injection linearity curve . . . . . . . . . . . . . . 99

7.11 An example of charge injection . . . . . . . . . . . . . 100

7.12 Non-linear PMT - PIN response at low light level, with blue LED . 101

7.13 Linear PMT - PIN response at low light levels, with ultraviolet LED 102

7.14 Drift point response of single PMT channel over 6 days . . . . . . . 104

7.15 Fractional error on drift point mean . . . . . . . . . . . . 105

7.16 Average gain drift with temperature fluctuations. . . . . . . . . 106

7.17 Histogram of drift point corrections . . . . . . . . . . . 107 
7.18 Drift point response of malfunctioning channel. . . . . . . . . . . 108

7.19 Drift point evolution of linearity curves . . . . . . . . . . . . . 109

7.20 Gain drift corrected to $1 \%$. . . . . . . . . . . . . . 110

7.21 An example muon ADC spectrum . . . . . . . . . . . . . . . 112

7.22 Illustration of PMT crosstalk. . . . . . . . . . . . . . . . . . . 114

7.23 Crosstalk hits along a muon track . . . . . . . . . . . . . . 115

7.24 Example of muon tracking algorithm . . . . . . . . . . . . . 116

7.25 Orientation of scintillator strips . . . . . . . . . . . . . . 117

7.26 The natural angles measured at the CalDet . . . . . . . . . 118

7.27 Angular distribution of cosmic ray muons at CalDet . . . . . . . 119

7.28 Energy deposited as a function of plane by $1.8 \mathrm{GeV}$ stopping beam muons ........................... 120

7.29 Performance of cosmic muon calibration constants . . . . . . . . . . 121

7.30 Sketch of muon path length in scintillator strip . . . . . . . . . . 123

7.31 Corrected and uncorrected mean ADC as function of path length . 124

7.32 Asymmetry in muon response between horizontal and vertical strips 125

7.33 Effect of zero reconstruction on mean of muon ADC spectra . . . 127

7.34 Possible strip orientation bias from incorrect zero reconstruction . . 129

7.35 Alternative zero reconstruction results . . . . . . . . . . . . . 131

7.36 Attenuation in Scintillator Module . . . . . . . . . . . . . . . 132

7.37 Performance of corrected cosmic calibration constants . . . . . . . 133

7.38 Performance of cosmic calibration constants . . . . . . . . . . 135

7.39 A typical PS muon from the T7 area . . . . . . . . . . . . 136

7.40 Angular distribution including PS muons . . . . . . . . . . . 137

7.41 Fraction of zero photoelectron hits . . . . . . . . . . . 139

7.42 Energy deposited as a function of plane for PS muons . . . . . . . . 140

7.43 Performance of PS muon calibration constants . . . . . . . . . . . . 141

7.44 Coverage of the PS muons in the T11 testbeam area . . . . . . . . . 142

7.45 An event display of a stopping $1.8 \mathrm{GeV}$ beam muon . . . . . . . . 143

7.46 Range of stopping beam muons . . . . . . . . . . . . . . 144 
7.47 Total energy deposited by beam muons as a function of range . . . 146

7.48 Results of drift point correction test . . . . . . . . . . . . . . . . . 148

7.49 The PS muon strip to strip calibration constants . . . . . . . . . . . 149

7.50 Calibrated and uncalibrated $1 \mathrm{GeV}$ electron energy . . . . . . . 151

7.51 Relative calibration between $\mathrm{T} 11$ and $\mathrm{T} 7 \ldots \ldots . . \ldots 152$

8.1 Angular distribution of cosmic ray muons at the Far Detector . . 156

8.2 Selection of stopping muons in the Far Detector . . . . . . . . . 158

8.3 A stopping cosmic ray muon in the Far Detector . . . . . . . . . . 159

8.4 A relative calibration between the Far and Calibration Detectors . . 160

8.5 The $\frac{d z}{d s}$ of stopping muons . . . . . . . . . . . . . 162

8.6 Total energy against range for three regions of $\frac{d z}{d s} \ldots \ldots . . \ldots 163$

9.1 The momentum spectrum from Far Detector cosmic ray Monte Carlo 171

9.2 Momentum determination as a function of track length . . . . . . . 172

9.3 Muon momentum determination and charge misidentification . . . 173

9.4 Charge identification efficiency as a function of muon energy . . . . 174

9.5 Measured momentum spectrum of cosmic ray muons in the Far Detector 175

9.6 Fractional error on muon momentum . . . . . . . . . . . 176

9.7 Summary of $\nu_{\mu}$ and $\bar{\nu}_{\mu}$ charged current interaction cross-sections. $\quad 178$

9.8 Distribution of time-y position gradients . . . . . . . . . . . . 181

9.9 Vertical angular distribution of muons . . . . . . . . . . . 182

9.10 The relative efficiency of cuts to select a sample of upward-going muons 183

9.11 An event display showing a upward-going muon in the Far Detector 185

9.12 A second event display showing a upward-going muon in the Far Detector . . . . . . . . . . . . . . . . . 186

9.13 The $\frac{1}{\beta}$ distribution for muons in the Far Detector . . . . . . . . 187

9.14 The flux of upward-going muons as a function of vertical angle . . . 188

9.15 The charge and momentum distribution of atmospheric neutrino induced upward-going muons . . . . . . . . . . . . . . . 190 


\section{Chapter 1}

\section{Introduction}

The Standard Model of particle physics has been outstandingly successful at describing fundamental particles and their interactions. However despite its success it can not be the final theory of particle physics as it does not describe gravity. There are also a number of areas where evidence is growing which can not be fully understood within the theory. One such area is the study of neutrinos.

The standard model contains the 12 fundamental fermions: six quarks and six leptons, along with their antiparticles. It also contains the gauge bosons for the electromagnetic, weak and strong forces. In the model the neutrino is a fermion with zero electromagnetic charge, leaving it only able to interact via the weak interaction. As it is a lepton, it exists in three flavours: electron, muon and tau. In the Standard Model the neutrino is one of only two massless particles, the other being the photon. The photon is required to be massless to explain the long distance nature of the electromagnetic force. There is no similar compelling reason for why the neutrino should be massless. The fact that it is massless does explain the maximal parity violation in the weak interaction, although masses of the $\mathrm{eV}$ scale could also be accommodated within the observed parity violation.

Whilst the neutrino was first postulated by Wolfgang Pauli in 1930 [1], it was not directly observed until 1956 by Reines and Cowan with their experiment at the Savannah River nuclear reactor [2] . The experiments of Wu et al [3] and Goldhaber et al [4], showed that the weak interaction maximally violated parity 
and that the massless neutrino must be lefthanded. Measurements of the width of the $\mathrm{Z}^{0}$ resonance at CERN [5][6][7][8] demonstrated that there were three and only three light neutrinos that couple to the $\mathrm{Z}^{0}$.

A full understanding of neutrinos has proved elusive. There is strong evidence showing a disagreement between the numbers of neutrinos detected at the Earth's surface, compared to predictions from the standard solar model [9]. Experiments, such as SNO [10] and Super-Kamiokande [11], have measured the number of $\nu_{e}$ arriving from the sun, and found there to be fewer than predicted by the standard solar model. The other source of neutrinos which have exhibited anomalous results is from particle showers caused by cosmic rays interacting in the atmosphere. Theoretically one should see about twice as many $\nu_{\mu}$ as $\nu_{e}$. Experiments investigating these atmospheric neutrinos have not observed this ratio, in particular Super-Kamiokande [12] has reported that this discrepancy is due to missing $\nu_{\mu}$.

One possible solution to these problems was originally proposed by Pontecorvo $[13][14][15]$, by analogy to the phenomenon of flavour mixing in the quark system. The eigenstates of the weak interaction are not the same as the mass eigenstates, but are related by the Cabbibo-Kobayashi-Maskawa, or CKM, matrix. If neutrinos are superpositions of mass eigenstates, then they would be created and detected as weak eigenstates, but propagate as mass eigenstates. This could lead to oscillations between the different flavours of neutrinos. In this case the discrepancies in the numbers of neutrinos detected could be explained by assuming that they had oscillated into a different flavour state. This solution would however require the neutrinos to have mass, and a difference in mass between the mass eigenstates.

Possible neutrino mass difference has been investigated by current experiments, such as Super-Kamiokande and SNO, and limits on the mixing parameters have been set. However it is difficult to make precise measurements dealing with solar or atmospheric neutrinos, as the experiments have no control over the creation of the neutrinos. Nuclear reactors are another source of neutrinos which have been used by experiments like KamLand [16] to investigate the phenomenon. The Main Injector Neutrino Oscillation Search, MINOS, experiment is one of a new breed of 
neutrino experiments in which a beam of neutrinos is created at a particle accelerator. MINOS is a long baseline neutrino oscillation experiment, in which a beam of neutrinos is created at the Fermi National Accelerator Laboratory (FNAL), and fired through Earth towards Minnesota. There are two MINOS detectors in which the neutrinos can interact. The Near Detector, being built at FNAL, will sample the beam before oscillations have had a chance to occur significantly. The Far Detector, now installed in the Soudan Mine $735 \mathrm{~km}$ from FNAL, will make a second measurement of the beam. From these measurements the oscillation parameters can be estimated.

Calibration is critical to the determination of the oscillation parameters. The main MINOS measurement relies on comparing the energy spectra of the neutrino beam at the Near and Far Detectors, so it is essential that we understand any differences in the relative response of the detectors. Absolute calibration is important to accurately determine the oscillation parameters. Cosmic ray muons are used for relative calibration, with a light injection system which monitors changes on a short timescale. The absolute calibration is achieved using the third MINOS detector, the Calibration Detector (CalDet). This has the same basic design as the Near and Far Detectors but is smaller. The CalDet is being used in a series of testbeams at CERN to characterize the response of the detectors to hadrons, electrons and muons of known energies. MINOS has been designed to measure the oscillation parameters, $\Delta m^{2}$ and $\sin ^{2} 2 \theta$, to the $10 \%$ level. For this to be possible the relative energy scales of the Near and Far Detectors must be calibrated to within $2 \%$ of each other. The calibration of the absolute energy scale must be carried out at the $5 \%$ level.

The two large MINOS detectors are magnetized, a feature that will enable independent studies of the oscillation characteristics of $\nu_{\mu}$ and $\bar{\nu}_{\mu}$. MINOS will be able to make the first measurement of the charge distribution of atmospheric neutrinos, from the track curvature of neutrino induced muons in the Far Detector.

Chapters 7, 8 and 9 constitute the work that is being presented for this degree. The work on strip to strip calibration in section 7.2 and the selection of electrons 
in section 7.4, were accomplished with the assistance of others. The work presented in Chapters 8 and 9 utilized the MINOS reconstruction software for the purposes of track finding and shower identification. The Monte Carlo events described in Chapter 9 were provided by the collaboration. 


\section{Chapter 2}

\section{Neutrino Theory}

The standard model is perhaps the most successful theory in physics ever developed. It has correctly predicted many observed quantities in the particle physics realm. However, it cannot be the final theory of particle physics as it does not attempt to incorporate gravity. Furthermore, many of the parameters, such as the fermion masses, are arbitrarily set rather than falling out of an underlying principle. In this chapter the central ideas of the standard model will be described, in particular how mass is dealt with. Extensions to the model which could allow neutrino mass will be examined, and the consequences of a massive neutrino will be explored.

\subsection{The Standard Model}

The standard model was created to describe the observed interactions of particles. In the model all observed interactions are described through the interactions of fundamental particles via three forces: the electromagnetic, weak and strong forces. There are two classes of fundamental particle: the fermions, which are the constituents of matter, and the bosons, which carry the three forces.

\section{Fermions}

Fermions are 1/2-integer spin particles. They can be subdivided into leptons (from the Greek lepto, meaning light) which do not experience the strong force, and quarks 
which do. In each of these groups there are six particles, and their anti-particles. These can be characterized as three generations of pairs of particles.

There are two types of lepton, distinguishable by their electromagnetic charge. The first generation is formed by the negatively charged electron $\left(e^{-}\right)$and the neutral electron neutrino $\left(\nu_{e}\right)$. The higher generations differ from the first, by the increased mass of the charged lepton. They are formed by the muon $\left(\mu^{-}\right)$and muon neutrino $\left(\nu_{\mu}\right)$ in the second generation, and the tau $\left(\tau^{-}\right)$and tau neutrino $\left(\nu_{\tau}\right)$ in the third. Both the muon and tau are unstable particles as they can decay into certain lighter fermions. In the lepton sector the generations are arranged as

$$
\left(\begin{array}{c}
\nu_{e} \\
e^{-}
\end{array}\right) \quad\left(\begin{array}{c}
\nu_{\mu} \\
\mu^{-}
\end{array}\right) \quad\left(\begin{array}{c}
\nu_{\tau} \\
\tau^{-}
\end{array}\right) .
$$

All of the quarks carry a fractional electromagnetic charge, the up $(u)$, charm $(c)$ and top $(t)$ quarks have $+2 / 3$ whilst the down $(d)$, strange $(s)$ and bottom $(b)$, have $-1 / 3$ the charge of the electron. Generationally they are arranged as

$$
\left(\begin{array}{l}
u \\
d
\end{array}\right) \quad\left(\begin{array}{l}
c \\
s
\end{array}\right) \quad\left(\begin{array}{l}
t \\
b
\end{array}\right) .
$$

As well as experiencing the electromagnetic and weak forces quarks also interact via the strong force, unlike the leptons. This is because they carry colour which is the charge associated with the strong force. There are three colours which a quark can possess named red, green and blue; anti-quarks can possess anti-red, anti-green or anti-blue. Due to an unusual property of the strong force, quarks are never observed in isolation. Instead they form hadrons which are colourless collections of two or three quarks with integer (or zero) electromagnetic charge. Hadrons formed by three quarks, each with a different colour, are known as baryons, common examples being the proton $(u u d)$ and the neutron $(u d d)$. A two quark hadron, or more correctly a quark anti-quark hadron, is called a meson, an example being the $\pi^{-}(\bar{u} d)$.

\section{Bosons and their forces}

The interactions of fundamental particles in the standard model are described through the exchange of a gauge boson. Each of the three forces has one or more 
bosons associated with it. The properties of the boson exchanged influence the macroscopic characteristics of the force. For instance the range of the force is inversely proportional to the mass of the exchanged boson.

The gauge boson of the electromagnetic force is the massless chargeless photon. As the photon is massless the force has infinite range. The force couples proportionally to charge, so only those particles which have charge experience the force.

There are eight massless gluons which mediate the strong force. Despite the massless nature of gluons, the force has an effective range due to the fact that gluons carry colour, which means gluons can couple to each other and the strong force is non-Abelian. This is the reason why quarks are never seen in isolation, and the origin of the gluon name. When a quark is separated from another quark the potential energy in the bond between them becomes large enough to create quark-anti-quark pairs from the vacuum. This results in hadronization such that no coloured quark compound can exist.

The weak force is the force of most interest to neutrino physicists, as the neutrino possesses neither charge nor colour so can only interact via the weak force. The force is carried by three massive bosons: the $W^{+}, W^{-}$and $Z^{0}$. The $W^{ \pm}$bosons have masses of $80.45 \mathrm{GeV}$ while the $Z^{0}$ has a mass of $91.2 \mathrm{GeV}$. Due to their large masses the force is suppressed at low energy, it was this characteristic that led to the naming of the force.

Weak interactions are peculiar in that they violate parity. Only left-handed fermions and right-handed anti-fermions participate in weak interactions. For the massless neutrino this is a particularly interesting phenomenon. As in the massless limit a left-handed chiral state is equivalent to a left-handed helicity state, where helicity is the projection of the momentum unit vector onto the spin unit vector of a particle. For instance neutrinos produced from $\beta$-transitions of polarized cobalt nuclei in the reaction

$$
{ }^{60} \mathrm{Co} \rightarrow{ }^{60} N i^{*}+e^{-}+\bar{\nu}_{\mathrm{e}}
$$

were observed, indirectly, travelling in a certain direction with respect to the direction of polarisation of the cobalt [3]. If the neutrino were a massive particle it 
could sometimes be measured in a right-handed helicity state and therefore some neutrinos should be observed travelling in the opposite direction. However, neutrinos with a mass of only a few $\mathrm{eV}$ would also be consistent with the observed parity violation.

\subsection{Mass in the Standard Model}

The standard model is an $\mathrm{SU}(3) \otimes \mathrm{SU}(2) \otimes \mathrm{U}(1)$ gauge theory. Before we discuss how mass is introduced in to the standard model it is worth spending some time discussing gauge symmetries and conserved quantities.

In quantum field theory, fermions are described in terms of Lagrangians and field equations. When a local gauge symmetry is imposed upon a fermion field, a conserved quantity can be defined. This conserved quantity is the charge associated with a boson field term which is introduced to preserve the symmetry. The Lagrangian for a free fermion can be written as

$$
\mathcal{L}=\bar{\psi}\left(i \gamma^{\mu} \delta_{\mu}-m\right) \psi
$$

where $\psi$ is the fermion field describing a particle of mass $m$. If one demands a local $\mathrm{U}(1)$ symmetry of the form

$$
\psi \rightarrow \psi^{\prime}=e^{i \alpha(x) Q} \psi
$$

then the Lagrangian for quantum electrodynamics (QED) is obtained:

$$
\mathcal{L}=\bar{\psi}\left(i \gamma^{\mu} \delta_{\mu}-m\right) \psi-e \bar{\psi} \gamma^{\mu} Q \psi A^{\mu}-\frac{1}{4} F_{\mu \nu} F^{\mu \nu}
$$

where $A^{\mu}$ is the field required to preserve the invariance and $F_{\mu \nu}=\delta^{\mu} A^{\nu}-\delta^{\nu} A^{\mu}$. For local gauge invariance the interaction term $-e \bar{\psi} \gamma^{\mu} Q \psi A^{\mu}$ between the fermion field, $\psi$, and the field, $A^{\mu}$ is necessary. In QED $A_{\mu}$ is identified as the photon field and $Q$ is the charge operator, whose eigenvalues are the conserved quantity associated with the symmetry. The strength of the coupling between the fields is determined in part by the magnitude of the conserved quantity, which in this case is the electromagnetic charge. 
The electromagnetic and weak forces are unified in electroweak theory by the imposition of $\mathrm{SU}(2)_{L} \otimes \mathrm{U}(1)$ symmetry. If one requires this gauge invariance four fields are introduced; the interaction terms introduced to the Lagrangian are

$$
-g \overline{\chi_{L}} \gamma_{\mu} \mathbf{T} \cdot \mathbf{W}^{\mu} \chi_{L}=g^{\prime} \bar{\psi} \gamma_{\mu} \frac{Y}{2} \psi B^{\mu}
$$

where $\mathbf{W}^{\mu}$ and $B^{\mu}$ are the vector fields introduced to preserve the gauge invariance. The operators $\mathbf{T}$ and $Y$ are the operators whose eigenvalues are conserved quantities governing the strength of the coupling.

We know the $W^{ \pm}$only couple with left-handed fermions, therefore the fermion fields are separated into left and right chiral projections. The left-handed fields must form $\mathrm{SU}(2)$ doublets, for the first generation of leptons and quarks these are

$$
\chi_{L}^{l}=\left(\begin{array}{c}
\nu_{e L} \\
e_{L}
\end{array}\right) \quad \text { and } \quad \chi_{L}^{q}=\left(\begin{array}{c}
u_{L} \\
d_{L}
\end{array}\right)
$$

the right-handed fields form $\mathrm{SU}(2)$ singlets $e_{R}, u_{R}$ and $d_{R}$. Note that there is no right-handed neutrino state in the standard model.

Although we have four fields represented by four gauge bosons, these bosons are not the $W^{ \pm}, Z$ and $\gamma$ bosons described earlier. Due to the requirements of gauge invariance and theory renormalization, the four bosons must be massless. A separate process is necessary to obtain the familiar bosons and to generate mass for the $W^{ \pm}$and $\mathrm{Z}$ bosons. This process is the Higgs mechanism.

Underlying the Higgs mechanism is the concept of spontaneous symmetry breaking. We consider four scalar particles, $\phi_{i}$, with a $S U(2) \otimes U(1)$ gauge invariant Lagrangian given by

$$
\mathcal{L}=\left|\left(i \delta_{\mu}-g \mathbf{T} \cdot \mathbf{W}_{\mu}-g^{\prime} \frac{Y}{2} B_{\mu}\right) \phi\right|^{2} .
$$

Now lets assume that the vacuum is not a singlet of the gauge symmetry, but instead there are an infinite number of states with same energy. The process of choosing one of these states is known as spontaneous symmetry breaking, as after this choice $U(1)$ transformations can result in a different lowest energy state. Through this process fields obtain non-zero vacuum expectation values. The Higgs potential is 
an example of this, and can be expressed mathematically as

$$
V(\phi)=\frac{1}{2} \mu^{2} \phi^{2}+\frac{1}{4} \lambda \phi^{4}
$$

with $\mu^{2}<0$ and $\lambda>0$.

If this term is added to the Lagrangian gauge invariance is preserved, but the scalar fields acquire a non-zero vacuum expectation value. Also each field acquires an extra degree of freedom due to the degeneracy of the vacuum states. The Higgs scalar fields can be expressed as an $\mathrm{SU}(2)$ doublet:

$$
\phi=\left(\begin{array}{c}
\phi^{+} \\
\phi^{0}
\end{array}\right)=\frac{1}{\sqrt{2}}\left(\begin{array}{l}
\phi_{1}+i \phi_{2} \\
\phi_{3}+i \phi_{4}
\end{array}\right)
$$

The symmetry is broken by choosing one true vacuum state:

$$
\phi_{0}=\frac{1}{\sqrt{2}}\left(\begin{array}{l}
0 \\
v
\end{array}\right)
$$

with $v=\sqrt{-\mu^{2} / \lambda}$.

Once this choice is made the gauge symmetry is spontaneously broken and the vector fields $\mathbf{W}$ and $B$ become massive. The extra degrees of freedom due to the four degenerate vacua of the $\phi$ fields become the longitudinal polarizations for the $\mathbf{W}$ and $B$ fields. Massless particles can only have transverse polarizations, whereas massive particles can also possess longitudinal polarizations.

The $W_{\mu}^{1}$ and $W_{\mu}^{2}$ now correspond to the familiar $W_{\mu}^{+}$and $W_{\mu}^{-}$gauge fields, while the $Z_{\mu}$ and $A_{\mu}$ fields are obtained from mixing the $W_{\mu}^{3}$ and $B_{\mu}$ fields:

$$
\begin{aligned}
& A_{\mu}=\cos \theta_{w} B_{\mu}+\sin \theta_{w} W_{\mu}^{3} \\
& Z_{\mu}=-\sin \theta_{w} B_{\mu}+\cos \theta_{w} W_{\mu}^{3}
\end{aligned}
$$

where $\theta_{w}$ is the weak mixing angle.

Although the $\mathbf{W}$ and $B$ fields are massive, $A_{\mu}$ is chosen such that the operator associated with it is $Q=T_{3}+\frac{Y}{2}$ and $Q \phi_{0}=0$. The spontaneous symmetry breaking generates mass for the $W^{ \pm}$and $Z$ bosons, but the photon remains massless.

The Higgs scalar fields were introduced to generate mass for the $W^{ \pm}$and $Z$ bosons, we can also consider its interactions with the fermion fields. It is possible to 
write down the Lagrangian for the Higgs-fermion couplings, which couple the lefthanded doublet to the right-handed singlet fermion fields. For the first generation of leptons and quarks

$$
\mathcal{L}=f^{e} \bar{\chi}_{L}^{l} \phi_{0} e_{R}+f^{u} \bar{\chi}_{L}^{q} \tilde{\phi}_{0} u_{R}+f^{d} \bar{\chi}_{L}^{q} \phi_{0} d_{R}+h . c .
$$

or

$$
\mathcal{L}=f^{e} \frac{v}{\sqrt{2}} \overline{e_{L}} e_{R}+f^{u} \frac{v}{\sqrt{2}} \overline{u_{L}} u_{R}+f^{d} \frac{v}{\sqrt{2}} \overline{d_{L}} d_{R}+\text { h.c. }
$$

where $\tilde{\phi}_{0}=i \tau_{2} \phi_{0}^{*}$, and $f^{x}$ are the Yukawa coupling constants for the $x$-Higgs interactions. The fermions, except the neutrino, have acquired mass terms of $f^{x} \frac{v}{\sqrt{2}}$. If we generalize to the case where we have three leptonic generations, then cross-coupling between the generations is possible in equation 2.13 , equation 2.14 contains a $3 \times 3$ matrix like

$$
\mathcal{L}=\left(\begin{array}{lll}
\bar{e}_{L} & \bar{\mu}_{L} & \bar{\tau}_{L}
\end{array}\right)\left(\begin{array}{ccc}
\odot & \odot & \odot \\
\odot & \odot & \odot \\
\odot & \odot & \odot
\end{array}\right)\left(\begin{array}{l}
e_{R} \\
\mu_{R} \\
\tau_{R}
\end{array}\right)+h . c . .
$$

\subsection{Dirac and Majorana Mass}

The mass terms described thus far have been Dirac mass terms, which couple left and right-handed fields together. Dirac mass terms are the only kind of mass term available to charged particles. Neutrinos can also have a Majorana mass term, which couples together a particle with its anti-particle, because they are neutral.

We define the following conventions for the charge-conjugation operator, $C$, and the chiral fields, $\psi_{L}$ and $\psi_{R}$ :

$$
\begin{gathered}
\psi^{C}=C \gamma^{0} \psi^{*}=i \gamma^{2} \psi^{*} \\
\psi_{L}=\frac{1}{2}\left(1-\gamma_{5}\right) \psi, \quad \psi_{R}=\frac{1}{2}\left(1+\gamma_{5}\right) \psi \\
\psi_{L}^{C} \equiv\left(\psi_{L}\right)^{C}=\frac{1}{2}\left(1+\gamma_{5}\right) \psi^{C}=\left(\psi^{C}\right)_{R}
\end{gathered}
$$

Fermion mass terms connect left and right-handed fields, so we right down all of 
the Dirac and Majorana mass terms possible:

$$
\begin{aligned}
& \text { Dirac mass: } \mathcal{L}_{D}=m_{D}\left(\overline{\psi_{L}} \psi_{R}+\overline{\psi_{R}} \psi_{L}\right)=m_{D} \bar{\psi} \psi \\
& \text { Majorana mass: } \mathcal{L}_{a}=m_{a}\left(\overline{\psi_{L}^{C}} \psi_{L}+\overline{\psi_{L}} \psi_{L}^{C}\right)=m_{a} \bar{\chi} \chi \\
& \text { Majorana mass: } \mathcal{L}_{b}=m_{b}\left(\overline{\psi_{R}^{C}} \psi_{R}+\overline{\psi_{R}} \psi_{R}^{C}\right)=m_{b} \bar{\omega} \omega
\end{aligned}
$$

with the fields defined as

$$
\begin{aligned}
& \psi=\psi_{L}+\psi_{R}, \\
& \chi=\psi_{L}+\psi_{L}^{C}, \quad \chi^{C}=\chi, \\
& \omega=\psi_{R}+\psi_{R}^{C}, \quad \omega^{C}=\omega .
\end{aligned}
$$

Note how the Majorana fields are self-conjugating, this is only possible if the particle is uncharged.

The most general mass term occurs when all three of the above fields are present:

$$
\begin{aligned}
\mathcal{L}_{D M} & =m_{D} \overline{\psi_{L}} \psi_{R}+m_{a} \overline{\psi_{L}^{C}} \psi_{L}+m_{b} \overline{\psi_{R}^{C}} \psi_{R}+\text { h.c. } \\
& =\frac{1}{2} m_{D}(\bar{\chi} \omega+\bar{\omega} \chi)+m_{a} \bar{\chi} \chi+m_{b} \bar{\omega} \omega \\
& =\left(\begin{array}{ll}
\bar{\chi} & \bar{\omega}
\end{array}\right)\left(\begin{array}{cc}
m_{a} & \frac{1}{2} m_{D} \\
\frac{1}{2} m_{D} & m_{b}
\end{array}\right)\left(\begin{array}{l}
\chi \\
\omega
\end{array}\right) .
\end{aligned}
$$

This equation can be diagonalized to give eigenvalues of

$$
m_{1,2}=\frac{\left(m_{a}+m_{b}\right)}{2} \pm \frac{\sqrt{\left(m_{a}-m_{b}\right)^{2}+m_{D}^{2}}}{2}
$$

which correspond to the Majorana states

$$
\begin{gathered}
\eta_{1}=(\cos \theta) \chi-(\sin \theta) \omega \\
\eta_{2}=(\sin \theta) \chi+(\cos \theta) \omega
\end{gathered}
$$

We can see that in the most general case the neutrino would be a Majorana particle. For there to be a Dirac neutrino one would have to impose a symmetry that forbade the Majorana coupling.

One interesting consequence of having a Majorana type neutrino is neutrinoless double beta decay. There are a small number of radioactive isotopes that can 
decay by emitting two betas simultaneously, ie two neutrons decay into protons. In conventional double beta decay two neutrinos are emitted with the electrons. If the neutrino had Majorana mass, then the right-handed anti-neutrino emitted by one neutron decay has some probability of being absorbed as a left-handed neutrino in the second decay. This is only possible if the neutrino is Majorana particle, and the neutrino is its own anti-particle. The probability of the emitted neutrino being absorbed as a left-handed particle increases with mass, as the left-handed chiral state becomes a mixture of left- and right-handed helicity states. Both types of double beta decay are shown in Figure 2.1. Several experiments are investigating these decays, looking at the energy spectrum of electrons produced in the decays. In conventional double beta decay some fraction of the energy is carried away by the 'invisible' neutrinos, while in neutrinoless double beta decay the sum of the electron energies would give a delta function. As yet no experiment has seen definitive evidence of neutrinoless double beta decay, although a recent re-analysis of data from the Heidelberg-Moscow experiment has claimed to have seen evidence [17] at the $2.2 \sigma$ level, with a best fit to the neutrino mass of $0.39 \mathrm{eV}$. As yet these findings are are uncorroborated, and have not found wide acceptance within the double-beta decay community.

\subsection{Neutrino Mass and the Standard Model}

The standard model generates fermion mass from the coupling of fermions to the Higgs. There is no right-handed neutrino in the standard model and therefore no neutrino term in equation 2.13 , meaning the neutrino is massless in the model. To generate mass for the neutrino we must add something to the standard model.

There are several additions to the model which could generate mass for the neutrino. The simplest thing to add would be a right-handed neutrino, $\nu_{R}$, which would be "sterile" as it has no gauge interactions available to it. The following 
(a)

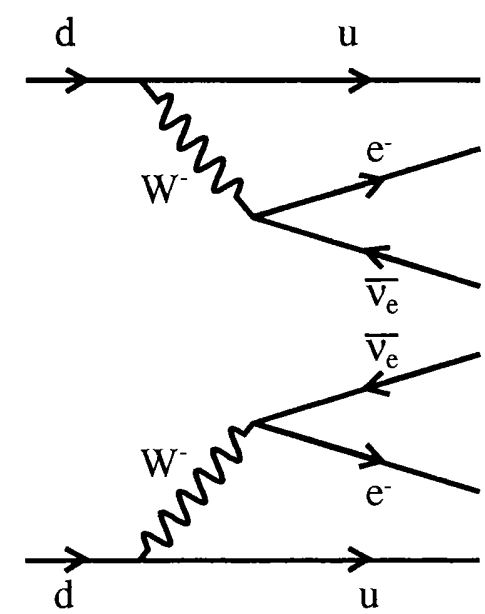

(b)

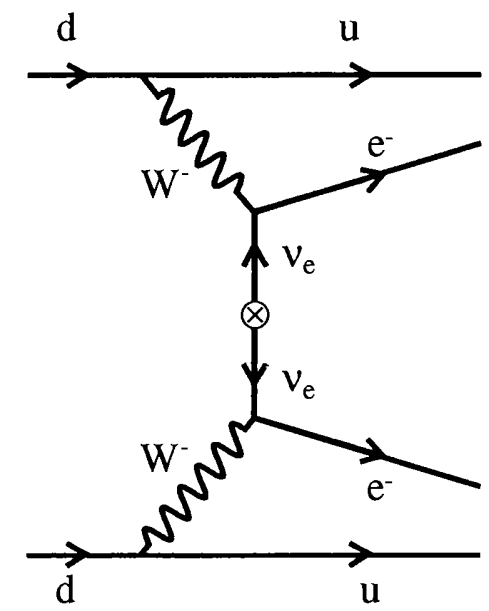

Figure 2.1: On the left is a diagram showing conventional double beta decay where two neutrinos are produced. On the right is neutrinoless double beta decay, the $\otimes$ represents the action of the Majorana mass term.

terms could then be added to the Lagrangian:

$$
\begin{aligned}
\mathcal{L} & =f^{\nu} \overline{l_{L}} \tilde{\phi} \nu_{R}+m_{b} \overline{\nu_{R}} \nu_{R} \\
& =m_{D} \overline{l_{L}} \nu_{R}+m_{b} \overline{\nu_{R}} \nu_{R}
\end{aligned}
$$

The bare Majorana term is allowed for $\nu_{R}$ as it possesses none of the $\mathrm{SU}(2) \otimes$ U(1) quantum numbers. In a unified theory one would like to have a larger group containing the $\nu_{R}$ so the bare coupling $m_{b}$ would come from something like the Higgs symmetry breaking at the unification scale.

From equation 2.27 we now have

$$
\mathcal{L}=\left(\begin{array}{ll}
\bar{\chi} & \bar{\omega}
\end{array}\right)\left(\begin{array}{cc}
0 & \frac{1}{2} m_{D} \\
\frac{1}{2} m_{D} & m_{b}
\end{array}\right)\left(\begin{array}{l}
\chi \\
\omega
\end{array}\right) .
$$

There are three cases to be considered:

$m_{b}=0$ In this situation we recover the Dirac neutrino. A unified theory would need to explain why the neutrino masses are so much smaller than those of the other fermions, and indeed why all the fermion masses are the values that they are. 
$\boldsymbol{m}_{\boldsymbol{b}} \gg \boldsymbol{m}_{\boldsymbol{D}}$ Where we produce one light neutrino with mass $\sim \frac{m_{D}^{2}}{m_{b}}$, which is predominantly $\nu_{L}$, and one heavy neutrino, predominantly $\nu_{R}$, with mass $\sim m_{b}$. This is an example of the seesaw mechanism, which attempts to explain why the neutrino is so light. If $m_{D}$ is of the order of the electron mass, but $m_{b}$ is very large as it results from a Higgs like symmetry breaking at the unification scale.

$\boldsymbol{m}_{\boldsymbol{b}} \sim \boldsymbol{m}_{\boldsymbol{D}}$ The two mass eigenstates have similar masses. In this situation $\nu-\bar{\nu}$ oscillations are possible, this form of oscillation will not be discussed in this thesis. Nevertheless it is worth noting that unlike the atmospheric neutrino experiments, which are not sensitive to sign, MINOS could detect these oscillations as it is magnetized.

\subsection{Neutrino Oscillations}

Now we have seen how a neutrino can acquire a mass term, we will look at the consequences of this addition to the standard model. One of the most interesting consequences is analogous to weak mixing in the quark sector. The weak quark eigenstates are related to the mass eigenstates by the $3 \times 3$ CKM matrix, similarly the weak neutrino eigenstates $\left(\nu_{e}, \nu_{\mu}, \nu_{\tau}\right)$ are related to the neutrino mass eigenstates by a unitary matrix, $U$. For the sake of simplicity only neutrinos with a Dirac mass are considered in this section, the treatment of those with Majorana requires a $6 \times 6$ matrix coupling neutrinos to anti-neutrinos. Neutrinos are produced and observed as weak eigenstates, but they propagate as mass eigenstates; leading to the possibility that a neutrino of type $\nu_{\mu}$ at creation can be observed some time later as type $\nu_{e}$. This phenomenon is known as neutrino oscillation. 


\subsubsection{Vacuum Oscillations}

\section{Two flavour mixing}

In general all three neutrino flavours will mix with each other, however many theoretical models and some experimental observations, favour a dominant mixing mode. This is particularly relevant as experimental evidence, see Chapter 3, suggests that the mixing between $\nu_{e}$ and $\nu_{1}$ is minimal, and the $\nu_{e}$ state is made up of $\nu_{1}$ and a little $\nu_{2}$. It is therefore useful to consider the case where there are two weak neutrino flavours, $\nu_{e}$ and $\nu_{\mu}$, and two mass eigenstates, $\nu_{1}$ and $\nu_{2}$, with masses $m_{1}$ and $m_{2}$. The weak eigenstates are then related to the mass eigenstates by

$$
\left(\begin{array}{l}
\left|\nu_{e}\right\rangle \\
\left|\nu_{\mu}\right\rangle
\end{array}\right)=\left(\begin{array}{cc}
\cos \theta & \sin \theta \\
-\sin \theta & \cos \theta
\end{array}\right)\left(\begin{array}{l}
\left|\nu_{1}\right\rangle \\
\left|\nu_{2}\right\rangle
\end{array}\right) .
$$

A neutrino oscillation experiment, like MINOS, would generate a beam of neutrinos of a certain flavour, say $\nu_{\mu}$, with energy, $E$, at a distance, $L$, from a detector. At the source

$$
\left|\nu_{(x=0)}\right\rangle=\left|\nu_{\mu}\right\rangle=-\sin \theta\left|\nu_{1}\right\rangle+\cos \theta\left|\nu_{2}\right\rangle
$$

After some distance, $L$, the wavefunction is

$$
\left|\nu_{(x=L)}\right\rangle=-\sin \theta e^{i p_{1} L}\left|\nu_{1}\right\rangle+\cos \theta e^{i p_{2} L}\left|\nu_{2}\right\rangle
$$

The probability that at the detector the $\nu_{\mu}$ will have evolved into a $\nu_{e}$ is given by

$$
\begin{aligned}
P_{\nu_{\mu} \rightarrow \nu_{e}} & =\left|\left\langle\nu_{e} \mid \nu_{(x=L)}\right\rangle\right|^{2} \\
& =\left|-\sin \theta \cos \theta e^{i p_{1} L}+\cos \theta \sin \theta e^{i p_{2} L}\right|^{2} \\
& =\sin ^{2} 2 \theta \sin ^{2} \frac{\left(p_{1}-p_{2}\right) L}{2} .
\end{aligned}
$$

But $p_{i}^{2}=E^{2}-m_{i}^{2}$, so in the limit $m_{i}<<E$, which will be true even for a massive neutrino, we can expand $p_{i}=E-\frac{m_{i}^{2}}{2 E}$ and writing $\Delta m_{21}^{2}=m_{2}^{2}-m_{1}^{2}$ we have

$$
P_{\nu_{\mu} \rightarrow \nu_{e}}=\sin ^{2} 2 \theta \sin ^{2} \frac{\Delta m_{21}^{2} L}{4 E}
$$

in natural units. Note that this derivation is a simplification, however the final result is correct. To recover the more familiar two flavour neutrino oscillation formula we 
must express energy in $\mathrm{GeV}$, length in $\mathrm{km}$ and mass in $\mathrm{eV}$ :

$$
P_{\nu_{\mu} \rightarrow \nu_{c}}=\sin ^{2} 2 \theta \sin ^{2} \frac{1.27 \Delta m_{21}^{2} L}{E} .
$$

Using a baseline of $735 \mathrm{~km}$, the MINOS baseline, the oscillation probability as a function of energy for values of $\Delta m_{21}^{2}$ suggested by atmospheric neutrino experiments is shown in Figure 2.2.
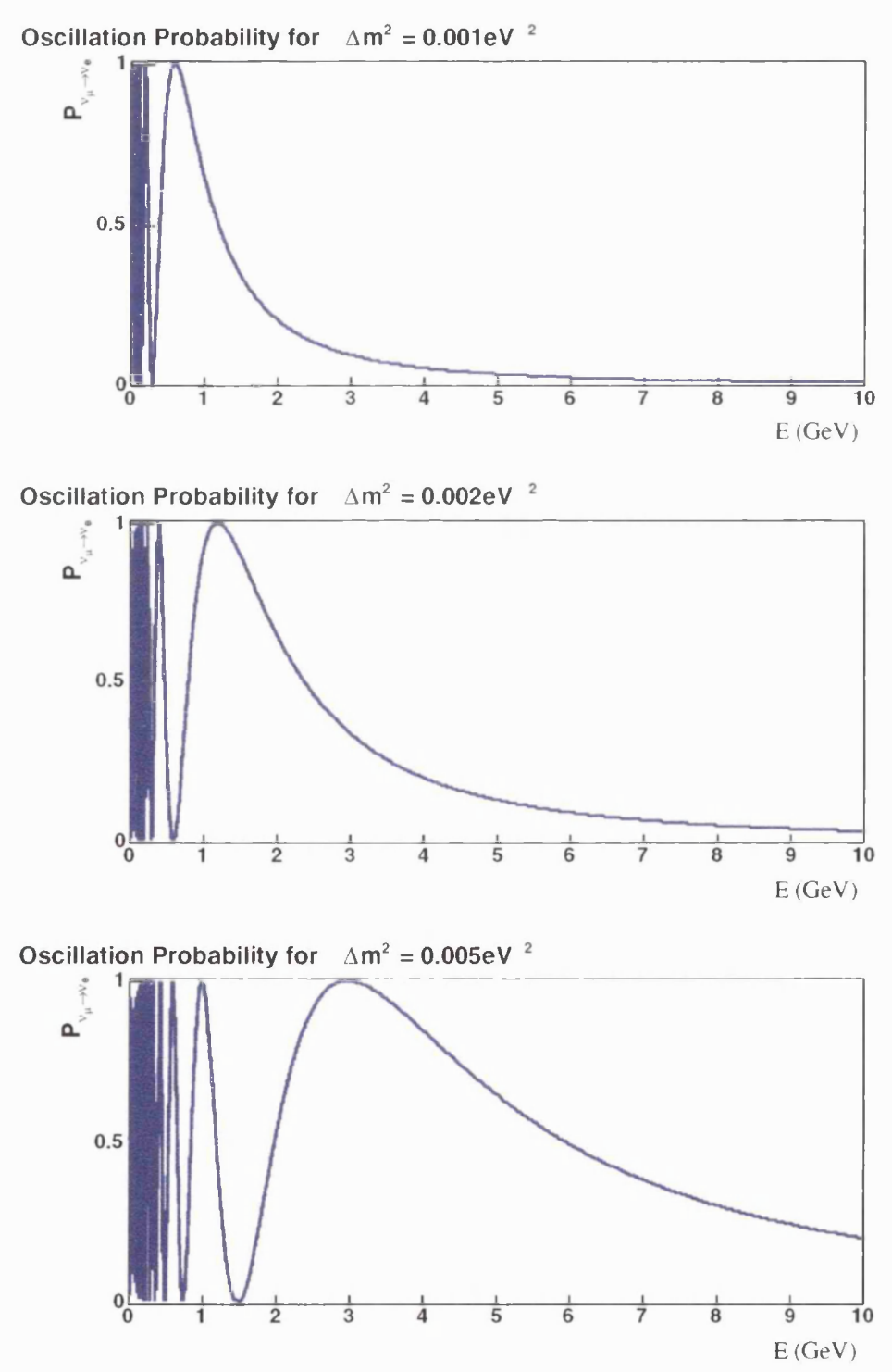

Figure 2.2: Oscillation probability as a function of energy for the two flavour model. Using the MIINOS baseline, $735 \mathrm{~km}$, and $\sin ^{2} 2 \theta=1$. 


\section{Three flavour mixing}

Now if we consider the more general case where there is mixing between three neutrino flavours, we can write the weak eigenstates $\left|\nu_{\alpha}\right\rangle, \alpha \in\{e, \mu, \tau\}$, as linear combinations of the mass eigenstates $\left|\nu_{i}\right\rangle, i \in\{1,2,3\}$ :

$$
\left|\nu_{\alpha}\right\rangle=\sum_{i} U_{\alpha i}\left|\nu_{i}\right\rangle
$$

where $U_{\alpha i}$ are the elements of a $3 \times 3$ unitary matrix, $U$. The initial state is given by

$$
\left|\nu_{(x=0)}\right\rangle=\left|\nu_{\mu}\right\rangle=\sum_{i} U_{\mu i}\left|\nu_{i}\right\rangle
$$

At the detector, a distance $\mathrm{L}$ from the source, we have

$$
\left|\nu_{(x=L)}\right\rangle=\sum_{i} e^{i p_{i} L} U_{\mu i}\left|\nu_{i}\right\rangle
$$

The probability of the neutrino oscillating into a type $\left|\nu_{\alpha}\right\rangle$ is

$$
\begin{aligned}
P_{\nu_{\mu} \rightarrow \nu_{\alpha}} & =\left|\left\langle\nu_{\alpha} \mid \nu_{(x=L)}\right\rangle\right|^{2} \\
& =\left|\sum_{i, j}\left\langle\nu_{j}\left|U_{\alpha j}^{\dagger} e^{i p_{i} L} U_{\mu i}\right| \nu_{i}\right\rangle\right|^{2} \\
& =\sum_{i}\left|U_{\alpha i}\right|^{2}\left|U_{\mu i}\right|^{2}+\sum_{i \neq j} U_{\alpha i}^{*} U_{\alpha j} U_{\mu j}^{*} U_{\mu i} e^{i\left(p_{i}-p_{j}\right) L} \\
& \approx \sum_{i}\left|U_{\alpha i}\right|^{2}\left|U_{\mu i}\right|^{2}+\sum_{i \neq j} U_{\alpha i}^{*} U_{\alpha j} U_{\mu j}^{*} U_{\mu i} e^{i \frac{\Delta m_{j i}^{2}}{2 E}}
\end{aligned}
$$

A standard way to parameterize the matrix $U$ for Dirac neutrinos is

$$
U=\left(\begin{array}{ccc}
c_{12} c_{13} & s_{12} c_{13} & s_{13} e^{-i \delta} \\
-s_{12} c_{23}-c_{12} s_{23} s_{13} e^{i \delta} & c_{12} c_{23}-s_{12} s_{23} s_{13} e^{i \delta} & s_{23} c_{13} \\
s_{12} s_{23}-c_{12} c_{23} s_{13} e^{i \delta} & c_{12} s_{23}-s_{12} c_{23} s_{13} e^{i \delta} & c_{23} c_{13}
\end{array}\right)
$$

where $c_{i j}=\cos \theta_{i j}, s_{i j}=\sin \theta_{i j}, \theta_{i j}$ are the weak mixing angles and $\delta$ is the CPviolating phase.

\subsubsection{Neutrino Oscillations in Matter}

In the case of oscillations in vacuo small mixing angles will always result in small oscillation probabilities. However, if one considers the effect of neutrinos passing through matter the probability can be greatly enhanced. This effect was first 
considered by Wolfenstein [18] and Mikhiyev \& Smirnov [19][20], and is known as the MSW effect. As neutrinos propagate through matter they can undergo elastic forward scattering, such that their momentum does not change. All, non sterile, neutrinos interact via the neutral current channel in Figure 2.3. Electron neutrinos

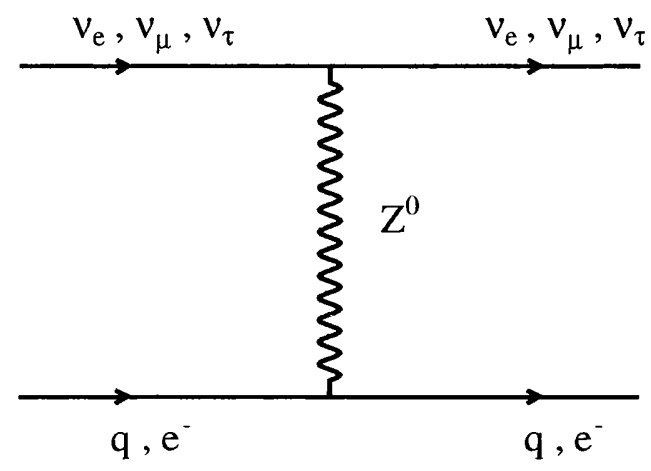

Figure 2.3: Forward scattering of neutrinos in matter via the neutral current. All three neutrino flavours experience these interactions.

and electron anti-neutrinos can also interact through the charge current channels shown in Figure 2.4.

(a)

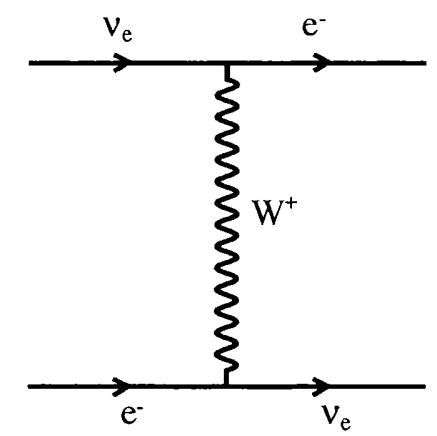

(b)

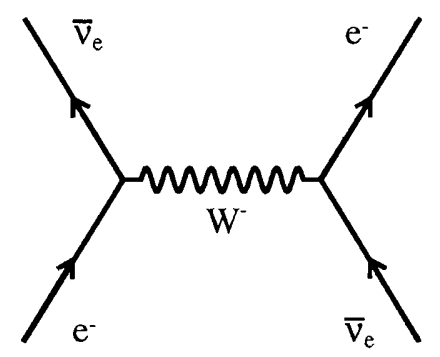

Figure 2.4: Forward scattering of (a) electron neutrino and (b) electron anti-neutrino via charged current interactions with electrons in matter. 
These interactions with matter produce an effective refractive index given by

$$
n=1+\sum_{\alpha} \frac{2 \pi N_{\alpha}}{p^{2}} f_{\nu \alpha}
$$

where $f_{\nu \alpha}, \alpha \in\{e, n, p\}$, is the forward scattering amplitude for the neutrino interacting with particle type $\alpha$ and $N_{\alpha}$ is the number density of that particle. All of the contributions due to neutral current interactions can be ignored for the purpose of neutrino oscillations as they just introduce an overall phase, because all flavours of neutrino couple equally to the $Z$. The charged current interactions, which are only present for electron neutrinos and electron anti-neutrinos, contribute

$$
f_{\nu e}= \pm \frac{G_{F} p}{\sqrt{2 \pi}}
$$

where $G_{F}$ is the Fermi constant and the plus sign is taken for neutrinos and the minus sign for anti-neutrinos. The effect of a refractive index, $n$, is to introduce an extra phase factor of $e^{i(n-1) p x}$. So the interactions with matter produce an additional phase of $\pm \sqrt{2} G_{F} N_{e} x$.

We now consider the effect of this phase on neutrino oscillation, considering only the two flavour model for simplicity. The evolution of the mass eigenstates can be expressed as

$$
-i \frac{\mathrm{d}}{\mathrm{d} x}\left(\begin{array}{l}
\left|\nu_{1}\right\rangle \\
\left|\nu_{2}\right\rangle
\end{array}\right)=\left(\begin{array}{cc}
p_{1} & 0 \\
0 & p_{2}
\end{array}\right)\left(\begin{array}{c}
\left|\nu_{1}\right\rangle \\
\left|\nu_{2}\right\rangle
\end{array}\right)
$$

Or if we express the evolution in terms of the weak eigenstate we have

$$
-i \frac{\mathrm{d}}{\mathrm{d} x}\left(\begin{array}{l}
\left|\nu_{e}\right\rangle \\
\left|\nu_{\mu}\right\rangle
\end{array}\right)=\left(\begin{array}{cc}
p_{1} \cos ^{2} \theta+p_{2} \sin ^{2} \theta & \left(p_{2}-p_{1}\right) \sin \theta \cos \theta \\
\left(p_{2}-p_{1}\right) \sin \theta \cos \theta & p_{1} \sin ^{2} \theta+p_{2} \cos ^{2} \theta
\end{array}\right)\left(\begin{array}{l}
\left|\nu_{e}\right\rangle \\
\left|\nu_{\mu}\right\rangle
\end{array}\right) .
$$

If we now introduce the matter effects, and subtract $p_{1} I$ (which simply contributes a common phase and has no effect on neutrino oscillations), we have

$$
-i \frac{\mathrm{d}}{\mathrm{d} x}\left(\begin{array}{l}
\left|\nu_{e}\right\rangle \\
\left|\nu_{\mu}\right\rangle
\end{array}\right)=\left(\begin{array}{cc}
p_{1} \cos ^{2} \theta+p_{2} \sin ^{2} \theta \pm \sqrt{2} G_{F} N_{e} & \left(p_{2}-p_{1}\right) \sin \theta \cos \theta \\
\left(p_{2}-p_{1}\right) \sin \theta \cos \theta & p_{1} \sin ^{2} \theta+p_{2} \cos ^{2} \theta
\end{array}\right)\left(\begin{array}{l}
\left|\nu_{e}\right\rangle \\
\left|\nu_{\mu}\right\rangle
\end{array}\right) .
$$


This equation has eigenvalues $\lambda_{1}$ and $\lambda_{2}$ with eigenstates $\nu_{1}^{\prime}$ and $\nu_{2}^{\prime}$ which satisfy the following relations:

$$
-i \frac{\mathrm{d}}{\mathrm{d} x}\left(\begin{array}{c}
\left|\nu_{1}^{\prime}\right\rangle \\
\left|\nu_{2}^{\prime}\right\rangle
\end{array}\right)=\left(\begin{array}{cc}
\lambda_{1} & 0 \\
0 & \lambda_{2}
\end{array}\right)\left(\begin{array}{c}
\left|\nu_{1}^{\prime}\right\rangle \\
\left|\nu_{2}^{\prime}\right\rangle
\end{array}\right)
$$

and

$$
\left(\begin{array}{l}
\left|\nu_{e}\right\rangle \\
\left|\nu_{\mu}\right\rangle
\end{array}\right)=\left(\begin{array}{cc}
\cos \theta_{m} & \sin \theta_{m} \\
-\sin \theta_{m} & \cos \theta_{m}
\end{array}\right)\left(\begin{array}{c}
\left|\nu_{1}^{\prime}\right\rangle \\
\left|\nu_{2}^{\prime}\right\rangle
\end{array}\right)
$$

with

$$
\tan 2 \theta_{m}=\frac{\left(p_{2}-p_{1}\right) \sin 2 \theta}{\left(p_{2}-p_{1}\right) \cos 2 \theta \mp \sqrt{2} G_{F} N_{e}} .
$$

We can now follow the argument for two flavour vacuum oscillations and directly write down the probability of a muon neutrino changing flavour:

$$
P_{\nu_{\mu} \rightarrow \nu_{\epsilon}}=\sin ^{2} 2 \theta_{m} \sin ^{2} \frac{\left(\lambda_{1}-\lambda_{2}\right) L}{2} .
$$

It is possible to show that

$$
\lambda_{1}-\lambda_{2}=\frac{\Delta m_{21}^{2}}{2 E} \sqrt{\sin ^{2} 2 \theta+\left(\cos 2 \theta \mp \frac{\sqrt{2} G_{F} N_{e}}{\Delta m_{21}^{2} / 2 E}\right)^{2}}
$$

and also

$$
\sin ^{2} 2 \theta_{m}=\frac{\sin ^{2} 2 \theta}{\sin ^{2} 2 \theta+\left(\cos 2 \theta \mp \frac{\sqrt{2} G_{F} N_{e}}{\Delta m_{21}^{2} / 2 E}\right)^{2}}
$$

by writing

$$
\Delta M_{21}^{2}=\Delta m_{21}^{2} \sqrt{\sin ^{2} 2 \theta+\left(\cos 2 \theta \mp \frac{\sqrt{2} G_{F} N_{e}}{\Delta m_{21}^{2} / 2 E}\right)^{2}}
$$

we now have

$$
P_{\nu_{\mu} \rightarrow \nu_{e}}=\sin ^{2} 2 \theta_{m} \sin ^{2} \frac{1.27 \Delta M_{21}^{2} L}{E} .
$$

At low matter densities $\left(N_{e} \rightarrow 0\right)$ this reverts to the vacuum oscillation probability, whilst at very high densities the oscillations are suppressed by $1 / N_{e}^{2}$. An important facet of MSW oscillations is that one can achieve resonant enhancement of the oscillations, even for small values of the vacuum mixing angle. This resonant enhancement happens when

$$
\frac{\sqrt{2} G_{F} N_{e}}{\Delta m_{21}^{2} / 2 E}= \pm \cos 2 \theta
$$


where the plus sign is taken for neutrinos and the minus sign for anti-neutrinos. So the resonant enhancement is only possible for neutrinos if $m_{2}>m_{1}$ and for anti-neutrinos if $m_{2}<m_{1}$. Figure 2.5 shows the enhancement of the mixing angle for different values of the matter density. Whilst the effective $\Delta m_{21}^{2}$ is shown for the same densities in Figure 2.6. The effect of these on the oscillation probability is shown in Figure 2.7. In each figure three different values of the vacuum mixing angle are shown. The MSW effect has very little influence on the results of the MINOS experiment, due to the relatively low density of the Earth. However, it is important when interpreting the results of solar neutrino experiments, see Section 3.1. 

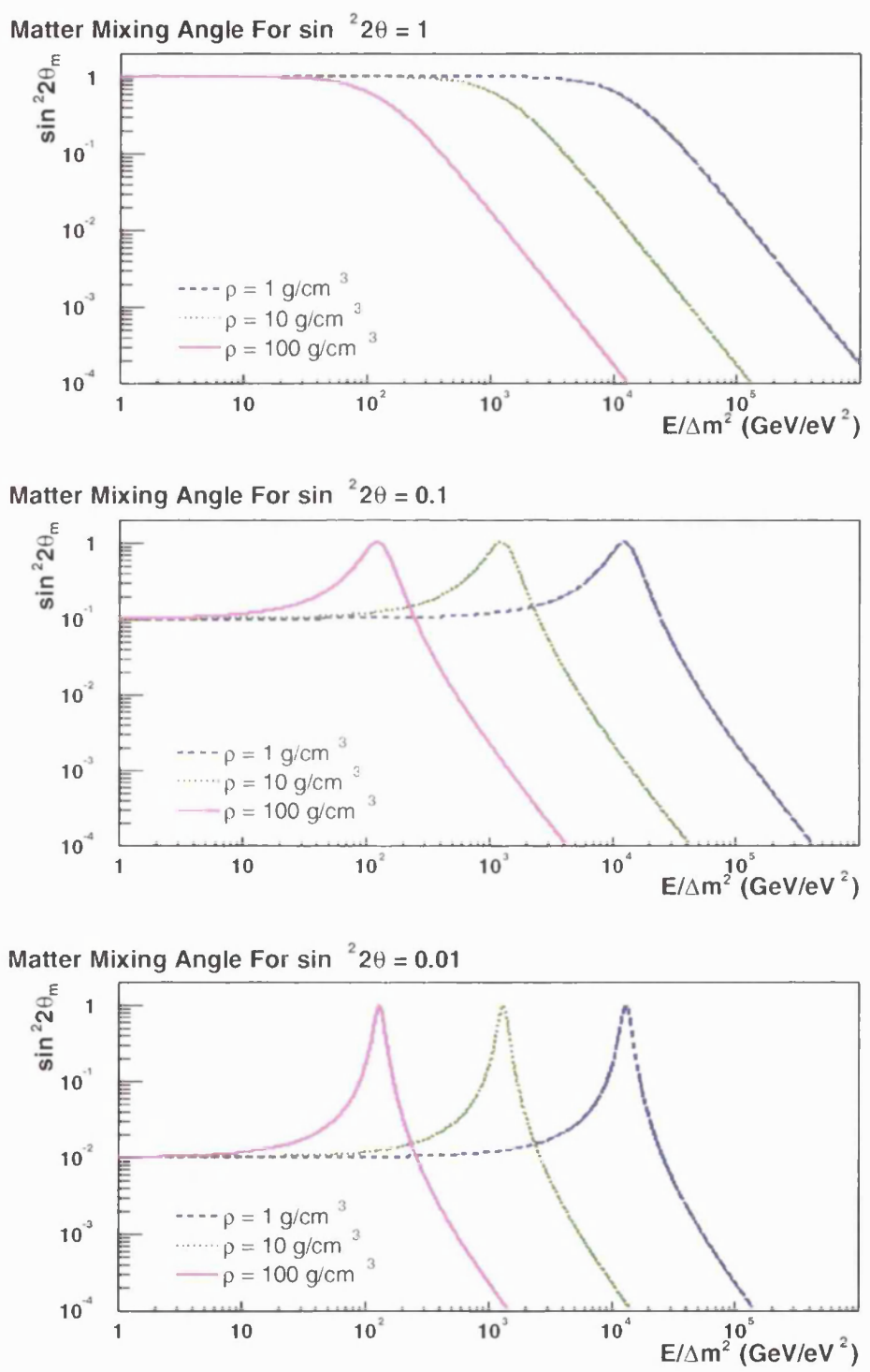

Figure 2.5: The resonant enhancement of neutrino oscillations due to the MSW effect. Interactions with matter can produce an effective maximal mixing. Different values of $\rho$, the matter density, and $\theta$, the vacuum mixing angle are shown. 

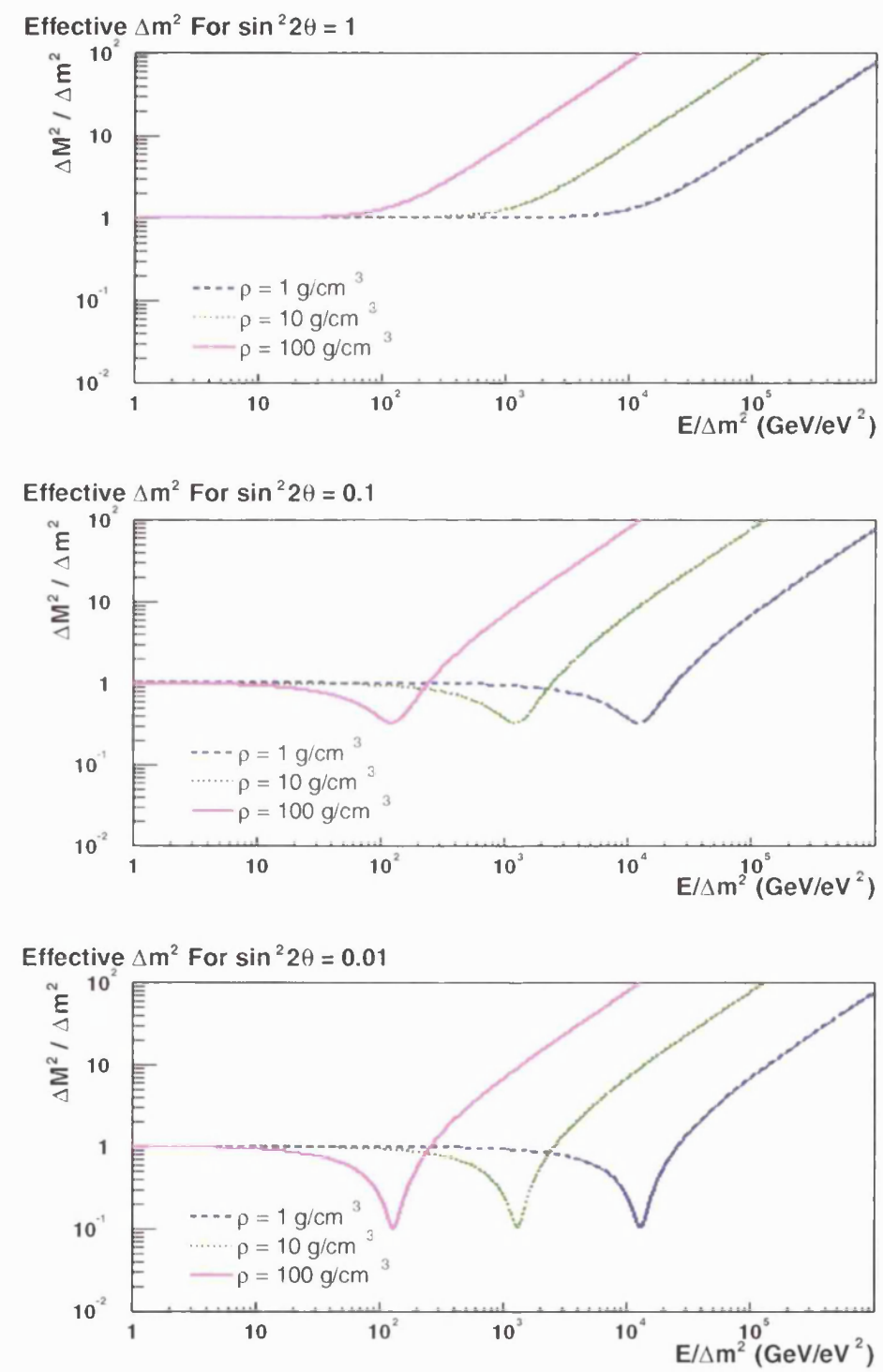

Figure 2.6: The ratio $\Delta M^{2} / \Delta m^{2}$ is plotted for different values of matter density, $\rho$, and $\theta$. Super-Kamiokande predicts $\sin ^{2} 2 \theta \approx 1$ and $E / \Delta m^{2} \approx 10^{3}$ for NuMI neutrinos. In this region there is very little effect on the measurement of $\Delta m^{2}$ due to matter effects. 

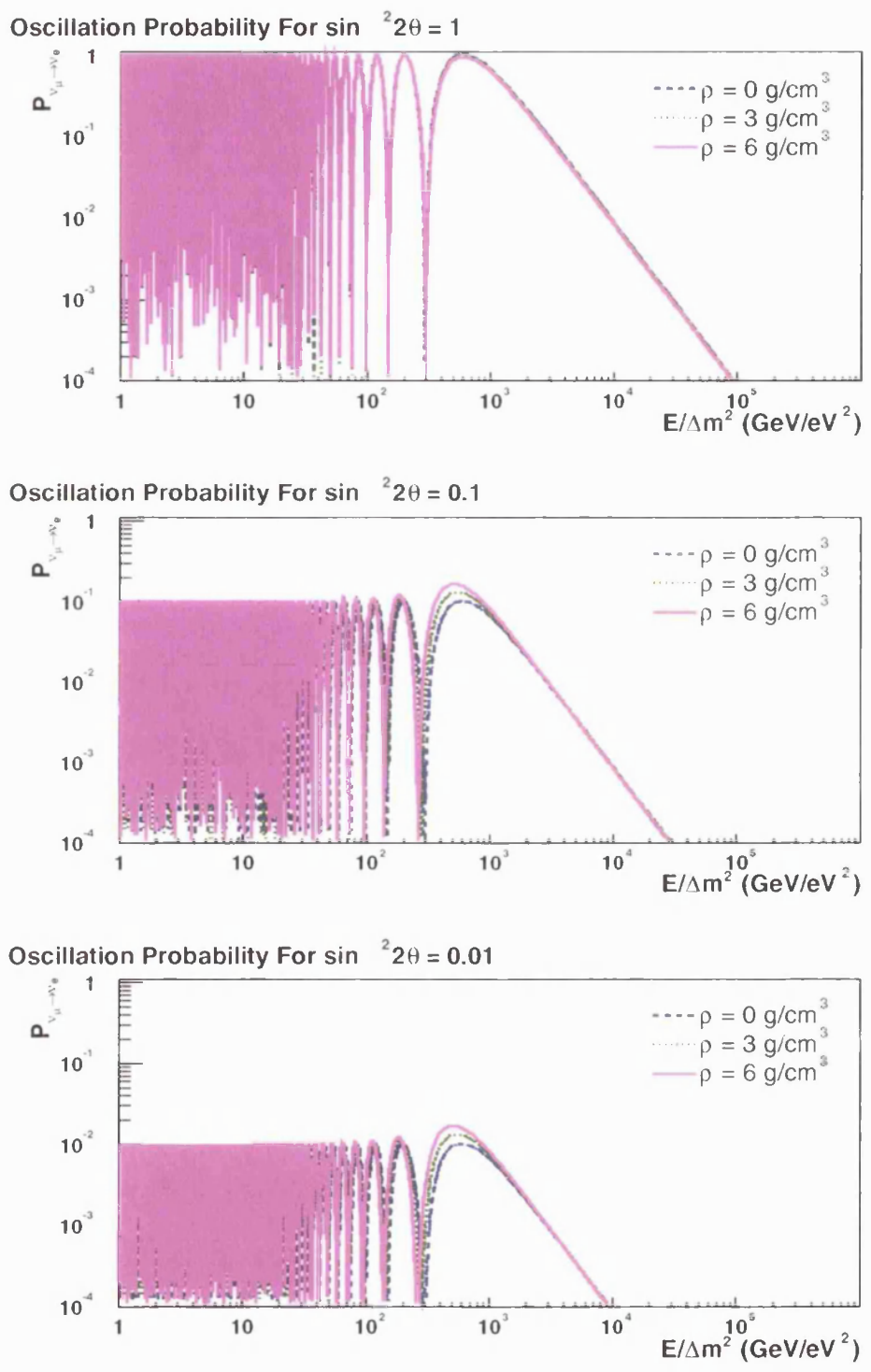

Figure 2.7: The MSW neutrino oscillation probability for the MINOS baseline and different values of $\rho$ and $\theta$. The effects shown in Figure 2.5 and Figure 2.6 work in opposite directions, meaning there is little change to the oscillation probability, for densities around that of the Earth's crust. 


\section{Chapter 3}

\section{Experimental Evidence for}

\section{Neutrino Oscillations}

The first experiment to observe neutrinos from the Sun, conducted by Ray Davis in the Homestake mine [21] in 1968, recorded a deficit of neutrinos compared to theory. Ever since physicists have been looking for the cause of the missing neutrinos. In particular, proof that the missing neutrinos were the result of oscillations has been sought.

There are several sources of neutrino which have been used to investigate oscillations:

The Sun The Sun is a powerful source of neutrinos. They are produced during the nuclear fusion in the core which powers the sun.

The atmosphere Cosmic rays interact with nucleons in the atmosphere. These interactions produce hadrons which decay producing, amongst other things, neutrinos.

Nuclear reactors Electron anti-neutrinos are produced during the $\beta$-decay in nuclear fission processes in the reactors.

Particle accelerators Beams of high energy particles, normally protons, when incident on a target produce a shower of hadrons. These primarily decay into muons and muon neutrinos. 


\subsection{Solar Neutrinos}

Neutrinos from the Sun come from the nuclear fusion reactions, where hydrogen nuclei are fused into helium. There are two cycles through which this process occurs: the pp cycle and the CNO cycle. The dominant process is the pp cycle. In both of these cycles electron neutrinos are created when protons are converted into neutrons. The neutrinos produced have energy of the order of $1 \mathrm{MeV}$.

As mentioned above, the first experiment to detect neutrinos from the Sun was carried out in the Homestake mine in 1968. The mine contained 615 tonnes of tetrachloroethylene, which can interact with neutrinos above an energy of $814 \mathrm{keV}$ via

$$
\nu_{e}+{ }^{37} \mathrm{Cl} \rightarrow e^{-}+{ }^{37} \mathrm{Ar} .
$$

The neutrinos which interact primarily come from the reactions of ${ }^{7} \mathrm{Be}$ and ${ }^{8} \mathrm{~B}$ in the pp cycle, see 3.1 for the solar neutrino spectra and the thresholds of the experiments discussed here. After several weeks of exposure the argon produced was filtered out and the number of atoms counted. Radiochemical experiments of this nature infer the solar neutrino flux from the integrated number of neutrinos detected. The experiment in the Homestake mine measured a deficit between the numbers of neutrinos detected and the predictions of the standard solar model [9].

Since this original measurement there have been several other experiments which have shown agreement with the results from Homestake. SAGE [23][24], GALLEX [25][26] and its successor GNO [27] are all radiochemical experiments that used gallium instead of chlorine, the reaction being

$$
\nu_{e}+{ }^{71} \mathrm{Ga} \rightarrow e^{-}+{ }^{71} \mathrm{Ge} .
$$

The advantage of using gallium is that it has a lower threshold energy of $233 \mathrm{keV}$. This means that it is sensitive to the pp neutrinos, which are the most numerous of the neutrinos produced in the Sun, see Figure 3.1.

The Kamiokande [28] experiment and Super-Kamiokande [11][29][30], its much larger successor, measured a deficit in the solar neutrino flux using a different technique. Kamiokande consisted of a $3 \mathrm{kT}$ tank of purified water surrounded by 980 


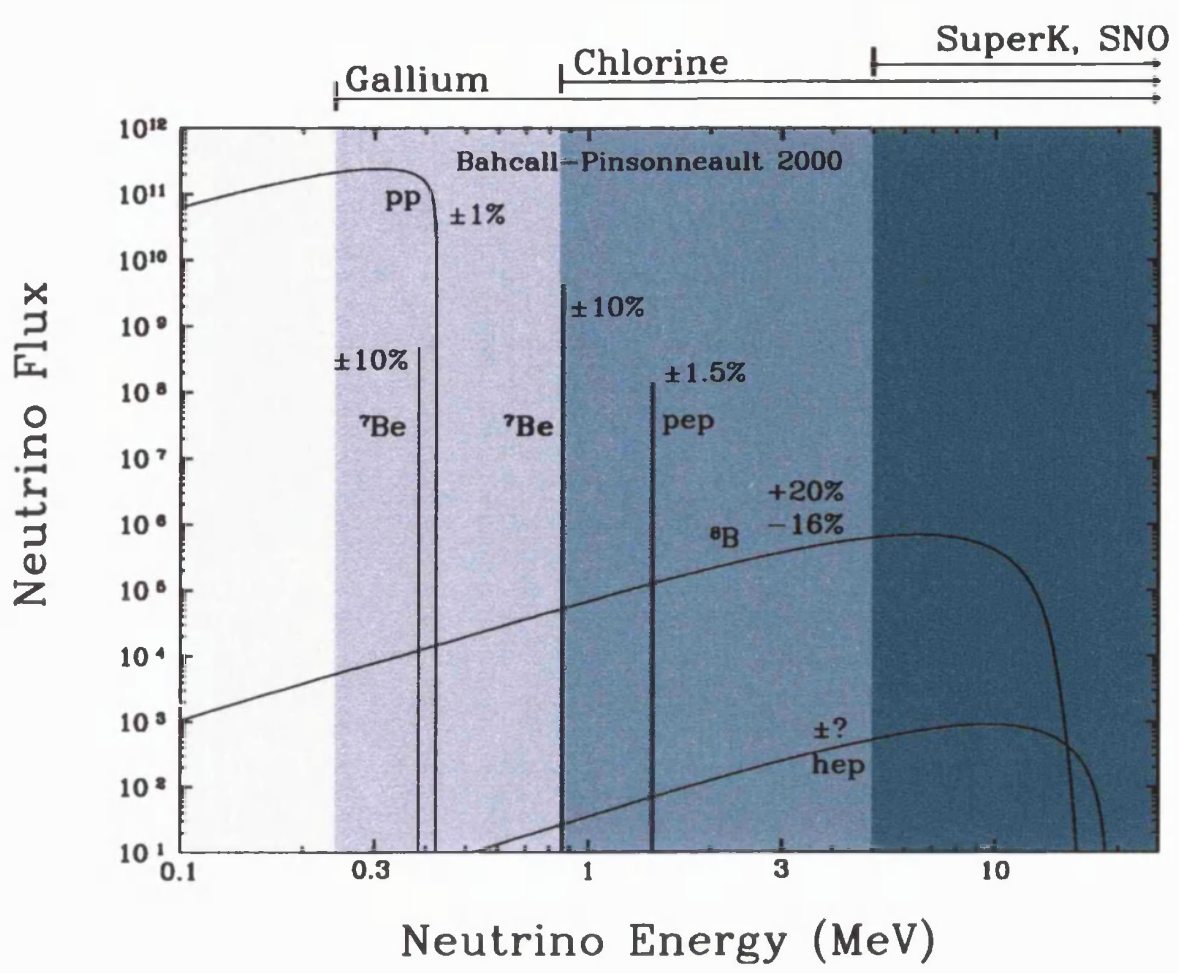

Figure 3.1: The predicted solar neutrino spectrum, the different shaded regions show the energy thresholds for some solar neutrino experiments. From $[22]$

inward facing photomultiplier tubes (PMTs) situated $1000 \mathrm{~m}$ underground in the Kamioka mine in Japan. Neutrinos scatter elastically from electrons in the water, and the recoil electron can then be detected by the ring of Cerenkov light produced as it travels through the water. This method enables the determination of both the direction of the recoil electron, showing whether the neutrino was actually coming from the Sun, and also the energy of the electron. Super-Kamiokande works on the same principle but is larger, containing $50 \mathrm{kT}$ of water and $\sim 11,000$ PMTs.

\subsubsection{SNO}

The Sudbury Neutrino Observatory (SNO) [10][31] is the latest experiment to investigate the solar neutrino problem. Like the Kamiokande detectors it is a water Cerenkov detector, but it differs in that it uses heavy water $\left(D_{2} O\right)$ as opposed to normal water. This allows SNO to distinguish between charged current (CC) electron neutrino events and neutral current (NC) events which all non-sterile neutrinos 
participate in.

In a conventional water Čerenkov detector solar neutrinos, due to their relatively low energy, can only be observed through elastic scattering off an electron. Electron neutrinos can scatter either through charged current or neutral current interactions. However, muon and tau neutrinos can only scatter via the neutral current interactions. As is illustrated in Figure 3.2, charged and neutral current events cannot be distinguished because their final states are the same.

(a)

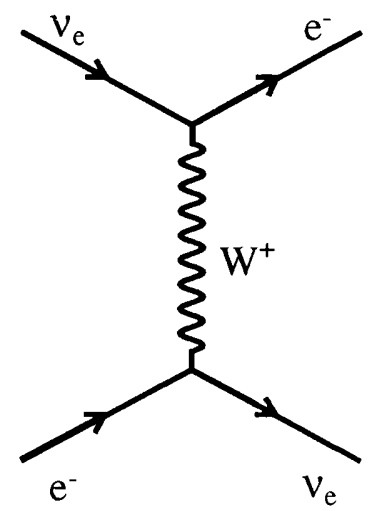

(b)

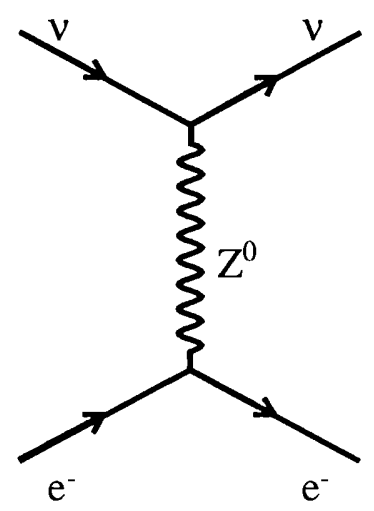

Figure 3.2: Solar neutrino interactions in a conventional water Čerenkov detector. Due to the low energy of the solar neutrinos they can only be observed by elastically scattering off an electron. For electron neutrinos this can occur either through the charged or neutral current channels, the other neutrinos can only scatter via the neutral current interactions. The final states are the same for charged current (a) and neutral current (b) interactions, therefore they are indistinguishable.

The cross-section for a neutrino interacting with a nucleon is much larger than for a neutrino interacting with an electron, as to a first approximation the cross-section scales with the mass of the target. In conventional water Čerenkov detectors these reactions with solar neutrinos are undetectable, in heavy water these interactions are detectable because they can cause the loosely bound deuteron nuclei to break up. This provides a means of distinguishing charged and neutral current events, as illustrated in Figure 3.3, the charged and neutral current events can be expressed 
as

$$
\begin{array}{ll}
C C: & \nu_{e}+{ }^{2} H \rightarrow p+p+e^{-} \\
N C: & \nu+{ }^{2} H \rightarrow p+n+\nu
\end{array}
$$

The interactions split the deuteron providing the energy transferred is greater than $2.2 \mathrm{MeV}$, the deuteron's binding energy. The charged current events are detected through the Čerenkov light produced by the electron, while the neutral current events are detected by the photons produced during neutron capture.

(a)

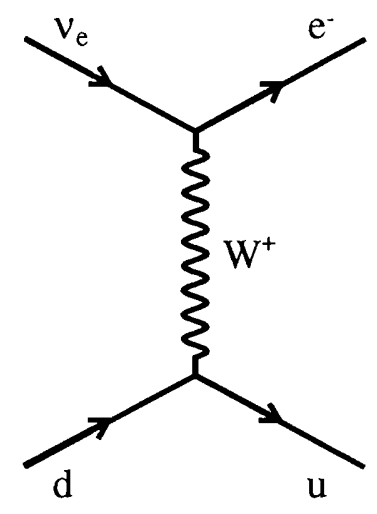

(b)

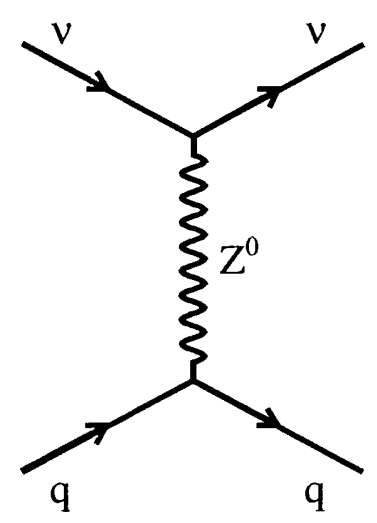

Figure 3.3: Solar neutrino interactions in a heavy water Čerenkov detector. Because the deuteron is only loosely bound, the cross-section for neutrino interactions with the nucleus is greater than for interactions with the electron. This means that charged current (a) and neutral current (b) interactions are distinguishable, as they produce different final states.

There will be three phases of running at SNO, the completed first phase consisted of running with pure heavy water. In the recently finished second phase of running, salt $(\mathrm{NaCl})$ was added to improve the efficiency of neutron capture from $\sim 25 \%$ to $\sim 85 \%$. At present they are beginning the third phase, in which the salt is removed and ${ }^{3} \mathrm{He}$ proportional counters are installed to measure the neutrons directly, with an efficiency of $\sim 45 \%$. This third phase has completely different systematics and so can be used to cross check the previous measurements. 
SNO have published results from the first phase, pure heavy water running, measuring the ${ }^{8} B$ neutrino flux from the Sun. They show that there is direct evidence for a component of the flux which is not electron flavour [10][31]. The measurements of the all flavour flux, through the neutral current interactions, show that the total ${ }^{8} B$ neutrino flux is in close agreement with predictions from the standard solar model.

Recently SNO have published results from the second phase of running with $\mathrm{NaCl}$ added to the heavy water. In this mode of operation the efficiency of neutron capture, and hence neutral current event identification, is increased. The neutral current flux, which is sensitive to all types of neutrino, is measured to be in agreement with calculations from the standard solar model [32].

Figure 3.4 shows how experimental evidence compares to theoretical predictions from the solar model, not including the SNO $\mathrm{NaCl}$ measurement. These experimental results cannot be understood within the current theoretical framework. A global neutrino analysis, using the SNO results and a two-flavour model with matter-enhanced oscillations in the Sun, favours a large mixing angle solution with a best fit of $\Delta m^{2}=7.1_{-0.3}^{+1.0} \times 10^{-5} \mathrm{eV}^{2}$ and $\theta=32.5_{-1.5}^{+1.7}$, [32]. Maximal mixing, with $\sin ^{2} 2 \theta$ equal to unity, is rejected at the $5.1 \sigma$ level.

\subsection{Atmospheric Neutrinos}

Cosmic rays interact with nucleons in the upper atmosphere producing a shower of secondary particles, as illustrated in Figure 3.5. These showers contain many pions which decay via

$$
\begin{aligned}
\pi^{ \pm} \rightarrow & \mu^{ \pm}+\nu_{\mu}\left(\bar{\nu}_{\mu}\right) \\
& \mu^{ \pm} \rightarrow e^{ \pm}+\nu_{e}\left(\bar{\nu}_{\mathrm{e}}\right)+\bar{\nu}_{\mu}\left(\nu_{\mu}\right) .
\end{aligned}
$$

So at the Earth's surface we expect the ratio of $\nu_{\mu}$ to $\nu_{e}$ to be $2: 1$ to the first order.

Atmospheric neutrino detectors can be grouped into two distinct classes: water Čerenkov detectors, these include IMB [34][35], Kamiokande [36] and Super- 
Total Rates: Standard Model vs. Experiment

Bahcall-Pinsonneault 2000

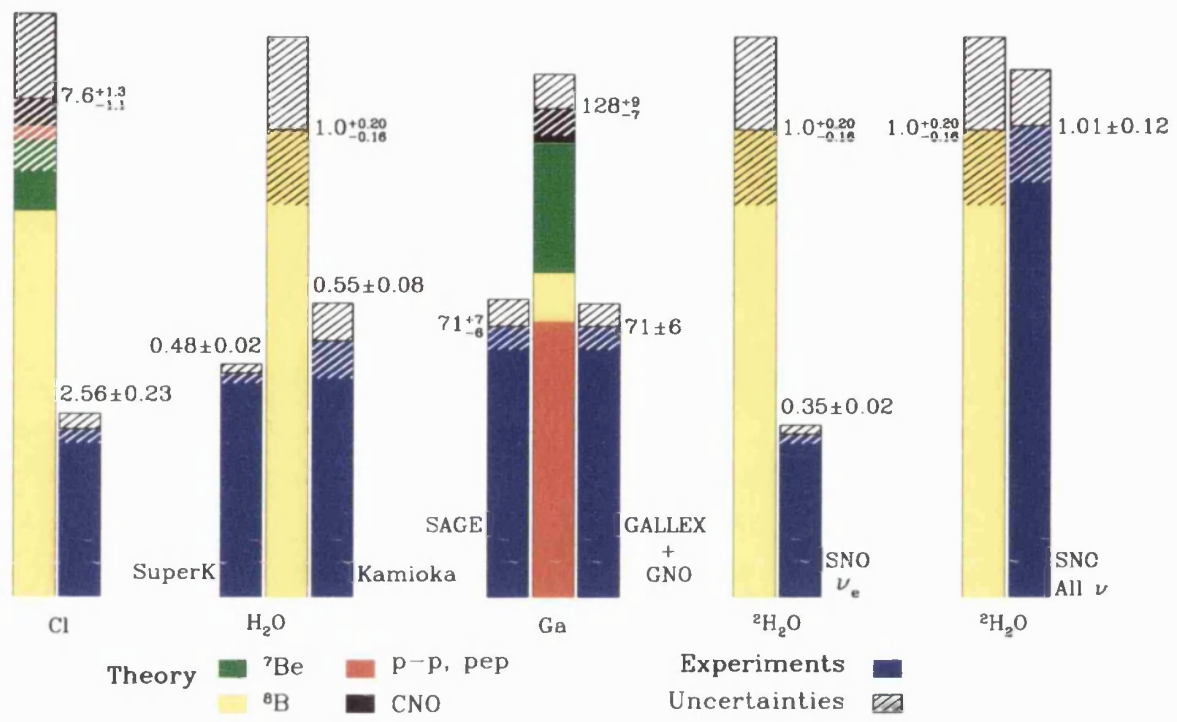

Figure 3.4: Comparison of predicted fluxes, from the solar model, to experimental measurements. Note how the SNO results show an agreement with the predicted flux when all neutrino flavours are considered, but a deficit when looking solely at the electron neutrinos. From $[22]$

Kamiokande [37] and iron sampling calorimeters, like NUSEX [38], Frejus [39] and Soudan $2[40]$.

Experiments studying atmospheric neutrinos usually report their findings in terms of the ratio of ratios,

$$
\mathcal{R} \equiv \frac{\mathcal{R}_{D A T A}}{\mathcal{R}_{M C}}=\frac{\left(N_{\mu} / N_{e}\right)_{D A T A}}{\left(N_{\mu} / N_{e}\right)_{M C}} .
$$

This has the advantage of being independent of experimental parameters, such as geometrical acceptance and energy thresholds, so results from different experiments can be compared directly. Before the Soudan 2 experiment there was a discrepancy between the results of the water Cerenkov and iron calorimeter detectors. This is shown in Figure 3.6. The water Cerenkov experiments measured $\mathcal{R} \sim 0.6$ while the iron calorimeter experiments measured it to be unity. Fears that the discrepancy came from insufficient understanding of the water Cerenkov detectors were laid to rest when Soudan 2, which has higher statistics than the other iron calorimeter 


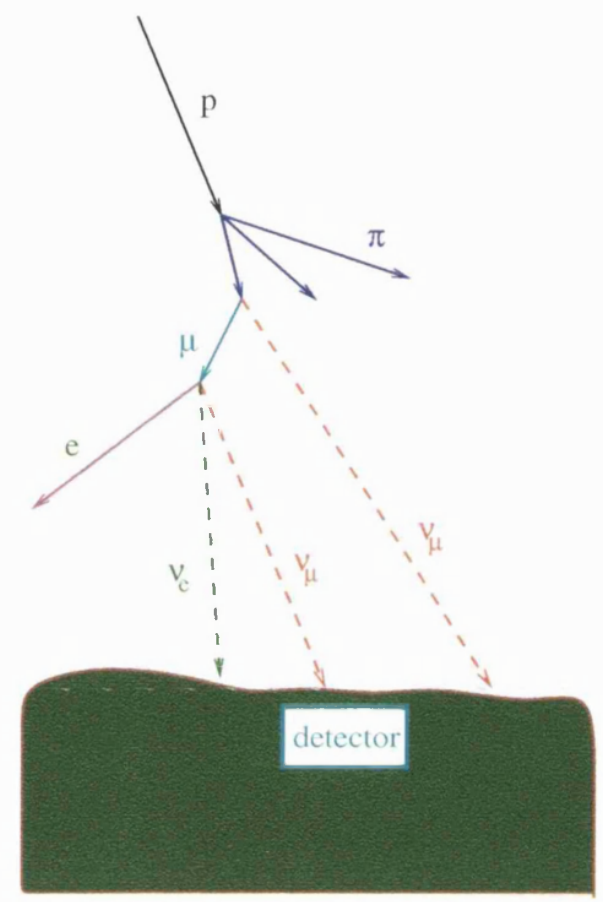

Figure 3.5: Cosmic rays incident in the upper atmosphere interact with nucleons. These interactions produce showers of particles, which decay into muons and muon-neutrinos. In the limit that all the muons decay before reaching the Earth's surface we expect the ratio $\nu_{\mu}: \nu_{e}$ to be $2: 1$. From [33]

experiments, also measured a deficit of $\nu_{\mu}$.

\subsubsection{Super-Kamiokande}

As mentioned in section 3.1, Super-Kamiokande is a $50 \mathrm{kT}$ water Cerenkov detector situated in the Kamioka mine in Japan. It is able to measure the direction and energy of charged particles in the detector by the Cerenkov rings produced in the water. Super-K divides its contained events, those in which the lepton is produced in the detector and does not leave, into sub-GeV and multi-GeV events, where the threshold between the two categories is $1.33 \mathrm{GeV}$. As well as contained events they also analyse partially contained events, muons that are produced in the detector and then leave, and upward through-going muons, muons which are produced in 


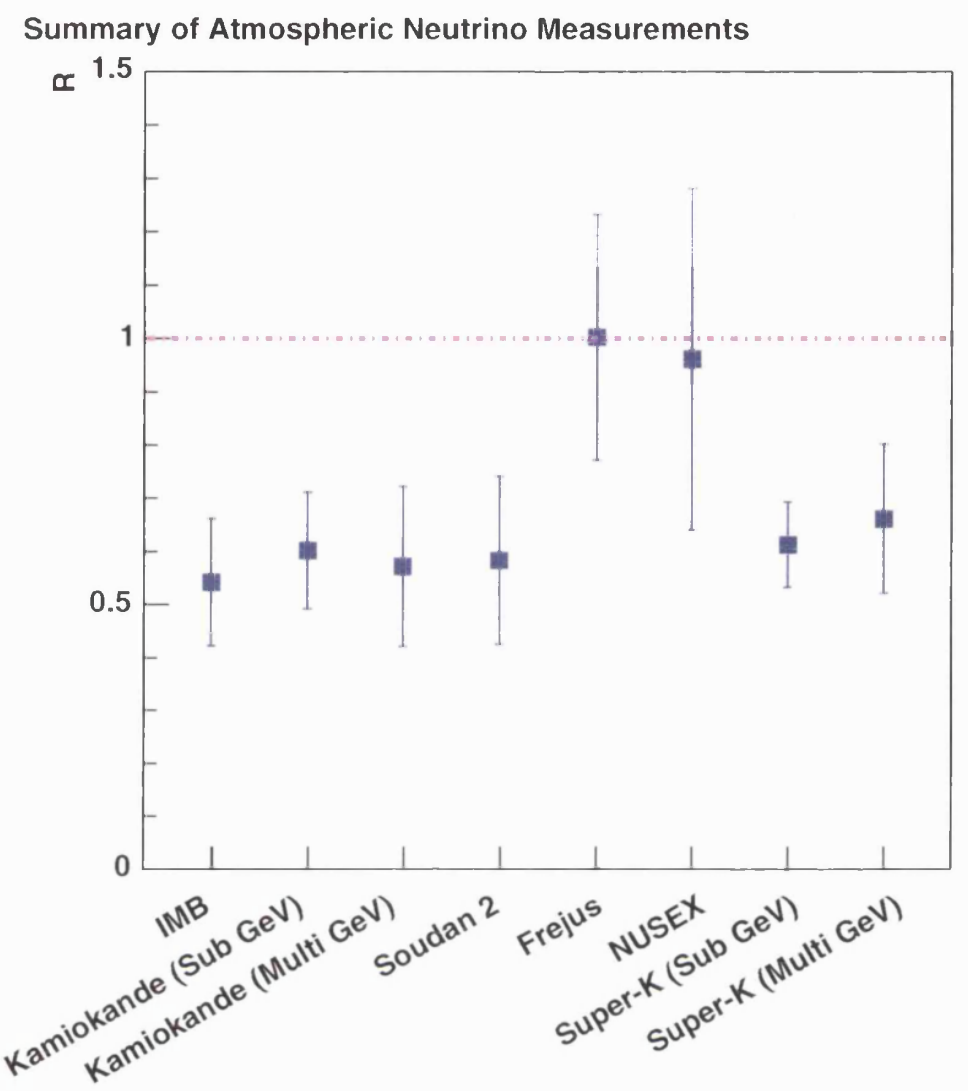

Figure 3.6: Summary of atmospheric neutrino measurements. For each experiment the ratio of ratios is plotted, this value should be one if there are no neutrino oscillations.

the rock under the detector and then pass through the detector.

Super-K is able to test oscillation hypotheses by measuring the zenith angle of the incident neutrino, and hence the distance the neutrino has travelled. These distances range from $10 \mathrm{~km}$ to greater than $10,000 \mathrm{~km}$ as shown in Figure 3.7 . Super-K measured the ratio of ratios, $\mathcal{R}$, to be about $0.6[12]$. At the same time, in 1998, they presented evidence for neutrino mass which was in agreement with the neutrino oscillation hypothesis. They reported a zenith angle effect which differed from the no-oscillation Monte Carlo by $5 \sigma$. They did not report a distortion in the $\nu_{e}$ spectra and so concluded the oscillations must be $\nu_{\mu} \rightarrow \nu_{\tau}$ or $\nu_{\mu} \rightarrow \nu_{\text {sterile. }}$.

With more data, oscillations to $/$ sterile have been excluded at the $99 \%$ confidence limit. If one interprets these results in a two flavour scheme with $\nu_{\mu} \rightarrow \nu_{\tau}$, the best 


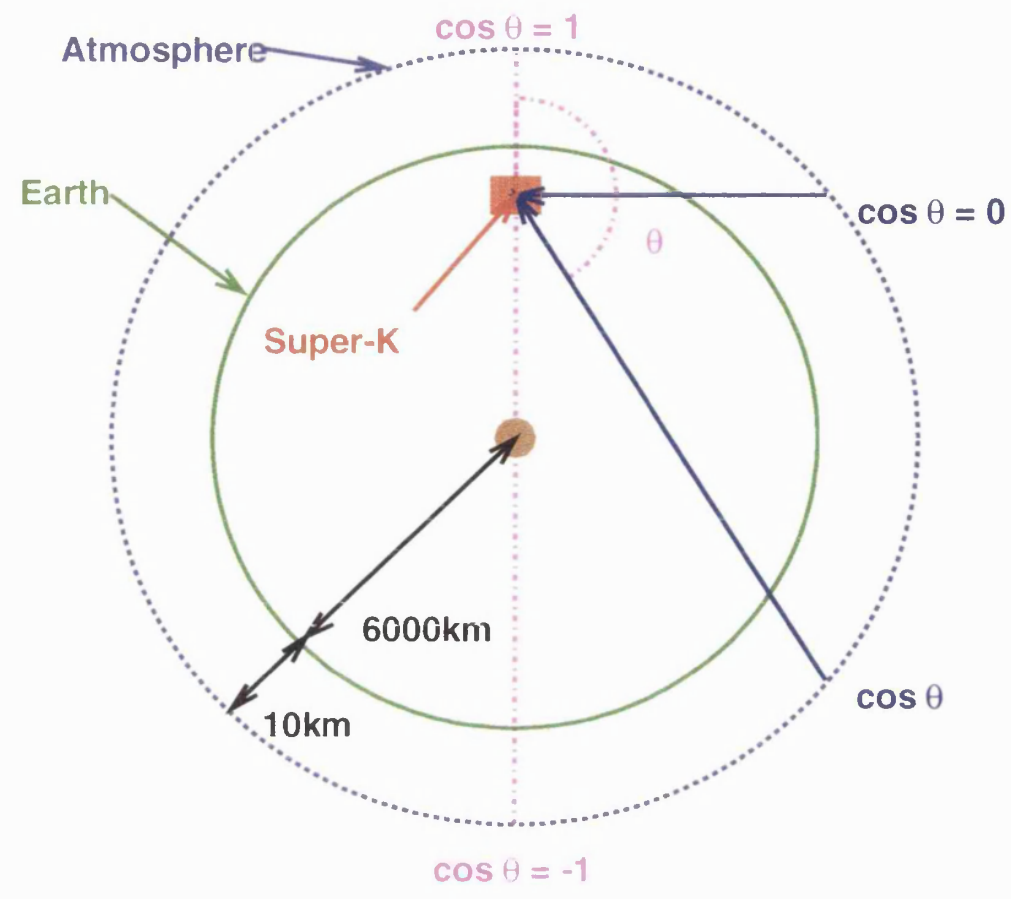

Figure 3.7: Sketch showing how distance travelled by atmospheric neutrinos varies with zenith angle. Super-Kamiokande uses this dependence to test oscillation hypotheses.

fit values are for maximal mixing, $\sin ^{2} 2 \theta=1$, and $\Delta m^{2}=2.5 \times 10^{-3} \mathrm{eV}^{2}[41]$.

\subsection{Reactor Neutrinos}

Nuclear reactors produce $\bar{\nu}_{\mathrm{e}}$ during the fission of heavy nuclei like ${ }^{235} \mathrm{U}$ and ${ }^{239} \mathrm{Pu}$. It is possible to detect these anti-neutrinos via the inverse $\beta$-decay reaction $\bar{\nu}_{\mathrm{e}}+p \rightarrow$ $e^{+}+n$. Until very recently no experiments had detected any evidence of neutrino oscillations from reactor neutrinos. Before 2002, the most sensitive experiment was the CHOOZ experiment [42], which was located $\sim 1 \mathrm{~km}$ away from the reactor core of the Chooz power station. The experiment finished taking data in 1998, and following a full analysis they found no evidence of spectral distortion. This has excluded a large area of oscillation parameter space, and indicated that $\nu_{\mu} \rightarrow \nu_{e}$ could not be the cause of the atmospheric neutrino problem. This result agrees with the Super- 
Kamiokande interpretation that the cause of the problem is $\nu_{\mu}$ disappearance as opposed to $\nu_{e}$ appearance.

\subsubsection{KamLAND}

The Kamioka Liquid scintillator Anti-Neutrino Detector (KamLAND) experiment is the first experiment to report evidence for reactor anti-neutrino disappearance [16]. The experiment differs from previous reactor based experiments in the extended length of its baseline, which is an average of $180 \mathrm{~km}$. This extended baseline allows KamLAND to probe smaller values of $\Delta m^{2}$ than previous reactor based experiments. An average value of the baseline is quoted as the detector is near a large number of nuclear reactors. They calculate that $79 \%$ of their neutrino flux comes from 26 reactors that are within the distance range $138-214 \mathrm{~km}$.

The detector consists of $1 \mathrm{kT}$ of ultra pure liquid scintillator contained in a $13 \mathrm{~m}$ diameter spherical balloon. An array of 1,879 inwards facing PMTs is mounted outside the balloon. This inner detector is surrounded by a water Cerenkov detector which is used for tagging cosmic ray muons. The $\bar{\nu}_{\mathrm{e}}$ are detected by the inverse $\beta$ decay reaction $\bar{\nu}_{\mathrm{e}}+p \rightarrow e^{+}+n$ in the liquid scintillator, this method is sensitive to $\bar{\nu}_{\mathrm{e}}$ with energy above $1.8 \mathrm{MeV}$. Requiring a coincidence between the $e^{+}$and the delayed $\gamma$-ray from neutron capture on a proton enables is a powerful tool for separating these events from the background.

After calculating the combined flux at the detector from all the reactors, KamLAND claim that the ratio of observed events over expected events is $\sim 0.6$. This shows reactor $\bar{\nu}_{\mathrm{e}}$ disappearance at the $99.95 \%$ confidence limit. The best fit to the $\bar{\nu}_{\mathrm{e}} \rightarrow \overline{\nu_{x}}$ oscillation parameters is for the maximal mixing with $\Delta m^{2}=6.9 \times 10^{-5} \mathrm{eV}^{2}$. Assuming that CPT invariance ${ }^{1}$ holds this result is in agreement with the large mixing angle solution from solar neutrino experiments.

\footnotetext{
${ }^{1} \mathrm{CPT}$ invariance is the scemingly fundamental symmetry achieved from the combination of charge conjugation $(\mathrm{C})$, parity $(\mathrm{P})$ and time reversal $(\mathrm{T})$. If CPT invariance holds then neutrino oscillation parameters should be identical to those of anti-neutrinos
} 


\subsection{Accelerator Neutrinos}

Accelerator based neutrino experiments produce neutrinos by firing beams of high energy particles at targets, producing large numbers of secondary pions and kaons which can be steered by magnets before decaying into muons and muon neutrinos. The advantage of an accelerator based experiment is that one has greater knowledge, and control, over the energy and flavour content of the neutrinos produced. This should allow better determination of the oscillation parameters.

\subsubsection{Short Baseline Experiments}

In a short baseline experiment the neutrino detector is situated approximately $1 \mathrm{~km}$ or less from the source, which produces neutrinos with energies ranging from $10^{-2} \mathrm{MeV}$ to $\sim 10 \mathrm{GeV}$. This results in a sensitivity to oscillations down to $\Delta m^{2} \sim$ $0.1 \mathrm{eV}^{2}$.

The experiments CHORUS [43] and NOMAD [44], both CERN based, have looked for $\nu_{\tau}$ appearance. Neither experiment found any evidence for oscillations, thus excluding $\Delta m^{2}>1 \mathrm{eV}^{2}$ for $\nu_{\mu} \rightarrow \nu_{\tau}$.

The only short baseline experiment to report a positive signal thus far is the Liquid Scintillator Neutrino Detector (LSND) [45], an electron neutrino appearance experiment at Los Alamos. They produce a beam of mainly $\pi^{+}$, with a small fraction of $\pi^{-}$, from a proton beam incident on a target. They then used two classes of neutrino: those from pion decay in flight (DIF), via $\pi^{+} \rightarrow \mu^{+}+\nu_{\mu}$, and also those from muon decay at rest (DAR), via $\mu^{+} \rightarrow e^{+}+\nu_{e}+\bar{\nu}_{\mu}$.

The $\bar{\nu}_{\mathrm{e}}$ are detected via $\bar{\nu}_{\mathrm{e}}+p \rightarrow e^{+}+n$, where the signal comes from the positron and a delayed $2.2 \mathrm{MeV}$ photon from the neutron capture. Whereas $\nu_{e}$ are detected via $\nu_{e}+C \rightarrow e^{-}+X$. They reported an excess of $\bar{\nu}_{\mathrm{e}}$ in both the DAR and DIF sources, this corresponds to $\bar{\nu}_{\mu} \rightarrow \bar{\nu}_{\mathrm{e}}$ oscillations with $\Delta m^{2} \sim 1 \mathrm{eV}^{2}$ and $\sin ^{2} 2 \theta \sim 10^{-2}$.

Other experiments have also looked for $\nu_{e}$ appearance, one such experiment is KARMEN [46] which was located at the Rutherford Laboratory in the UK. KAR- 
MEN is a liquid scintillator detector with a baseline of $17.5 \mathrm{~m}$, about half the LSND baseline. It has measured no evidence of neutrino oscillations, thus excluding a large part of the LSND preferred parameter space.

The BooNE project, of which MiniBooNE [47] is the first stage, attempts to confirm or refute the LSND findings. It started taking data in 2002 but has yet to report any findings. Should oscillations be present in the region suggested by LSND then after one years running MiniBooNE will observe a 8-10 $\sigma$ signal. If the LSND result proves to be anomalous, then after two years running all of the LSND parameter space can be excluded at the $90 \%$ confidence limit.

\subsubsection{Long Baseline Experiments}

As their name suggests, long baseline experiments differ from the short baseline experiments in the distance of the detector from the source of neutrinos, typically hundreds of kilometres.

The first, and so far only active, long baseline experiment was built between KEK and the Kamioka mine in Japan, and is called K2K [48]. A 98\% pure beam of $\nu_{\mu}$ from the KEK accelerator facility is aimed towards the Super-Kamiokande detector in the Kamioka mine, some $250 \mathrm{~km}$ away. There is a near detector on site at KEK. The experiment looks for $\nu_{\mu}$ disappearance between the near detector and the Super-K detector. The average energy of the neutrinos is $1.3 \mathrm{GeV}$, so $\nu_{\tau}$ appearance is not possible.

In 2002, K2K observed indications of neutrino oscillations [49]. They observed a deficit of $\nu_{\mu}$ at the far detector and a distortion of the energy spectrum, which is better matched to the theoretical spectrum with oscillations than without. The probability, under the no-oscillation hypothesis, of obtaining data as "oscillation like" or more so as that obtained is less than $1 \%$. The best fit values for the $\nu_{\mu} \rightarrow \nu_{\tau}$ oscillation parameters are $\sin ^{2} 2 \theta=1$ and $\Delta m^{2}=2.8 \times 10^{-3} \mathrm{eV}^{2}$. The $90 \%$ C.L contour crosses the $\sin ^{2} 2 \theta=1$ line at 1.5 and $3.9 \times 10^{-3} \mathrm{eV}^{2}$. These values are consistent with the best-fit values from Super-K atmospheric neutrino measurements. 
Two other long baseline experiments are currently in construction, and plan to start data taking within the next few years. The CERN Neutrinos to Gran Sasso (CNGS) [50] experiment, intends to fire a beam of mainly $\nu_{\mu}$ neutrinos from CERN, $732 \mathrm{~km}$ through the ground, to the Gran Sasso underground facility. Unlike the K2K experiment the CERN beam will be of a high enough energy, $17 \mathrm{GeV}$ average, to look for $\nu_{\tau}$ appearance. At Gran Sasso there will be two large detectors, the OPERA lead/emulsion based detector and the ICARUS liquid argon TPC. The other long baseline neutrino oscillation experiment set to start in the next few years is the NuMI-MINOS project, which will be explained in detail in section 4 .

\subsection{Summary}

If one assumes that all the above experimental results are a direct consequence of neutrino oscillations, they can be summarized as:

- Results from both solar neutrino experiments, in particular SNO, and also from KamLAND's reactor neutrino measurements suggesting $\nu_{e} \rightarrow \nu_{x}$ oscillations with the large mixing angle favoured and best fits to the oscillations of $\Delta m^{2}=7.1_{-0.3}^{+1.0} \times 10^{-5} \mathrm{eV}^{2}$ and $\theta=32.5_{-1.5}^{+1.7}$

- Atmospheric neutrino measurements are backed up with the first long baseline results from $\mathrm{K} 2 \mathrm{~K}$ in suggesting, $\nu_{\mu} \rightarrow \nu_{\tau}$ oscillations with maximal mixing and $\Delta m^{2} \sim 10^{-3} \mathrm{eV}^{2}$

- LSND are alone, so far, in showing evidence of $\nu_{\mu} \rightarrow \nu_{e}$ oscillations with $\sin ^{2} 2 \theta=10^{-2}$ and $\Delta m^{2} \sim 1 \mathrm{eV}^{2}$.

Alternatively, this information can be summarized visually in oscillation parameter space. Figure 3.8 shows the $\nu_{\mu} \rightarrow \nu_{\tau}$ parameter space and Figure 3.9 is for $\nu_{\mu} \rightarrow \nu_{e}$ oscillations, in each plot the relevant oscillation is assumed to dominate.

In order to explain all the data we need three different $\Delta m^{2}$. However since $\Delta m_{12}^{2}+\Delta m_{13}^{2}+\Delta m_{23}^{2}=0$, and we have $\Delta m_{S O L}^{2} \ll \Delta m_{A T M}^{2} \ll \Delta m_{L S N D}^{2}$, it is not possible to accommodate all these results within a three generation neutrino 


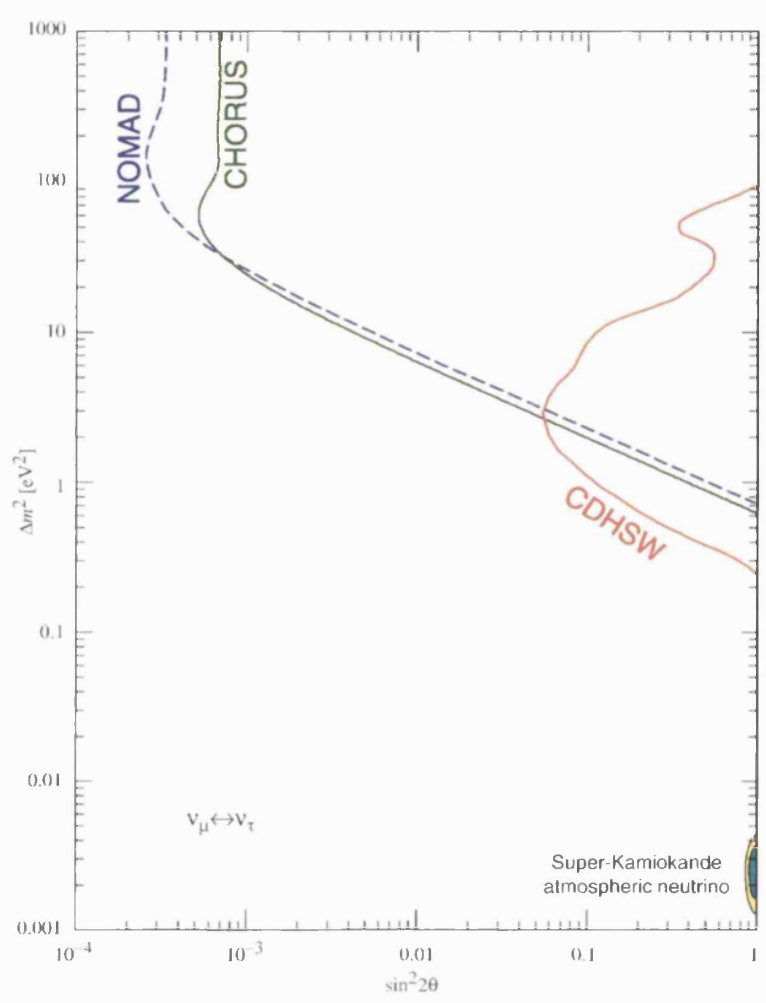

Figure 3.8: Allowed and excluded regions of parameter space for $\nu_{\mu} \leftrightarrow \nu_{\tau}$ oscillations. The excluded regions from the CERN short baseline $\nu_{\tau}$ appearance experiments are shown, along with the allowed region from Super-Kamiokande. From [51]

mixing model. In addition to this we know, from LEP that there are only 3 light neutrinos [5] [6][7][8], with mass less than half $M_{Z}$, meaning a fourth neutrino would have to be sterile.

At present the situation regarding neutrino oscillations is unclear, and therefore there is need for more experimental data. This is where MINOS, and other new experiments enter the picture. MINOS will be able to definitively measure whether the atmospheric neutrino results are due to $\nu_{\mu} \rightarrow \nu_{\text {sterile }}$ or $\nu_{\mu} \rightarrow \nu_{\tau}$, and measure the oscillation parameters, $\Delta m^{2}$ and $\sin ^{2} 2 \theta$, to $10 \%$. The BooNE project will attempt to confirm or exclude the LSND result, while SNO and KamLAND will continue investigating the $\nu_{e}$ and $\bar{\nu}_{\mathrm{e}}$ oscillation parameters. Over the course of the next few years, the physics of the neutrino sector should become much clearer. 


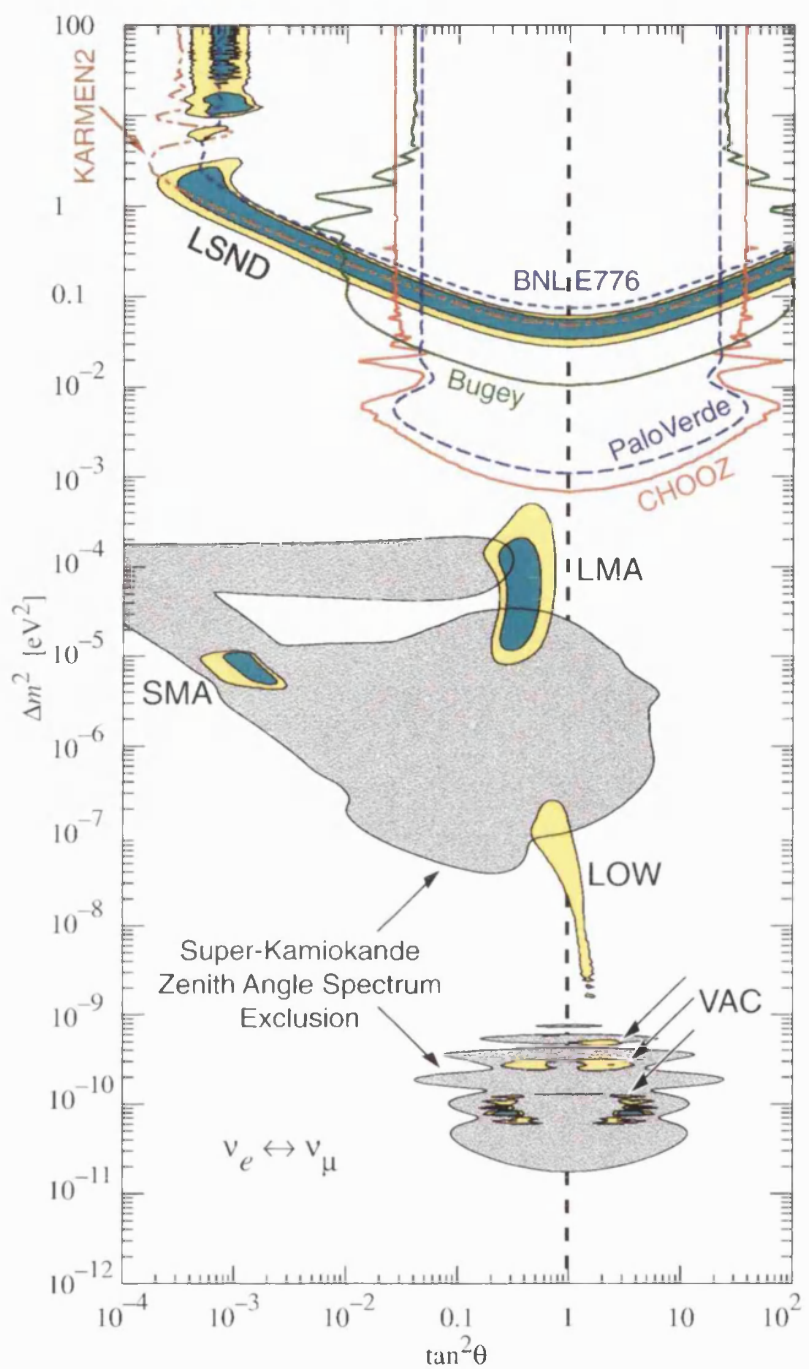

Figure 3.9: Allowed and excluded regions of parameter space for $\nu_{\mu} \leftrightarrow \nu_{e}$ oscillations. The excluded regions from KARMEN and BNL are from $\nu_{e}$ appearance, as is the preferred region from LSND. The CHOOZ, Bugey and Palo Verde excluded regions are all from $\bar{\nu}_{\mathrm{e}}$ disappearance. The preferred solar regions are labelled LMA (large mixing angle), SMA (small mixing angle), LOW (low $\Delta m^{2}$ ) and VAC (the vacuum or "Just-so" solution). These regions are from $\nu_{e}$ disappearance at Homestake, SAGE, GALLEX, GNO and Super-Kamiokande. Also shown is the excluded region from the zenith angle spectrum at Super-Kamiokande. This does not include the latest results from SNO, these only leave the LMA solution. From [51] 


\section{Chapter 4}

\section{The NuMI-MINOS Experiment}

The NuMI-MINOS experiment is a long baseline neutrino oscillation experiment. A beam of $98 \%$ muon neutrinos is created at Fermilab and fired through the ground towards the Soudan mine in northern Minnesota, see Figure 4.1. The Near Detector, which is on site at Fermilab, makes a measurement of the beam to compare with that made by the Far Detector $735 \mathrm{~km}$ away in the mine in Minnesota. Through this comparison neutrino oscillations can be inferred from differences in the neutrino spectra. This section will describe the three main components of the experiment:

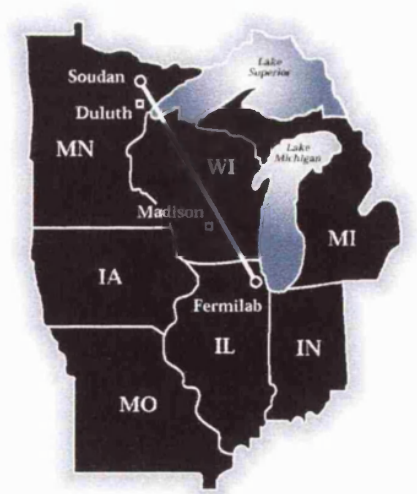

Figure 4.1: The path of the NuMI neutrino beam.

the NuMI beam, the Near Detector and the Far Detector. The physics capabilities of the experiment will also be discussed. For more detailed information one should consult the technical design reports of the NuMI facility [52] and also of the MINOS 
experiment $[53]$.

\subsection{The NuMI Beam}

The Neutrinos at the Main Injector (NuMI) beam, is a neutrino beam created at the Main Injector facility in Fermilab. When it turns on, it will be the most intense source of neutrinos on the Earth. The beam will consist largely of muon neutrinos formed from the decay of $\pi$ 's and K's. The $\pi$ 's and $K$ 's are created when a $120 \mathrm{GeV}$ beam of protons from the Main Injector is focused at a carbon target. When the beam begins operation in 2004, the Main Injector is expected to deliver $3 \times 10^{13}$ protons per $10 \mu \mathrm{s}$ spill.

A schematic of the beam line is shown in Figure 4.2. There are two parabolic magnetic horns after the target which are used to focus the charged hadrons produced. After the focusing the hadrons pass through a $675 \mathrm{~m}$ evacuated decay pipe. in which they will decay producing muons and muon neutrinos. At the end of the decay pipe is a water-cooled hadron absorber to absorb any remaining hadrons and after this there is a further $240 \mathrm{~m}$ of rock, before the Near Detector, to absorb the muons.

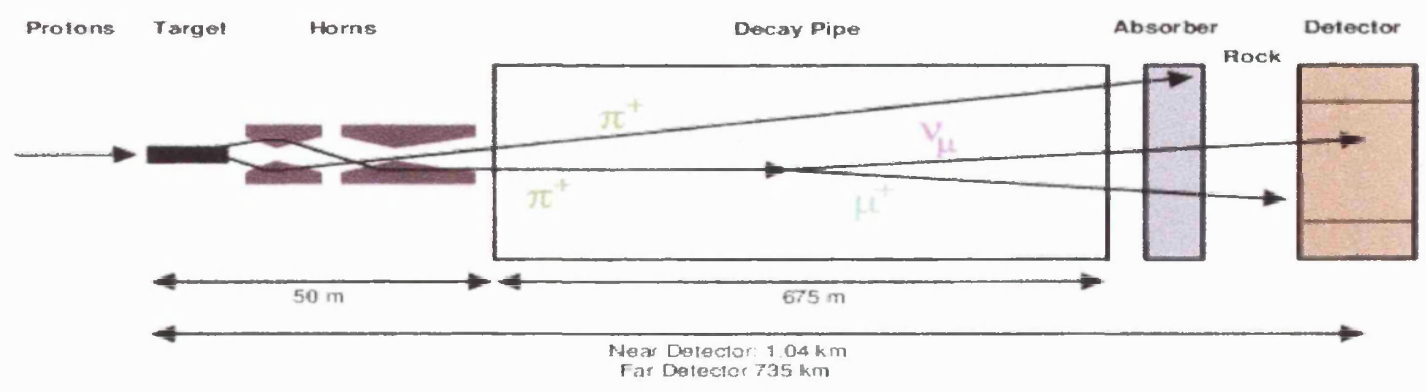

Figure 4.2: The NuMI beamline. Protons from the Main Injector are incident on the target producing secondary hadrons. The charged hadrons are focused by the magnetic horns before decaying into muons and muon neutrinos in the decay pipe. The absorber and rock remove the muons and any undecayed hadrons, leaving a beam of neutrinos.

The two parabolic horns and the target are movable. This allows a certain 
degree of flexibility in the energy spectrum of neutrinos produced. There are three standard configuration modes; the so called low, medium and high energy beams. The different horn arrangements and unoscillated energy spectra at the Far Detector for the three configurations are shown in Figure 4.3.
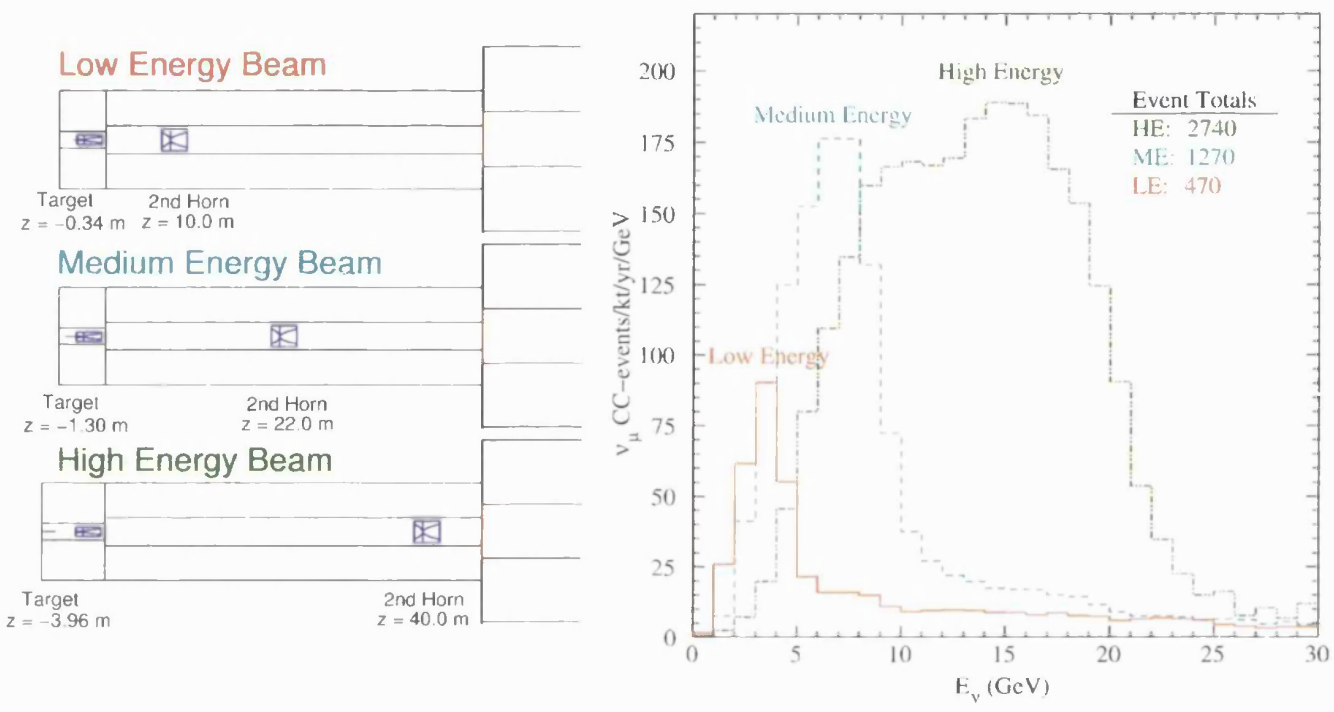

Figure 4.3: The three configurations of the NuMI beam. On the left is the target and horn arrangement for the low, medium and high energy beams. On the right is the $\nu_{\mu} \mathrm{CC}$ Far Detector energy spectra, in the case where there are no oscillations, for each of the beam configurations.

Initially the beam will be configured with the low energy option. This is to maximize the experiments sensitivity to oscillations in the region of parameter space suggested by other experiments, most notably Super-Kamiokande.

As the beam travels from Fermilab to Soudan it diverges to approximately one kilometre in diameter. This divergence means that the energy spectrum of neutrinos at the Far Detector is different to that at the Near Detector, because the Far Detector samples only the central region of the beam. One of the largest uncertainties in the neutrino flux and energy spectrum at the Far Detector, as extrapolated from Near Detector measurements, comes from divergence of the decay kinematics of the charged hadrons in the NuMI beamline. The Main Injector Particle Production 
(MIPP) [54] is a hadron production experiment at Fermilab. It has been designed, in part, to measure the particles produced, and their kinematics, when a beam of protons are incident on the NuMI carbon target. The results from MIPP will help to minimize uncertainties in the flux and energy spectrum of neutrinos at the Far Detector.

\subsection{The MINOS Detectors}

There are three MINOS detectors which have been designed to be as similar as possible to each other. In order of size, largest first, these are the Far Detector, the Near Detector and finally the Calibration Detector. The Calibration Detector is the focus of Chapter 6 , and so will not be discussed explicitly in this section. As the detectors are very similar in design, it is worth discussing the main features of the design before detailing the differences between the detectors.

\subsubsection{Generic MINOS Detector}

The MINOS detectors are fine grained steel-scintillator tracking calorimeters. They feature the classic 'sandwich' design, with an absorber layer of steel followed by an active layer of plastic scintillator. The steel is $2.54 \mathrm{~cm}$, one inch, thick and the scintillator is $1 \mathrm{~cm}$ thick. Between the steel and the scintillator is an air gap, for mechanical construction reasons, making each steel-air-scintillator layer of the sandwich $6 \mathrm{~cm}$ thick.

The scintillator is made of polystyrene, the fluor PP0 (1\%) and the wavelength shifter POPOP $(0.030 \%)$. Each plane of scintillator is constructed from individual strips $4.1 \mathrm{~cm}$ wide, with up to 192 strips in a plane. The orientation of these strips changes by $90^{\circ}$ in successive planes, providing 3-dimensional tracking capabilities. The detector has a resolution of $\sim 23 \% / \sqrt{E}$ for electromagnetic showers and $\sim 55 \% / \sqrt{E}$ for hadronic showers.

The scintillator is read out by $1.2 \mathrm{~mm}$ diameter Kurray wavelength shifting fibre lying in a groove along the length of the scintillator strip, see Figure 4.4. Between 


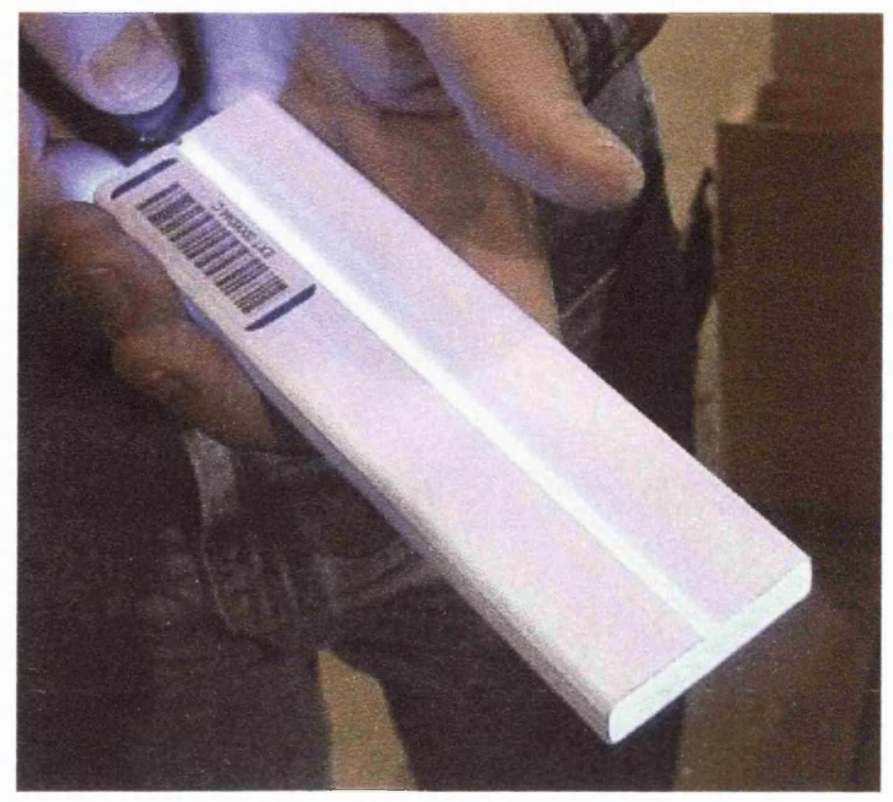

Figure 4.4: A short piece of MINOS scintillator illuminated by a blue LED. The reflective coating around the scintillator and the groove for the wavelength shifting fibre are clearly visible.

16 and 28 strips are enclosed in a light tight aluminium case, forming a scintillator module. At the end of these modules the wavelength shifting fibres run to a connector, from where clear fibres transport the light to boxes containing Hamamatsu multi-anode photomultiplier tubes.

The Near and Far Detectors are magnetized by a current carrying coil running through the centre of the detector. This coil provides a toroidal magnetic field in the steel which ranges from approximately 1 to $1.5 \mathrm{~T}$, within the fiducial volume. This field allows determination of the momentum and charge of muons from track curvature. However, the resolution obtained is limited by multiple scattering in the steel, for example a $10 \mathrm{GeV}$ muon can only be measured to $14 \%$. Low energy muons which stop in the detector can be better measured by range, this gives a resolution of approximately $6 \%[55]$. For the low energy beam, which MINOS will begin operation with, over $50 \%$ of muons produced in the Far Detector stop [56]. 


\subsubsection{The Far Detector}

The recently completed Far Detector is situated almost $700 \mathrm{~m}$ underground in the Soudan mine, in northern Minnesota. The engineering required for assembling the $5.4 \mathrm{kT}$ detector is made more difficult by the fact that everything required for the detector and its construction must travel underground in the $2 \times 1 \times 8 \mathrm{~m}$ lift cage. This means that each of the 8 metre diameter octagonal steel and scintillator planes that make up the detector was hauled underground in 8 sections and welded together at the bottom.

The detector, which is the largest of the MINOS detectors, consists of 484 instrumented planes of scintillator, with two extra steel planes. The detector is split into two super-modules each with its own coil and magnetic field. This has meant that cosmic muons and atmospheric neutrino data have been collected in the magnetized first super-module, whilst the second was under construction. See Figure 4.5 for a picture of the completed detector.

Each of the planes of scintillator is split into 192 strips which are arranged in 8 modules. The strips are read out, at each end, with Hamamatsu M16 multianode photomultiplier tubes (PMTs). Due to the low rates, on the order of one beam neutrino event per hour and one cosmic ray muon per second, and small transverse size of events in the Far Detector it has been possible to multiplex the readout, such that 8 strips from a plane are readout by each of the pixels on a PMT. Strips connected to the same pixel are separated by an average of $1 \mathrm{~m}$ in the plane. Demuxing is possible due to the different permutations of multiplexing used on either side of the detector. Each plane in the detector is read out by a pair of Mux boxes, one at either end, containing three M16 PMTs in each box. The factor of 8 fewer PMTs and electronics channels needed due to the multiplexing, results in a considerable cost saving.

The phototubes are read out using a version of the Viking chip made by IDE AS of Norway, known as VA chips. This chip samples and holds the anode signals of the PMTs before they are digitized in turn by the ADC. The front end electronics are readout by the DAQ via VME crates and single board readout processors, the 


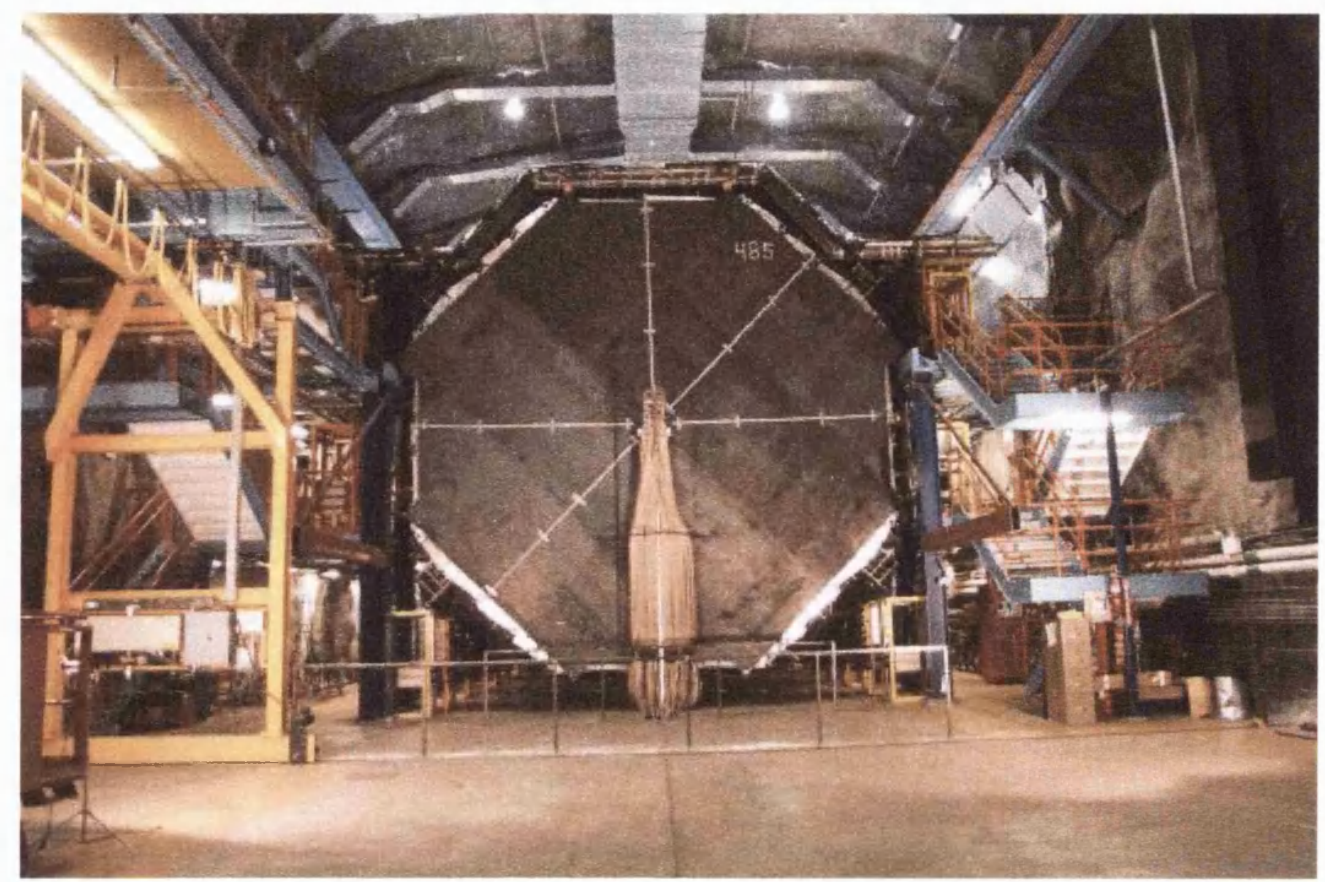

Figure 4.5: A photo showing the completed Far Detector, which is situated in the Soudan mine. The detector consists of 486 planes of steel, planes 0 to 485 , and 484 planes of scintillator. Coming from the centre of the last plane, the coil and the coil cooling system are clearly visible. The coil provides the magnetic field which magnetizes the steel planes. Also visible in the picture are the black optical readout cables which run from the scintillator planes to the Mux boxes, containing the PMTs.

RIO processors made by CES of Switzerland.

In Figure 4.5 the top and bottom levels on either side house the Mux boxes. On the middle level there are the VME readout crates, the pulser boxes (used for calibration purposes, see Chapter 5) and the Lecroy 1440 high voltage supplies.

Since the first super-module was completed and magnetized the detector has been taking atmospheric neutrino and cosmic ray data. To improve the detector's atmospheric neutrino measurement capability a veto shield has been constructed around the top and side of the detector, see Figure 4.6. The main purpose of this shield is to tag cosmic muons entering the detector through the steel or air gaps, and appearing like neutrino interactions within the detector. It greatly enhances the ability to correctly identify contained or partially contained neutrino events in 
the detector.

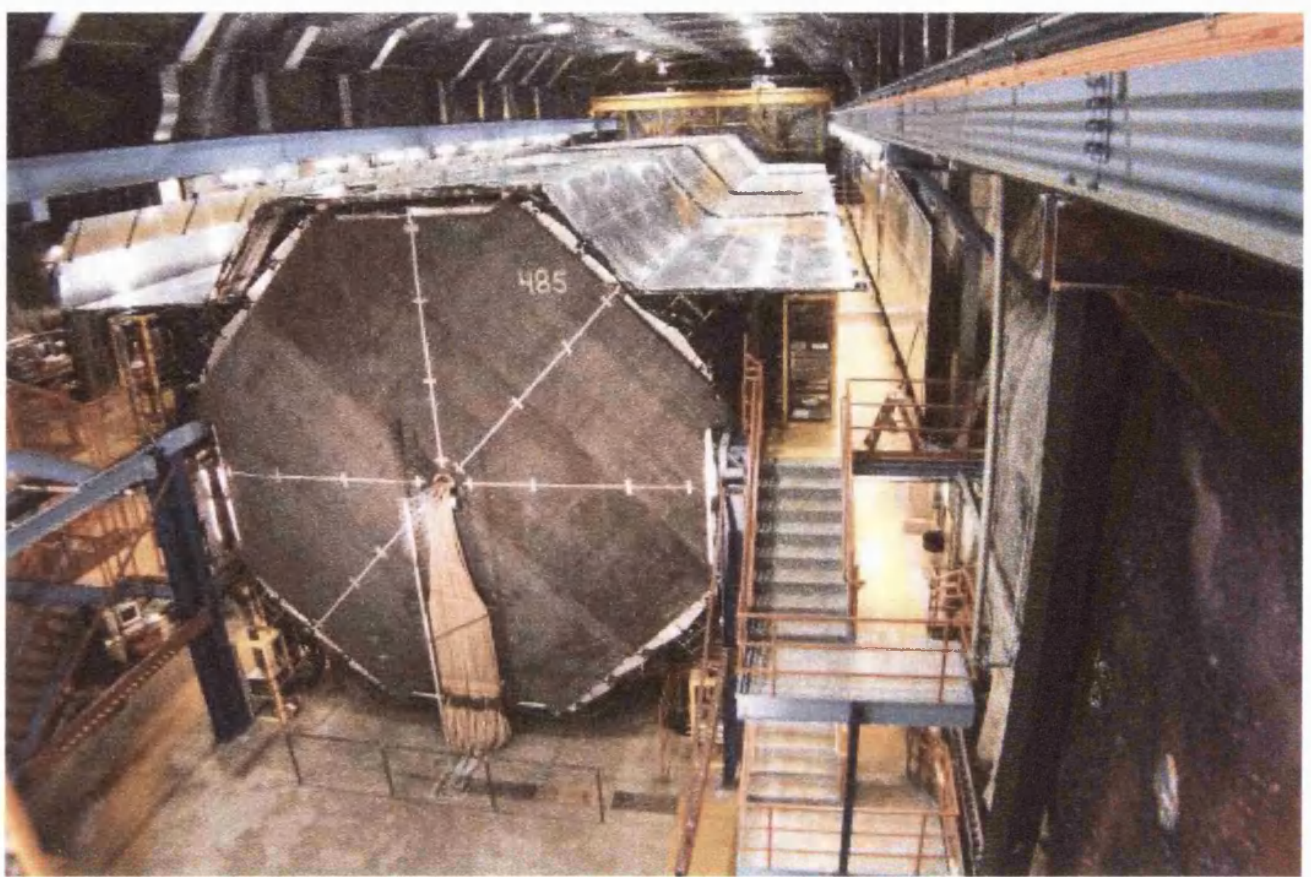

Figure 4.6: The veto shield at the Far Detector. The veto shield was installed as the Far Detector started to collect atmospheric neutrino data. The shield provides a method for tagging cosmic ray muons that enter the detector through the steel or air gaps. These muons would otherwise look similar to neutrino interactions in the detector. The shield consists of layers of scintillator across the top of the detector and down the walls of the cavern, giving coverage over the majority of angles and positions which cosmic ray muons enter the detector.

\subsubsection{The Near Detector}

The Near Detector is located on site at Fermilab and serves as a zero reference point to the measurements made at the Far Detector. It was designed to be as similar as possible to the Far Detector, whilst attempting to reduce the amount of steel, scintillator, instrumentation and hence cost, without compromising the physics capabilities. The differences in design between the two detectors stem from the fact that the beam is $\sim 1 \mathrm{~km}$ in diameter at the Far Detector and the central region is $50 \mathrm{~cm}$ in diameter at the Near Detector, resulting in a much higher flux at 
the Near Detector. The detector is in the shape of a 'squashed' octagon, $3.8 \mathrm{~m}$ high and $4.8 \mathrm{~m}$ wide, as can be seen in Figure 4.7. Both the coil hole and the neutrino beam spot are offset $50 \mathrm{~cm}$ from the centre of the plane, to achieve a similar magnetic field to that of the fiducial region of the Far Detector and to avoid event loss down the coil hole. The detector weighs in at 980 tons, and consists of 282 planes of steel.
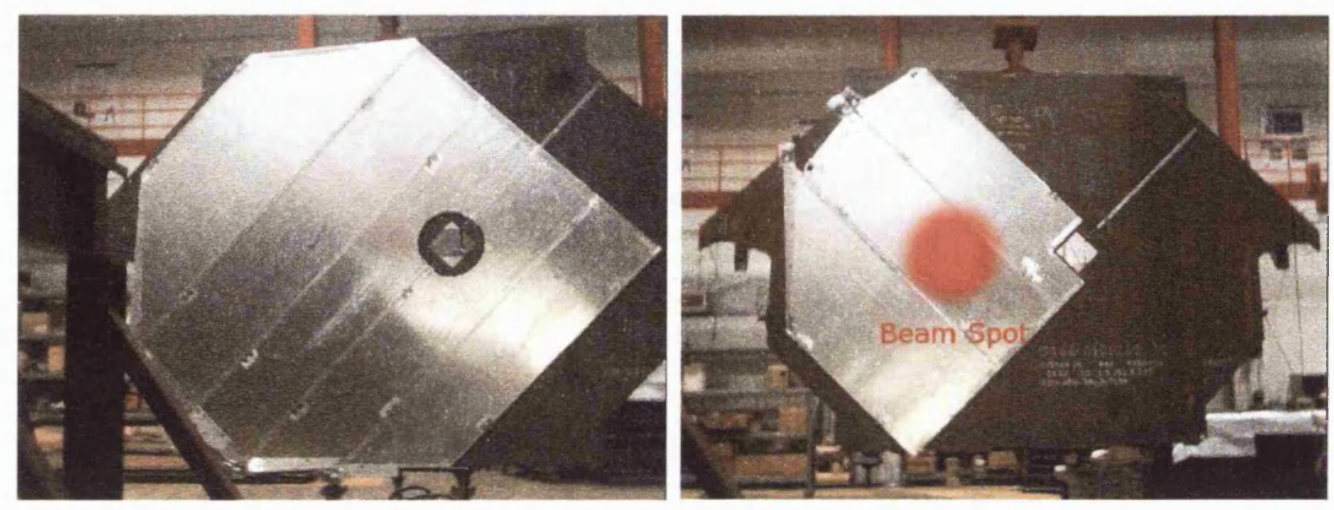

Figure 4.7: On the left is a fully instrumented Near Detector plane, and on the right is a partially instrumented plane. Both the coil hole and the centre of the beam are offset $50 \mathrm{~cm}$ from the centre of the plane.

It is possible to divide the detector into four logical sections, as illustrated in Figure 4.8. The first of these sections is the 'veto region', and consists of 20 planes of steel and scintillator. Neutrino interactions detected in this region of the detector will not be used in the oscillations analysis, to avoid possible end effects and neutron contamination.

The second section is the 'target region', it consists of 40 steel-scintillator planes. Beam neutrinos must interact in this section to be used in the near-far comparison. The next part is the 60 plane long hadron shower section. This region is long enough to contain the full showers of every neutrino interaction in the target region.

The final 160 planes in the detector form the muon spectrometer. The four metres of steel contained in this region is sufficient to obtain muon momenta measurements of similar accuracy to those made at the Far Detector.

Due to the small size of the beam spot, and the fact that the transverse spread of the hadronic showers in the detectors is only about $0.5 \mathrm{~m}$, it is not necessary 


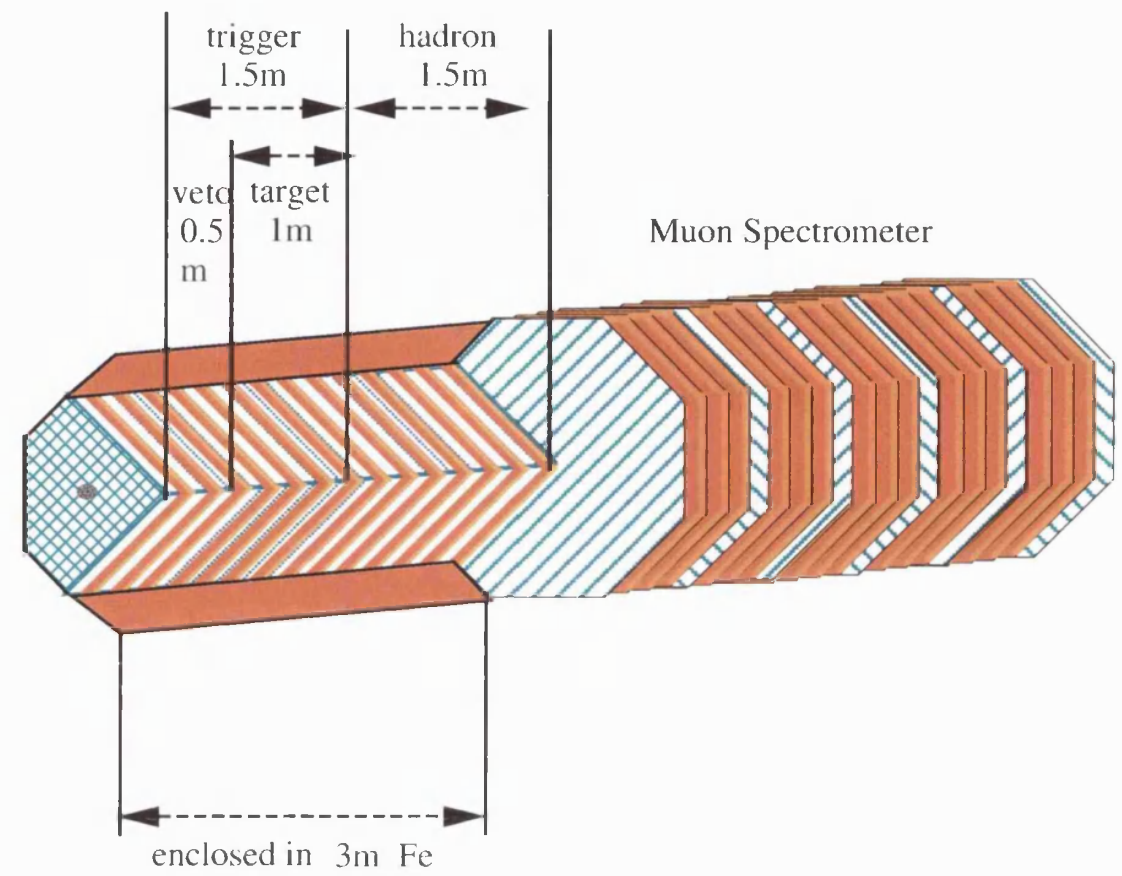

Figure 4.8: The instrumented regions of the Near Detector. The distances given are in terms of thickness of steel, and not actual length of detector.

to fully instrument each plane. In the veto, target and hadron shower sections only every fifth plane is fully instrumented, the other four planes only have one quadrant instrumented, see Figure 4.7. Due to the shape of the Near Detector planes this quadrant is rather more than a quarter of the total area of the plane. Every fifth plane is fully instrumented to measure muons which wander out of the instrumented region, and to detect other interactions in the non-instrumented region. In the muon spectrometer section we are only interested in measuring the muon momenta, through range or curvature, so it does not need to be as fine grained as the other regions. As such only every fifth plane is instrumented, with a full plane of scintillator, with the other four planes being uninstrumented.

In contrast to the Far Detector the scintillator strips in the Near Detector are only read out on one side, with the other end of the strip having a reflective surface. Due to the shorter strips and higher event rates, optical multiplexing is not used. Instead each scintillator strip is read out by one pixel of a Hamamatsu M64 multi-anode PMT, where each phototube has 64 pixels. Due to the high rates in the 
detector, high speed QIE electronics are used. The QIE system is a deadtime-less, multi-ranging ADC system which has been used in the KTEV and CDF experiments. The electronics divide the charge from the PMTs into 19 ns time buckets, digitizing each bucket separately. These time buckets must then be recombined during the reconstruction process. Whilst this is a potential source of systematic difference it is believed the electronics will be well enough understood, particularly after both sets of electronics have been used at the Calibration Detector.

\subsection{Physics Goals and Capabilities}

The goal of the MINOS experiment is to decisively prove whether neutrino oscillations are the cause of the atmospheric neutrino anomaly, and if they are to measure the oscillation parameters to $10 \%$. To do this it will measure and compare the neutral current (NC) and charged current (CC) interactions, in the Near and Far Detectors. The dominant interactions are those with quarks (as to first order the cross-section scales with target mass and the quark masses are much greater than the electron mass) and can be expressed as

$$
\begin{array}{lll}
N C: & \nu+N \rightarrow \nu+X & (\nu+q \rightarrow \nu+q) \\
C C: & \nu_{l}+N \rightarrow l^{-}+X & \left(\nu_{l}+d \rightarrow l^{-}+u\right),
\end{array}
$$

where $N$ is a nucleon, $X$ is a hadronic final state and $l \in\{e, \mu, \tau\}$. The events in the detector can be divided into two broad categories, namely short and long events, as sketched in Figure 4.9. Long events are those which contain a muon in the final state, the majority of these are charged current $\nu_{\mu}$ interactions, with a small component of $\nu_{\tau}$ charged current interactions (where the tau decays via $\tau^{-} \rightarrow \mu^{-}+\bar{\nu}_{\mu}+\nu_{\tau}$ ). Short events are those which only include a shower, these include all of the neutral current events and the charged current events involving $\nu_{e}$ and most of the $\nu_{\tau}$ (where the short lived tau decays into an electron, $\tau^{-} \rightarrow \mu^{-}+\bar{\nu}_{\mathrm{e}}+\nu_{\tau}$, or to a hadronic state, for instance $\tau^{-} \rightarrow d+\bar{u}+\nu_{\tau}$ ).

The short/long event classification is used in the so called 'T-test', where the ratio of ratios, $\left(N_{S H O R T} / N_{L O N G}\right)_{N e a r} /\left(N_{S H O R T} / N_{L O N G}\right)_{F a r}$ is examined as a func- 

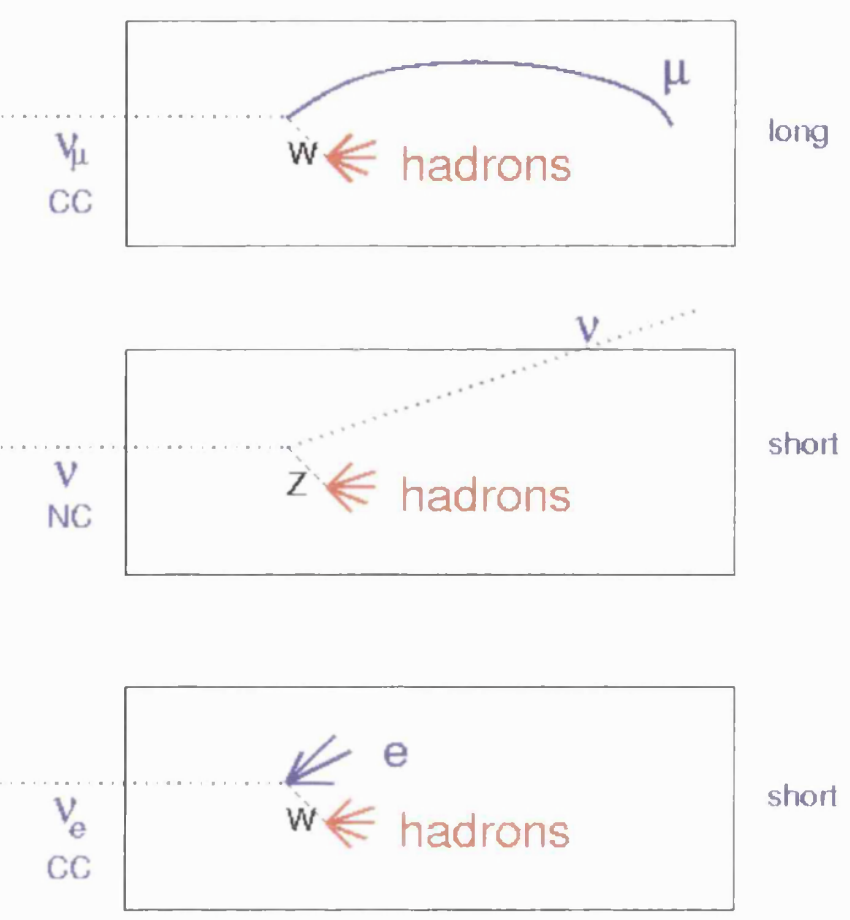

Figure 4.9: A sketch of short and long events in the MINOS detectors. There is an additional contribution to the long events from $\nu_{\tau}$ charged current events where the tau quickly decays via: $\tau \rightarrow \mu+\nu_{\tau}^{\prime}+\bar{\nu}_{\mu}$.

tion of energy. This is a useful quantity for two reasons: firstly it eliminates the problems due to differing rates in the detectors and secondly it is sensitive to oscillations both due to the disappearance of $\nu_{\mu}$ and also to $\nu_{e} / \nu_{\tau}$ appearance because the $\nu_{e}$ and most of the $\nu_{\tau}$ events will be short showering events. For maximal mixing this measurement can be sensitive to $\Delta \mathrm{m}^{2}$ as low as $10^{-3} \mathrm{eV}^{2}$ for $\nu_{\mu} \rightarrow \nu_{\tau}$ oscillations, as illustrated for two years running in Figure 4.10.

The T-test limits are dominated by statistical errors due to the low numbers of short events compared to long events, as many of the low energy short events will not pass the plane trigger requirements. The cross-section for the neutral current events rises sharply at low energy, so an accurate calibration will improve the statistics by allowing a lower low-end energy threshold. The T-test is a simple and statistically powerful test of the oscillation hypotheses. One strength of the method is that it is fairly immune to systematic errors, particularly those related to the flux and energy 


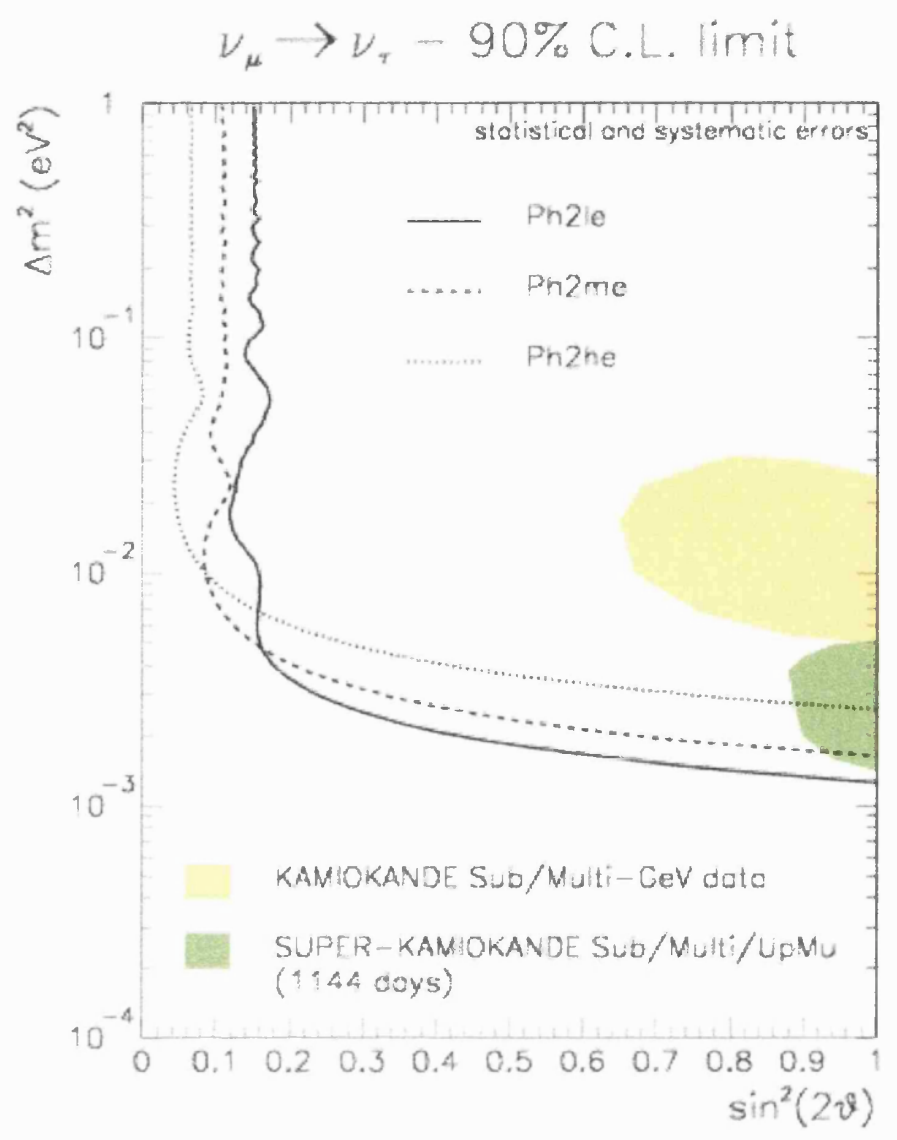

Figure 4.10: The $90 \%$ confidence limits on $\nu_{\mu} \rightarrow \nu_{\tau}$ oscillation parameters, from the 'T-test' method, for the three different beam configurations, assuming no evidence of oscillation is seen after two years of running. The Kamiokande and Super-Kamiokande preferred regions are shown.

spectrum of the neutrinos.

Whilst there are many merits to the T-test method, the most sensitive technique available to MINOS is using the $\nu_{\mu}$ charged current energy spectrum. In this method one measures the energy spectrum of long events at the Near Detector and then uses beam Monte Carlo to predict the spectrum at the Far Detector if there were no oscillations. Deviations between the measured Far Detector spectrum and the predicted spectrum are an indication that oscillations have taken place. This is illustrated in Figure 4.11, for three different values of $\Delta m^{2}$. The position of the dip in the spectra is given by $\Delta m^{2}$ and the magnitude of the dip is related to $\sin ^{2} 2 \theta$. 


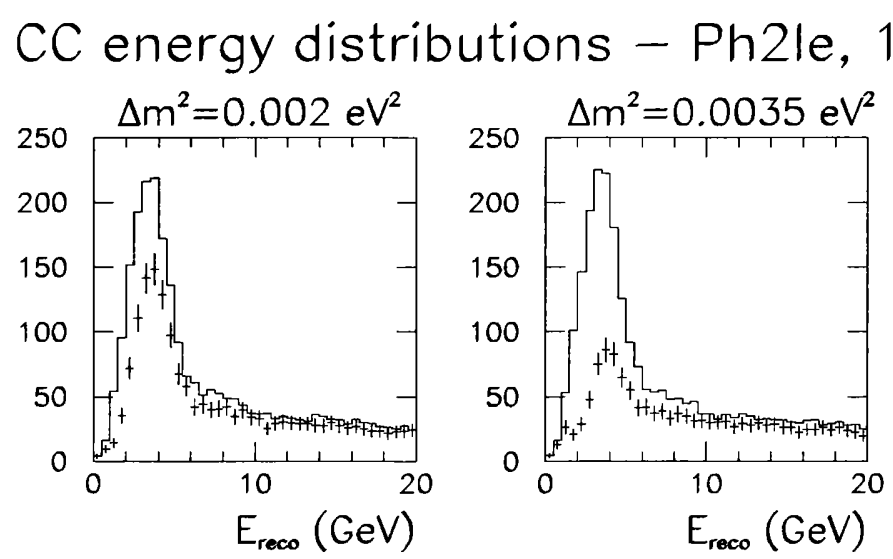

$10 \mathrm{kt} . \mathrm{yr} ., \sin ^{2}(2 v)=0.9$
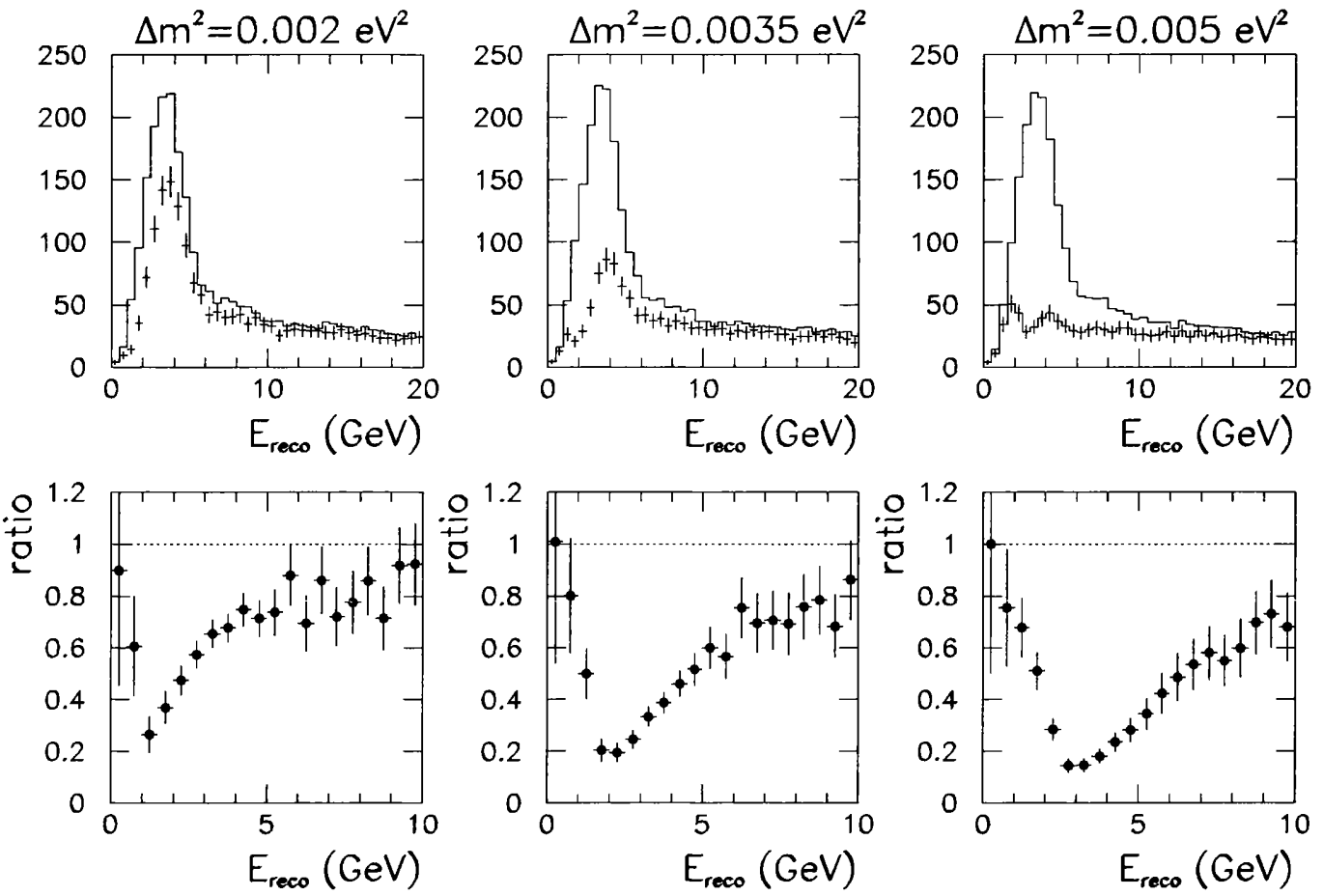

Figure 4.11: On the top are the oscillated and unoscillated energy spectra for charged current $\nu_{\mu}$ events, for three values of $\Delta m^{2}$. The spectra are plotted as a function of reconstructed energy $E_{\text {reco }}=E_{\mu}+E_{\text {Shower }}$. On the bottom is the ratio of these two spectra. The depth of the dip in the ratio is determined by $\sin ^{2} 2 \theta$, the position of the dip by $\Delta m^{2}$.

One advantage of this method, as discussed in [56], is that it is possible to measure the oscillation parameters without any dependence on energy scale calibration of the hadronic and electromagnetic showers. This is possible because the energy of the muons can be measured either through range, if the muon stops in the detector, or through curvature in the magnetic field. Using a simulation corresponding to 2 years of beam data it has been shown that the oscillation parameters can be measured to better than $30 \%$ using only the muon energy [56]. The limiting factor on this measurement is uncertainties in the $y$-distribution, the distribution of the fraction of neutrino energy transferred to the muon, in the $0-10 \mathrm{GeV}$ energy range. On average over this range the muon will have $\sim 65 \%$ of the observable energy and 
the hadronic component will be $\sim 30 \%$, with the remainder being electromagnetic. If this distribution was measured to a greater precision at these low energies then there would be no need for a hadronic energy calibration when measuring the $\nu_{\mu}$ energy spectrum. However, as this it not the case the best oscillation parameter measurement is obtained using the total visible energy to estimate the neutrino energy, from $E_{\nu}=E_{\mu}+E_{\text {Shower }}$. Using this method it should be possible to measure both $\Delta m^{2}$ and $\sin ^{2} 2 \theta$ to better than $10 \%$.

Both absolute and relative calibration are very important for this measurement; good absolute calibration is needed in accurately determining the position of the dip, and relative calibration is needed as the unoscillated spectrum is formed using Near Detector measurements of the beam. A good understanding of the beam is also essential to this measurement, as the Far Detector sees a much smaller fraction of the beam than the Near Detector. The $90 \%$ confidence limits, on $\nu_{\mu} \rightarrow \nu_{x}$ oscillation parameters, from two years of running, are shown in Figure 4.12. Assuming maximal mixing this technique is sensitive, in two years running, down to $\Delta m^{2} \sim 8 \times 10^{-4} \mathrm{eV}^{2}$.

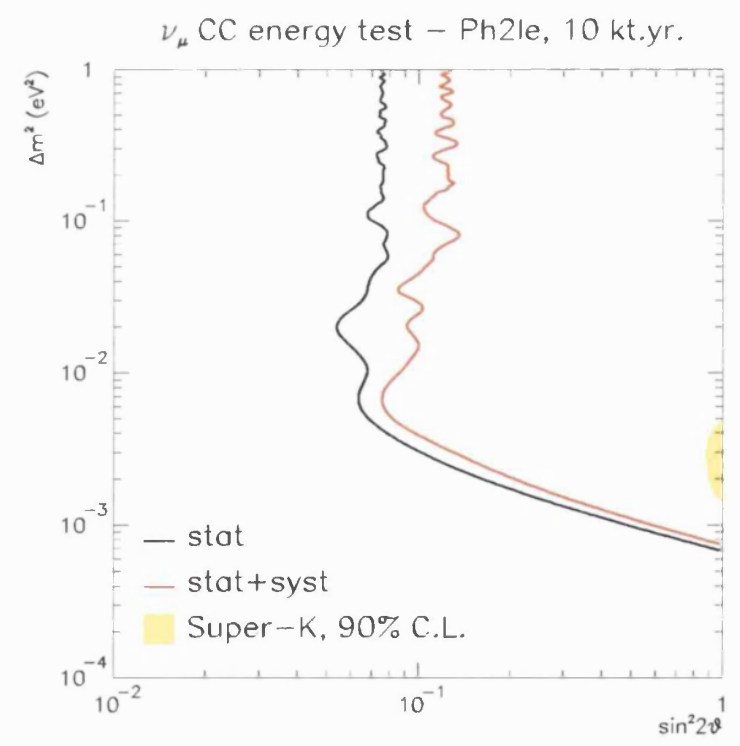

Figure 4.12: Limit plots showing the $90 \%$ confidence limits, from two years of running: assuming no oscillation signal is seen in the charged current $\nu_{\mu}$ energy spectrum. Beam systematics are included in this plot.

Using event topology and energy deposition, MINOS has some hadronic and 
electromagnetic shower distinguishing capability. Therefore $\nu_{e}$ appearance can also be investigated, in two years a modest improvement on the limits set by $\mathrm{CHOOZ}$ and Super-Kamiokande can be made, see Figure 4.13.

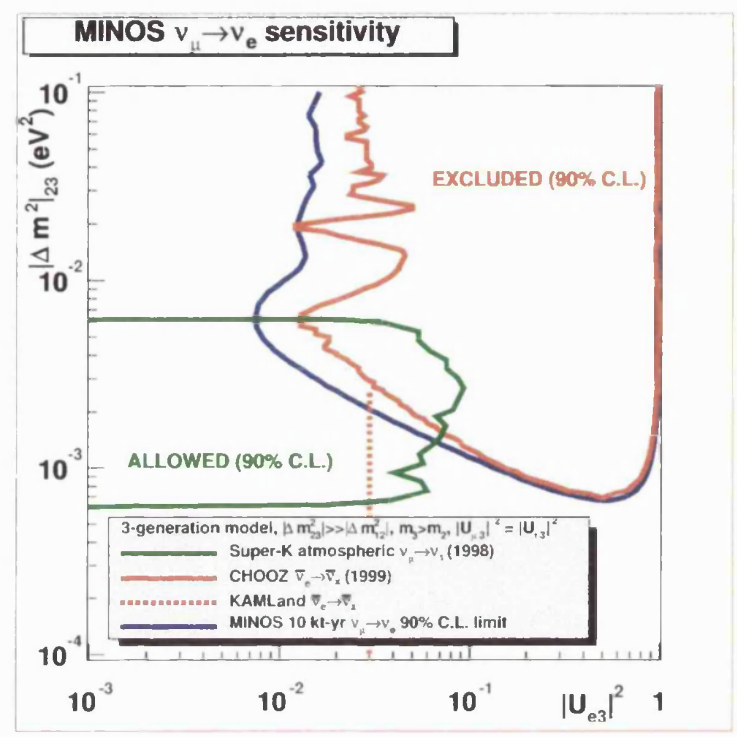

Figure 4.13: Limit plots showing the $90 \%$ confidence limits, from two years of running, assuming no oscillation signal from $\nu_{e}$ appearance. Modest improvements on the limits set by CHOOZ and Super-Kamiokande can be made. 


\section{Chapter 5}

\section{Overview Of The Calibration}

The MINOS experiment has an unusual, and also non-trivial, calibration dilemma; how do you accurately calibrate two large subterranean detectors, which are some $735 \mathrm{~km}$ away from each other? This chapter will explain why calibration is so important to the measurements that MINOS intends to make, and will outline the calibration procedures used. In the following chapters results from the calibration of both CalDet and the Far Detector will be shown.

\subsection{Introduction}

The MINOS detectors are large calorimeters and as such they measure the energy of particles. Events in the detector are characterized in terms of:

Muons The muon energy is determined either through curvature in the magnetic field or, if the muon stops in the detector, with better resolution using track length.

Hadrons Hadrons, typically protons and pions, tend to produce showers of particles in the detector. The energy of the hadron can be determined by summing the total signal observed in the event.

Electrons Electrons also produce showers of particles in the detector, electromagnetic and hadronic showers can be distinguished using event topology. The 
energy of the electron can also be inferred from the total signal seen in the detector.

The determination of muon energy is the least dependent on a calibration of the scintillator light output, assuming the light output is known to a sufficient level that the efficiency of muon detection is understood. The three MINOS detectors are of very similar design, meaning that muon energy measurements via range, or curvature, are directly transferable from one detector to another.

However, the energy scale calibration for both hadrons and electrons directly depends on the calibration of the scintillator light output. The signals observed in the three detectors from particles of identical energy differ dramatically. Calibration is needed to remove variations in: scintillator light yield; wavelength shifting fibre response and length; attenuation in the optical readout cables; optical transmission of connectors; response of the PMTs and electronics. It is only after all these effects are accounted for that energy deposits from, for example, $1 \mathrm{GeV}$ electrons are directly comparable between detectors (and indeed between different regions of one detector). This process of "flattening" the response of the three detectors is the relative calibration of the detectors.

The final stage of the calibration is to convert the calibrated signals from electrons, muons and hadrons into an actual energy. This is the absolute calibration; it is performed separately for the three particle types.

\subsection{Why Is Calibration Important?}

All of the oscillation measurements, from beam neutrinos, that MINOS will make rely on comparing events in the Near Detector to those in the Far Detector. This is the crux of any determination of the oscillation parameters that MINOS will make. For this reason it is essential that any differences between the two detectors be understood and calibrated out.

It is not only the relative calibration between the detectors that is important, the absolute energy calibration is also critical in the determination of oscillation 
parameters. Take for example the most sensitive measurement that MINOS can make; that of comparing the charged current $\nu_{\mu}$ energy spectrum at the Far Detector to the unoscillated spectrum extrapolated from the Near Detector, as shown in Figure 4.11. The charged current spectrum is formed from the combination of hadronic and muon energy in the detector: $E=E_{\mu}+E_{\text {Hadron }}$. As mentioned in section 4.3 , the position of the dip in this spectrum is determined by $\Delta m^{2}$ and the magnitude of the dip by $\sin ^{2} 2 \theta$. If the absolute energy scale is poorly calibrated this will directly effect the measurement of the position of the dip and hence the value of $\Delta m^{2}$.

In order to accurately determine the oscillation parameters, the MINOS detectors have been designed to achieve:

- A relative calibration of the energy scale of the Near and Far Detectors to $2 \%$.

- A calibration of the absolute energy scale to $5 \%$.

These values were chosen such that the systematic uncertainties on parameter measurement were smaller than the statistical uncertainty. It is thought that MINOS will be able to obtain sufficient statistics in order to make a $10 \%$ measurement of the oscillation parameters. As discussed above, $\Delta m^{2}$ is determined by the position of the dip in the energy spectrum and as such the determination is directly related to the absolute energy calibration. To ensure that the systematic contribution to the error on $\Delta m^{2}$ is minimized, an upper limit of $5 \%$ uncertainty on the absolute calibration was set.

The reasons for the $2 \%$ value for relative calibration are somewhat more vague and convoluted. There are two main sources of uncertainty when comparing the measured energy spectra at the Near and Far Detectors: the actual neutrino fluxes at the two detectors and the relative calibration between them. As discussed in Chapter 4, the MIPP experiment will help to reduce the uncertainty in Far Detector neutrino spectrum, as extrapolated from the Near Detector measurements, to the $2 \%$ level. A number of studies have investigated the effect of an energy scale miscalibration between the Near and Far Detectors [57][58][59]. These show that a shift in the energy scale between the Near and Far Detectors effects the determina- 
tion of both $\Delta m^{2}$ and $\sin ^{2} 2 \theta$, as well as influencing the quality of the fit. The $2 \%$ limit was selected so that:

i) The change in the best values for the oscillation parameters is small relative to the expected statistical error, i.e. the best fit point does not leave the nominal $90 \%$ confidence limit contour.

ii) The shift does not distort the energy spectrum such that the $\chi^{2}$ from the fit for the oscillation parameters becomes large, i.e. the shifts on a bin by bin basis are smaller than the statistical uncertainties.

Note the $2 \%$ value refers to the relative energy scale calibration between the Near and Far Detector. For muons, whose energy is determined either through range or curvature in the magnetic field, little use is made of the visible energy in the detector except as a cross-check. However for electrons and hadrons, who contribute $\sim 30 \%$ to the $\nu_{\mu}$ charged current spectrum, this $2 \%$ value relates to the calibration of the light output of the scintillator strips.

\subsection{Relative Calibration}

As anybody who has any experience of calibrating a detector will know, calibration to the $2 \%$ level is non-trivial. In MINOS this problem is made yet more difficult by the fact that we are calibrating the energy scales of our two detectors, separated by $735 \mathrm{~km}$, to $2 \%$.

The relative calibration relies on flattening the response of each detector, i.e removing any strip to strip and time dependent variations in the response of the detector. Muons are the main tool used to achieve this, with a light injection system to account for changes on a shorter timescale. The cross-detector calibration also uses muons. The energy deposits from muons of known energy are used to calibrate the relative response of the detectors. Starting with the electronics of a single channel and working up to the cross-detector calibration, the various stages of calibration will be outlined. 


\subsubsection{Charge Injection}

Each electronics channel has an inbuilt calibration mechanism, which can be used to linearize the response of the electronics to charge. A controlled quantity of charge is introduced at the input to the electronics, i.e. where the output from the PMT would go, this charge is then digitized. It is then possible to linearize the ADC scale using this known quantity of charge.

Charge injection is not necessary for the electronics channels connected to PMTs, as the light injection system can linearize the response of both the PMTs and the electronics, see section 5.3.2. However, it is essential for linearizing the response of the PIN diode electronics used in the light injection system.

\subsubsection{Light Injection}

The light injection system [60] has three main uses:

- To linearize the response of the PMT/electronics.

- To monitor short timescale drifting of this response.

- To accurately determine the position of the single photoelectron peak.

The system is the main hardware based calibration technique used in MINOS. Figure 5.1 shows, schematically, the components of the light injection system. The principle behind the system is to inject a carefully monitored amount of light into the standard optical readout path, and calibrate the response. Light from an ultraviolet LED is distributed across as many as 70 optical fibres in a pulser box. Most of these fibres transport the light from the LEDs to the end of the scintillator modules. On the end of a scintillator module is the light injection manifold, where the light from the fibre illuminates up to 10 of the wavelength shifting fibres in the module. From this point onwards the injected light follows the same path as light from the scintillator.

Two of the fibres that are most brightly illuminated by the LED in the pulser box transport the light to PIN diodes. These diodes are used to independently monitor the amount of light produced by the LED, two PIN diodes are used to 


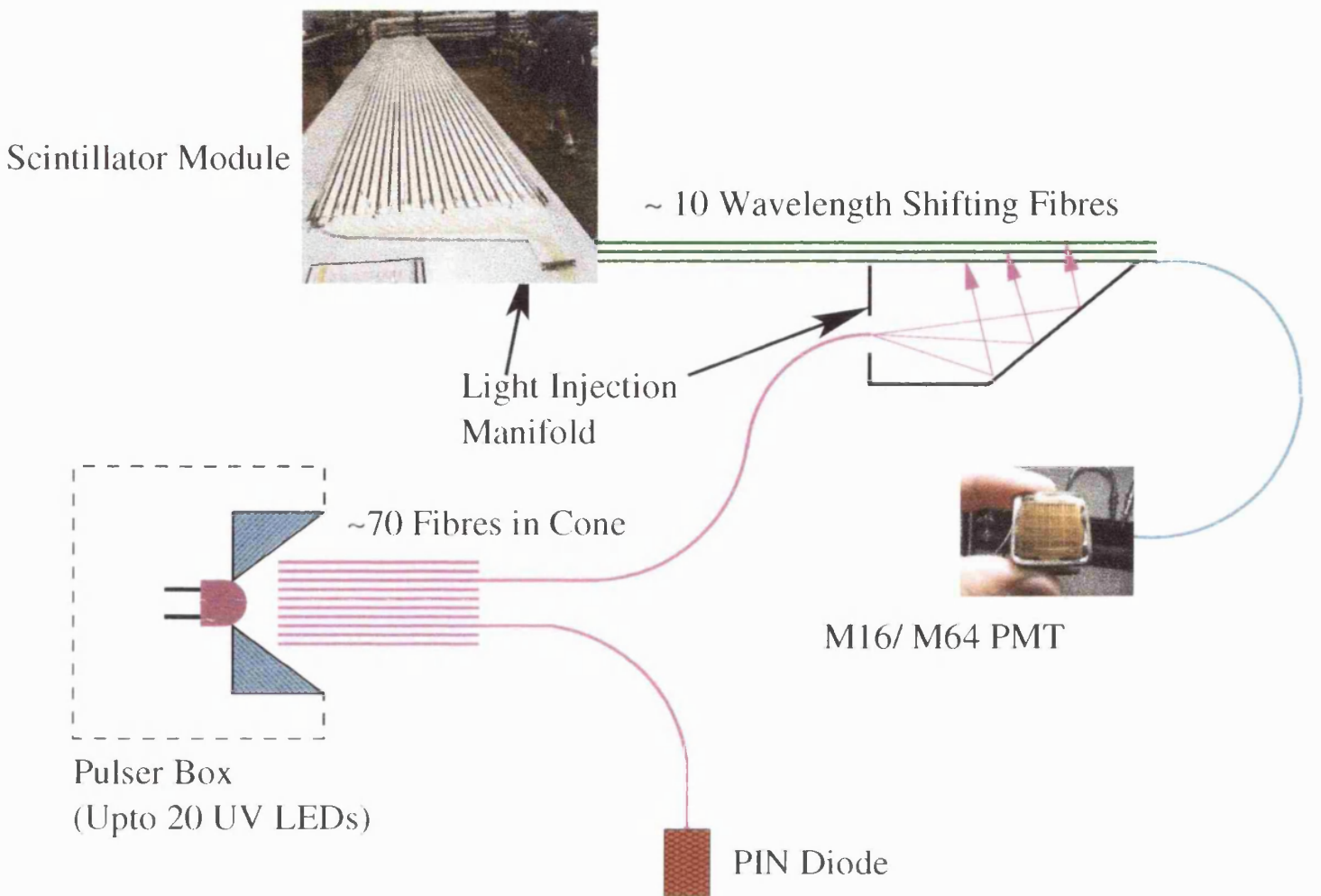

Figure 5.1: A schematic diagram of the light injection system. Light from ultra-violet LEDs illuminates up to 70 fibres in a pulser box, these fibres transport the light to the light injection manifolds at the ends of scintillator modules. In these manifolds the light is maximally dispersed across 8 or 10 wavelength shifting fibres. From this point the injected light follows the same path as the light from the scintillator. PIN diodes are used to monitor the light output of the LEDs.

provide a degree of redundancy. PIN diodes were chosen to monitor the light level as they have been shown to be stable to $0.5 \%$ over a two month period and no evidence of non-linearity has been observed [60]. Signals from the PIN diodes are shaped to look like PMT signals, and then digitized in the usual manner.

There is a large degree of flexibility built into the system with regards to pulsing the LEDs. The frequency, width and height of the pulses are all configurable. This flexibility enables the light injection system to be used for a number of purposes, all of which will be illustrated with results from the CalDet in chapter 6 . 
To linearize the response of the PMTs and electronics, light is injected at a range of different levels. Using the PIN diode to monitor the light level it is possible to convert the ADC scale for the electronics into a scale which is linear in light. Runs are taken to map out these linearization curves on the order of once a month.

Probably the most important function of the light injection system is to account for short timescale drifting of the response of the PMTs and electronics. This is particularly necessary at the Far Detector where the rate of cosmic ray muons is very low, approximately 500 per strip per month, so a system is needed to account for drifting responses while muon statistics are being accumulated. To account for this drift the LEDs are flashed at a constant height on the order of once per hour. Then the PMT/PIN response is compared to a reference point and any drift is removed.

Whilst not being strictly part of the calibration chain, determination of the single photoelectron peak is very useful, as it determines the ADC to photoelectron (PE) scale. This is essential if one is to use the Poissonian nature of photodetectors in any analysis. Special runs are taken using the light injection system, to determine the position of this peak for every pixel (and indeed every pixel spot).

\subsubsection{Strip To Strip Calibration}

Differences in the response of the strips, within each detector, are removed using muons. This strip to strip calibration accounts for:

- Scintillator light yield.

- Wavelength shifting fibre differences.

- Optical connections.

- Attenuation of readout cables.

These calibration constants are produced by taking either the peak or mean of the muon ADC spectrum in each strip [56].

There are a number of effects which must be taken into consideration when the muon ADC spectra are constructed. The three main effects are: the distributions of 
muon energy, path length in scintillator and transverse position along the scintillator strip. These are important as they effect the amount of energy deposited in the strips, and if the distributions differ from strip to strip within a detector then this can lead to systematic errors in the calibration constants. One way to minimize these errors is to select a sample of muons for which the distributions are as similar as possible in the strips to be calibrated. The other method is to correct for these effects on a hit by hit basis.

For the fiducial region of the Far Detector nearly all cosmic muons can be used in the calibration, as they are predominantly high energy through-going muons and the angular distribution and strip population is similar between the two plane orientations. At the Calibration Detector the angular distribution of cosmic muons in the two orientations is very different, the difficulties that arise from this will be discussed in section 7.2.

\subsubsection{Cross-Detector Calibration}

Muons are also used to calibrate the relative energy scale across the three detectors. The energy of individual muons is not important for the strip to strip calibration within a detector, as long as the energy spectrum is the same for all strips to be calibrated. However, for the cross-detector calibration only muons of known energy are used. This is necessary due to the difference in cosmic muon energy spectra between the three detectors; at the Far Detector the muons are much more energetic on average than those in the other two detectors due to its location at the bottom of the mine.

The energy of the muons is determined either through their curvature in the magnetic field or, with better resolution, by range if they stop in the detector. In the Far Detector it is only possible to measure the energy of muons if they are less than $\sim 50 \mathrm{GeV}$. As the Calibration Detector has no magnetic field, stopping muons must be used. The best sample of stopping muons is beam muons of less than $2 \mathrm{GeV}$, more energetic muons traverse the entire length of the detector.

Using these muons of known energy it is possible to cross-calibrate the detectors 
in terms of a standardized muon energy unit (MEU).

\subsection{Absolute Calibration}

The final step in the calibration chain is converting from the standardized muon energy units to actual energy in $\mathrm{GeV}$. The standard technique for calibrating the energy scale of a calorimeter is to expose it to a beam of particles of known energy. Obviously due to the size and location of the Near and Far Detectors, this is not possible for MINOS. Instead of exposing the detectors themselves, a smaller Calibration Detector was constructed, see Chapter 6 . The purpose of this detector is to characterize the response of electrons, hadrons and muons of known energies in the MINOS detectors in terms of MEU and event topology. Using this information it is possible to calibrate events in the Near and Far Detectors in terms of GeV. 


\section{Chapter 6}

\section{The Calibration Detector}

The Calibration Detector (CalDet) was constructed in 2001, and has spent the last three years collecting data in a series of testbeams at CERN. The detector was designed to achieve a $5 \%$ absolute energy scale calibration of the two other MINOS detectors. It has also proved a very useful testbed for detector components and calibration procedures. This chapter will explain the motivation for building a Calibration Detector and describe the characteristics of the detector.

\subsection{Why a Calibration Detector?}

The main MINOS results will come from comparing measurements of the neutrino beam made at the Near and Far Detectors. To achieve accurate measurements of the oscillation parameters, both the relative (Near-Far) and absolute energy scale calibrations must be very good. To achieve this the CalDet was designed and constructed. It is not possible, due to their size and location, to calibrate the Near and Far Detectors by exposing them to beams of particles of known energy, in the standard method of calibrating a calorimeter. Instead the CalDet was constructed as a surrogate detector that could be exposed to beams of hadrons, muons and electrons, and characterize their response in the MINOS detectors. This characterization can then be transferred to the Near and Far Detectors, after a relative calibration between the three detectors using muons. 
The CalDet has also provided a very useful testbed for components of the detectors, in particular the electronics. Both the Near and Far Detector electronics had their first large scale test at CERN with the CalDet, problems solved in these tests have greatly smoothed the installation of the detectors. It is not only the hardware components that have benefitted from the testbeam running, the procedures for calibrating the detectors have been tested and refined.

\subsection{Description of CalDet}

The CalDet was designed to be as similar as possible to the Near and Far Detectors. Perhaps the most useful way to view the detector is as a $1 \mathrm{~m} \times 1 \mathrm{~m} \times 3.6 \mathrm{~m}$ section of one the larger detectors. The detector is $\sim 12 \mathrm{~T}$ and as such is large enough to contain showers from electrons and hadrons of similar energy to those produced from beam neutrino interactions in the Near and Far Detectors. The thickness of the steel and scintillator planes along with the pitch of the planes are, within tolerances, identical to those of the Near and Far Detectors. The detector consists of 60 planes of steel and scintillator, with each scintillator plane subdivided into 24 strips of width $4.1 \mathrm{~cm}$. To obtain a three dimensional readout each successive plane of scintillator is rotated by 90 degrees. At the CalDet this results in scintillator strips that run horizontally and vertically, in the two other detectors both orientations of scintillator strip are at $45 \%$ to the horizontal. The other major difference between CalDet and the two other detectors is that the CalDet is unmagnetized. Studies show that the magnetic field has a negligible effect on the characteristics of electromagnetic and hadronic showers [61]. The effect of magnetic fields on the PMTs have also been studied [62][63][64]. Inside the metal boxes that house the PMTs the residual field from the magnetized detectors is expected to be less than 5 Gauss. At these low fields the properties of the PMTs are largely unchanged, although there is a slight decrease in the collection efficiency of edge pixels.

Figure 6.1 shows the Calibration Detector in the T11 testbeam area at CERN, and the two orientations of scintillator planes are clearly visible. Also visible are 


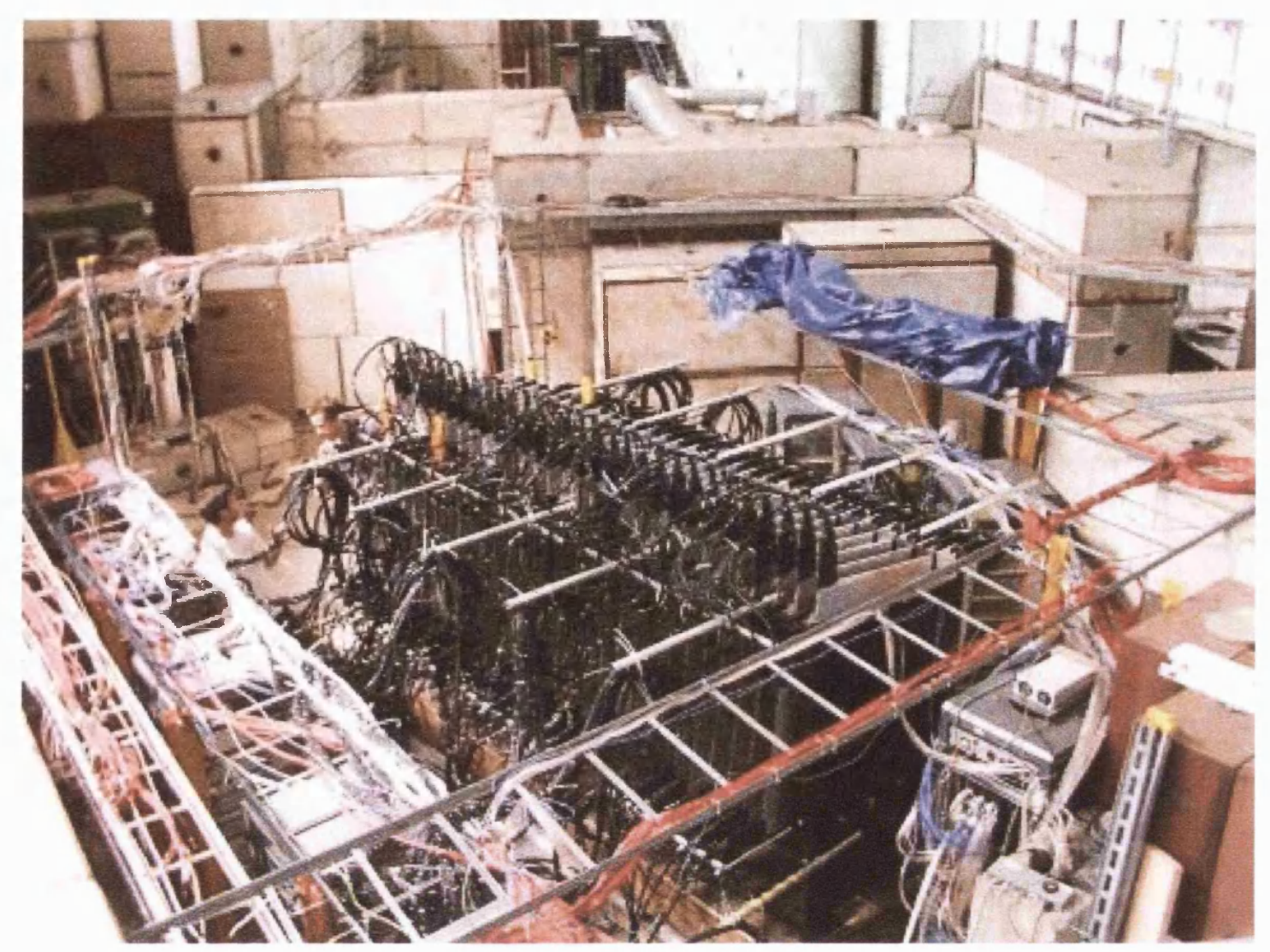

Figure 6.1: The Calibration Detector in the T11 testbeam area at CERN. Each successive plane of scintillator in the detector is rotated by $90^{\circ}$ to provide a three dimensional tracking capability.

the optical readout cables that transport light from the scintillator modules to the Hamamatsu multi-anode PMTs. In the initial period of running M16 PMTs and Far Detector electronics were used to digitize the light from the detector. In this period half of the readout cables were $6 \mathrm{~m}$ long clear optical fibre cables, like those used in the mine, and the other half were $4 \mathrm{~m}$ long green wavelength shifting fibre cables, to simulate the $8 \mathrm{~m}$ scintillator planes used in the Far Detector. During the running with Near Detector electronics, only one side of the scintillator strips were read out, with reflector connectors on the other end mimicking the Near Detector.

The bulk of the results from the CalDet presented in this thesis are from the running with Far Detector electronics. The standard mode of these electronics is the dynode trigger mode, where any PMT with a signal on the dynode (1 dynode 
corresponds to 16 pixels) greater than some threshold value, nominally one third of a photoelectron, is digitized. Each of the 22 channels on the VA chip associated with that PMT are digitized; 16 of these correspond to the PMT pixel, one further channel is associated with the PIN diode and the five remaining channels are floating, they are used for common mode noise correction. Up to 36 PMTs are read out by one VA Readout Controller (VARC), whose job it is to coordinate the digitization and time-stamping of the channels, each channel on a PMT is timestamped with time of the dynode trigger. After digitization, the VARC performs pedestal subtraction, common mode correction and zero sparsification on the digits. The zero sparsification removes signals below a certain threshold to reduce data rate. All 22 channels on the VA chip are read out with each dynode trigger, but normally only one or two of these channels have signals above threshold from energy deposits in the detector. This means that the zero sparsification results in a factor of $10-20$ reduction in the data rate.

The remaining digits are read out over VME into the DAQ computers. Once in the DAQ the digits are time sorted, and some event construction and triggering may be applied. Events are constructed by looking for gaps between successive hits greater than some threshold time, typically $200 \mathrm{~ns}$, the gaps mark the boundaries between events. These events may then be subject to a triggering requirement before being written to disk. A standard trigger is the plane trigger, where $M$ out of $N$ consecutive planes must have a digit before the event is written out. However, so as not to lose any information most of the beam data taken did not have any DAQ trigger applied.

One problem of using the Far electronics is that they were designed for the low rate environment of the Soudan mine. As such they have a deadtime of between 5$30 \mu$ s following each readout of the PMTs. At the mine the single PMT trigger rate is $\sim 1 \mathrm{kHz}$, this results in $\sim 2 \%$ total deadtime. However, in the CERN testbeams the rate is much higher, $>20 \mathrm{kHz}$ during the spills, and hence the deadtime fraction is also much higher. To overcome this problem a large amount of the testbeam data was taken using an external hardware trigger, described in section 6.2.1. 
Very little data has so far been taken using the Calibration Detector with Near Detector electronics. A substantial period of running using both electronics simultaneously, and just Near electronics, is scheduled for Autumn 2003.

\subsubsection{CERN Testbeams and Particle Identification}

The CalDet was constructed at CERN for use in the East Area testbeams. The detector has been exposed to both the T11 and T7 test beams, between these two beams energies in the range $0.2-10 \mathrm{GeV}$ have been investigated.

The testbeams are formed from protons from the Proton Synchrotron (PS) incident on a user selectable target. The secondary particles from these interactions are then focused and undergo momentum selection through a series of magnets. This results in a beam primarily of pions, protons, electrons and muons, the muons are formed from the decay of pions and so are not mono-energetic like the other particles.

The CalDet has two forms of particle identification: Cerenkov detectors and a time of flight (TOF) system. The gas and pressure of the Čerenkov detectors can be adjusted so that only electrons, or electrons and other particles, trigger them. The TOF system consists of three small scintillator paddles in the beamline upstream of the CalDet, the system is primarily used to distinguish protons from pions. Using a combination of these devices it is possible to produce samples of electrons, protons and pions, with some muon contamination in the pion sample.

Due to the high rates and long deadtime of the Far electronics, along with some interesting beam systematics in the $\mathrm{T} 7$ beam, a hardware trigger relying on the time of flight trigger was instigated. In this mode of running the electronics were only enabled by a coincidence in the TOF system. Running in this mode the detector live time is maximized during the beam spill, and only events where there is good particle identification information are recorded.

During the testbeam running, cosmic ray muons and high energy muons coming from the PS, but not down the beam pipe, are also recorded. These particles are very useful for checking the full functionality of the detector following each move 
into the testbeam areas. Between testbeam runs the detector is situated in a wooden enclosure in the same hall as the testbeams. In this area it is also able to take data from cosmic ray and PS related muons. 


\section{Chapter 7}

\section{The Calibration of the Calibration}

\section{Detector}

The Calibration Detector was the first of the three MINOS detectors constructed. As such all of the calibration tools and techniques employed by MINOS were developed or tested, on the CalDet. In this chapter work undertaken in calibrating the detector is detailed. This work is essential for the measurements of hadronic and electromagnetic showers in the CalDet to be used in calibrating the other MINOS detectors.

Methods for determining the position of the single photoelectron peak of PMT pixels are discussed. Results from each of the three main steps in the relative calibration chain are presented; the removal of time dependent electronic effects using the light injection system, the strip to strip calibration using muons and the relative calibration of the CalDet between the T11 and T7 test beams.

\subsection{Light Injection}

The light injection system used at the CalDet is functionally identical to the system used at the Far Detector, but utilizes only one pulser box with six LEDs. The CalDet was the first large scale test of the final MINOS light injection system. 
The system is used to determine the position of the single photoelectron peak, linearize the response of the PMTs/electronics and to account for drifts in their response over short timescales.

\subsubsection{Single Photoelectron Peak}

The position of the single photoelectron (PE) peak is a very important quantity when dealing with PMTs. Minimum ionizing particles (MIP), such as muons, on average produce 10-20 photons traversing a MINOS scintillator strip. After attenuation and collection efficiency effects this leads to only a few photoelectrons being created at the PMT photocathode. Since the number of photoelectrons is low, Poisson statistics must be taken into account when considering the resulting distributions; in particular the muon energy distributions used in strip to strip calibration. To achieve this the relationship between ADC and PE must be determined, i.e. the position in ADC of the single photoelectron peak. For most physics purposes a determination of this relationship to $5 \%$ is sufficient, although for high level sanity checking of the detector hardware a more precise determination is probably desirable.

Four methods for the determination of the position of the single photoelectron peak, or gain of the PMT and electronics have been investigated. Each method relies either on fitting to the peak directly, or calculating the mean light level, in terms of the number of photoelectrons (NPE), of a histogram and determining the gain through $G=\frac{m e a n}{N P E}$.

The first method calculates the light level using only the zero photoelectron contribution to the histogram (the pedestal) assuming Poisson statistics, see Figure 7.1. If a distribution follows Poisson statistics then the probability of obtaining $N$, when the mean value is $\lambda$, is given by

$$
P(N)=\frac{e^{-\lambda} \lambda^{N}}{N !}
$$

So the probability of having an entry in the pedestal, where $N=0$, is $e^{-\lambda}$, meaning 
the gain can be calculated from

$$
G=\frac{\mu}{\log \frac{N_{t o t}}{N_{p e d}}},
$$

where $\mu$ is the histogram mean, $N_{\text {tot }}$ and $N_{\text {ped }}$ are the number of entries in the histogram and pedestal respectively. Obviously this method is only effective when the pedestal is present in the distribution, i.e. for light levels below $\sim 4 \mathrm{PE}$.

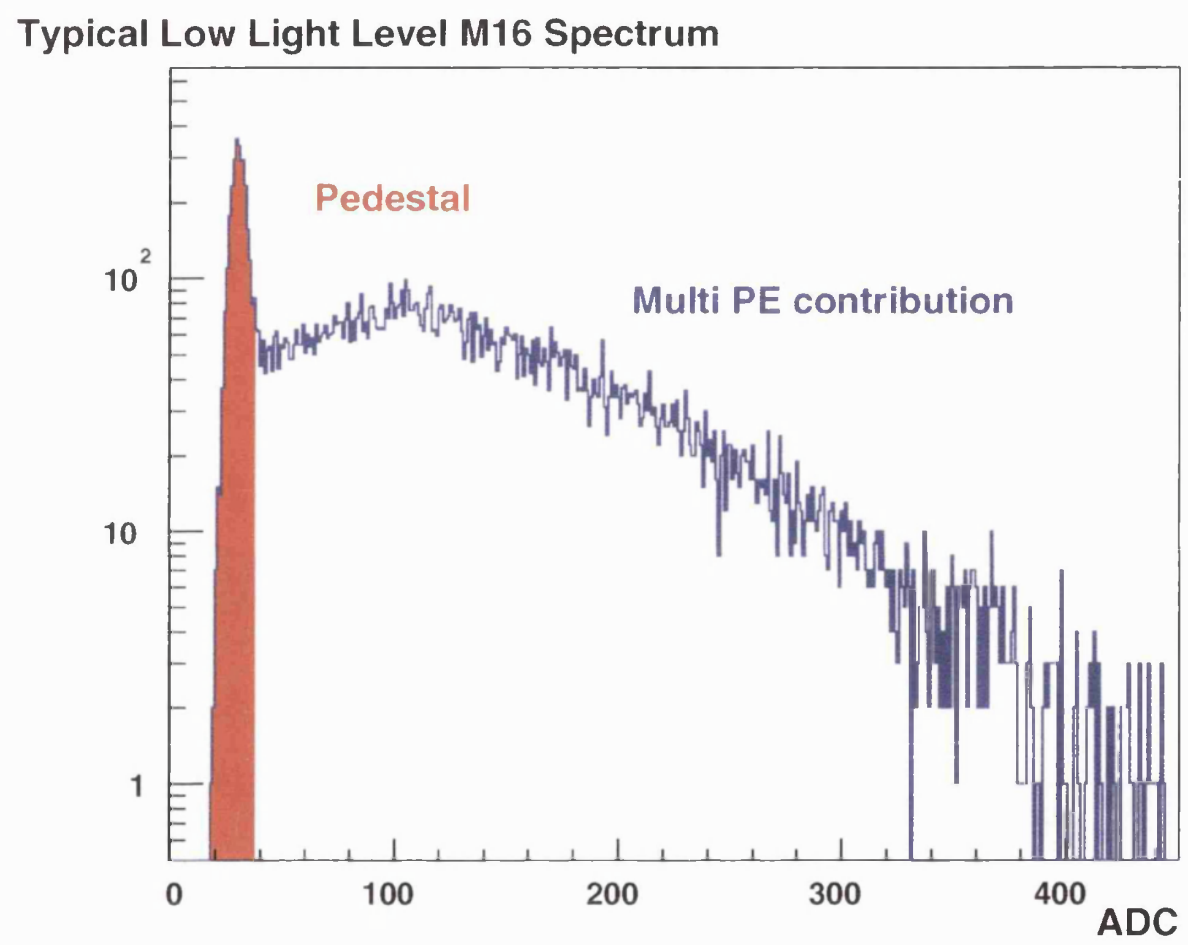

Figure 7.1: A low light level ADC distribution from a CalDet M16. The region in red is the pedestal, which is the zero photoelectron contribution to the distribution. The fraction of entries in the pedestal can be used to estimate the mean light level, and hence the gain.

The second and third methods derive the gain from a fit to the low light level ADC spectrum. The fit used was based upon the fit described in [65], and also from [66]. The fit assumes a Poissonian distribution of photoelectrons, with each photoelectron peak being described by a Gaussian with pedestal subtracted mean, 
$\mu_{n}$, and width, $\sigma_{n}$, given by

$$
\mu_{n}=n G
$$

and

$$
\sigma_{n}^{2}=n \sigma_{1}^{2}+\sigma_{p e d}^{2}
$$

where $\sigma_{1}$ is the width of the single photoelectron peak. An additional Gaussian term is used to describe photons which pass through the photocathode and produce a photoelectron at the first cathode, thus missing one stage of multiplication. This term was included to better match the shape observed in the data, which has more entries in the region between the pedestal and the single photoelectron peak than the original function described. Two fitted low light level M16 spectra, with the contributing Gaussians, are shown in Figure 7.2. Algebraically the function can be described as

$$
\begin{aligned}
f(x) & =N_{t o t} \frac{e^{-\lambda}}{\sqrt{2 \pi} \sigma_{p e d}} e^{-\frac{\left(x-\mu_{p e d}\right)^{2}}{2 \sigma_{p e d}^{2}}} \\
& +N_{t o t}\left(1-d_{f}\right) e^{-\lambda} \sum_{n=1}^{12} \frac{\lambda^{n}}{n ! \sqrt{2 \pi\left(n G \sigma_{1}^{2}+\sigma_{p e d}^{2}\right)}} e^{-\frac{\left(x-n G-\mu_{p e d}\right)^{2}}{2\left(n G \sigma_{1}^{2}+\sigma_{p e d}^{2}\right)}} \\
& +N_{t o t} d_{f}\left(1-e^{-\lambda}\right) \frac{1}{\sqrt{2 \pi\left(G \sigma_{1}^{2} / d_{s}+\sigma_{p e d}^{2}\right)}} e^{-\frac{\left(x-G / d_{s}-\mu_{p e d}\right)^{2}}{2\left(G \sigma_{1}^{2} / d_{s}+\sigma_{p e d}^{2}\right)}}
\end{aligned}
$$

where the fitted parameters are: $\mu_{\text {ped }}$ - the pedestal mean, $\sigma_{\text {ped }}$ - the pedestal width, $\lambda$ - the mean light level, $G$ - the position of the $1 \mathrm{PE}$ peak, $\sigma_{1}$ - the fractional width of the $1 \mathrm{PE}$ peak, $d_{f}$ - the fraction of photons passing through the photocathode and $d_{s}$ - the multiplication at the first dynode. The gain can be obtained from the fit either directly using the position of the single photoelectron peak, $G$, method 2 , or indirectly using the histogram mean divided by the fitted light level, $\lambda$, method 3 .

The final method for determining the gain relies on determining the light level using the photoelectron counting technique described in [65]. Assuming the initial number of photoelectrons at the photocathode is dominated by Poisson statistics 
Fitted Low Light Level Spectrum With Contributing Gaussians

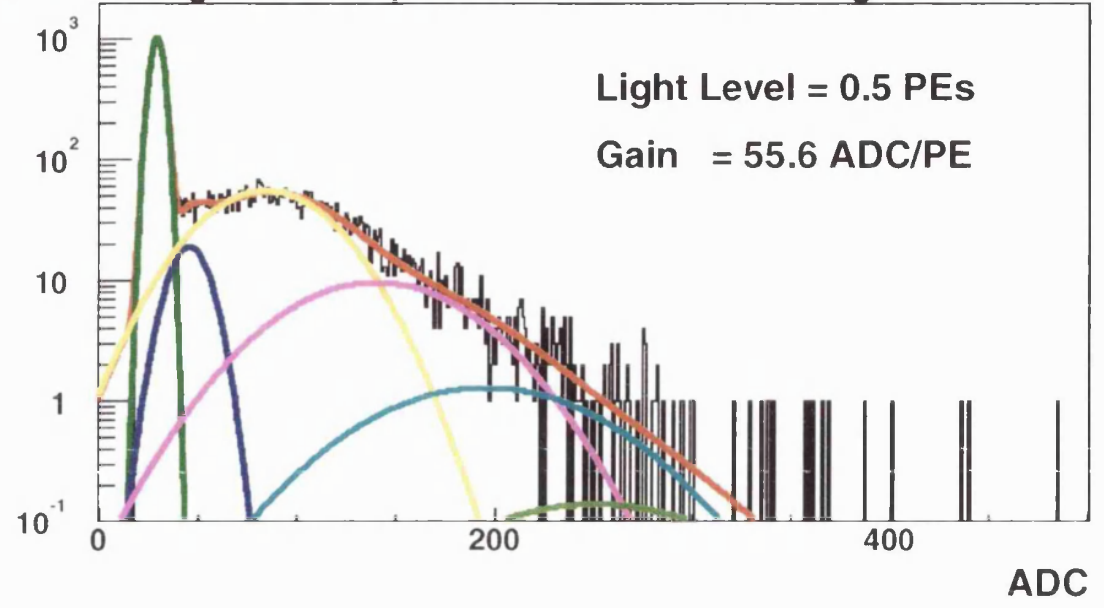

Fitted Low Light Level Spectrum With Contributing Gaussians

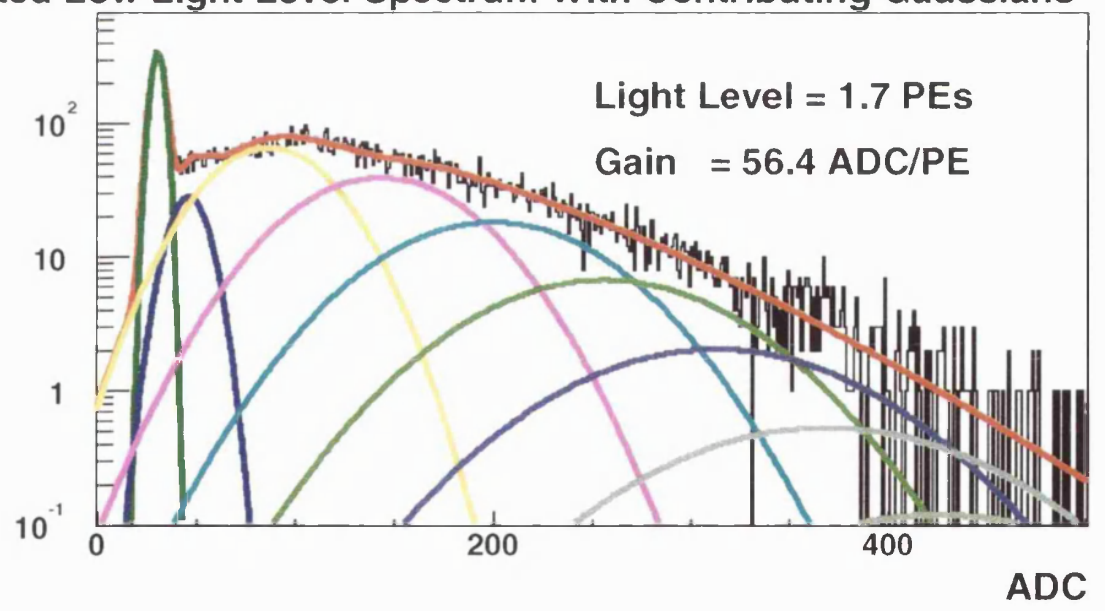

Figure 7.2: Two example fits, to the same channel, of the low light level ADC spectrum. The fit consists of Gaussians weighted by Poisson statistics for the pedestal and first 12 photoelectron peaks. The Gaussians for the highest photoelectron peaks are not visible on the logarithmic plot as they only contribute fractionally to the overall fit. An additional term is added to describe the case when a photon passes through the photocathode and produces an electron at the first dynode. The shape of the single photoelectron peak is not ideally described by a Gaussian, as it is asymmetrical. 
then

$$
\lambda=\left(\frac{\mu}{\sigma_{0}}\right)^{2},
$$

where $\mu$ is the mean of the pedestal subtracted ADC distribution and $\sigma_{0}$ is the RMS of the Poisson fluctuation at the photocathode multiplied by the gain G:

$$
\begin{gathered}
\mu=G \lambda \\
\sigma_{0}=G \sqrt{\lambda} .
\end{gathered}
$$

It is not possible to measure $\sigma_{0}$ directly, instead what is actually measured is the RMS of the ADC distribution, $\sigma_{0 m}$ :

$$
\begin{aligned}
\sigma_{0 m}^{2} & =\left(\sigma_{0}^{2}+\sigma_{1}^{2}+\sigma_{2}^{2}+\ldots \sigma_{\text {ped }}^{2}\right) \\
& =G^{2}\left(\lambda+\frac{\lambda}{g_{1}}+\frac{\lambda}{g_{1} g_{2}}+\ldots\right)+\sigma_{p e d}^{2}
\end{aligned}
$$

where $\sigma_{i}$ and $g_{i}$ are the RMS of the photoelectron distribution and the gain at the $i$ th dynode respectively. Using a similar argument the measured width of the single photoelectron peak, $\sigma_{1 m}$, is

$$
\begin{aligned}
\sigma_{1 m}^{2} & =\left(\sigma_{1}^{2}+\sigma_{2}^{2}+\ldots \sigma_{p e d}^{2}\right) \\
& =G^{2}\left(\frac{1}{g_{1}}+\frac{1}{g_{1} g_{2}}+\ldots\right)+\sigma_{p e d}^{2}
\end{aligned}
$$

where $\lambda$ is 1 as we are considering the single photoelectron peak. This leads to

$$
\sigma_{0 m}^{2}=G^{2}\left(1+\left(\frac{\sigma_{1 m}}{G}\right)^{2}\right)
$$

The measured RMS is larger than the true RMS due to fluctuations in the secondary emission at the first dynode. The light level can be determined through

$$
\lambda=\left(\frac{\mu}{\sigma_{0 m}}\right)^{2}\left(1+\left(\frac{\sigma_{1 m}}{G}\right)^{2}\right)
$$

using this light level the gain can be determined $G=\frac{\mu}{\lambda}$.

The advantage of this method of photoelectron counting, and therefore gain determination, is that it can be used at high light levels when no pedestal contribution is present. The disadvantage is that the fractional width of the single photoelectron 
peak is required. In practice, this means that another method must be used to determine the scale factor before this method becomes useful.

These four methods have been tested on data from the CalDet M16 PMTs. Low light level data was taken with the light injection system. Each PMT was illuminated with an average of between 0.3 - 4 photoelectrons over 15000 pulses. The gain was determined from the resulting ADC spectra using the four methods outlined above. The results of these methods on the $2500+$ channels in CalDet are shown in Figure 7.3. The histogram from the $(\mu / \sigma)^{2}$ method has been scaled to the same mean as the fit method, this accounts for the secondary emission broadening of the width described above. From previous measurements of the gain of M16 PMTs [67], we expect a $10 \%$ variation in the gain of pixels on a PMT, which is in good agreement with the measured width of the four methods.

The gain determined using the number of entries in the pedestal is systematically higher; due to an overestimation of the number of entries in the pedestal resulting from its non-Gaussian nature.

Using the data from the CalDet M16s it is possible to test the validity of Equation 7.12. One of the parameters of the fit is the fractional width of the single photoelectron peak. The ratio of the gain determined from the fit and the gain from $(\mu / \sigma)^{2}$ should be

$$
\frac{G_{f i t}}{G_{(\mu / \sigma)^{2}}}=\left(1+\left(\frac{\sigma_{1 m}}{G}\right)^{2}\right)^{-1} .
$$

These two quantities are plotted in Figure 7.4. The two distributions are similar, although the ratio of gains is wider than the predicted value. This is due to the two fold measurement error in the ratio, and also from the inherent non-Gaussian nature of the $1 \mathrm{PE}$ peak, meaning the width is not ideally modelled by the fit.

\section{Results from Simulation of Phototube Spectrum}

The four methods of gain determination have been applied to simulated spectra to investigate the systematic dependence of the methods on number of light pulses and light level. The simulation used was a modification of the simulation described 

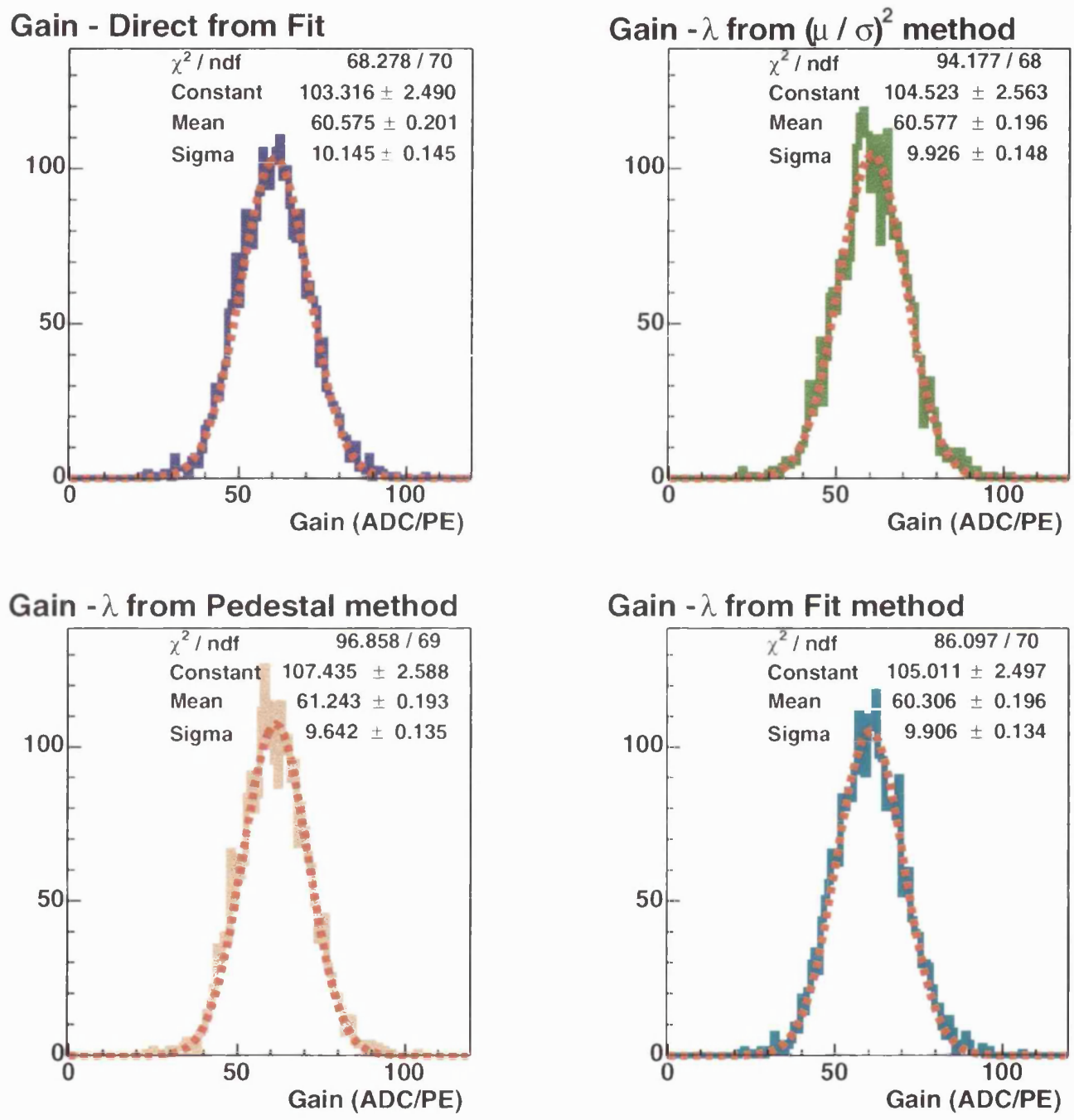

Figure 7.3: The gain of M16 pixels at the Calibration Detector, determined using four methods. The gains from $(\mu / \sigma)^{2}$ method have been scaled, by a constant factor, to the gain determined from the fit, this accounts for the effect of the secondary emission at the first dynode. 


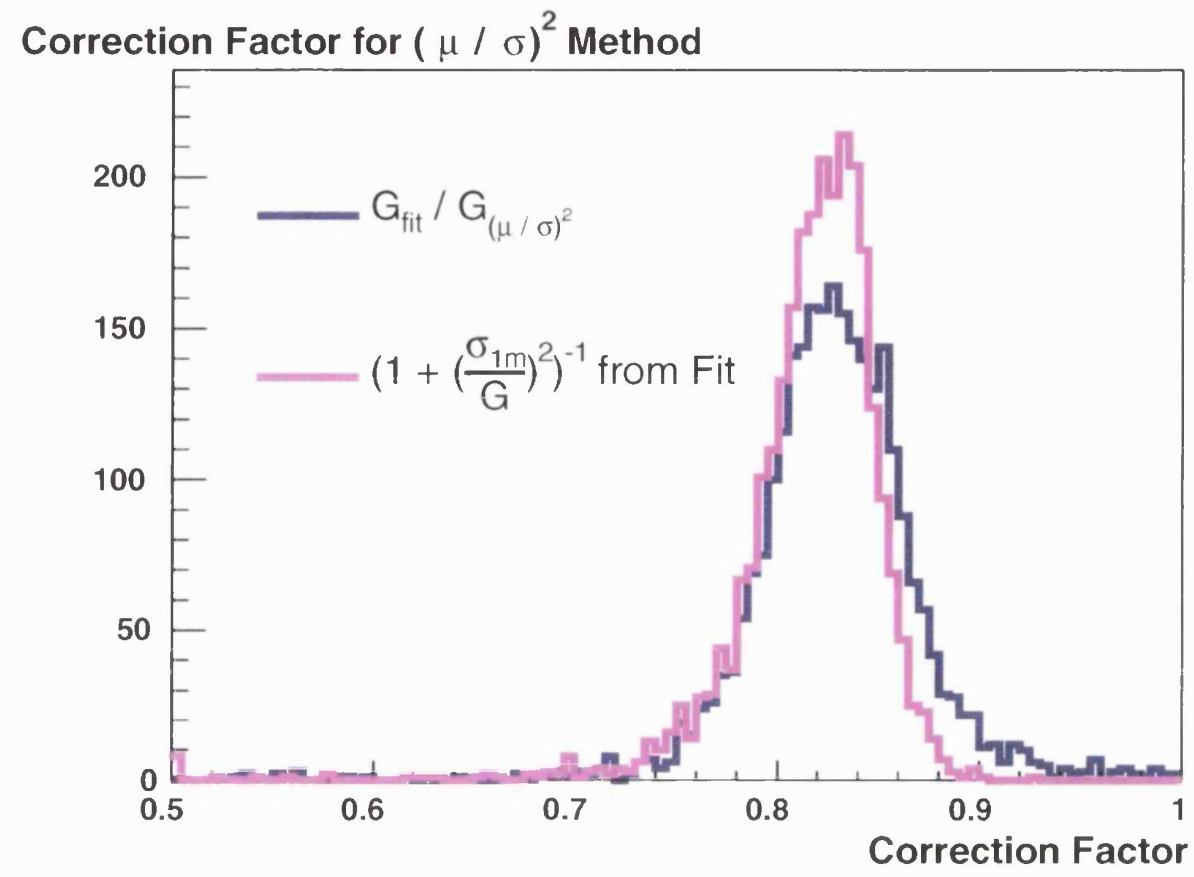

Figure 7.4: The ratio of the gain determined through the multi-Gaussian fit and the $(\mu / \sigma)^{2}$ and the expected value of this ratio from the width of the 1 PE peak are shown. The ratio is wider than predicted, largely due to measurement error and the inherent non-Gaussian shape of the $1 \mathrm{PE}$ peak.

in [65]. This simulation models the multiplication at each of the twelve dynode stages by a Poisson distribution at the previous dynode. At the photocathode the initial number of photoelectrons is modelled by a Poisson distribution with a mean of $\lambda$. A modification was added to allow a small probability that the first stage of dynode multiplication is skipped (i.e photons that pass through the photocathode), this is to better match the structure in the data between the pedestal and $1 \mathrm{PE}$ peak. An example of a simulated ADC spectrum is shown in Figure 7.5.

Using the simulation it is possible to test the statistical and systematic significance of the light level and number of pulses (i.e entries in the ADC spectra). Figure 7.6 shows the fractional deviation from the true value of the gain, and the RMS of the measured value of the gain for a mean light level of 1.0 PE. For each point in the plot 1000 spectra were generated, and the mean and RMS of the re- 


\section{Simulated ADC Spectrum}

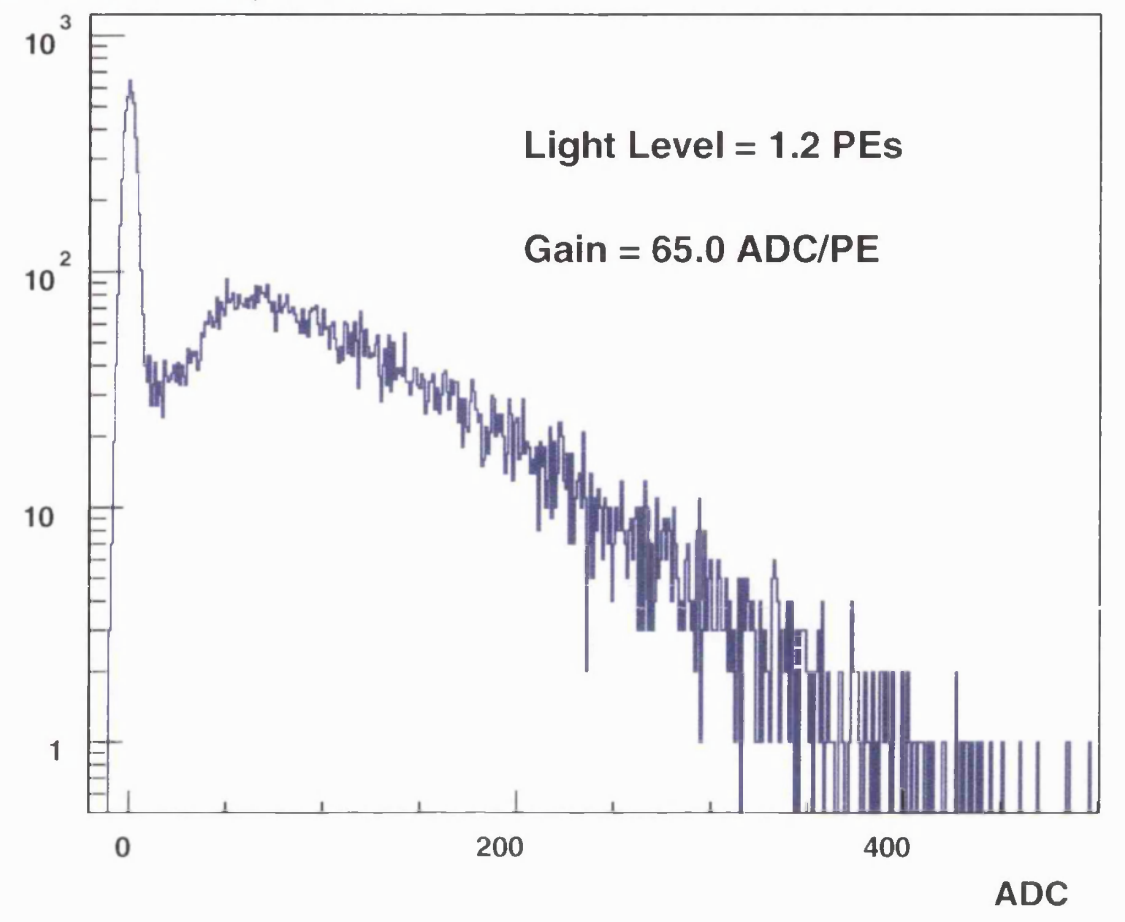

Figure 7.5: A simulated M16 ADC spectrum. The simulation models the multiplication at each dynode and the initial number of photoelectrons at the photocathode with Poisson distributions. In the simulation there is a small probability that the first stage of multiplication will be skipped, to account for photons that pass through the photocathode and produce electrons at the first dynode. This probability was added so that the simulation better matched the structure between the pedestal and 1 PE peak seen in the data.

sulting gain distributions were taken. The $(\mu / \sigma)^{2}$ gains are scaled such that the 100000 entries point matches the gain from the fit, this is to take into account the overestimate due to secondary emission at the first dynode. This scale factor is used in all of the comparison plots. After scaling, the $(\mu / \sigma)^{2}$ is the least biased of the four methods. Above 20000 entries the gain determined directly from the fit has little bias, but the value obtained using the light level from the fit has some systematic dependence on entries.

The gain determined using the number of pedestal entries shows a systematic difference from the true gain. This comes from overestimating the number of zeros 


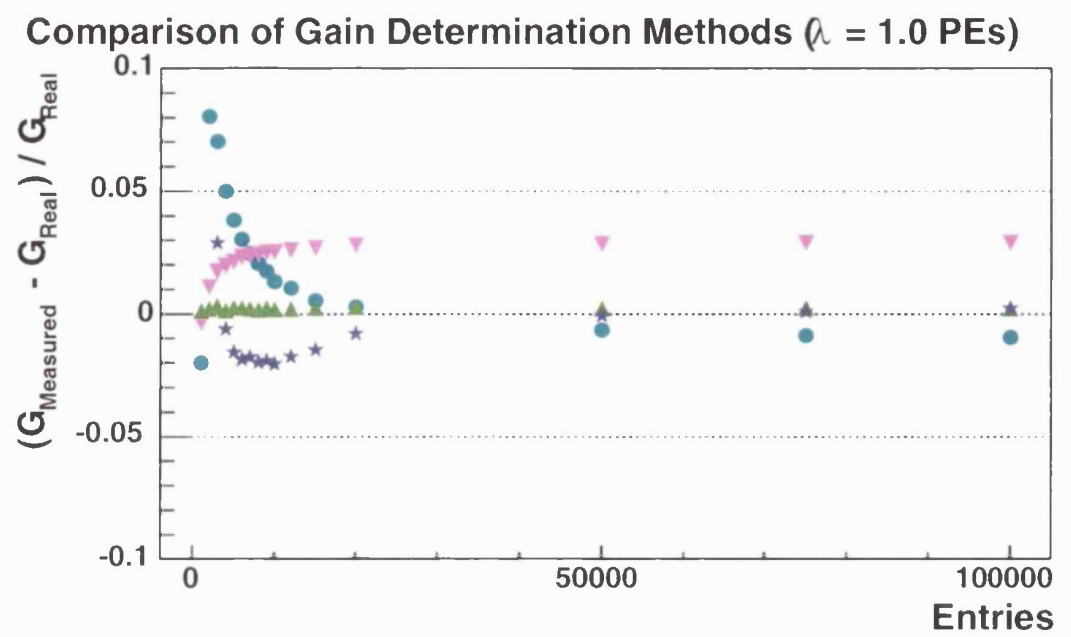

\section{RMS of Gain Distribution (from 1000 events)}

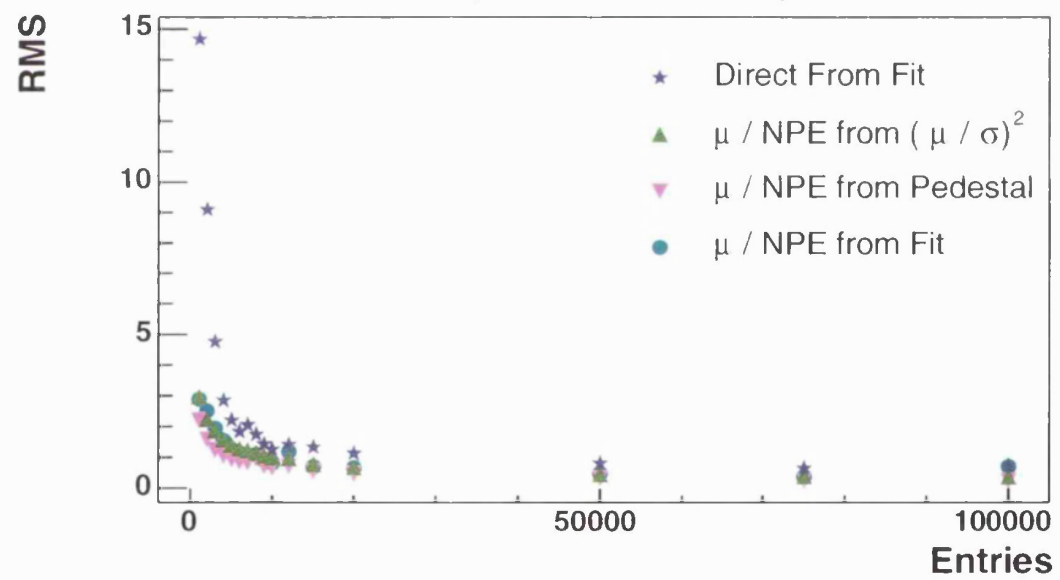

Figure 7.6: Performance of the four gain determination methods as a function of number of entries for simulated data. The top plot shows the fractional deviation from truth of the measured gain as a function of number of entries (i.e number of light injection pulses). At each point 1000 spectra were generated and the means of the resulting gain distributions were taken. The $(\mu / \sigma)^{2}$ method results uses a scale factor determined from comparison with the fitted gain, to take account of the broadening due to secondary emission at the first dynode. The bottom plot shows the RMS of the gain distributions, as an estimate of the error on a single measurement. 
using a Gaussian fit to the pedestal. The fit includes the low end tail of the single photoelectron peak and also those single photons which pass through the photocathode and produce an electron at the first dynode. Using the true number of zeros from the simulation, this over estimation becomes evident and the true gain value is recovered as shown in Figure 7.7.

The systematic dependence on light level of the gain determining methods has also been investigated using the simulation, see Figure 7.8. Once again the $(\mu / \sigma)^{2}$ method is the least biased of the four methods. The pedestal entries method varies only slightly with light level, but has a constant offset from the true value of the gain, as explained above. The two methods involving the fit are fairly flat between 1 - 3.5 PEs, with the direct method being the least biased of the two. At very low light levels, below $\sim 1 \mathrm{PE}$, the fit exhibits a systematic dependence on light level, this is due to the very small fraction of non-pedestal entries to which Gaussians are being fit. At higher light levels, above 3.5 PEs, the fit struggles due to a lack of entries in the pedestal, this is reflected in the increased RMS of the gain distribution at higher light levels.

The $(\mu / \sigma)^{2}$ method is clearly a powerful method for determining the gain of the PMT pixels. However, the greatest strength of the method is that it can be used at higher light levels where the pedestal is not visible (assuming the distribution is pedestal subtracted, as it is the MINOS electronics), see Figure 7.9. As before, 1000 spectra, containing 10000 entries, were generated at each light level. The means of the resulting gain distributions show no systematic dependence on light level, and vary by less than $0.25 \%$ over the range. The typical error on a single measurement of the gain, inferred from the RMS of the distribution, is $\sim 1 \mathrm{ADC}$ count.

At the CalDet the gain of the PMT pixels was initially determined using the multi-Gaussian fit. Using these values the $(\mu / \sigma)^{2}$ scale factor was determined. After this initial determination the gain has been determined with the scaled $(\mu / \sigma)^{2}$ method using higher light level data, which is more easily obtainable than the low light level data.

So far, one scale factor has been used for all of the pixels in the detector, this 

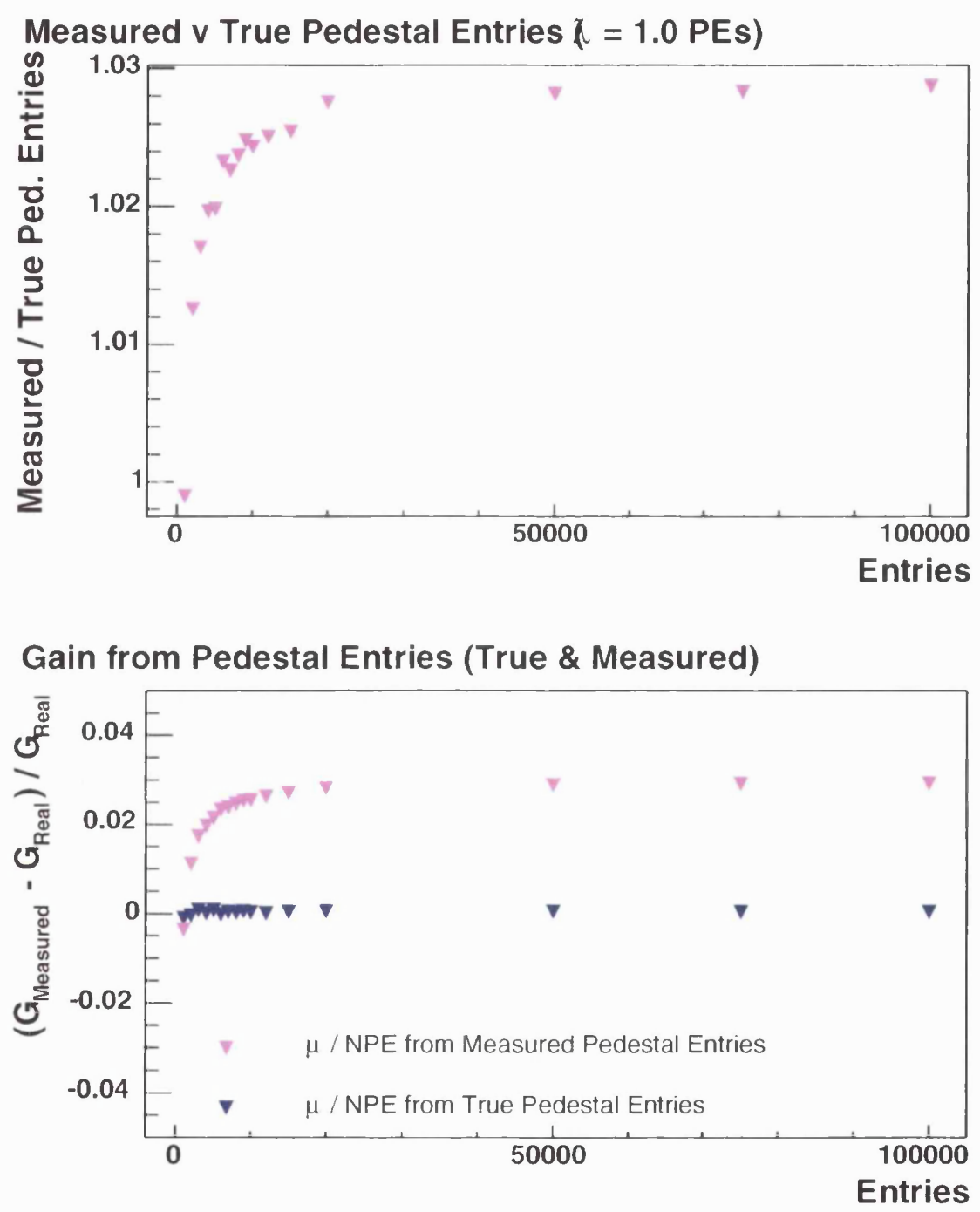

Figure 7.7: The upper plot shows the ratio of measured pedestal entries to the true number from the simulation. In the bottom plot the gains calculated using the measured and true pedestal entries are shown. Using a Gaussian fit to the pedestal, the number of zeros (and hence the gain) is overestimated. This overestimation is due to the inclusion of the low tail of the single photoelectron peak, and those single photons which pass through the photocathode and produce an electron at the first dynode. Using the true number of pedestal entries the correct value for the gain is recovered. 


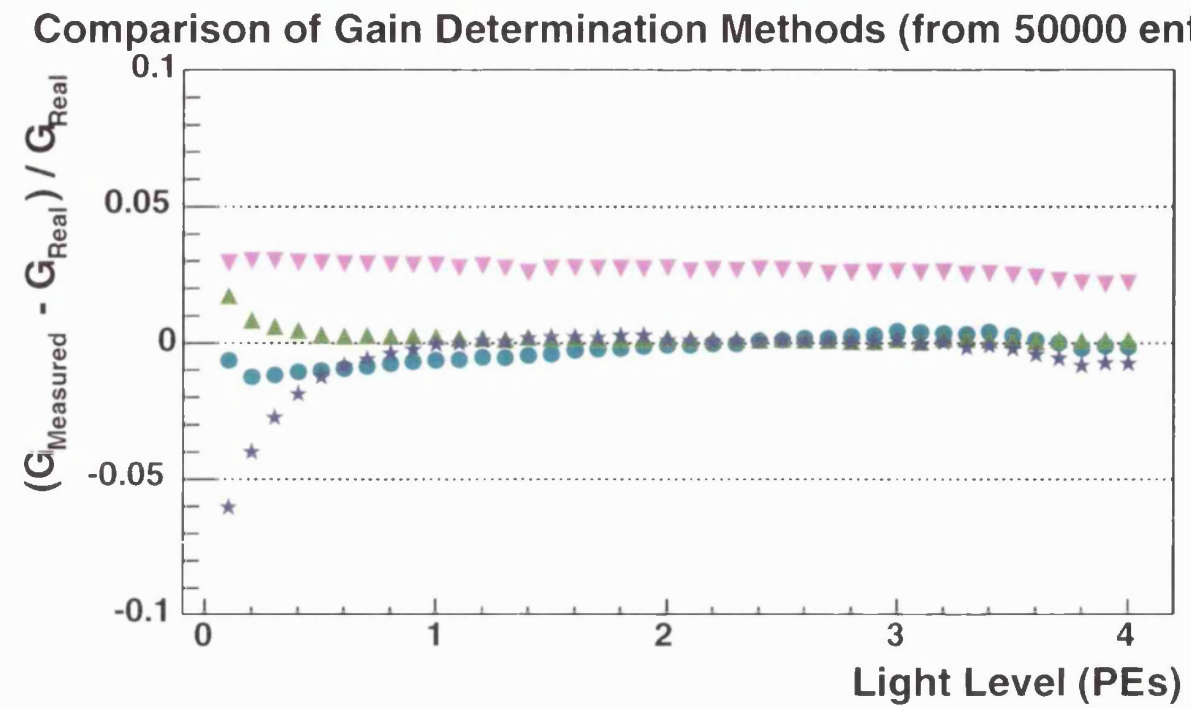

\section{RMS of Gain Distribution (from 1000 events)}

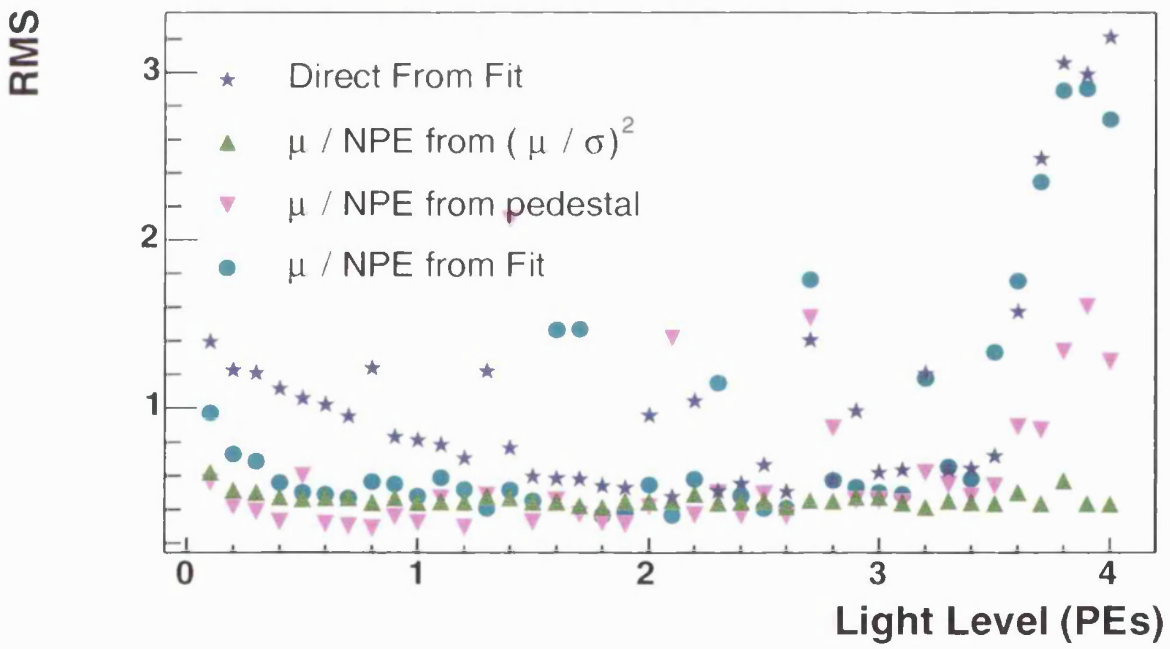

Figure 7.8: Performance of the four gain determination methods as a function of light level for simulated data. The top plot shows the fractional deviation from truth of the measured gain as a function of number of photoelectrons over the range 0.1 - 4.0 PEs. The bottom plot shows the RMS of the gain distributions, as an estimate of the error on a single measurement. The scaled $(\mu / \sigma)^{2}$ is the least biased of the methods, although the gain from the fit has very little bias between $1-3.5$ PEs. 
will add an extra uncertainty to the gain given by the width of the underlying distribution in Figure 7.4. To obtain a more accurate measurement of the gain the scale factors should be calculated on a pixel by pixel basis.
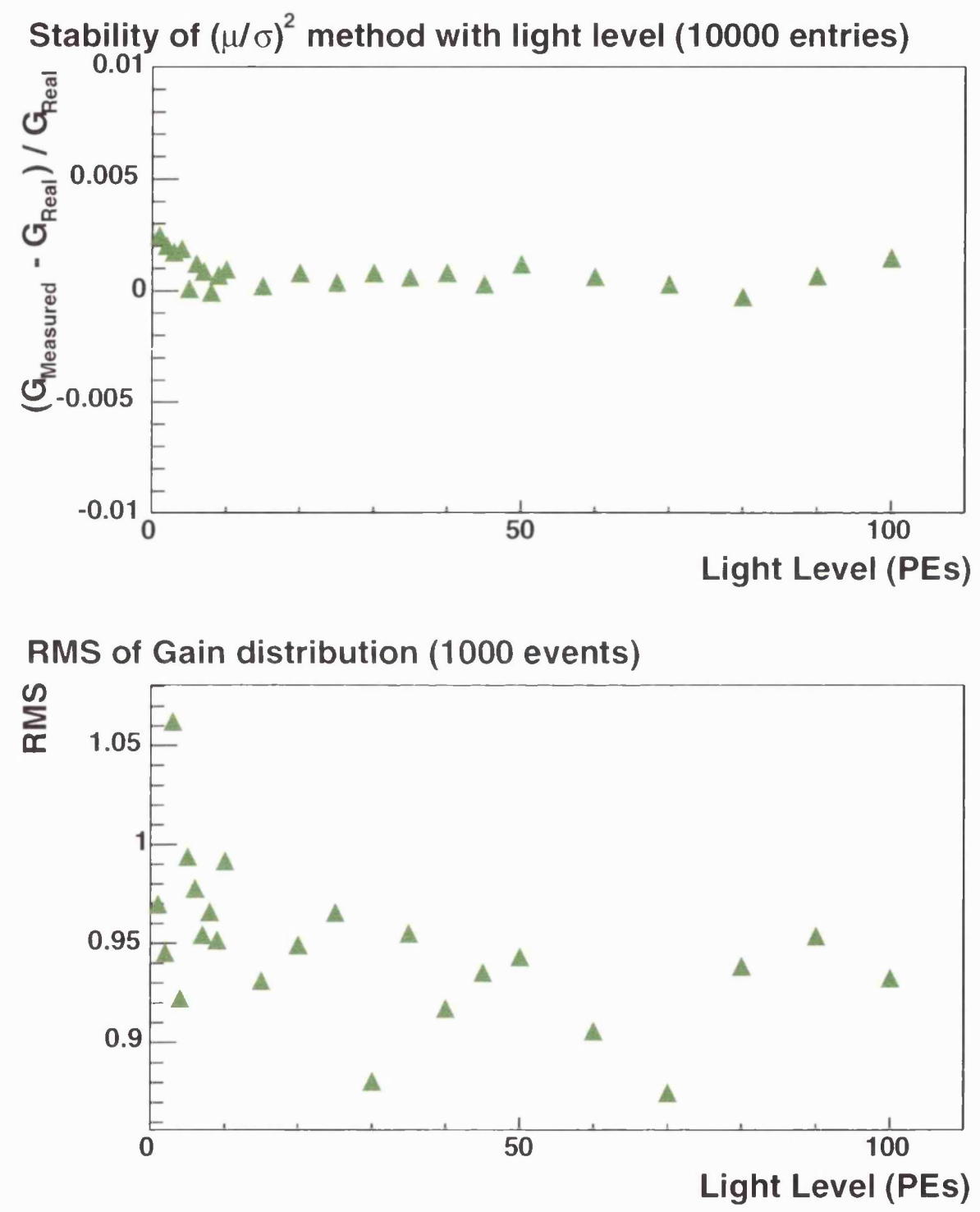

Figure 7.9: The stability of the $(\mu / \sigma)^{2}$ method of gain determination over light levels ranging from 1 - 100 PEs. The mean of 1000 measurements of the gain at each light level varies by less than $0.25 \%$ over the full range. The typical error on a single measurement, as implied by the RMS, is less than 1 ADC count for 10000 light pulses. Note that the scale of this plot is $\pm 1 \%$, compared to $\pm 10 \%$ in Figure 7.8 . 


\subsubsection{Linearity}

The light injection system is designed to inject light pulses over a large range of light levels. Using this capability it is possible to map out the linearity of the phototubes and electronics, by independently monitoring the amount of light injected with PIN diodes. In laboratory tests, these diodes have shown no signs of non-linearity [60].

The linearity of the phototubes is mapped out by pulsing the light injection system at different light levels, from $\sim 1-100$ PEs at the PMTs. Fibres travel directly from the pulser box to the PIN diodes, although the light which illuminates the PMTs is first absorbed by wavelength shifting fibre and re-emitted as green light, resulting in a factor of $\sim 1000$ more light at the PIN diodes than the PMTs. This is necessary as the PMTs are sensitive to much lower light levels than the PIN diodes. In the Far electronics configuration, the PIN diodes and PMTs are readout using the VA electronics. An example linearity curve for one of the channels in CalDet from this running period is shown in Figure 7.10.

Although the PIN diodes themselves have been shown to be linear, the VA electronics through which they are readout and digitized are known to be non-linear. Before the PIN diodes can be used to calibrate the non-linearity of the PMTs and their electronics, this non-linearity in the PIN electronics must be removed. This is done using the built in charge injection functionality of the electronics. A known amount of charge is injected at the input to the electronics, i.e. where charge from the PIN diode would be incident on the electronics, the response of the electronics to this charge can be determined 7.11.

Initially the pulser boxes were constructed using blue LEDs, rather than ultra violet LEDs. When linearity curves were taken at the CalDet by flashing these blue LEDs, they showed a non-linearity at low light levels in the region where the PMT and electronics had previously been shown to be linear. After exhaustive tests at the Calibration Detector, Far Detector and at university test stands, the cause of this non-linearity was traced to a slight shift in wavelength with applied voltage of the LEDs [68]. The shift in wavelength corresponded to a rapidly changing region of the absorption spectrum of the wavelength shifting fibre. This non-linearity is 


\section{Example Linearity Curve}

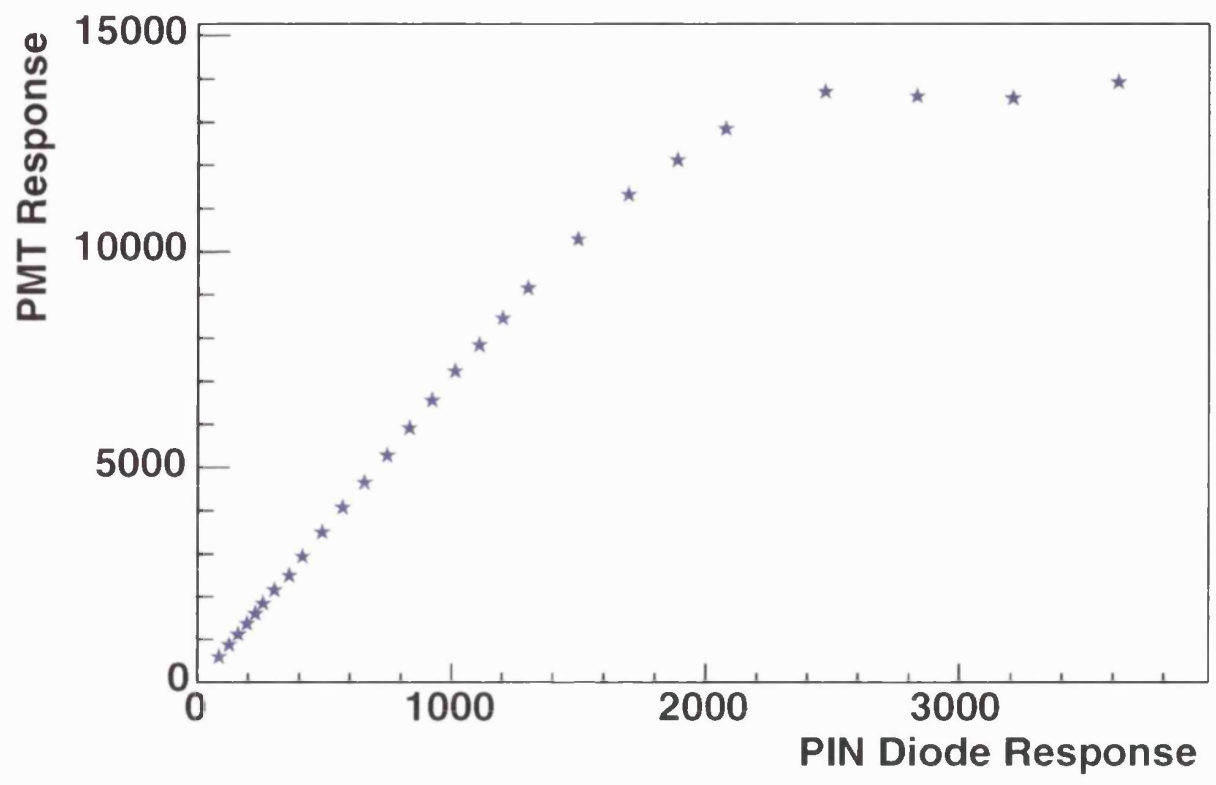

Figure 7.10: A light injection linearity curve from one of the CalDet channels. The VA electronics are known to become non-linear and saturate above $\sim 8000$ counts, as can be seen in the PMT scale of this channel.

illustrated in Figure 7.12, with a straight line fit to the lower portion of the curve.

The UV LEDs were chosen as they exhibit a smaller change in emitted wavelength with voltage, and the emitted wavelength lies on a much flatter region of the wavelength shifting fibre absorption spectrum [68]. Figure 7.13 is an example of a straight line fit to the PMT - PIN response curve showing the linearity at low light levels. Using these LEDs the distribution of residuals in the fitted region is very flat, and the light injection system can be used to linearize the response of the PMTs and electronics. 


\section{Example Charge Injection Curve}

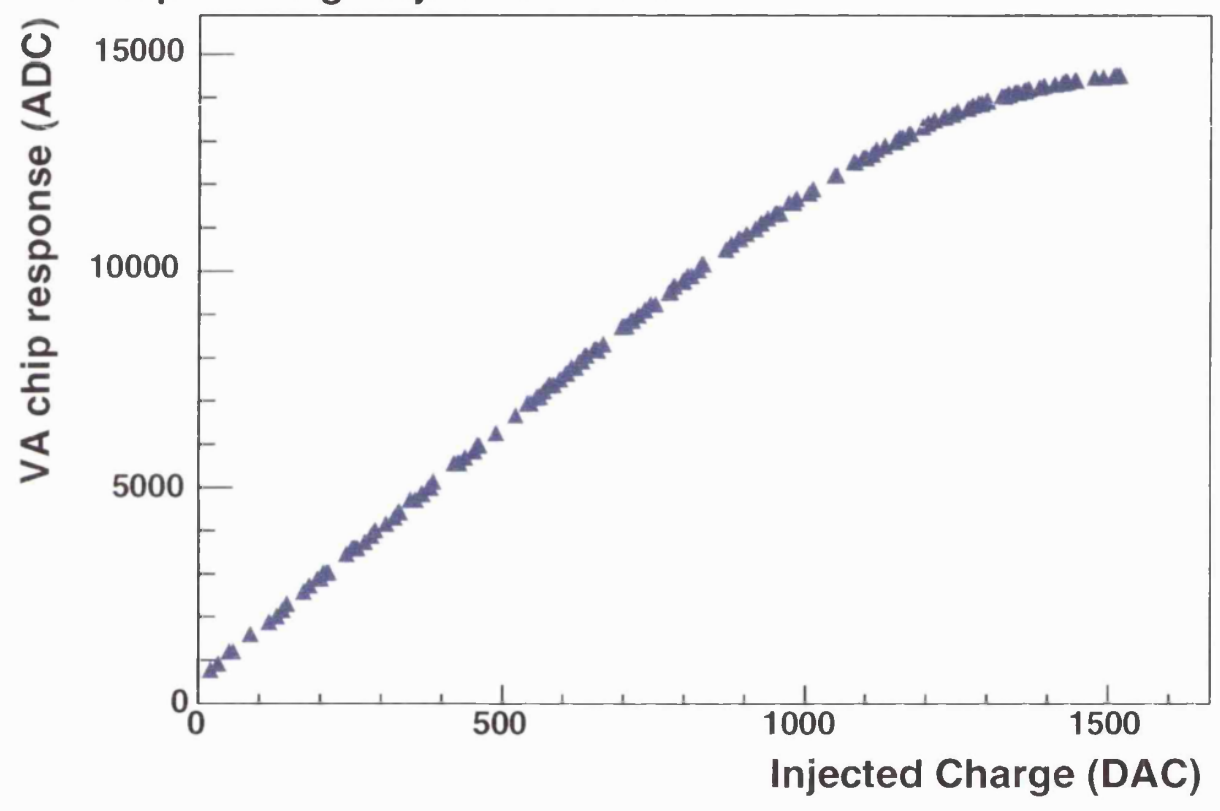

Figure 7.11: A charge injection curve from one of the PIN diode channels in the CalDet. A controlled quantity of charge can be injected at the input to the VA chip, the response of the electronics to this charge can then be determined. The PIN diode electronics must be linearized before the diodes can be used to calibrate the PMT channels. 


\section{Example Linearity Curve (with Blue LED)}

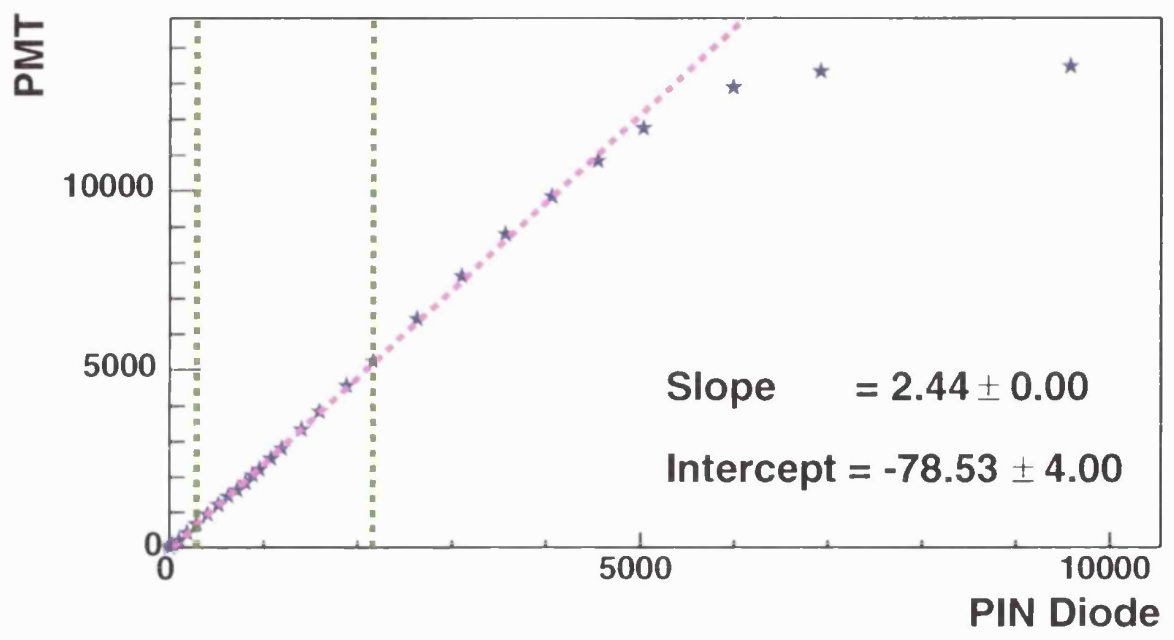

\section{Residuals to Straight Line Fit}

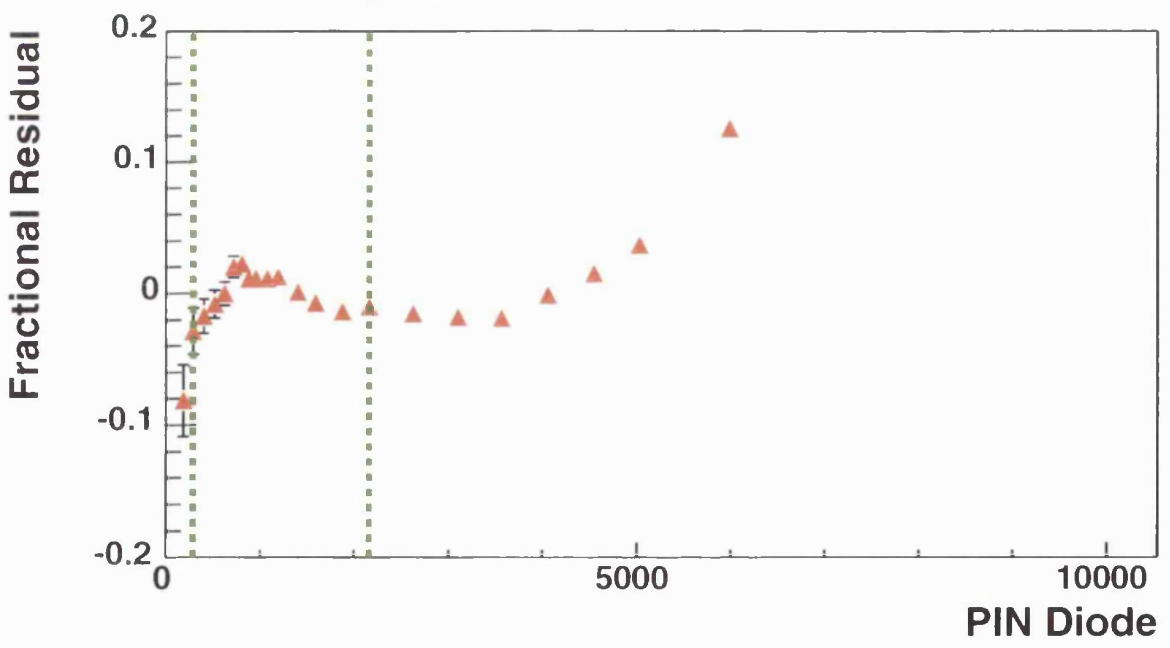

Figure 7.12: The non-linearity in PMT - PIN response observed when blue LEDs illuminate wavelength shifting fibre. Residuals are plotted to a straight line fit in the region marked by the green lines. This non-linearity was traced to a slight shift in wavelength emitted with applied voltage on the LEDs, coupled with a rapidly changing section of the wavelength shifting fibre absorption spectrum. 


\section{Example Linearity Curve (with UV LED)}
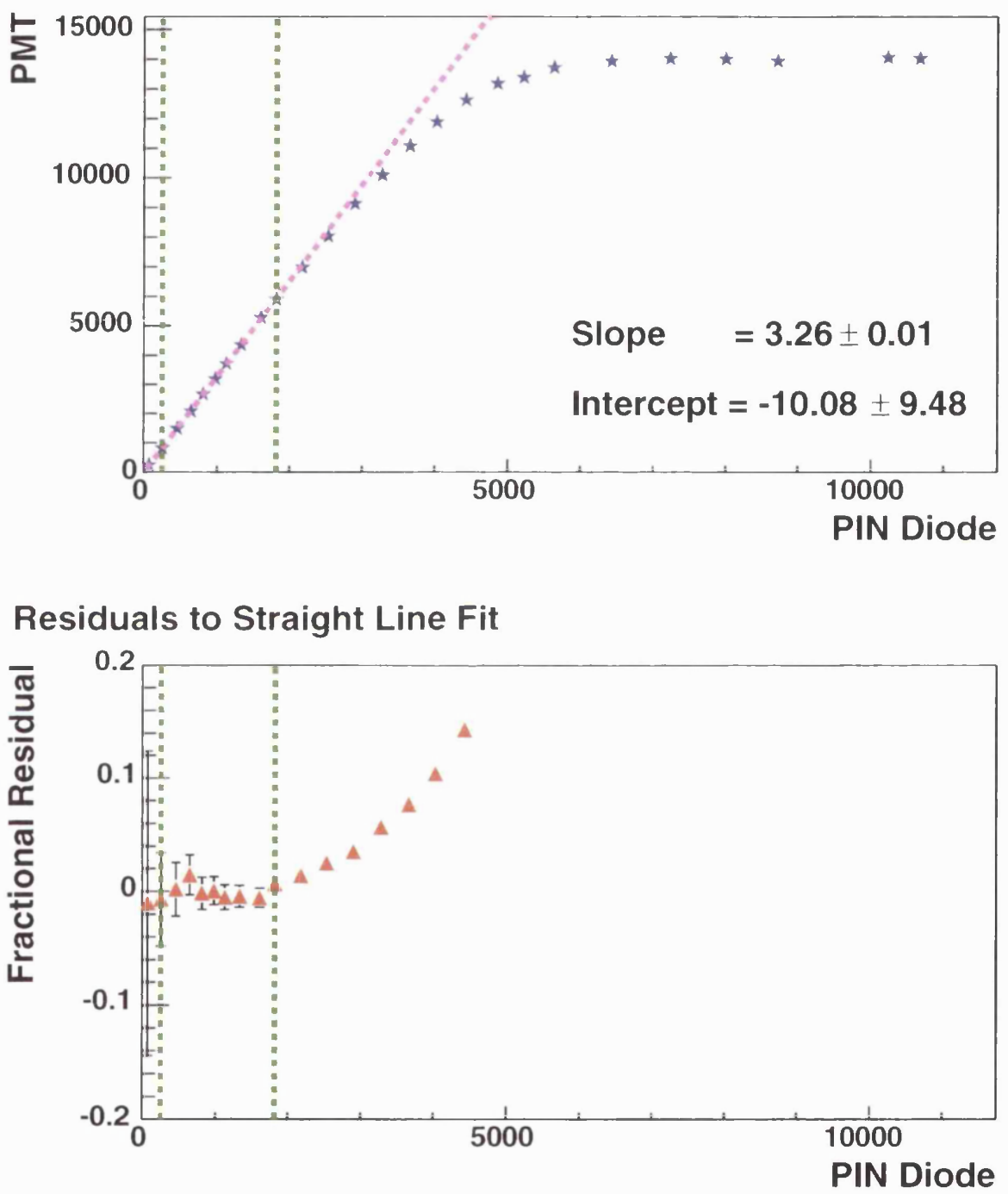

Figure 7.13: Using ultraviolet LEDs the PMT - PIN response is linear at low light levels. Residuals are plotted to a straight line fit in the region marked by the green lines. There is still the non-linearity at high light levels due to the PMT and electronics. 


\subsubsection{Drift Point Calibration}

Perhaps the most important function that the light injection system provides is the drift point calibration. This is important as it allows an intermediate calibration of the PMTs and electronics while muons are being collected for use in the strip to strip and cross-detector calibrations. In the Far Detector, due to the low muon flux ( $\sim 500$ per strip per month) it can take up to a month to collect enough muons for these calibrations. The light injection system was designed to remove drift in the response of the PMTs and electronics to the $1 \%$ level over the timescale of a month. This is necessary if the relative calibration using muons will be carried out at the $2 \%$ level. At the CalDet the muon flux is much higher, as it is on the surface and not in a mine, and sufficient statistics to calibrate the detector can be accumulated in $\sim 1$ hour. This means that a drift point correction is not strictly necessary, however one of the functions of the CalDet was to test the proposed calibration procedures.

The drift point calibration consists of flashing the LEDs at a single height, and monitoring the response of the PMTs using the PIN diodes to remove any variation in the response of the LEDs. The Calibration Detector resides in a much noisier location than the Far Detector, fluctuations in temperature and background radiation are much larger in the testbeam hall than in the Soudan mine. This coupled with the fact that there are only 6 LEDs in CalDet, compared to 320 in the Far Detector, meant that it was both desirable and possible to take a drift point with 2500 flashes every 20 minutes. An example of the PMT response to these drift points, over a 6 day period, is shown in Figure 7.14. Before correction the drift is on the order of a few percent, mainly due to temperature effects.

The drift point corrections are used to monitor the short term (up to a month) response of the phototubes and electronics. In order to accurately track changes in the response, the means of the drift point ADC distributions must be well known. The optimum number of light injection flashes used for each drift point has been investigated, the fractional error on the mean (assuming Gaussian errors) is plotted as a function of mean for four different number of flashes in Figure 7.15. To ensure the means of the drift point distribution are known to better than $0.5 \%$ over the 


\section{Drift Point Response of Single PMT Channel (Over 6 Days)}

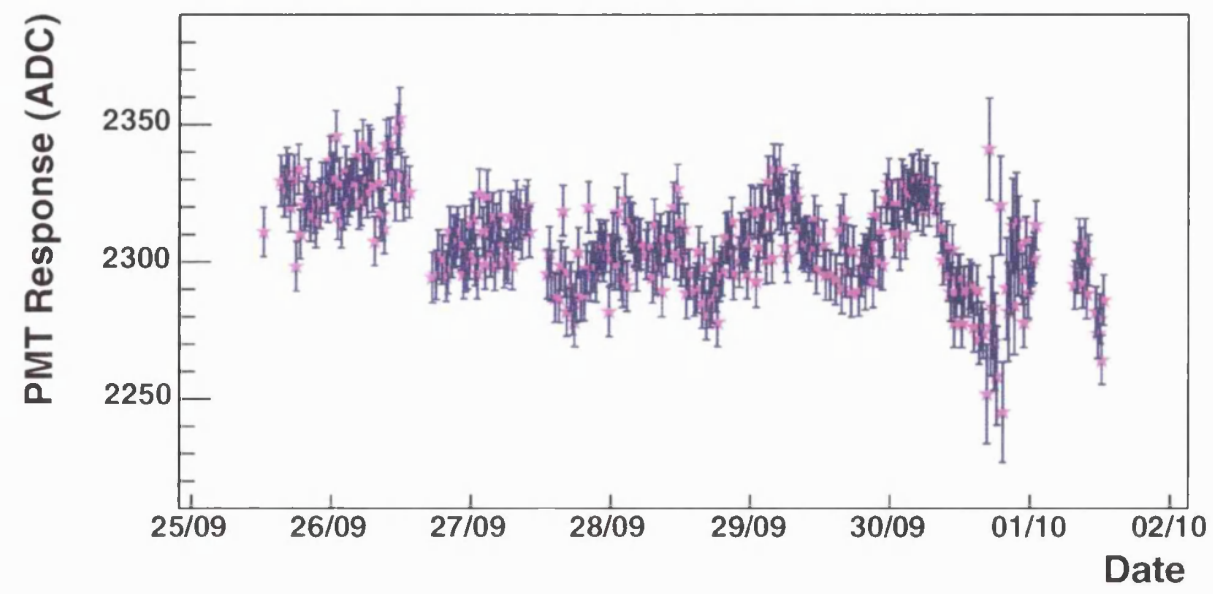

Figure 7.14: The response of a single PMT channel is plotted against time over a 6 day period. Each point is formed from 2500 LED flashes. The oscillatory shape is due to variations in temperature.

full linear dynamic range, 2500 flashes were chosen for the CalDet drift points. The downside of using such a large number of flashes per drift point is that the data takes longer to accumulate. At the Far Detector it takes over an hour to flash each of the 320 LEDs 1000 times. Using 1000 flashes still measures the mean of the drift point distribution to better than $1 \%$.

The drift points are used to construct calibration constants using the response of the PIN diodes to monitor the light level produced by the LED. In the linear regime the correction used, $C_{\text {Drift }}(t)$, at time, $t$, is the ratio of ratios:

$$
C_{\text {Drift }}(t)=\frac{\mu_{P M T}(t) / \mu_{P I N}(t)}{\mu_{P M T}(0) / \mu_{P I N}(0)},
$$

where $\mu_{P M T}(t)$ and $\mu_{P I N}(t)$ are the mean of the PMT and PIN drift point distributions at time $t$.

At the Calibration Detector the major cause of gain drift is temperature fluctuations, both the PMTs and electronics are known to be sensitive to changes in temperature. This can be evidenced by comparing the average drift point correction and temperature as functions of time which is plotted over a six day period in Figure 7.16. The average correction shows less than $1 \%$ coherent drift in the gain of 


\section{Fractional Error on Mean}

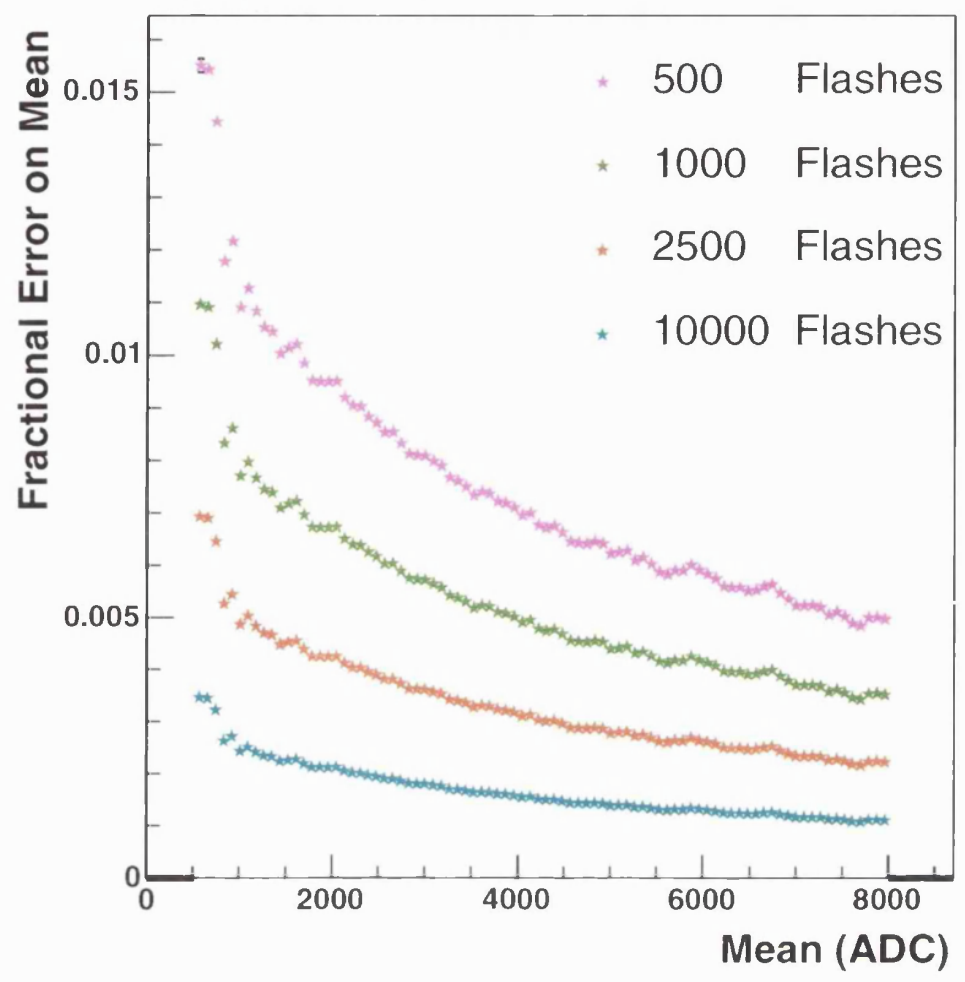

Figure 7.15: The fractional error on the mean of the drift point ADC distribution, assuming Gaussian errors, is plotted against mean. Four different values for the number of flashes in each drift point are plotted. So that the means are known to better than $0.5 \%$ across almost the full linear dynamic range, 2500 flashes are used at the CalDet.

the channels, and there is a clear inverse correlation between the temperature and the drift.

The distribution of drift point corrections shows that the majority of channels exhibit little drift over the six day period, as in Figure 7.14, but there are a small number of anomalous channels which exhibit a larger drift in gain, see Figure 7.17. The central peak has an RMS of $1 \%$ and corresponds to almost $98 \%$ of the channels in the detector. The remaining channels have rapidly changing gain, which is usually symptomatic of a serious malfunction in either the PMTs or electronics. The drift with time of one such malfunctioning channel is shown in Figure 7.18. The gain in 


\section{Temperature (Over 6 Days)}

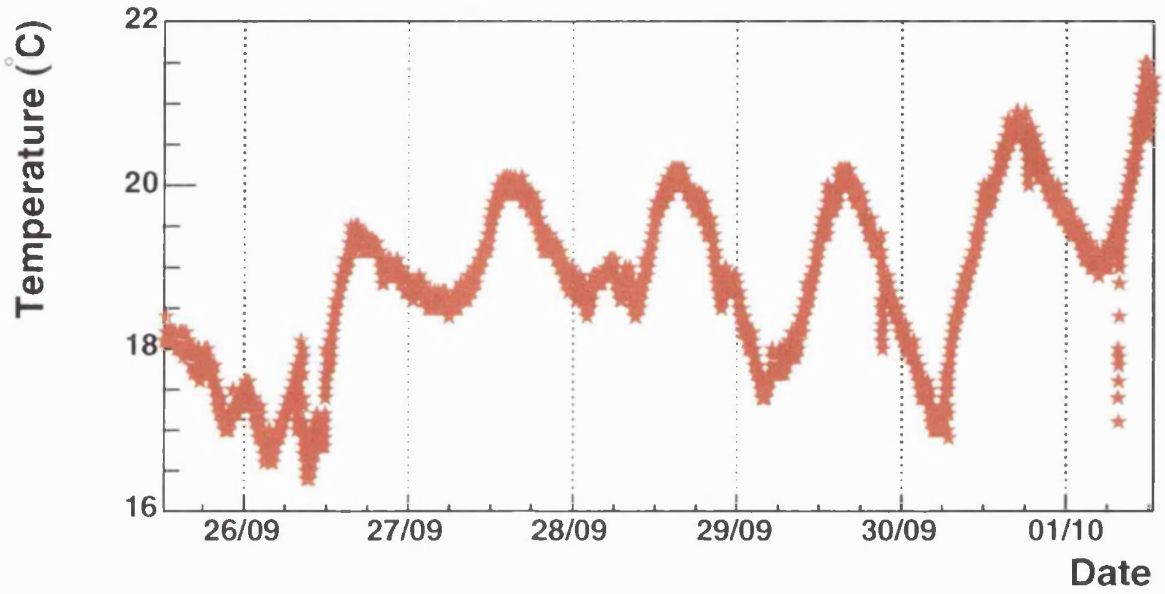

Average Drift Point Correction (Over 6 Days)

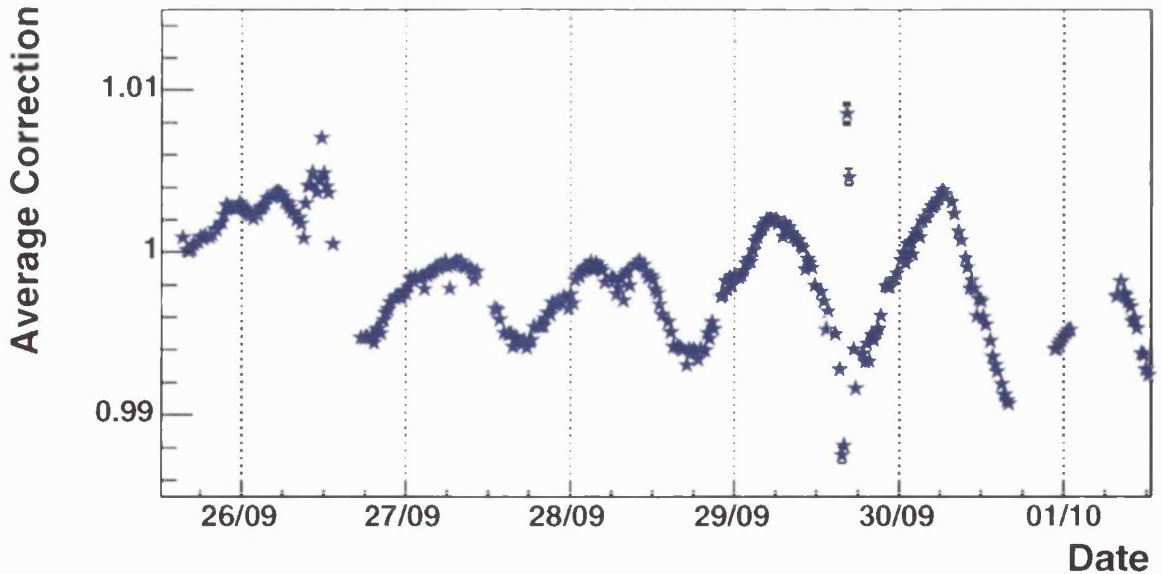

Figure 7.16: In the bottom plot the average gain drift correction is plotted as a function of time for a 6 day. The top plot shows the temperature fluctuations over the same time period. There is a clear inverse correlation between the drift in gain and the changes in temperature. 
this channel changes by factor of 4 from lowest to highest during the six day period. This plot illustrates one method by which the light injection system can be used to debug hardware problems in the detector.

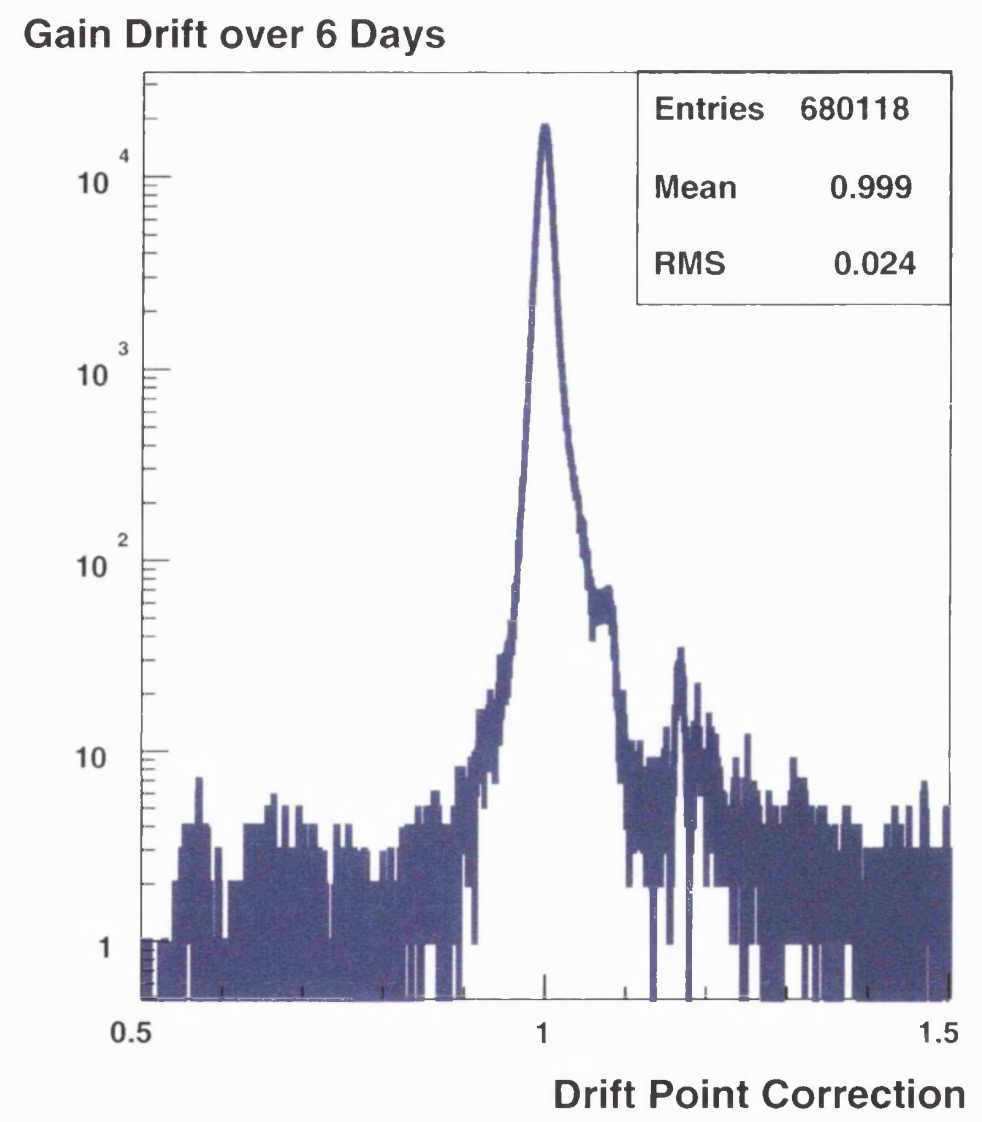

Figure 7.17: Histogram of the drift point corrections over the six day period shown in Figure 7.16. The central peak has an RMS of $1 \%$ and corresponds to $98 \%$ of the channels in the detector. The remaining channels all exhibit some form of pathological behaviour stemming from malfunctioning PMTs or electronics.

\section{Drift Point Calibration to $1 \%$}

The light injection system was designed to calibrate gain drift to the $1 \%$ level. In order to show that the system is capable of this, linearity curves have been employed. One point on the linearity curve is taken to be the drift point and is used to calibrate 
Drift Point Response of Malfunctioning Channel (Over 6 Days)

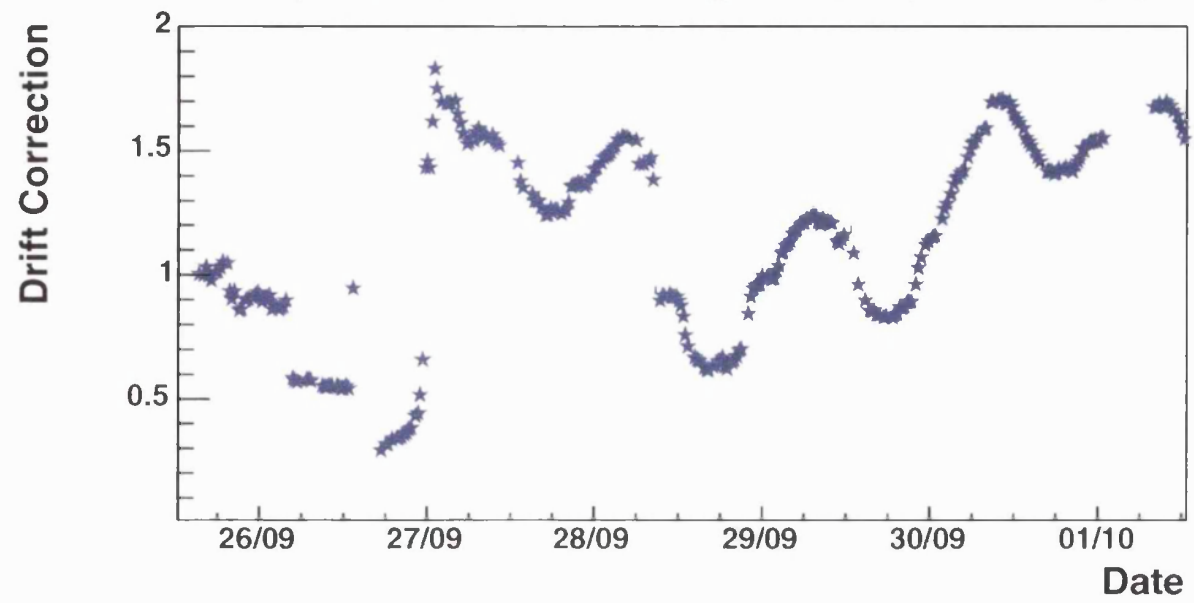

Figure 7.18: The response of a single malfunctioning channel is plotted against time over a 6 day period. There is a factor of 4 between the lowest and highest gain of the channel. This abnormally large drift in gain was due to faulty electronics, which were replaced shortly after the time period shown.

out any gain changes between successive linearity curves. This is done by evolving the previous gain curve, such that the corrected PMT - PIN response curve reflects any changes in the gain of the phototubes. An example of this evolution is shown in Figure 7.19. The large change in gain between the two data sets was the result of an increase in the high voltage applied to the PMTs.

A second point on the linearity curve is taken as the test point. By comparing the mean of the ADC distribution at this point to that predicted from the previous gain curve, before and after drift correction, the performance of the light injection system can be evaluated. Any fluctuations in LED light output are removed using the PIN diodes:

$$
\begin{gathered}
\mu_{\text {uncorrected }}=\mu(0)_{P M T} * \frac{\mu(t)_{P I N}}{\mu(0)_{P I N}} \\
\mu_{\text {corrected }}=\mu^{\prime}(0)_{P M T} * \frac{\mu(t)_{P I N}}{\mu^{\prime}(0)_{P I N}},
\end{gathered}
$$

where $\mu(0)$ refers to the test point on the original unaltered linearity curve, $\mu(t)$ to the test point on the latest linearity curve and $\mu^{\prime}(0)$ to the test point from the 


\section{Linearity Curve Evolution with Drift Point}

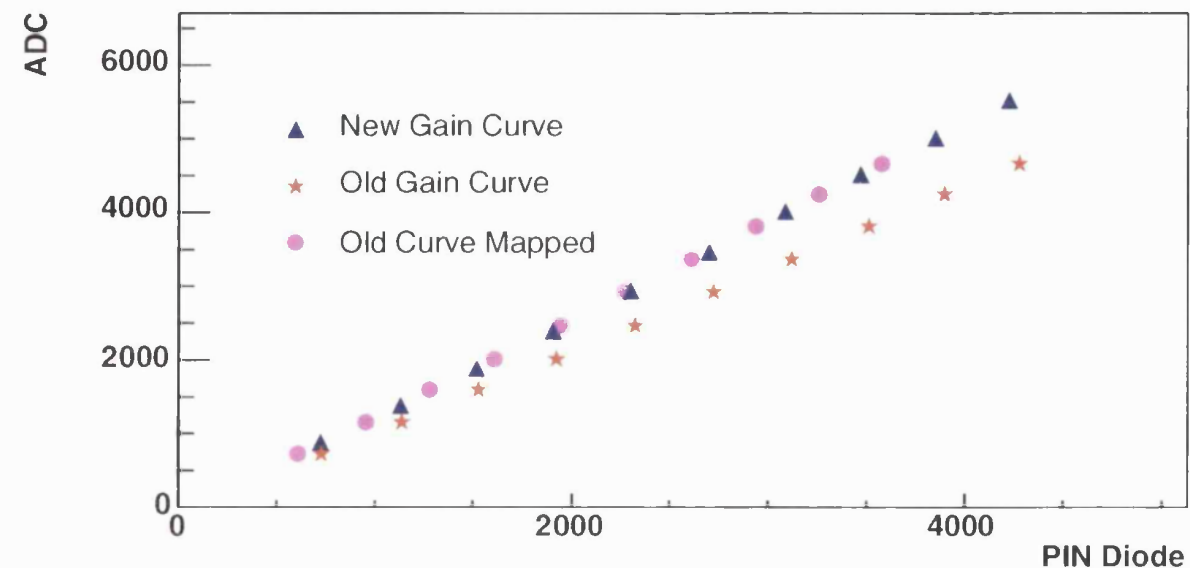

Figure 7.19: Two PMT - PIN linearity curves from a single channel are shown. The two curves were separated by two weeks and a $20 \%$ increase in PMT gain due to raising the high voltage on the phototube. Using a single point on the curve as the drift point, it is possible to evolve the linearity curve to account for this gain change.

evolved original linearity curve. The fractional difference between the predicted values of test points and the measured values before and after drift correction are shown in Figure 7.20. In the upper and lower histograms the plotted quantities are

$$
\begin{array}{lr}
\text { Upper : } & \left(\mu_{\text {uncorrected }}-\mu(t)\right) / \mu_{\text {uncorrected }} \\
\text { Lower : } & \left(\mu_{\text {corrected }}-\mu(t)\right) / \mu_{\text {corrected }}
\end{array}
$$

The uncorrected distribution shows the intentionally induced $20 \%$ increase in phototube gain, due to the increased high voltage between the first and second linearity curves. There was only a slight gain change between the second and third linearity curves, which is consistent with fluctuations in temperature in the testbeam hall. The corrected distribution is centred close to zero with a width of less than $1 \%$. 


\section{Before Drift Correction}

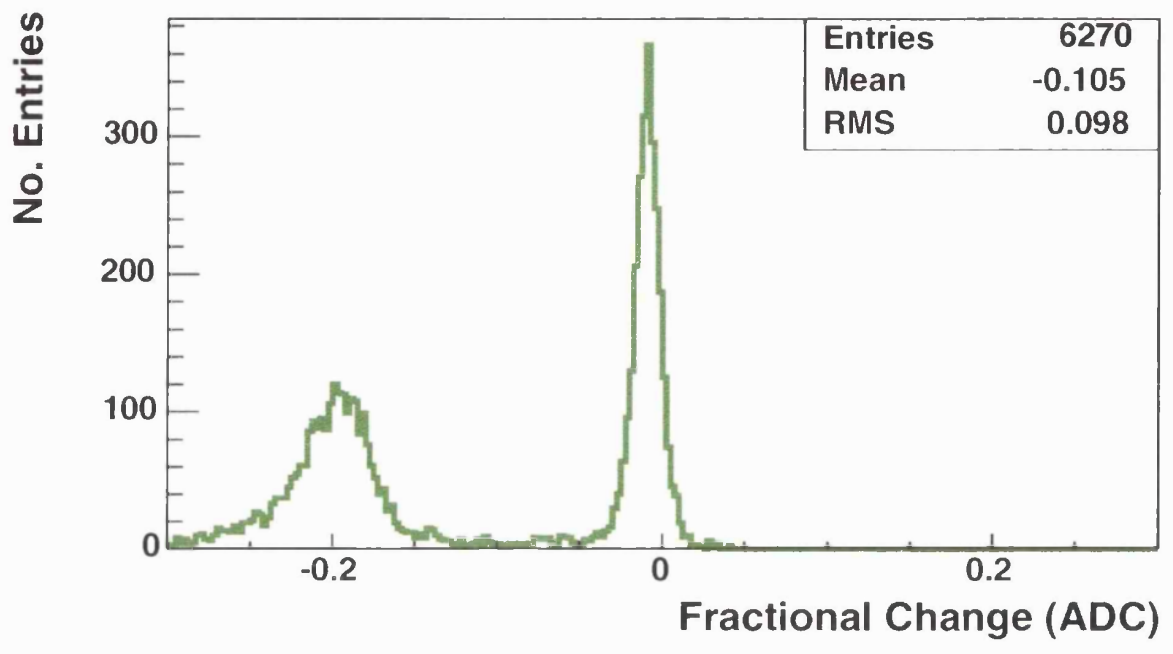

After Drift Correction

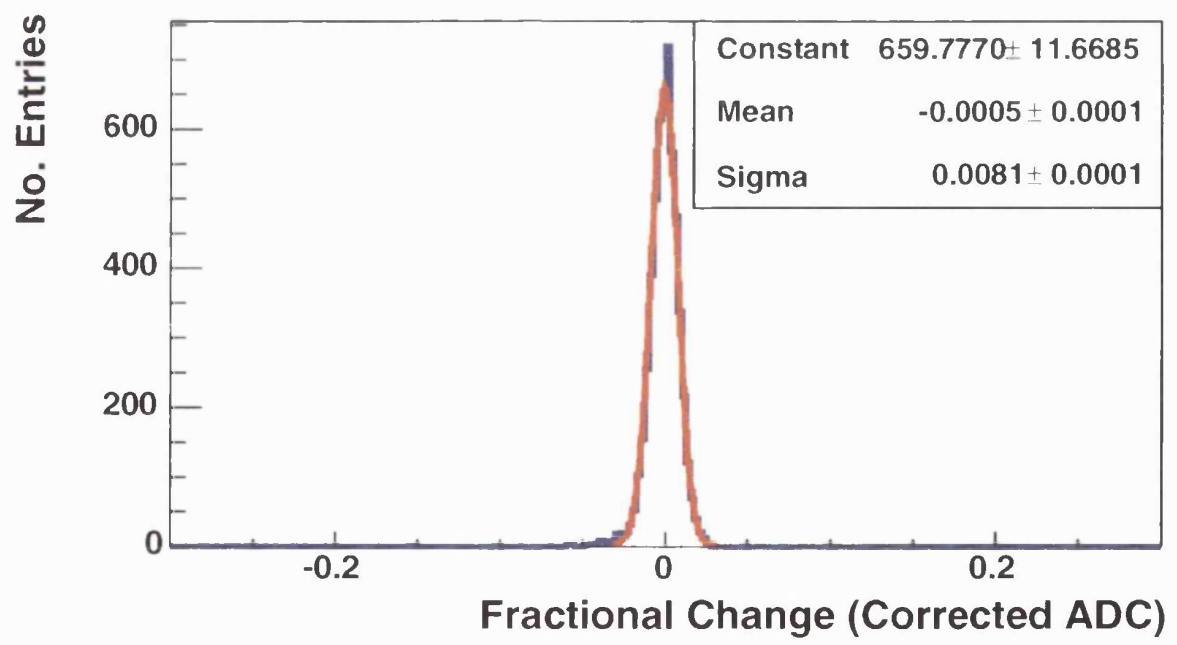

Figure 7.20: The upper distribution shows the fractional difference in PMT response between test points on three successive linearity curves. The $20 \%$ change in gain of the PMTs between two of the curves was due to a system wide increase in high voltage on the PMTs. The bottom plot shows the same distribution after drift point correction. The changes in gain are calibrated to the $1 \%$ level. 


\subsection{Strip to Strip Calibration}

A strip to strip calibration is necessary to ensure that the response of each strip in the detector is the same to particles passing through the strips. The light injection system can be used to remove variation in the response of the PMTs and electronics. However, there are several factors that can not be accounted for using the light injection system, these include:

- Scintillator light yield.

- Defects in wavelength shifting fibre.

- Optical connections and readout cables.

To fully characterize the response of a scintillator strip, energy deposits from charged particles traversing the strip must be used. The particles used for this calibration are muons, as they are the most readily abundant particle at the three MINOS detectors (from cosmic rays) and have several useful characteristics. One of the main advantages to using muons is that they are minimum ionizing particles, meaning that they deposit a similar amount of energy in each plane of scintillator they traverse.

At the CalDet there are three sources of muons: cosmic ray muons, beam muons and PS muons. The PS muons are muons produced from interactions, involving protons from the PS ring, close to the ring resulting in high energy muons that do not travel down the beam pipe. The beam muons are not suitable for a full calibration, as they do not illuminate all the strips in the detector.

Muon ADC spectra are used to characterize the response of strips in the detector. These spectra are formed using the energy deposits, in ADC, of muons passing through each scintillator strip. An example ADC spectrum from cosmic ray muons is shown in Figure 7.21. The asymmetric shape of the distribution is due to the underlying Landau nature of muon energy deposits and this underlying distribution is convolved with a Poisson distribution due to the photoelectron statistics at the PMT photocathode. Various methods for characterizing these spectra are discussed in [56] and providing there are enough statistics the mean of the spectrum is an 
adequate estimator of the characteristic light output of the strip.

A commonly used tool for reducing the statistical error on the mean, due to the large RMS from the tail, is to take the mean of a truncated distribution. The truncated distribution is formed by only including those entries below a threshold, the threshold is typically set at the 80-99th percentile. Although the statistical error on this mean is decreased, a systematic dependence is introduced as the Landau width varies with the width of material in which the energy is deposited. The truncation can then introduce a bias that depends on the angular distribution of muons used when forming the muon ADC spectra [69]. To avoid this systematic uncertainty the means of the muon ADC spectra are taken as the calibration constants.

\section{Typical Muon ADC Spectrum}

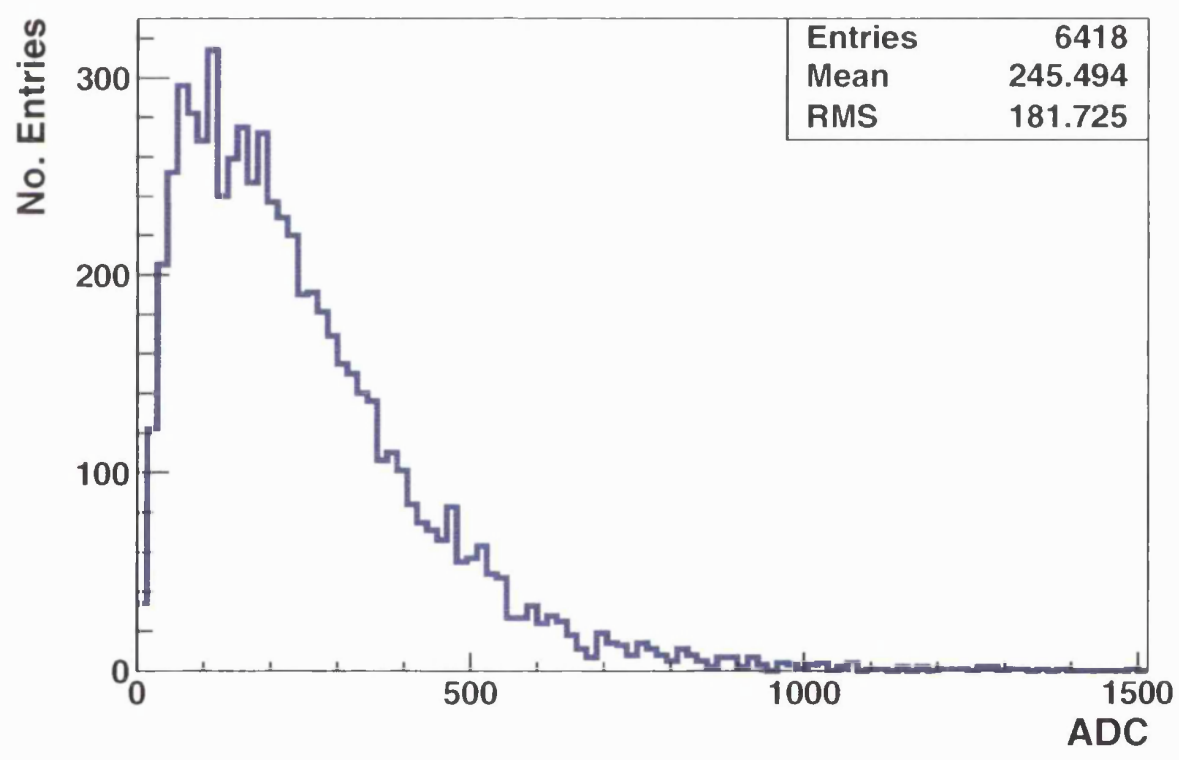

Figure 7.21: An example muon ADC spectrum. The long tail of the spectrum is due to the underlying Landau nature of muon energy deposits, this is convolved with a Poisson distribution due to the photoelectron statistics at the PMT photocathode.

There are several aspects of the detector response which must be taken into account when performing a strip to strip calibration using muons, including:

- Path length through each scintillator strip. 
- Energy of the muon.

- Attenuation in the scintillator module.

The relative importance of these factors depends on the choice of muon sample. Both cosmic ray muons and PS muons have been used to perform a calibration and the challenges of using these two muon samples are discussed in the following sections.

\subsubsection{Cosmic Ray Muons}

Cosmic ray muons are the logical choice of muon sample to use for calibrating strips in the CalDet, as both the Near and Far Detectors can be calibrated using cosmic ray muons.

\section{Straight Muon Tracking Algorithm}

The CalDet is situated on the surface of the Earth which leads to a cosmic ray spectrum with an average energy of $\sim 4 \mathrm{GeV}$. This, coupled with the angular distribution of the muons, results in the vast majority of muons passing through the detector and leaving short straight tracks.

The tracks are straight with very little multiple scattering, so they should, in theory, be easy to track. However, crosstalk in the PMT causes problems for tracking. PMT crosstalk is light, or charge, from one PMT pixel leaking into another pixel on the PMT, see Figure 7.22. The most common form of crosstalk is optical crosstalk to an adjacent pixel. As each pixel in the PMT is connected to a different strip in the detector, these crosstalk hits look like energy deposits from strips that the muon did not pass through. An example of this is shown in the cosmic ray event display in Figure 7.23, the strips highlighted in purple are prospective crosstalk hits. These hits must either be removed before tracking, or the muon tracker must be robust enough not to include these strips in the track.

To tackle the problem of confusion due to crosstalk along the track, a tracking algorithm was developed such that it would not include these spurious strips. The algorithm adopts an iterative approach to track finding, using the following steps: 


\section{Pixel Numbering}

\begin{tabular}{|c|c|c|c|}
\hline 4 & 3 & 2 & 1 \\
\hline 8 & 7 & 6 & 5 \\
\hline 12 & 13 & 10 & 9 \\
\hline 16 & 15 & 14 & 13 \\
\hline
\end{tabular}

Strip Numbering

\begin{tabular}{|c|c|c|c|}
\hline 14 & 2 & 13 & 1 \\
\hline 4 & 16 & 3 & 15 \\
\hline 18 & 6 & 17 & 5 \\
\hline 8 & 20 & 7 & 19 \\
\hline
\end{tabular}

Figure 7.22: An illustration of PMT crosstalk. When one pixel on a PMT is illuminated there is some chance that some of the light, or charge after multiplication, will leak into one or more of the other channels. In the above iliustration the picture on the lefthand side shows a single real hit, on pixel 6 , creating three additional crosstalk hits. The most common form of crosstalk is optical crosstalk to the neighbouring pixels, as with pixels 7 and 9 above, although charge crosstalk to a non-neighbouring pixel is possible, as with pixel 16 above. The righthand side shows how these pixels are connected to scintillator strips in the plane, the real hit in strip 3 creates 'fake' hits in strips 5, 8, and 16 which the muon did not pass through. These additional strip hits make tracking the path of the muon through the detector more difficult.

i) Divide the event into sections containing contiguous hit planes, using a gap of two planes or greater to separate the sections.

ii) Only attempt to track the longest of these sections.

iii) For each plane orientation fit a PE weighted straight line in plane-strip space.

iv) Discard any hit strips that are a distance greater than $\epsilon$ from the fitted line.

v) Repeat steps iii) and iv) until no more strips are discarded, and the track fit converges.

vi) Look at all the hit strips in the event and include those strips which are within $\epsilon$ of the fitted line.

vii) Check that the two track portions overlap and the three-dimensional reconstructed position of each hit is within the bounds of the detector.

The charge in the crosstalk channel is usually smaller than charge in the source channel. Nevertheless, in the case of optical crosstalk from a low light level source 


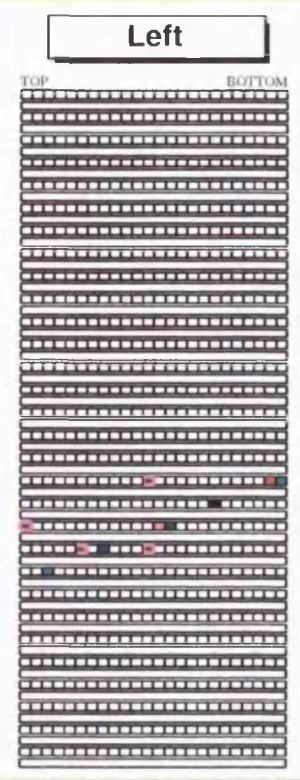

\begin{tabular}{|c|}
\hline Right \\
\hline 100 \\
\hline 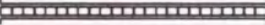 \\
\hline क्ञाता \\
\hline | \\
\hline 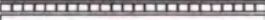 \\
\hline 年 \\
\hline 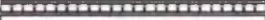 \\
\hline 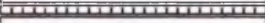 \\
\hline 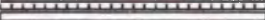 \\
\hline (101010 \\
\hline 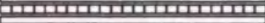 \\
\hline 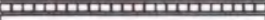 \\
\hline 80 \\
\hline 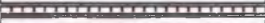 \\
\hline 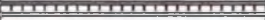 \\
\hline (101010 \\
\hline 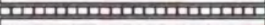 \\
\hline 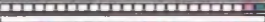 \\
\hline 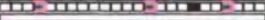 \\
\hline 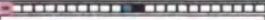 \\
\hline (II) \\
\hline 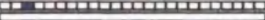 \\
\hline 监 \\
\hline \\
\hline 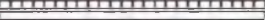 \\
\hline 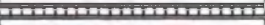 \\
\hline 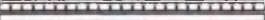 \\
\hline 年 \\
\hline 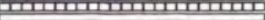 \\
\hline \\
\hline
\end{tabular}

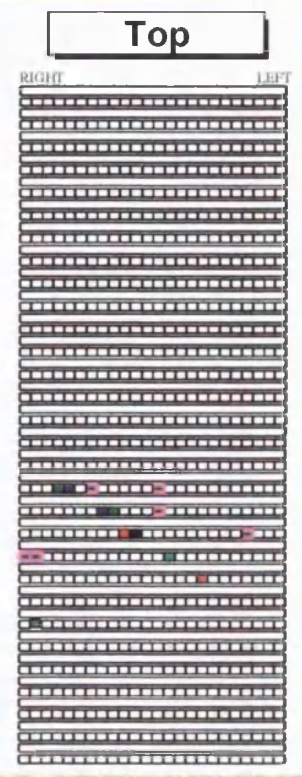

Bottom

RICAT

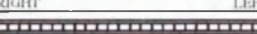
플

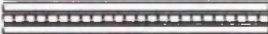

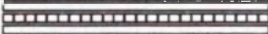
표110101010

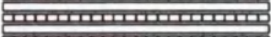

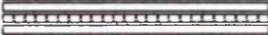
| 年1 2010

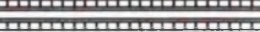

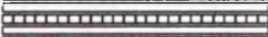

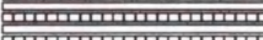

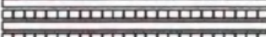
1.1.

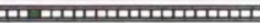

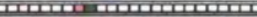

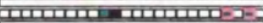
| (111010111010

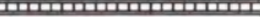

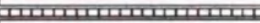

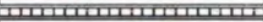

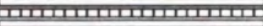

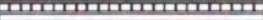

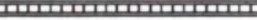

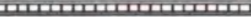

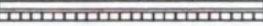

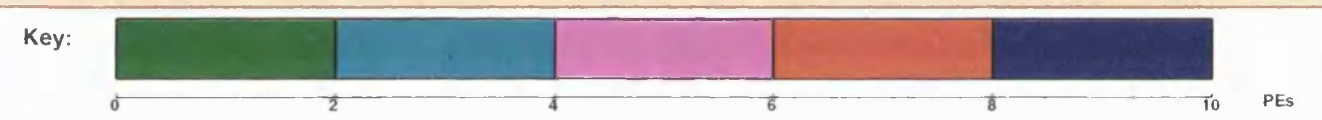

Figure 7.23: An event display from the CalDet showing a cosmic ray muon. 'Left' and 'Right' refer to the ends of the horizontal strips, 'Top' and 'Bottom' to the ends of the vertical strips. The hits highlighted in pink are prospective crosstalk hits. These 'fake' hits in the detector make muon tracking more difficult.

hit the crosstalk hit and source hit can be of similar size due to the discrete nature of photoelectron counting. Using the pulse height, in photoelectrons, to weight the strips, the amount of crosstalk included in the track can be minimized. This is illustrated by Figure 7.24, where the strips tracked by the algorithm are highlighted in yellow. As in Figure 7.23 the prospective crosstalk hits are highlighted in pink. The single un-highlighted strip in the event is the result of unrelated noise, either from a low energy neutron in the detector or dark noise in the phototube.

\section{Strip Orientations and Angular Distributions}

The orientation of scintillator strips at the CalDet results in a problem for strip to strip calibration using cosmic ray muons, not present for the two other MINOS 


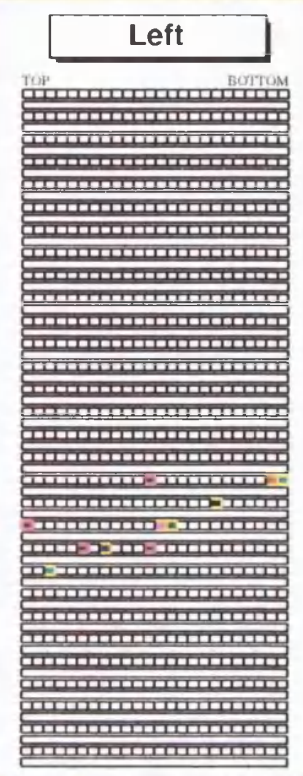

\begin{tabular}{|c|}
\hline Right \\
\hline momen \\
\hline 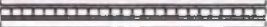 \\
\hline 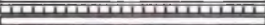 \\
\hline 10 \\
\hline 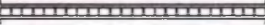 \\
\hline 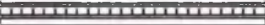 \\
\hline 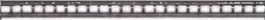 \\
\hline 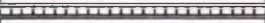 \\
\hline | \\
\hline (1) \\
\hline 210101010101010101010 \\
\hline 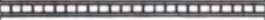 \\
\hline 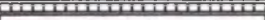 \\
\hline 年 \\
\hline 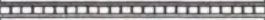 \\
\hline 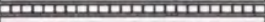 \\
\hline 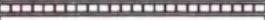 \\
\hline 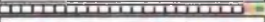 \\
\hline 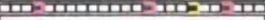 \\
\hline - \\
\hline 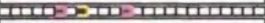 \\
\hline 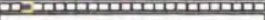 \\
\hline |س \\
\hline (101010 \\
\hline 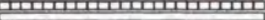 \\
\hline 要1 \\
\hline | \\
\hline | \\
\hline IIIIIIIIIIIIIIIIIIIII) \\
\hline \\
\hline
\end{tabular}
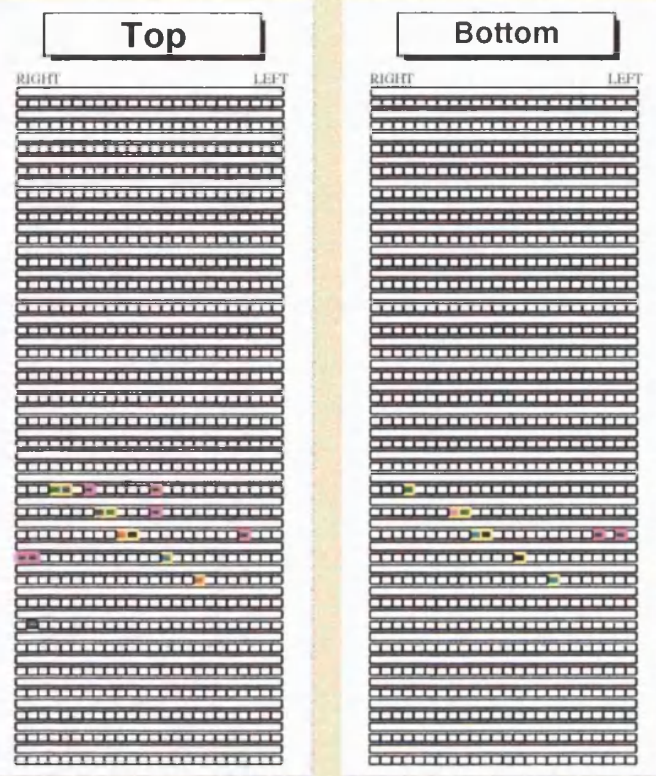

Key:
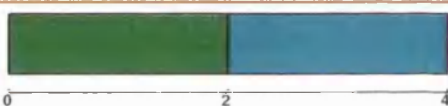

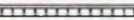
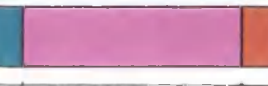

PEs

Figure 7.24: An event display from the CalDet showing a tracked cosmic ray muon. 'Left' and 'Right' refer to the ends of the horizontal strips, 'Top' and 'Bottom' to the ends of the vertical strips. The hits highlighted in yellow are those which are included in the muon track, which include all of the hits that lie along the logical path of the muon. The hits highlighted in pink are prospective crosstalk hits. The un-highlighted strip is the result of random unrelated noise, most likely from dark noise in the phototubes.

detectors. At the CalDet the scintillator strips either run parallel (horizontal) or perpendicular (vertical) to the ground, whereas both orientations of strips in the Near and Far Detectors are at $45^{\circ}$ to the horizontal, see Figure 7.25.

Due to the orientation of the strips the 'natural' angles measured by the CalDet are the vertical and horizontal angles. These angles are formed by a two-dimensional projection of the track in terms of either the horizontal or vertical strips, as shown in Figure 7.26. Note that the horizontal strips are used to measure the vertical angle and vice versa.

The CalDet strip orientations would have no bearing on the strip to strip calibra- 
a) CalDet Strip Orientation

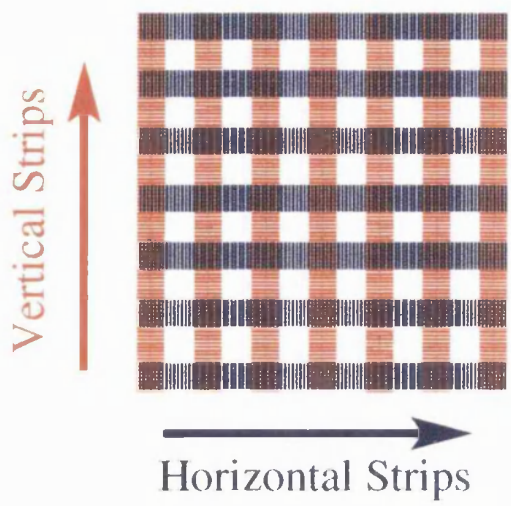

b) Near and Far Detector Strip Orientation

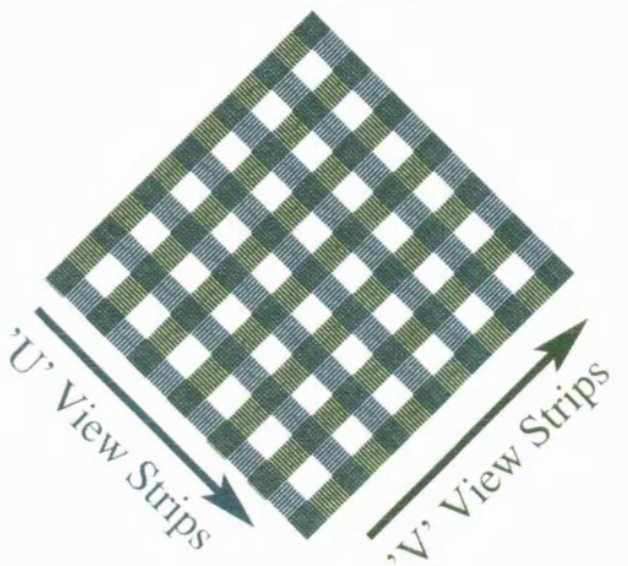

Figure 7.25: At the CalDet (left) the scintillator strips run horizontally and vertically. In the Near and Far Detectors the two orientations, ' $U$ ' and ' $V$ ', are each at $45^{\circ}$ to the horizontal.

tion if it were not for the large angular asymmetry in the cosmic ray muon spectrum. This angular asymmetry stems from the fact that all cosmic ray muons are travelling downwards, therefore at angles close to vertical. The horizontal and vertical angles, with respect to the beamline, are plotted for a sample of cosmic muons in the CalDet in Figure 7.27. The majority of muons have a large vertical angle as they traverse the CalDet. For a muon travelling downwards, negative values of the vertical angle imply the muon was travelling in the same direction as the beam, and positive values imply the opposite direction. Also shown in Figure 7.27 are the natural angles for these muons if the CalDet strips were of the same orientation as those in the Near and Far Detectors.

\section{Performance of Cosmic Muon Calibration Constants}

The energy deposited per plane by $1.8 \mathrm{GeV}$ stopping beam muons, in ADC, is plotted as a function of plane in Figure 7.28, with even and odd planes plotted separately. The muon is expected to deposit less energy per plane, as it loses energy passing through the detector. However, at the end of the track when the muon is travelling slowest it leaves the largest energy deposits, as it spends longer 


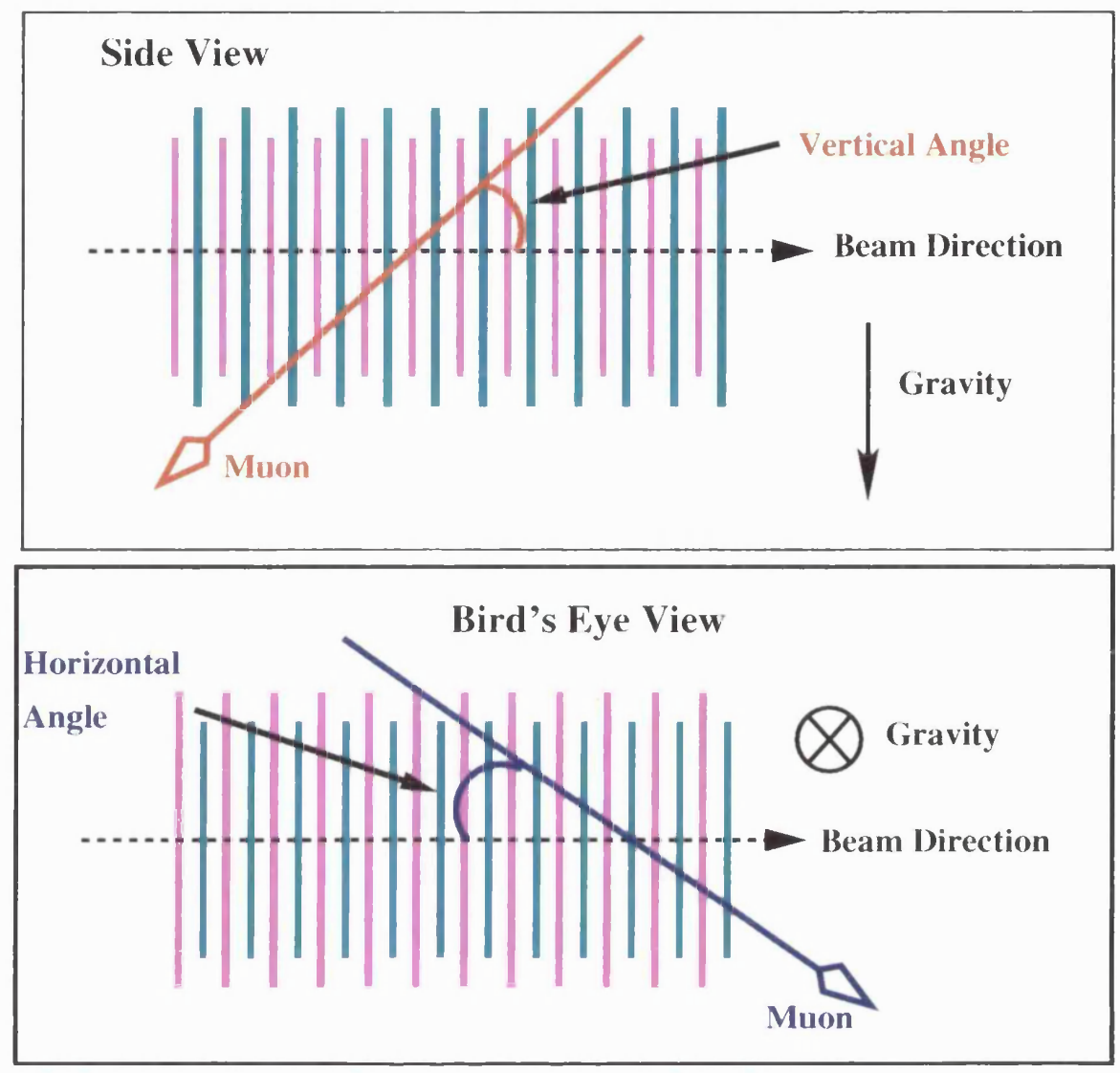

Figure 7.26: Due to the orientation of the strips at the CalDet, the natural angles for the strips are the horizontal and vertical angles shown. These angles are formed by twodimensional projection of the track in terms of planes and strips. The horizontal angle is measured using the vertical strips and vice versa.

travelling through each plane and causes more ionization. For $1.8 \mathrm{GeV}$ muons the minimum ionizing point should be after $\sim 50$ planes. In order to gauge the scatter of the distribution, a straight line is fitted between planes 1 and 39 and residuals are calculated. These residuals are also shown in Figure 7.28, both as a function of plane and as a histogram with a width of $\sim 12 \%$. There is no obvious asymmetry between the even and odd planes, whose residuals are shown in the shaded histograms.

Strip to strip calibration constants were created using the mean of the observed muon ADC spectrum in each strip-end. To test the performance of these calibration constants they were applied to the sample of $1.8 \mathrm{GeV}$ stopping beam muons. 


\section{Angular Distribution of Cosmic Ray Muons in CalDet}

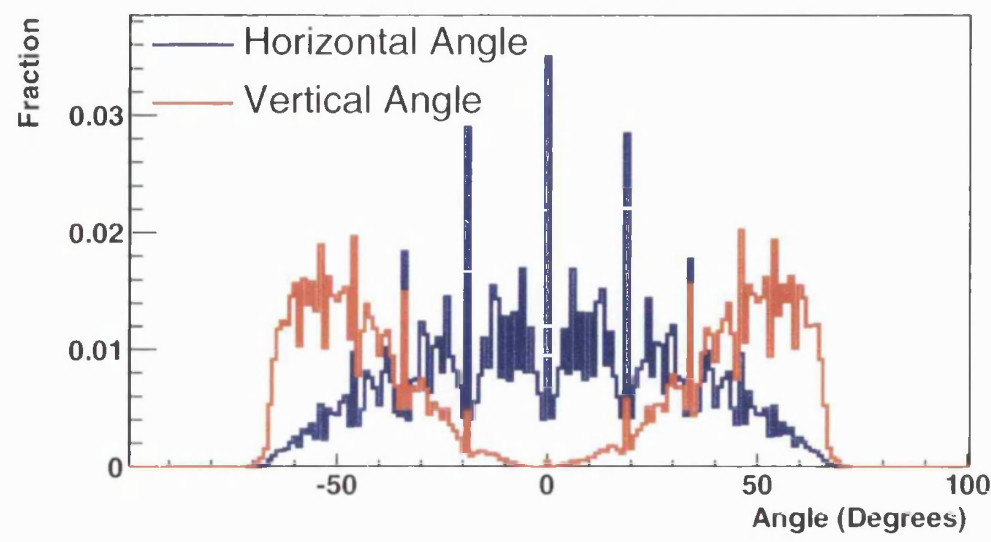

Angular Distribution using Transformed Angles

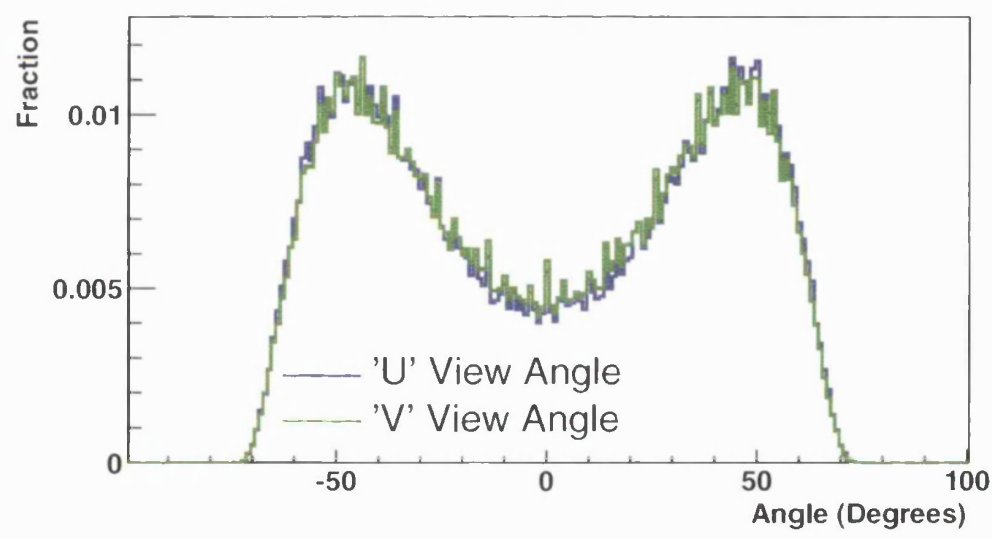

Figure 7.27: The angular distribution of cosmic ray muons at CalDet. The top plot shows the angular distribution in terms of horizontal and vertical angles, which are the natural angles measured using the CalDet strip orientations. As all of the cosmic rays are travelling downwards there is a large asymmetry between the horizontal and vertical angles. For a downwards going muon, negative vertical angles imply muons travelling in the same direction as the beam and positive values the opposite direction. The spikes in the angular distribution are the result of finite resolution in the angle measurement, due to the $4.1 \mathrm{~cm}$ strip width. The bottom plot shows the natural angles for ' $U$ ' and ' $V$ ' strip orientations, if the CalDet planes were in this orientation the two natural angular distributions would be similar. 


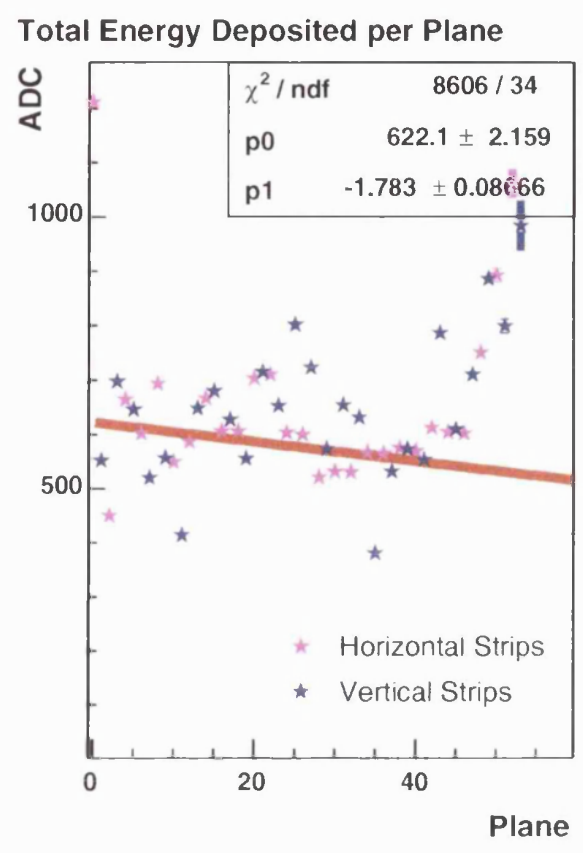

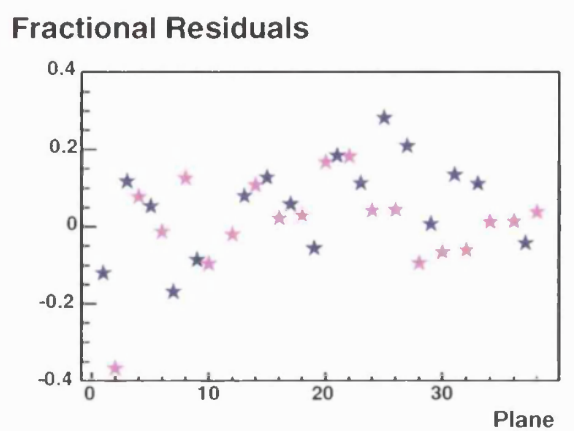

Fractional Residuals

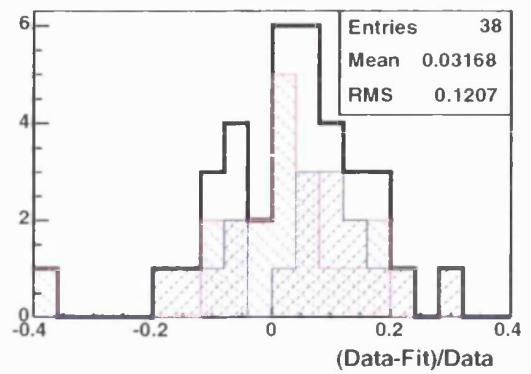

Figure 7.28: The total energy deposited per scintillator plane by a sample of $1.8 \mathrm{GeV}$ stopping beam muons, in ADC. Even and odd planes, which consist of horizontal and vertical strips respectively, are plotted in different colours. The muons are expected to deposit less energy per plane as they lose energy traversing the detector, before slowing significantly and depositing more energy in the last few planes before stopping. Fractional residuals are shown to a straight line fit between planes 1 and 39, both as a function of plane and as a histogram with a width of $\sim 12 \%$. The shaded areas in the residual histogram correspond to the even and odd planes, between which there is no obvious asymmetry.

The total energy deposited per plane by the beam muons, in terms of arbitrary cosmic muon energy units (MEU), is shown in Figure 7.29. The overall scale of the MEU calibration is determined by the (unknown) energy distribution of cosmic ray muons used to create the calibration constants, a relative energy scale calibration must be performed before measurements can be compared between detectors. The calibration removes a large fraction of the scatter visible in Figure 7.28, reducing the width of the residual distribution to $4 \%$. The detectors were designed to achieve 
a relative energy calibration of $2 \%$, in order to achieve this it is desirable to have each plane calibrated to this level as low energy events only deposit energy in a few planes. Therefore the $4 \%$ width of the residual distribution is larger than is desirable. Also the calibration introduces a bias between even and odd planes, which consist of horizontal and vertical strips respectively. This is a direct consequence of the cosmic muon angular asymmetry.
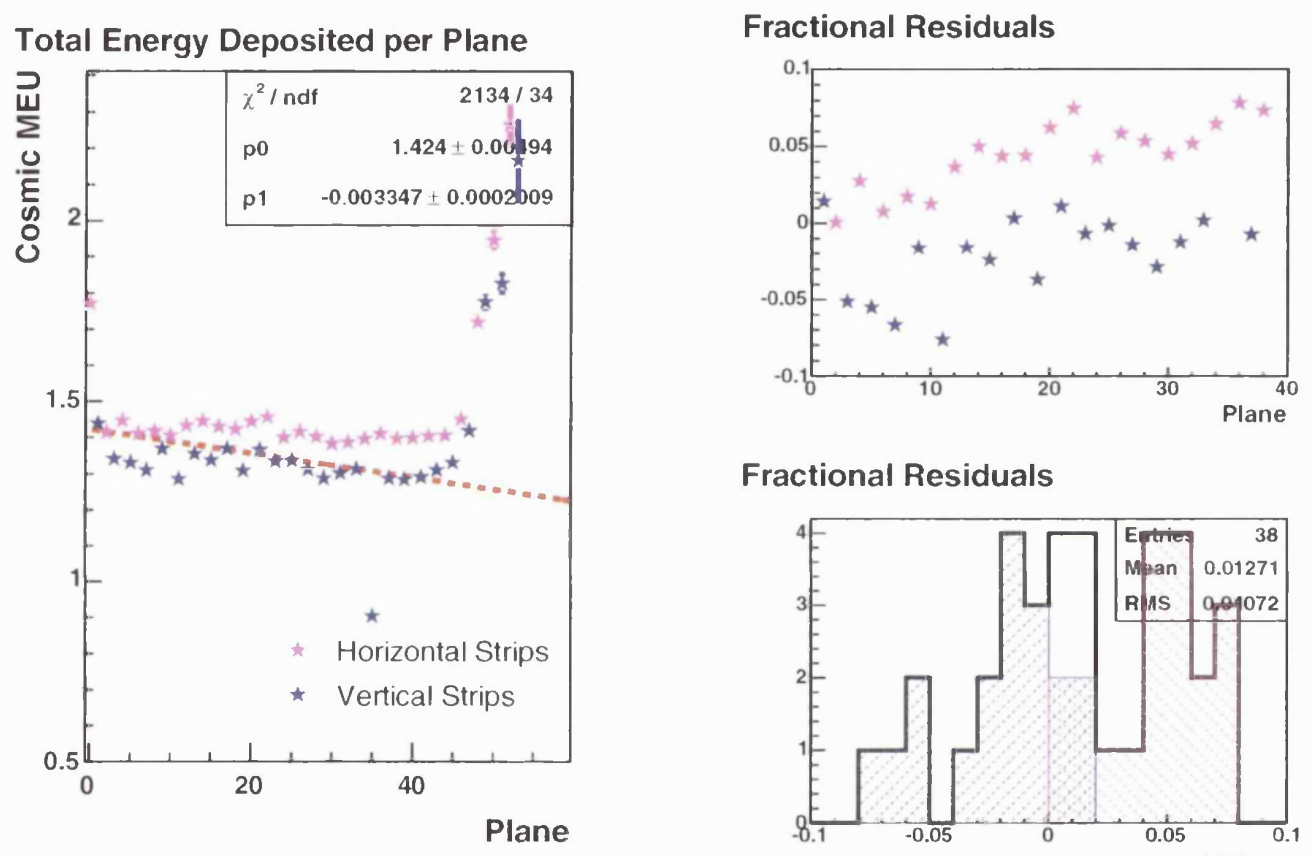

\section{Fractional Residuals}

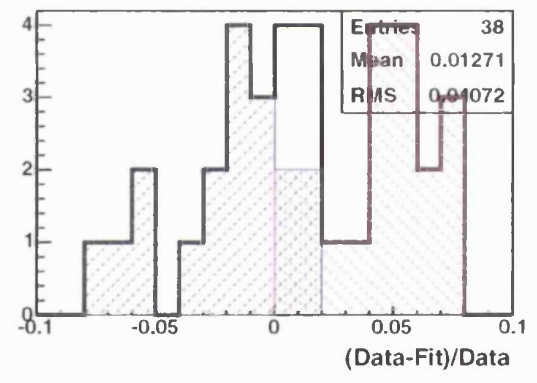

Figure 7.29: The performance of cosmic muon calibration constants. The total energy deposited per scintillator plane by a sample of $1.8 \mathrm{GeV}$ stopping beam muons, in cosmic MEU. The width of the fractional residual, to a straight line fit between planes 1 and 39 , is reduced to $4 \%$ from the $12 \%$ shown in Figure 7.28. However, the cosmic calibration introduces a bias between the even and odd planes, which consist of horizontal and vertical strips respectively, whose residuals are plotted separately in the shaded histograms. This bias is a direct consequence of the angular asymmetry visible in Figure 7.27. 


\section{Path Length Through Scintillator}

The number of photons produced by a muon passing through a piece of scintillator is related to the muon's path length through the scintillator and to a first approximation this relationship is linear. For a strip to strip calibration to be performed, the path length distribution must be the same in each strip or a correction must be applied to account for any differences.

If the CalDet scintillator strips were infinitely wide and long the average path length through a scintillator strip, $\langle d s\rangle$, would be given by

$$
\langle d s\rangle=\frac{d s}{d z} \Delta z
$$

where $d s^{2}=d x^{2}+d y^{2}+d z^{2}$ and $\Delta z=1 \mathrm{~cm}$ is the thickness of the scintillator plane.

However, due to the finite dimensions of the scintillator strips the average path length through a strip is smaller than the above value. Muons can traverse two or more scintillator strips in a plane, or 'corner clip', as illustrated in Figure 7.30. Using purely geometrical considerations the average path length for a fixed angle, $\theta$, can be calculated [70]. Assuming that the muon entry point across the transverse width of the strip, $y$, is a uniform distribution, the average path length through a scintillator strip can be obtained by integrating over all possible entry positions:

$$
\begin{aligned}
\langle d s\rangle & =\frac{\int_{0}^{\Delta y+\delta y} d s(y) d y}{\int_{0}^{\Delta y+\delta y} d y} \\
& =\frac{d s}{d z} \Delta z\left(\frac{\Delta y}{\Delta y+\Delta z\left|\frac{d y}{d z}\right|}\right),
\end{aligned}
$$

where $\delta y$ is the maximum y position for which the muon will still pass through the strip, $\Delta y=4.1 \mathrm{~cm}$ is the transverse strip width and $\frac{d y}{d z}=\tan \theta$.

Due to the large asymmetry between the horizontal and vertical angular distributions, the average path length in horizontal and vertical strips is not the same. The vertical angular distribution is peaked at higher values than the horizontal distribution which leads to a shorter average path length in the horizontal strips due to the increased chance of corner clipping muons.

The different path length distributions of cosmic ray muons in the horizontal and vertical strips mean that a path length correction must be applied before these 


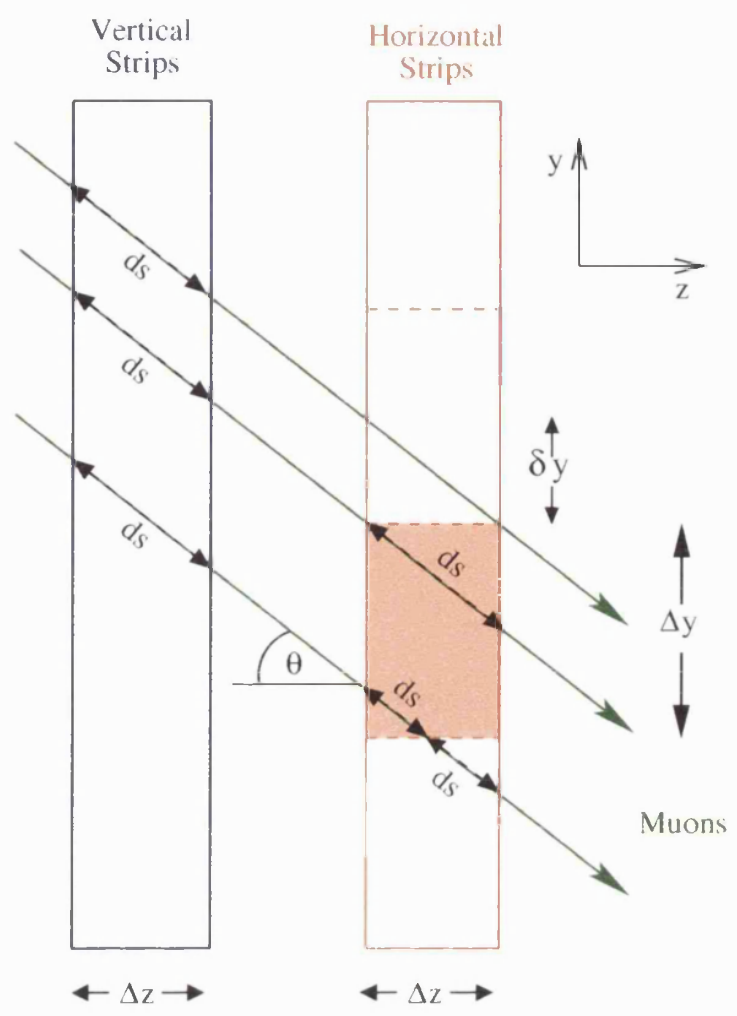

Figure 7.30: A sketch showing how the average path length varies between the horizontal and vertical strips. The average path length in the horizontal strips is smaller as there is a larger chance that the muon will traverse two or more scintillator strips. In the MINOS detectors the plane thickness, $\Delta z$, is $1 \mathrm{~cm}$ and the transverse strip width, $\Delta y$, is $4.1 \mathrm{~cm}$

muons can be used to perform a strip to strip calibration. The correction, taken from Equation 7.21, could either be applied on an event by event basis or on a hit by hit basis. It is preferable to apply the correction on a hit by hit basis as muons are susceptible to sudden changes in direction due to multiple scattering in the steel planes. A local angle at each plane, determined from the track position in surrounding planes, is used to calculate the path length correction on a hit by hit basis. The normalized corrected and uncorrected mean ADC as a function of path length through the scintillator plane are shown in Figure 7.31. Before applying the correction, variations of $\sim 90 \%$ are seen across the observed range of path lengths; after correction this dependence is reduced to $\sim 6 \%$.

For either the Near or Far Detectors this path length correction would be suf- 


\section{Pulse Height Dependence on Path Length through Scintillator Plane}

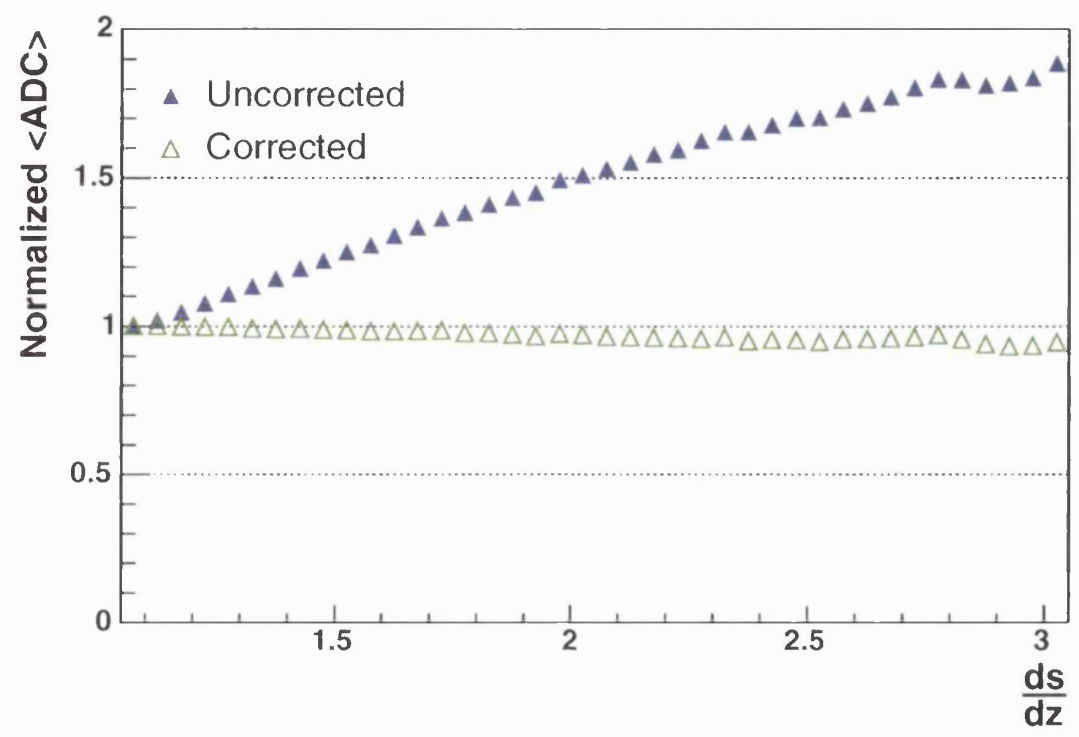

Figure 7.31: The normalized mean ADC from cosmic ray muon spectra, as a function of path length through the scintillator plane. Both the uncorrected mean ADC and the mean after path length correction are shown. Before correction the mean ADC increases by $\sim 90 \%$ over the observable range of path lengths, after correction there is an $\sim 6 \%$ decrease

ficient as the angular distribution of cosmic ray muons is similar across all strips. However, due to the large asymmetry between the horizontal and vertical angles at CalDet this correction is not sufficient. This is illustrated in Figure 7.32, where the mean $\mathrm{ADC}$ before and after correction is plotted separately for the horizontal and vertical strips. The corrected response of the horizontal and vertical strips differs by up to $\sim 5 \%$.

There are a number of factors which contribute to the observed difference in muon response, after path length correction, between the horizontal and vertical strips. These factors include:

Zero reconstruction The difference in path length distributions, due to the increased likelihood of comer clipping muons in the horizontal strips, leads to a different probability of recording zero photoelectrons in the PMTs. 


\section{Horizontal - Vertical Strip Asymmetry in Muon Response}

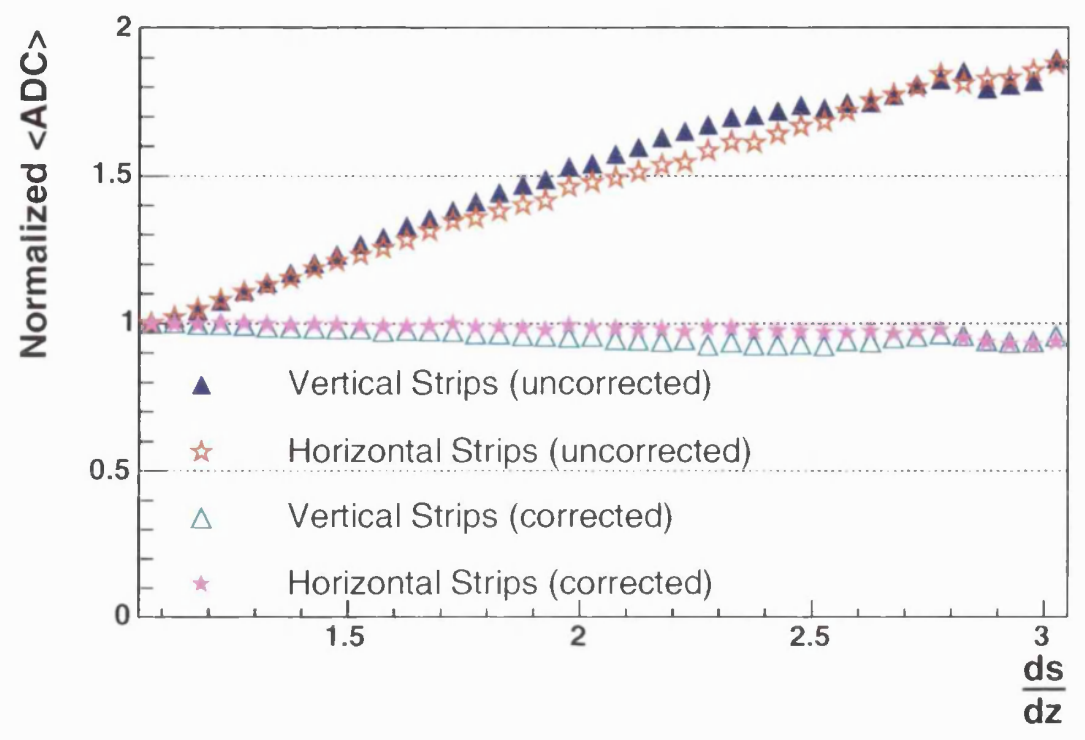

Horizontal - Vertical Strip Asymmetry in Muon Response (Zoomed)

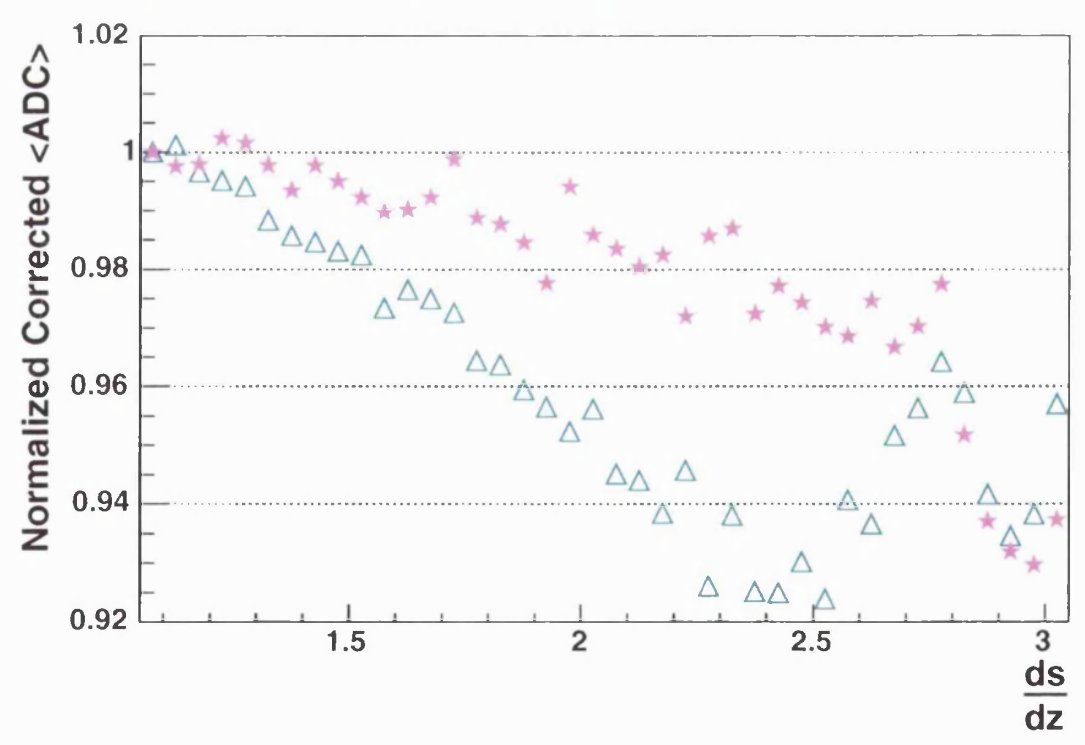

Figure 7.32: The normalized mean ADC from cosmic ray muon spectra, as a function of muon path length through the scintillator plane is shown separately for horizontal and vertical strips in the top plot. The bottom plot is a close up of the corrected response, showing a $\sim 5 \%$ deviation between the horizontal and vertical strips. 
Attenuation The response of the scintillator strip varies across its transverse length due to attenuation of the signal in the wavelength shifting fibre and also due to geometrical concerns. The distribution of transverse position across the strip length is different between the horizontal and vertical strips as most of the muons enter the top of the detector.

Proximity to wavelength shifting fibre The response also varies across transverse width of the scintillator strip due to the acceptance of the wavelength shifting fibre. This varies between the strip orientations due to the differing angular distributions.

Energy loss As the muon traverses the detector, from top to bottom, it loses energy, for low energy muons this results in different energy deposits between the highest and lowest strips in the detector. No evidence of this energy loss has been seen with cosmic ray muons exiting the detector.

Crosstalk Despite the robustness of the muon tracker with regards to not including crosstalk, there are a certain class of crosstalk hits that are included. These are the crosstalk hits that, due to the pixel to strip wiring, happen to lie along the path of the muon. These hits are very difficult to remove, particularly as they might contain charge both from crosstalk and also from energy deposits of the muon.

\section{Zero reconstruction}

To reduce data rates to a manageable level the MINOS electronics employ an online zero suppression, or sparsification. Signals below a certain threshold value are discarded in the electronics and not stored in the data stream. This means that the number of zero photoelectrons measured by the PMTs must be reconstructed offline. Zero reconstruction is important when creating strip to strip calibration constants; if one does not reconstruct zeros, or incorrectly reconstructs them, a bias between high and low light output strips can be introduced. This bias arises 
because the low light output strips see more occurrences of zero photoelectrons than the higher light output strips.

If the zeros are not included in the spectra the measured means are higher than the true mean, with lower light output strips affected more than higher light output strips. This is illustrated in Figure 7.33, assuming a purely Poissonian distribution of photoelectrons from the muon spectra. The fractional deviation of the measured mean without zeros from the true mean of the Poisson distribution is plotted as a function of light output, over the range of light outputs seen at CalDet. The fractional deviation has an exponential dependence on light level ranging from $15 \%$ for the lowest light output strips to less than $1 \%$ for the highest. This approximation is clearly an over simplification of the muon spectra, but does give an idea of the magnitude of the effect.

\section{Effect of Zero Reconstruction on Mean of Muon Spectra}

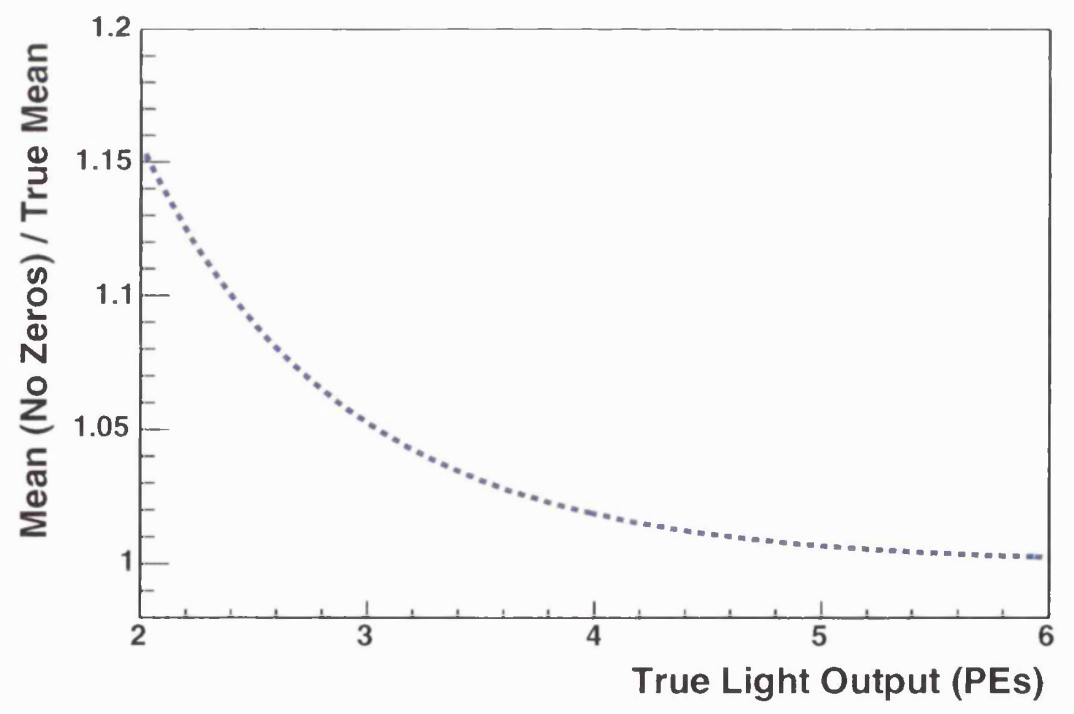

Figure 7.33: The fractional deviation from truth of the mean of the muon ADC spectra when zeros are not included in the determination of the mean of the distributions. The muon spectra were approximated by a purely Poissonian distribution, to gauge the magnitude of the effect. The absence, or imperfect implementation, of zero reconstruction results in a bias between high and low light output strips.

During the running with Far Detector PMTs and electronics at the CalDet, both 
ends of the scintillator strips were read out. This double ended readout provides a handle for zero reconstruction. When one end of the strip has a signal above threshold and the other end does not, a zero is added to the spectrum of the end which did not read out. Using this method of zero reconstruction the only zeros not reconstructed are those that occur when both ends of the scintillator strip are below threshold. If the muon spectra were simple Poisson distributions then even for the lowest light output strips, zero photoelectrons on both ends of the strip would only occur less than $1 \%$ of the time. However, the muon spectra are not simple Poisson distributions and the percentage of double ended zeros is greater than $1 \%$ even for some of the highest light output strips. Muon spectra are convolutions of many Poisson distributions. Even for a single angle of incidence to the strip,the muon spectrum is formed from many Poisson distributions corresponding to the different path lengths through the scintillator arising from corner clipping muons. The observed muon spectrum is made up of the combination of spectra from all of the different angles. Due to the presence of very short path lengths in the scintillator strips, from corner clipping muons, the fraction of hits with zero photoelectrons on both ends is higher than one would expect from Poisson statistics using the mean light output of the strip.

Imperfect zero reconstruction can also introduce a bias between horizontal and vertical strips. The average path length is lower in the horizontal strips and hence the fraction of short path lengths, which contribute most of the double ended zeros, is higher. The fraction of zeros has been estimated using a simple simulation of the light output of the scintillator strips, and subsequent photo-multiplication. The simulation used the CalDet strip geometry and the measured muon angular distribution. Figure 7.34 shows the simulated fraction of zeros as a function of light output for the two strip orientations, compared with the fraction of zeros measured using single sided hits in the CalDet data. The simulation predicts a greater fraction of zeros for the horizontal strips than for the vertical strips. This is not seen in the data, as single sided hits are a poor estimator of the number of zeros seen in the cosmic spectra. For very short path lengths there is a high chance that neither 
end will read out above threshold and it is impossible to include these double ended zeros in the muon spectra. However the double ended zeros need to be included in the spectra for an accurate determination of the light output of the strip.

\section{Comparison of Zero Fraction from Simulation and Data (Cosmics)}

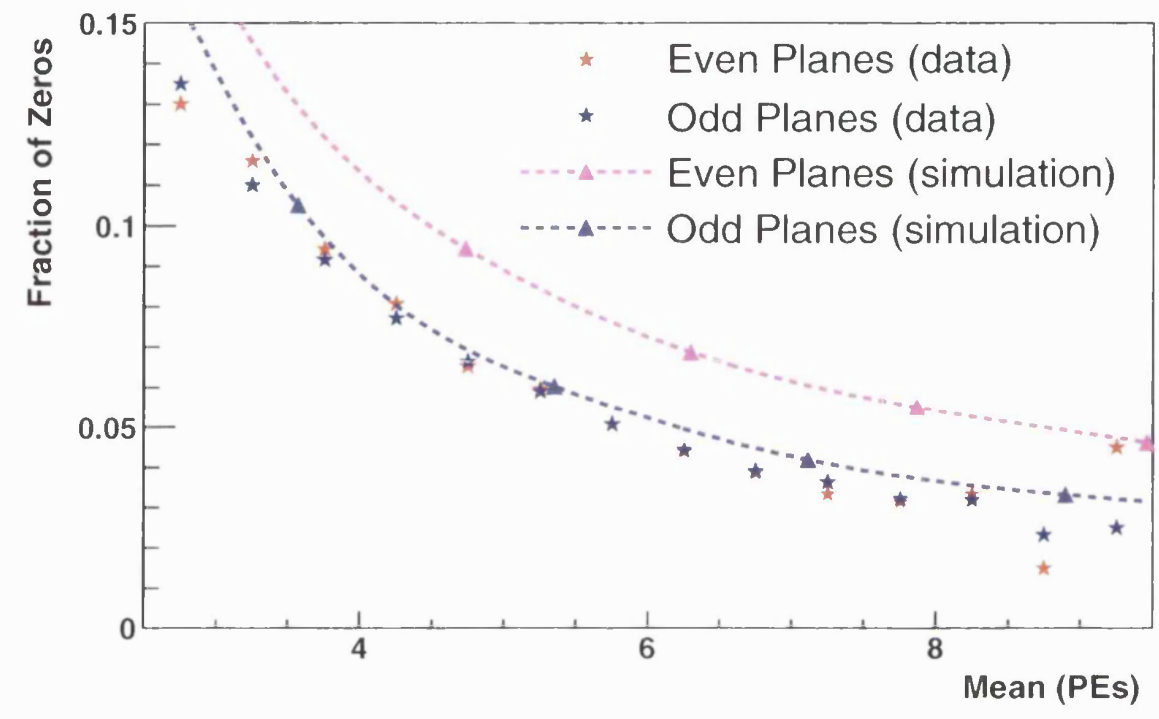

Figure 7.34: The fraction of zero photoelectrons predicted from a simulation of the scintillator strip light output, using the CalDet strip geometry and angular distribution of cosmic muons, is compared to the fraction estimated in the data using single ended hits. The simulation predicts a greater fraction of zeros for the horizontal strips, due to the shorter path lengths from corner clipping muons. This is not seen in the data using single ended hits to determine the fraction of zeros, as for very short path lengths there is a high chance that neither end will read out above threshold.

An alternative approach to zero reconstruction [69] uses the expected number of zero photoelectrons, based on the light output and pathlength distribution in each strip. Using the same arguments as described in the derivation of Equation 7.21, the average probability of obtaining zero photoelectrons, $\left\langle P_{0}\right\rangle$, for a fixed angle is 
given by

$$
\begin{aligned}
\left\langle P_{0}\right\rangle & =\frac{\int_{0}^{\Delta y+\delta y} e^{-\lambda d s(y)} d y}{\int_{0}^{\Delta y+\delta y} d y} \\
& =\frac{e^{-\lambda \frac{d s}{d z} \Delta z}\left(\Delta y-\left|\frac{d y}{d z}\right| \Delta z\right)+\frac{2\left|\frac{d y}{d z}\right|}{\lambda \frac{d s}{d z}}\left(1-e^{-\lambda \frac{d s}{d z} \Delta z}\right)}{\Delta y+\left|\frac{d y}{d z}\right| \Delta z}
\end{aligned}
$$

where $\lambda$ is the mean number of photoelectrons obtained from a muon passing through $1 \mathrm{~cm}$ of scintillator.

For each hit that is used to form the muon ADC spectrum, the ADC value is weighted by the probability of not obtaining zero photoelectrons, $1-\left\langle P_{0}\right\rangle$, and the zero bin is weighted by the probability of having zero photoelectrons, $\left\langle P_{0}\right\rangle$. As this method relies on the number of photoelectrons at the photocathode, the gain of the PMTs and electronics must have already been determined.

In Figure 7.35 the number of zeros in the data, estimated using this weighting method, is compared to the number of zeros measured using the simulation. The agreement between the simulation and the data is much better than if the zero reconstruction is performed using single ended hits.

\section{Attenuation in Scintillator Module}

Some of the photons produced by muons passing through the scintillator module are absorbed by the wavelength shifting fibre embedded in the scintillator strips and are transported out of the module. The amount of light measured by the PMTs is dependent on where along the transverse length of the strip the muon passed, as illustrated in Figure 7.36 for a sample of straight PS muons. The average light output as a function of transverse position across the strip is plotted for the four strip orientation/readout fibre combinations. The distributions are not purely exponential due to large end effects at the edges of the scintillator module. These end effects are due to a lack of reflective coating on the ends of the scintillator module; a significant fraction of the light exits from the ends of the strips and is not absorbed by the wavelength shifting fibre. Although the overall attenuation of those strip-ends read out with green fibre is larger than the clear, there is a shallower 


\section{Comparison of Zero Fraction from Simulation and Data (Cosmics)}

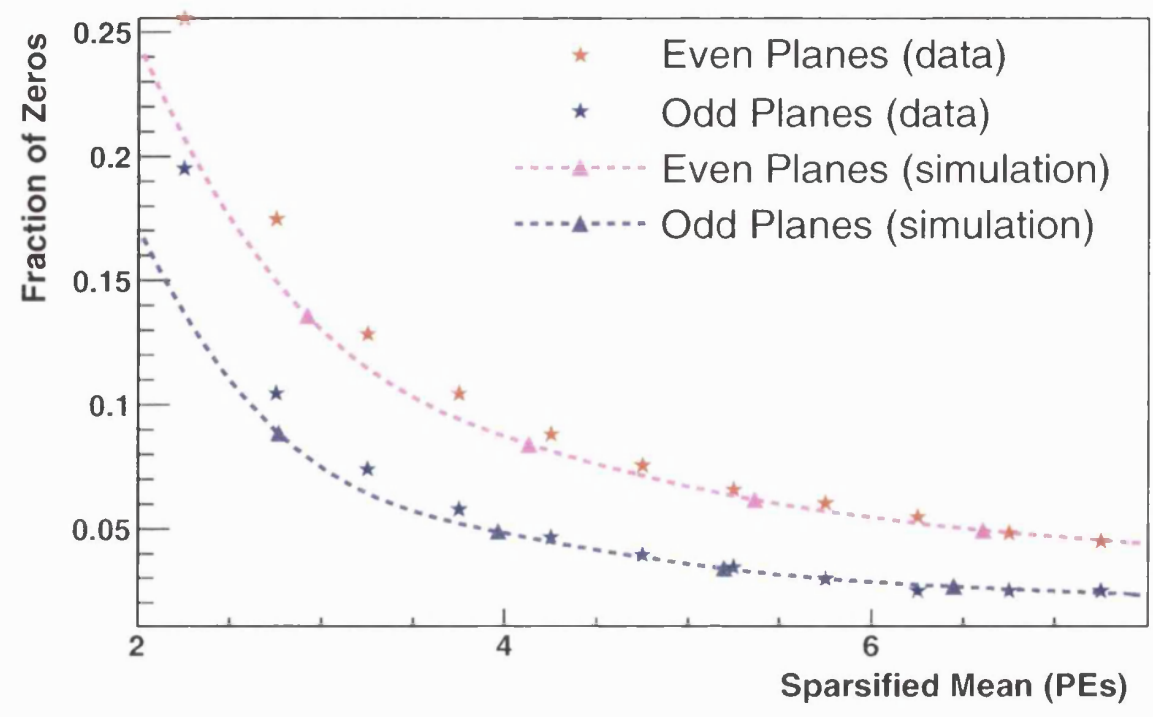

Figure 7.35: Comparison of predicted fraction of zero photoelectrons to the fraction estimated using an alternative zero reconstruction method, as a function of mean light level. The method relies on weighting the zero photoelectron contribution on a hit by hit basis, based upon the strip light output and the path length through the scintillator strip. The data and the simulation agree well at the higher light levels, at lower light levels there are more zeros estimated from the data than predicted by the simulation.

attenuation curve in the centre of the module. This is due to the long and short components of the wavelength shifting fibre's attenuation. The distributions are parameterized using a ninth order polynomial and these parameterizations can be used to remove the attenuation effect from the cosmic ray muons, or other particles.

\section{Performance of Corrected Cosmic Calibration Constants}

The performance of cosmic muon calibration constants, after path length, zero reconstruction and attenuation corrections, has been tested using a sample of $1.8 \mathrm{GeV}$ beam muons. The energy deposited per plane of scintillator, in cosmic MEU, is shown in Figure 7.37. These calibration constants are a clear improvement over those shown in Figure 7.29, the width of the fractional residual distribution is re- 

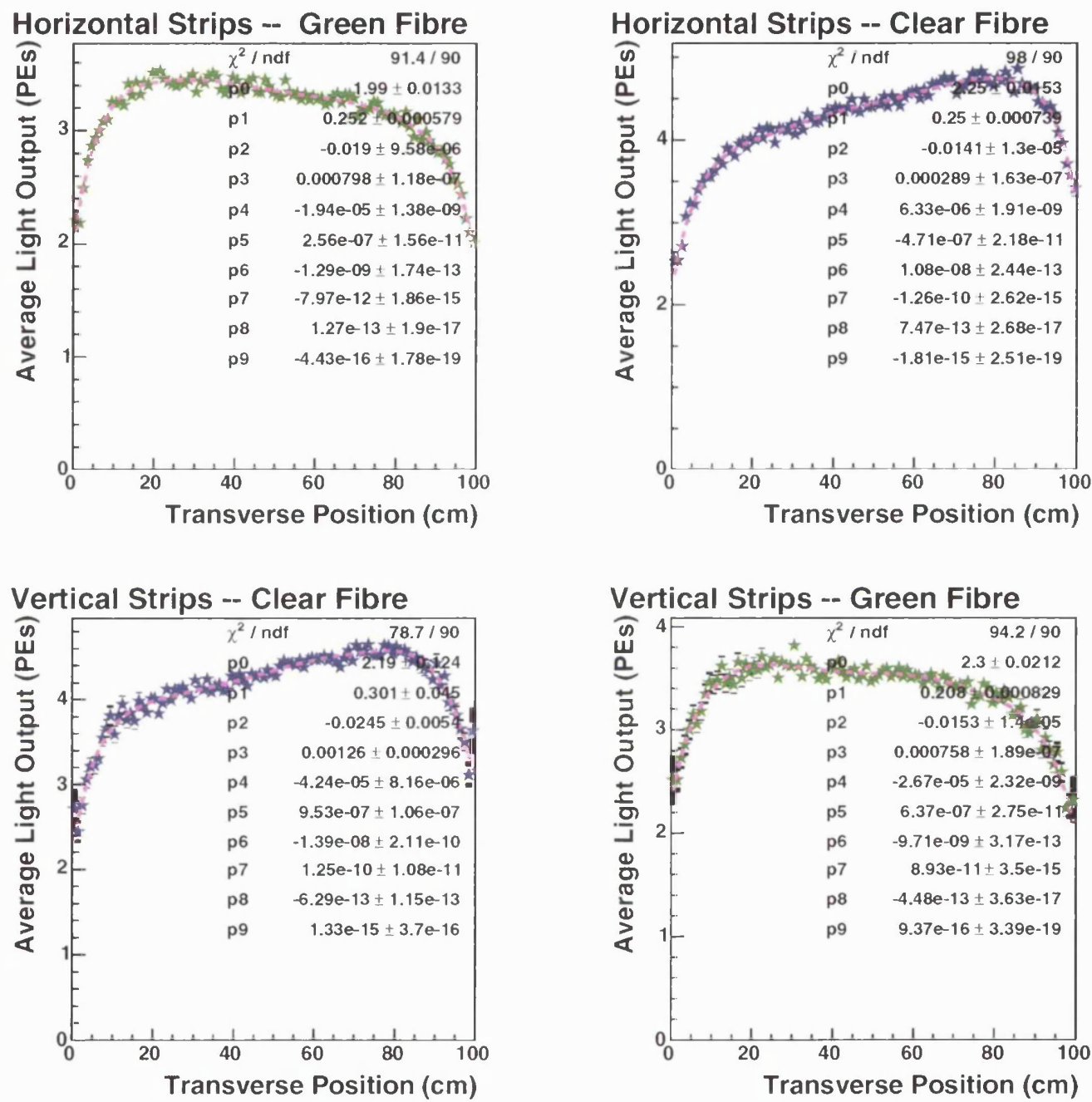

Figure 7.36: The attenuation of muon light output as a function of transverse position across strip length. The four strip orientation and readout cable combinations are plotted separately. The turn down in light output at the edges of the strips is due to a lack of reflective coating on the ends of the strips, where light escapes the scintillator strips and is not absorbed by the wavelength shifting fibre. The distributions are parameterized using a ninth order polynomial, these parameterizations can be used to remove the attenuation effect. 
duced to $2 \%$ with no obvious even odd asymmetry. This is the desired level for a relative calibration of the detector. However, certain anomalous channels, such as planes 0 and 35 , have calibration constants which do not fully remove the nonuniformity of the detector.
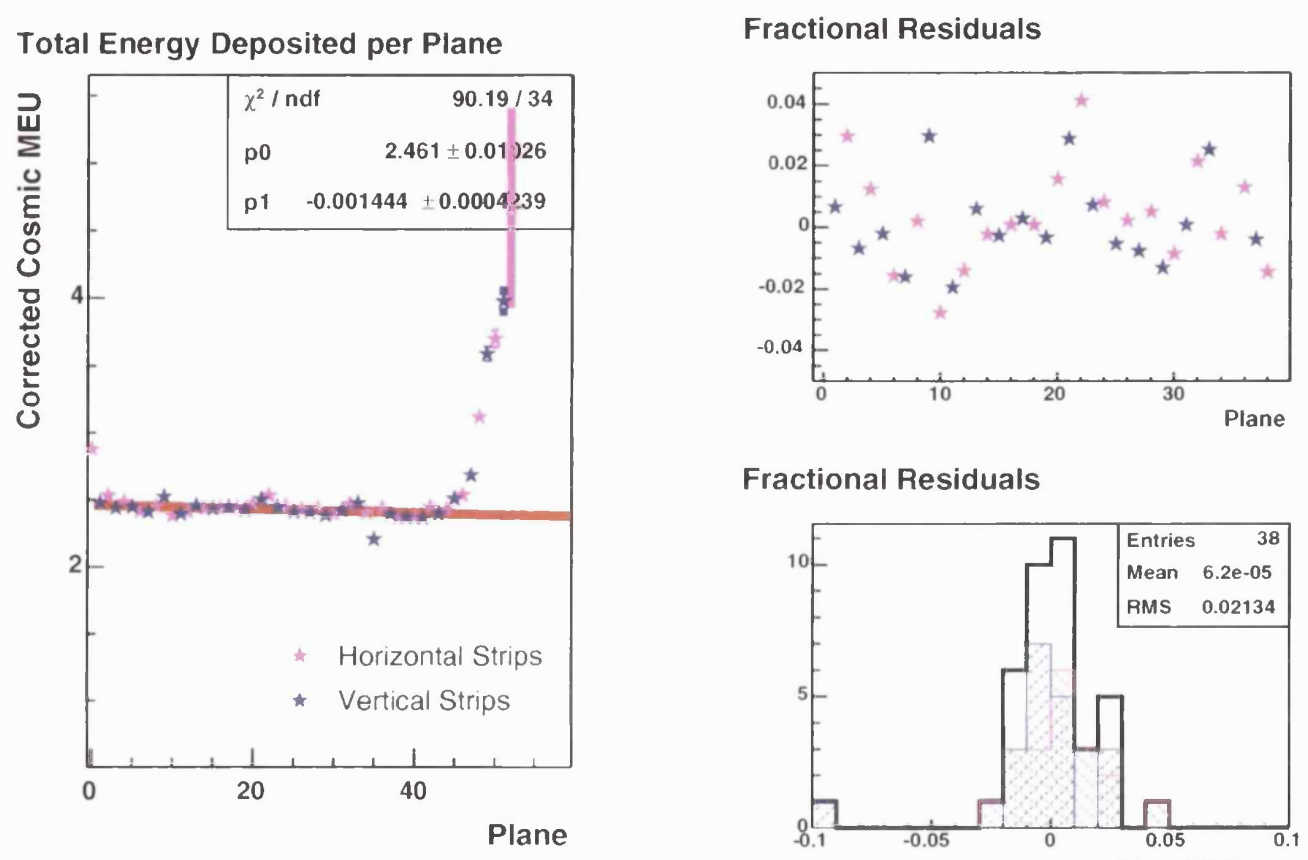

\section{Fractional Residuals}

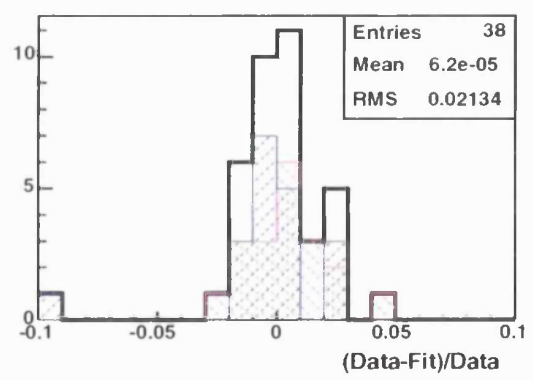

Figure 7.37: The performance of cosmic muon calibration constants, created using muon ADC spectra corrected for path length and attenuation in scintillator strip and including reconstructed zeros. The total energy deposited per scintillator plane by a sample of $1.8 \mathrm{GeV}$ stopping beam muons, in cosmic MEU, is shown. Unlike the uncorrected cosmic constants an even odd bias is not introduced using these constants and the width of the residual distribution is reduced to $2 \%$. However plane 35 , which had an anomalously low light output due to a damaged optical cable, is not well calibrated.

\section{Total Energy Deposited in a Scintillator Plane}

The techniques for obtaining a strip to strip calibration discussed thus far relied on characterizing the response of strip-ends in the detector using the muon spectrum observed in that strip-end. It has been shown that this method is sensitive to the 
path length through the scintillator strip, and requires an accurate zero reconstruction. A method for characterizing the strip-end response using the total energy deposition in one end of a plane, as suggested in [71], has been investigated.

The method relies on an iterative approach to the calibration constant determination. In each plane the muon traverses, the strip of maximal energy deposition is determined using the current calibration constants. All of the energy seen in the plane is then attributed to the strip, considering each end separately and multiplying energy deposits in different strips by the ratio of the calibration constants. Using these newly created strip-end histograms, filled with the total energy deposited in one end of the plane, new calibration constants are formed. These constants are used in the next iteration and the process usually converges after a few iterations.

As this method uses the total energy deposited in the scintillator plane, it is sensitive to the total path length through the scintillator plane, and not the path length through individual scintillator strips. The advantage of this is that it is the same for planes with either horizontal or vertical scintillator strips. However, the total energy observed in a plane of scintillator is not necessarily the same for muons which pass through a single strip and those which corner clip. There are two dominant classes of effect which cause this difference: threshold effects and edge effects at the boundary between scintillator strips. The PMT is only read out if the charge in the PMT is greater than a certain threshold; for corner clipping muons, which can have very short path lengths in the scintillator strips, there is a greater chance that some of the energy will be lost on PMTs below this threshold. Corner clipping muons are also more sensitive to edge effects at the sides of the scintillator strips and these effects tend to decrease the total energy seen. These threshold and edge effects can introduce a bias between the horizontal and vertical strips, due to the larger number of corner clipping muons in the horizontal strips.

\section{Performance of Total Energy in Plane Cosmic Calibration Constants}

The calibration constants, created using the total energy deposited per plane by cosmic muons, have been tested using a sample of $1.8 \mathrm{GeV}$ stopping beam muons. 
The total energy deposited per plane of scintillator, in cosmic MEU, is shown in Figure 7.38. Like the uncorrected cosmic calibration constants, in Figure 7.29, an even-odd bias is introduced using these constants. However the anomalous plane 35 is calibrated much better than with the corrected cosmics as the iterative method used is more robust.
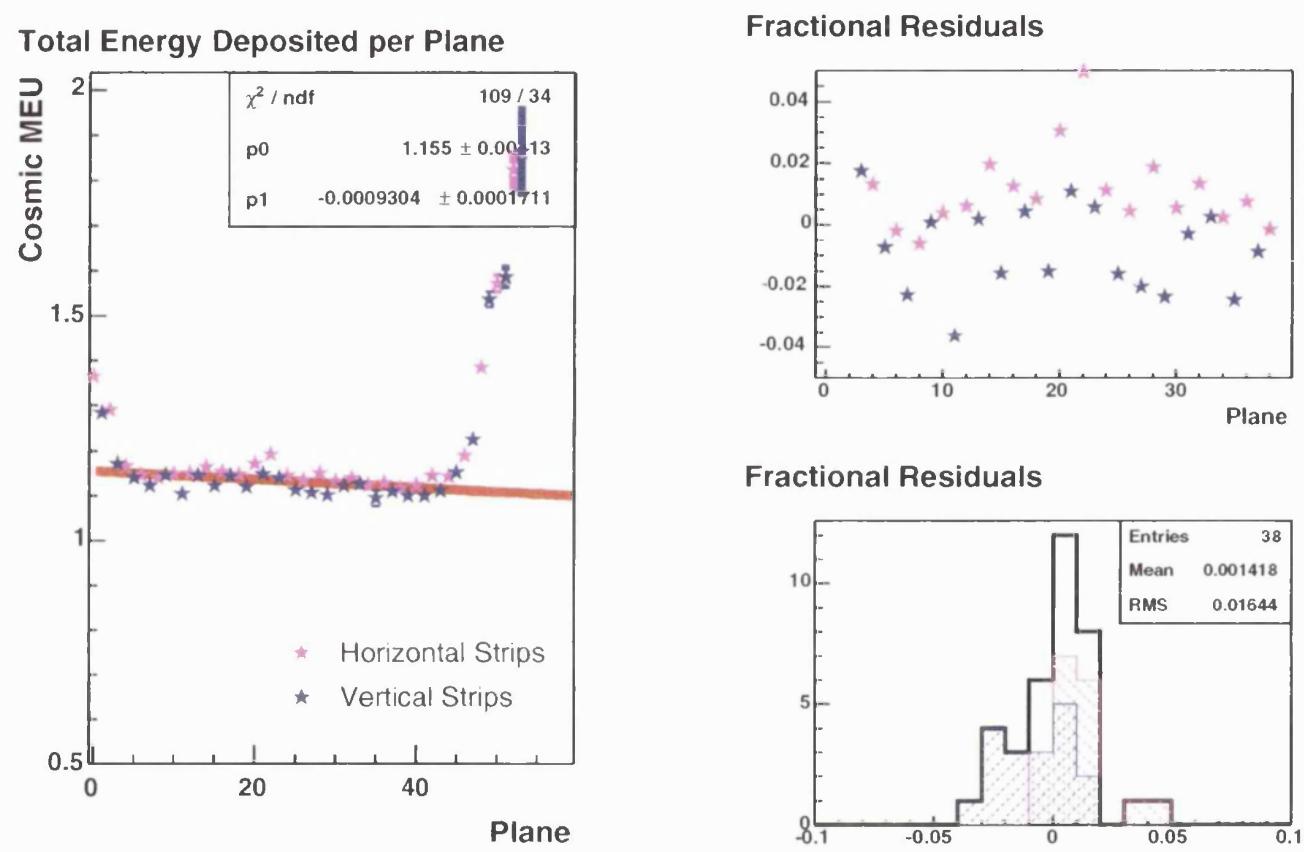

\section{Fractional Residuals}

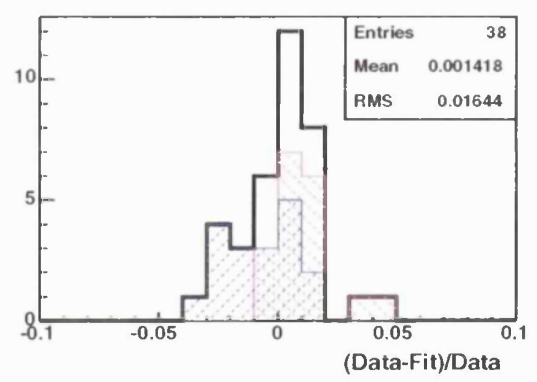

Figure 7.38: The performance of cosmic muon calibration constants, created using the summed total energy deposited per plane from cosmic muons. The total energy deposited per scintillator plane by a sample of $1.8 \mathrm{GeV}$ stopping beam muons, in cosmic MEU, is shown. Although the total width of the residual distribution is less than $2 \%$, a bias is introduced between the even and odd planes this is clearly visible in the residual plots. This bias is smaller than the one shown in Figure 7.29, using constants derived without any corrections. 


\subsubsection{PS Muons in the T7 Testbeam}

The large asymmetry in angular distribution of cosmic muons between the strip orientations at CalDet mean that performing a strip to strip calibration using cosmic ray muons is very difficult. Fortunately there is another source of muons that can be used to calibrate the detector, which does not suffer from such an asymmetry: the PS muons. The PS muons are high energy muons that are created upstream of the testbeam hall and when the CalDet is situated in the $\mathrm{T} 7$ testbeam area these muons enter the detector almost parallel to the beam. Figure 7.39 shows a typical PS muon traversing all 60 planes of the detector. As the muons are travelling at very similar horizontal and vertical angles, they do not suffer from the same biases as the cosmic ray muons.
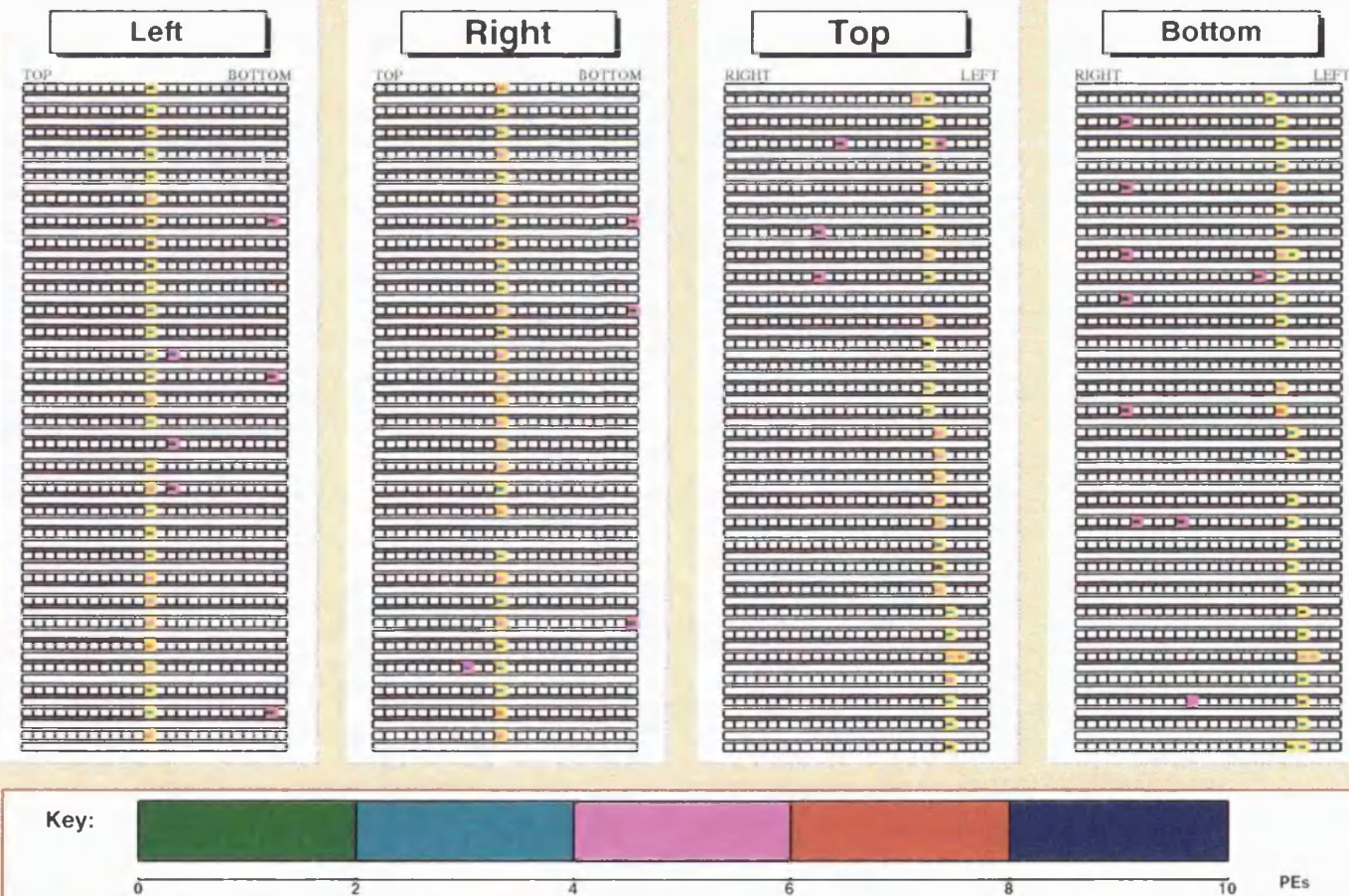

Figure 7.39: An event display showing a PS muon, when the CalDet was in the T7 area, traversing the entire length of the detector. 'Left' and 'Right' refer to the ends of the horizontal strips, 'Top' and 'Bottom' to the ends of the vertical strips. The hits highlighted in yellow are those which are included in the muon track. 


\section{Angles and Path Lengths}

The angular distributions of muons in the $T 7$, when the local beam stop is in but the PS is running, is shown in Figure 7.40. Comparing these distributions to the ones shown in Figure 7.27 showing the angular distribution of cosmics when the PS was switched off, the PS muons are easily identifiable as those in the peaks at low angles.

\section{Muon Angular Distribution from the $\Pi 7$}

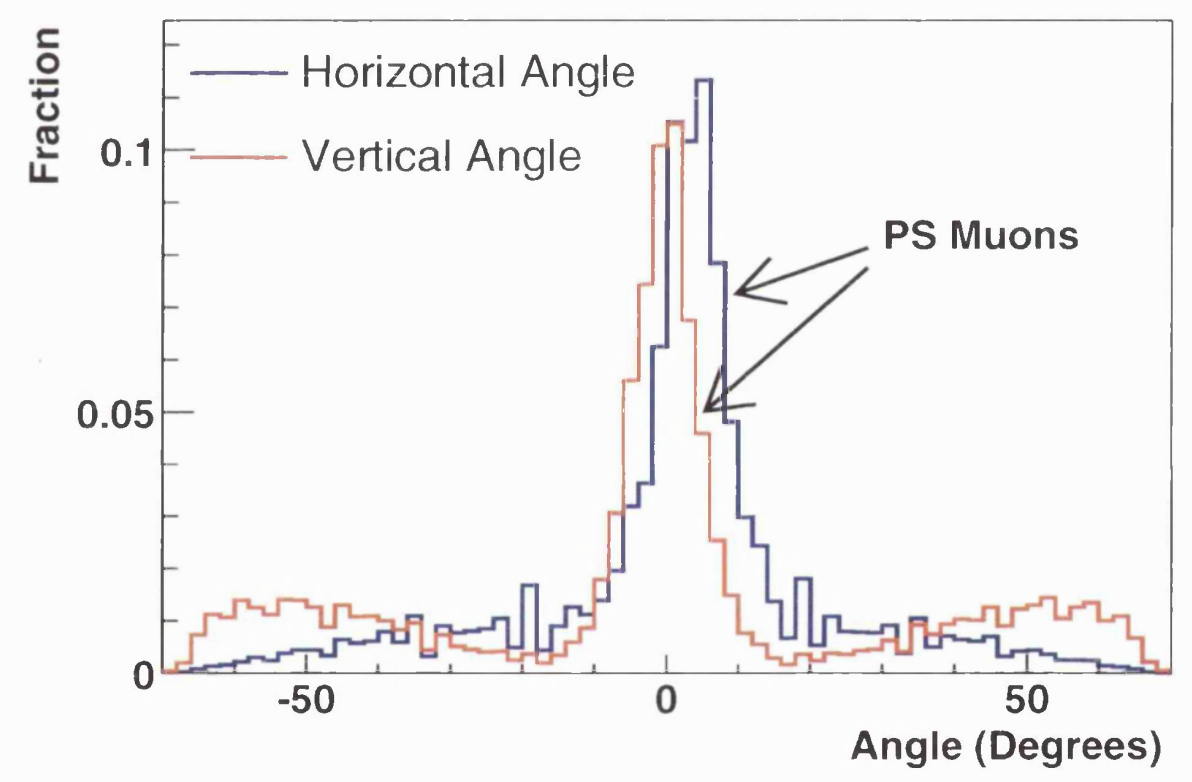

Figure 7.40: The angular distribution of muons in the T7 beam enclosure, when the local beam stop for the $T 7$ is in place. The peak at small angles, measured with respect to the beam line, is due to PS muons. These are high energy muons that traverse the entire length of the CalDet.

Due to the small angles with respect to the beamline, at which the PS muons are travelling through the detector, the need for a path length correction is greatly reduced. The correction described in Equation 7.21 is sufficient to remove the small path length dependence. 


\section{Zero Reconstruction}

There are far fewer corner clipping PS muons than there are corner clipping cosmic ray muons, making zero reconstruction easier. As with cosmic muons the scintillator light output was simulated for a sample of muons with the same angular distribution as the PS muons. The fraction of zeros as a function of light output from the simulation is compared to the number of zeros determined using single ended hits in the CalDet data, this is shown in Figure 7.41. Unlike the cosmic data, the simulation predicts fewer zeros than are observed looking at single sided hits in the detector. This could in part be due to deadtime in the electronics; if a PMT has recently been read out (either from a previous event or noise hit) then those electronics channels can appear to be 'dead' when the muon passes through the attached scintillator strip. If the electronics on one side of a scintillator strip are 'dead' when a muon passes through that strip then the number of zeros will be over estimated using single sided hits. In both simulation and data the fraction of zeros is the same for both even and odd planes. The curve is not an exponential as one might expect from Poisson statistics, this is due to the fact that the muon spectra are not fully Poissonian due to the contributions from a range of angles and the Landau tail of the muon energy deposits.

\section{Energy Loss}

One disadvantage of using the PS muons, compared to cosmics, is that all of the muons are travelling the same way through the detector. Therefore the particles are at their most energetic, and therefore deposit the most energy, at the front of the detector. Assuming the PS muons are below $\sim 5 \mathrm{GeV}$, there should be a non-negligible slope to the energy deposited as a function of plane, as shown in Figure 7.42. The $3 \%$ slope observed implies an energy of $\sim 3 \mathrm{GeV}$, from comparison with simulated data. This slope must be removed from the PS muon calibration constants, to ensure a bias between the front and back of the detectors is not introduced. 
Comparison of Zero Fraction from Simulation and Data (PS Muons)

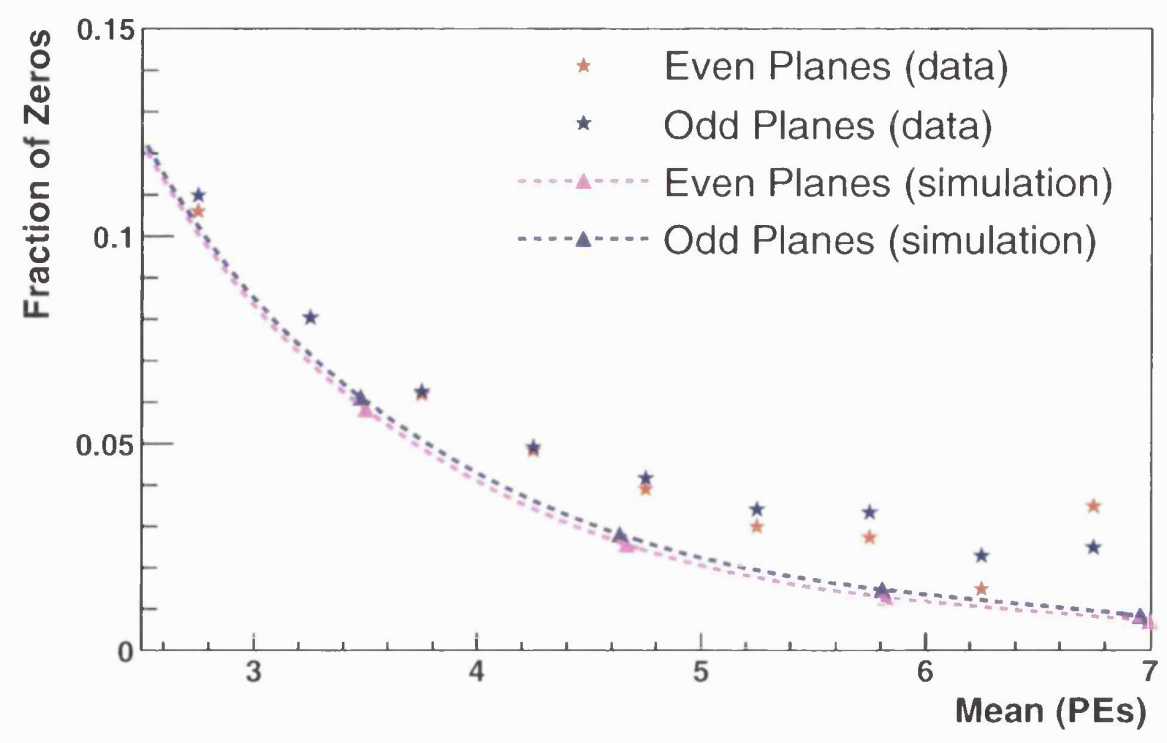

Figure 7.41: The fraction of zero photoelectron hits as a function of light level for PS muons. The results from the simulation, using the PS muon angular distribution, predict less zeros than are observed looking at single ended hits in the data. In both the simulation and the data the number of zeros in even and odd planes agree.

\section{Performance of PS Muon Calibration Constants in the T7}

These calibration constants have been tested using a sample of $1.8 \mathrm{GeV}$ stopping beam muons. The total energy deposited per plane of scintillator, in cosmic MEU, is shown in Figure 7.43. A straight line is fitted between planes 1 and 39, the width of the fractional residual distribution is less than $2 \%$. An even-odd bias is not introduced using these constants, and the anomalous plane 35 is well calibrated. 


\section{Total Energy Deposited per Plane by PS Muons}

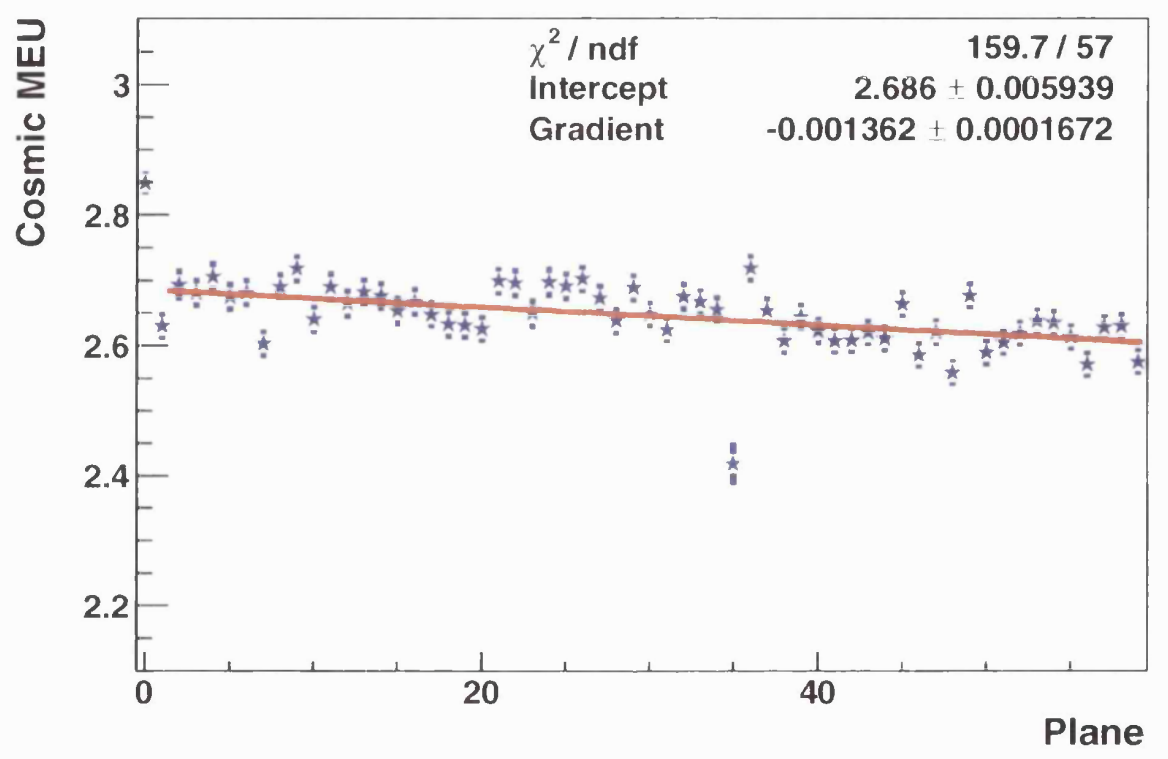

Figure 7.42: The total energy deposited per plane, in terms of cosmic MEU, for PS muons. There is a slope due to the energy loss of the muons as they traverse the detector from front to back. The slope implies a significant fraction of the PS muons are below $5 \mathrm{GeV}$ when they enter the detector, above this energy the slope due to energy loss is negligible. 

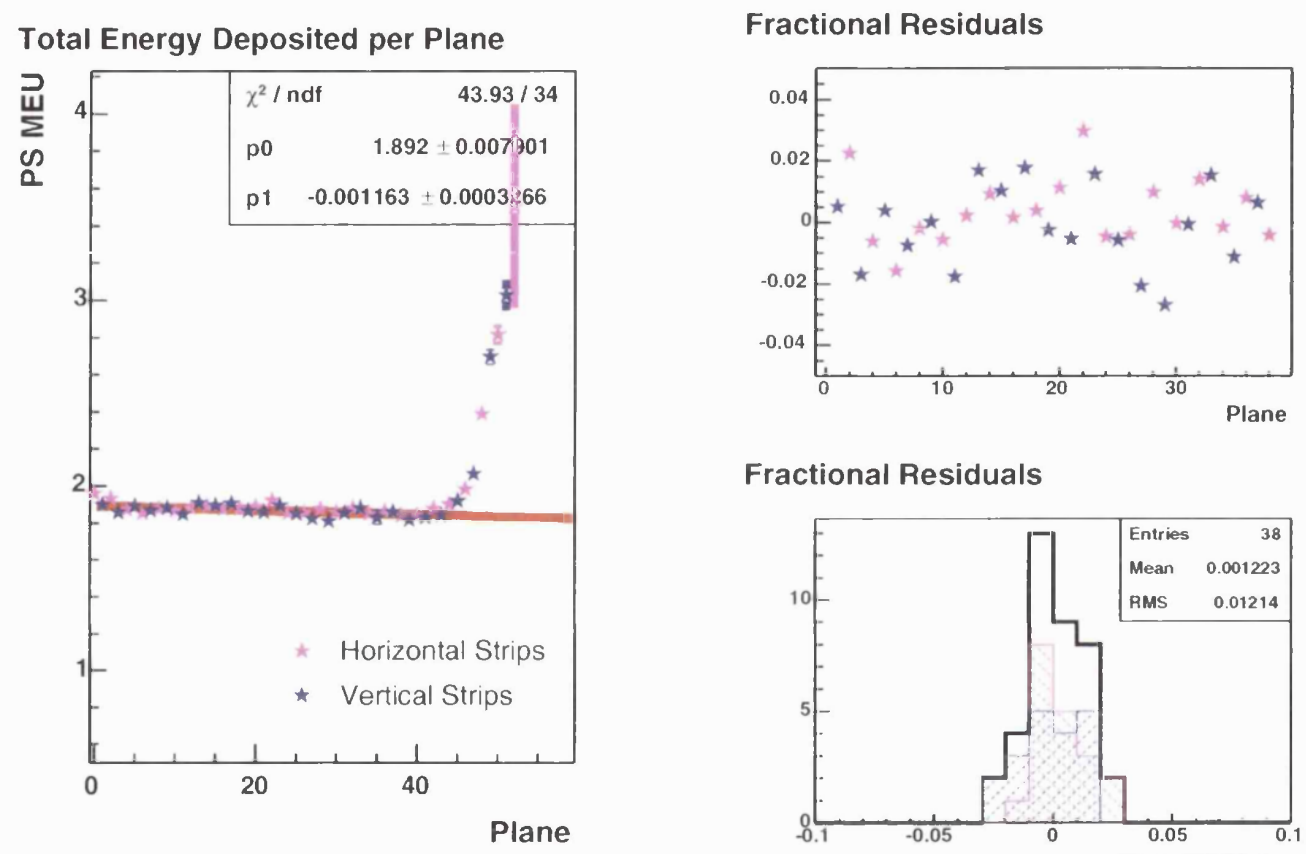

\section{Fractional Residuals}

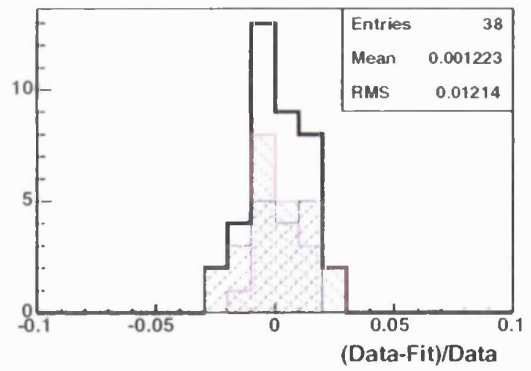

Figure 7.43: The performance of PS muon calibration constants in the T7. The total energy deposited per scintillator plane by a sample of $1.8 \mathrm{GeV}$ stopping beam muons, in PS MEU, is shown. Due to the very similar horizontal and vertical angular distribution of PS muons, this calibration does not introduce a noticeable even-odd asymmetry in the residual plots. The total width of the residual histogram is less than $2 \%$, compared to the $12 \%$ width of the residuals in raw uncalibrated ADC units. 


\subsubsection{Calibration in the T11 Testbeam}

The CalDet has been exposed to two separate testbeams at CERN: the T7 and T11 testbeams. The T11 beam is optimized for lower energies than the T7 beam, and as such a number of measurements have been carried out using the T11 beam that would not be possible with the Tr beam. When the detector is moved from one testbeam area to another, it is completely de-cabled and craned from one location to the other. The light output of each scintillator will be slightly different in the new location, particularly due to the differing optical connections. Therefore, the calibration constants derived in the $\mathrm{T} 7$ area can not be transferred to the $\mathrm{T} 11$, instead new calibration constants must be derived. The T11 testbeam is angled approximately $30^{\circ}$ from the T7 testbeam, meaning that the PS muons do not travel through the detector parallel to the beam. It is not possible to fully calibrate the detector using PS muons in the T11, as they do not have full coverage of the detector, as illustrated in Figure 7.44. This leaves cosmic muons as the only possible source for a strip to strip calibration.

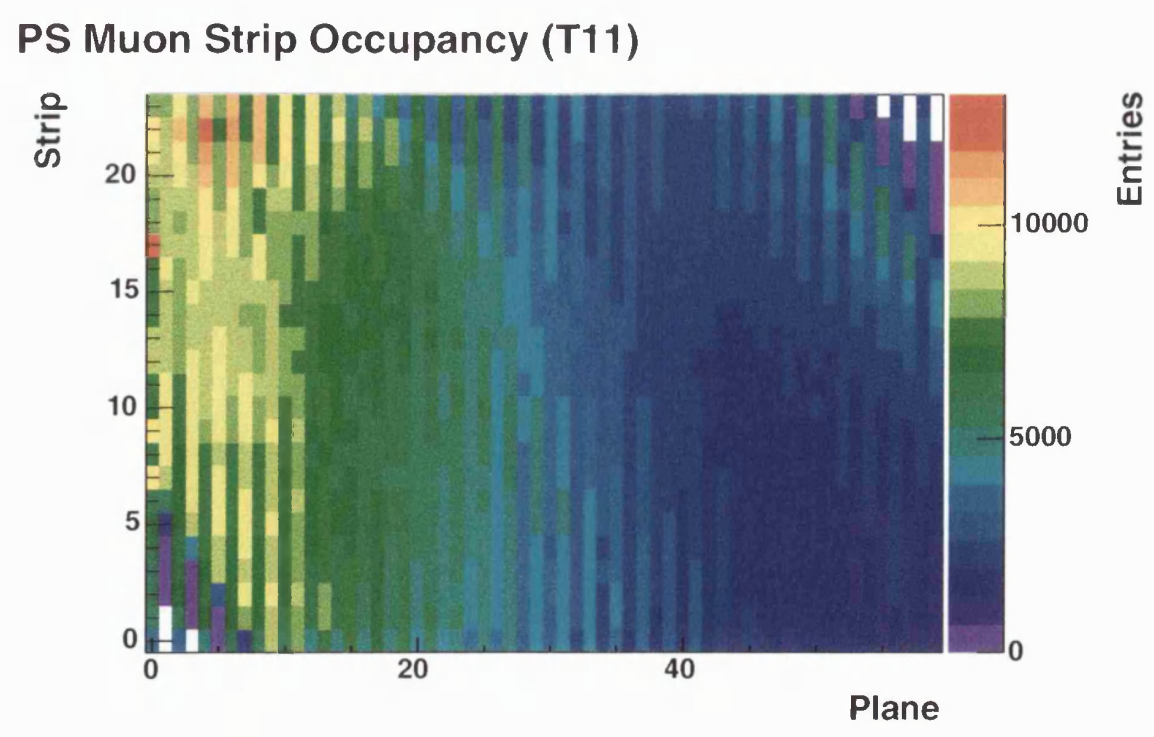

Figure 7.44: The strip occupancy of PS muons in the T11 testbeam area. PS muons in the T11 are not suitable for fully calibrating the detector as only the front half of the detector is able to obtain sufficient statistics for calibration. 


\subsection{Stopping Muons}

A relative calibration is needed to relate muon energy units from the CalDet in the T7 beamline, to the MEU from the CalDet in the T11 beamline. Stopping beam muons are used to achieve this calibration. The stopping muons are more likely to undergo multiple scattering in the detector, therefore the straight line cosmic tracking algorithm is not suitable. Instead, the beam muons are tracked using the algorithm described in $[56]$ and Figure 7.45 shows an example event display of a $1.8 \mathrm{GeV}$ muon.
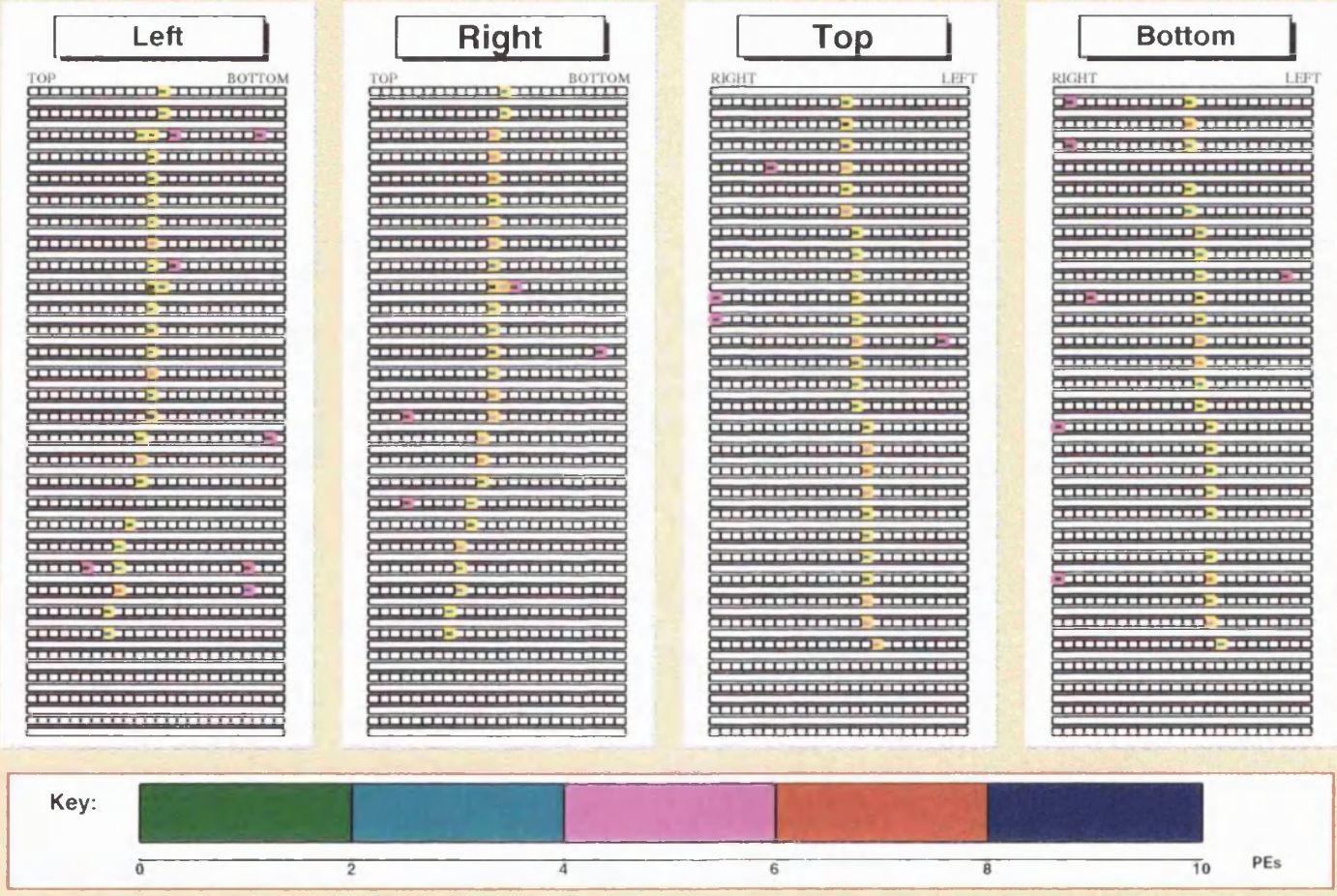

Figure 7.45: An event display showing a $1.8 \mathrm{GeV}$ beam muon from the CalDet. The muon stops after travelling though 50 planes of steel. 'Left' and 'Right' refer to the ends of the horizontal strips, 'Top' and 'Bottom' to the ends of the vertical strips. The hits highlighted in yellow are those which are included in the muon track, by the muon tracking algorithm described in [56].

The first step in the relative calibration procedure is to select a sample of stopping muons. Beam muons are formed predominantly from the decay of pions, which 
are produced from proton interactions with the target. These pions can decay at any point between the target and the detector, resulting in a polychromatic muon energy distribution, due to the decay kinematics of the pions, as illustrated in Figure 7.46. The range of beam muons, in centimetres of scintillator, is plotted for a run with nominal energy of $1.8 \mathrm{GeV}$. The peak at $51 \mathrm{~cm}$ is due to muons with the nominal energy, the distribution below this consists of lower energy muons, and pions which do not have a large hadronic shower. A sample of monochromatic muons is selected using a range cut around the nominal range peak.

\section{Range of Stopping Beam Muons (from 1.8 GeV Run)}

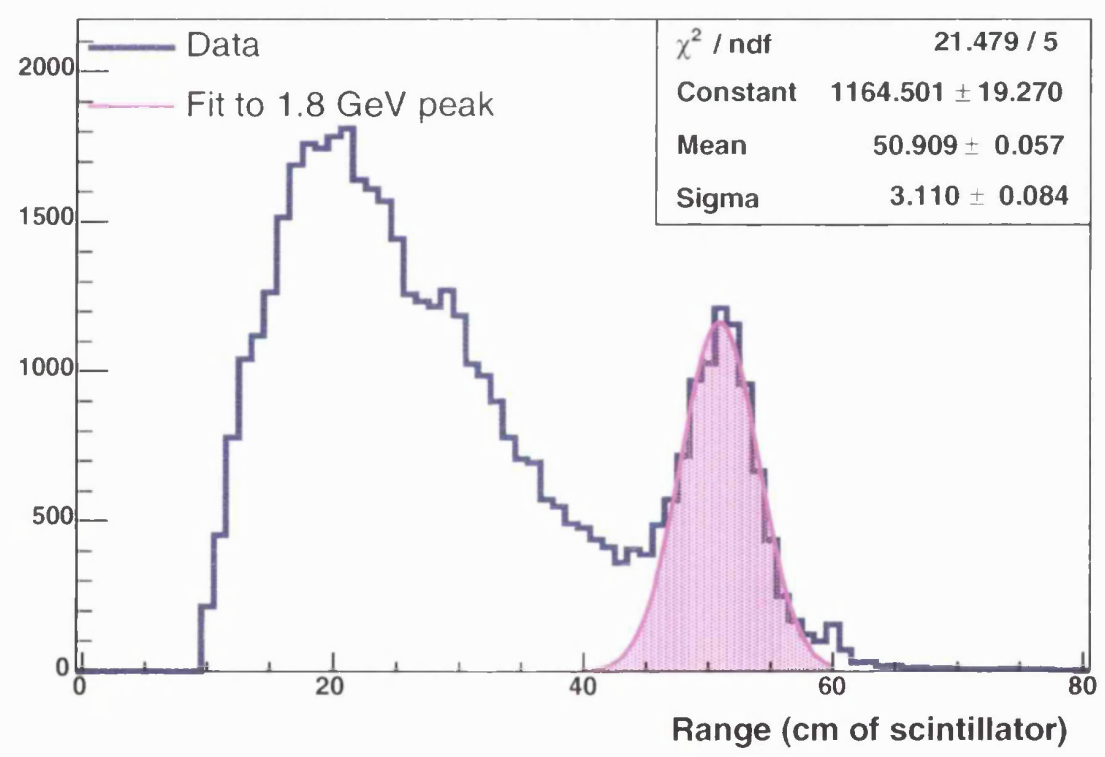

Figure 7.46: The range of beam muons, in cm of scintillator, is shown for a run with the magnets set to $1.8 \mathrm{GeV}$. The muon energy spectrum is not monochromatic, due to the decay kinematics of the pions from which the majority of muons are produced. The fitted peak centred around $51 \mathrm{~cm}$ is from muons with the nominal beam energy of $1.8 \mathrm{GeV}$. Some fraction of the shorter range particles are not muons, but instead are pions which have not produced a large hadronic shower. These pions which do not produce a significant shower in the detector are almost indistinguishable from the off momentum muons and are tagged as muons by the tracking algorithm.

This procedure is repeated to select stopping muons for all beam energies be- 
tween $0.8 \mathrm{GeV}$ and $2.0 \mathrm{GeV}$, below $0.8 \mathrm{GeV}$ the muons leave short tracks that are hard to distinguish from other particles and above $2.0 \mathrm{GeV}$ the muons do not stop in the detector. From the T11, samples of stopping muons were obtained at 0.8, 1.0, $1.2,1.4,1.6,1.8$ and $2.0 \mathrm{GeV}$ and in the $\mathrm{T} 7$, which is designed for higher energies, stopping muons were only obtained at $1.0,1.8$ and $2.0 \mathrm{GeV}$.

The total energy deposited by beam muons summed over the full length of the muon track, in MEU, as a function of range can then be plotted from both the T7 and T11 testbeams, as in Figure 7.47. In these plots the strip to strip calibrations in the T7 are derived from PS muons and those in T11 from cosmic muons, to accentuate the difference in slopes between the two beamlines. The discrete jumps in total energy come from the discrete steps in beam energy, as the uncertainty on muon range due to straggling is greater than the uncertainty in beam momentum. If smaller steps in beam energy were taken, then these jumps would be washed out. Using the slopes of the two fitted lines it is possible to calibrate the effect of the differing muon samples used to construct the strip to strip calibration constants. The energy scale can be set to a logical muon energy unit, such that a muon which travels through $50 \mathrm{~cm}$ of scintillator, deposits $50 \mathrm{MEU}$ in the detector. 


\section{Total Muon Energy Deposited v Range (T11)}

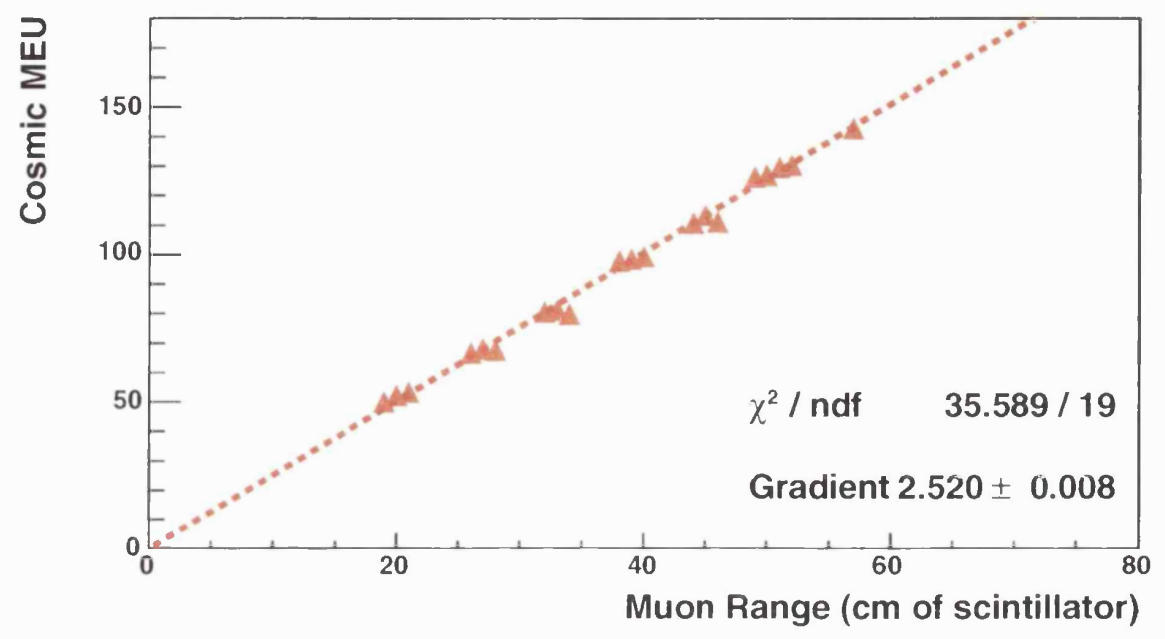

\section{Total Muon Energy Deposited v Range (T7)}

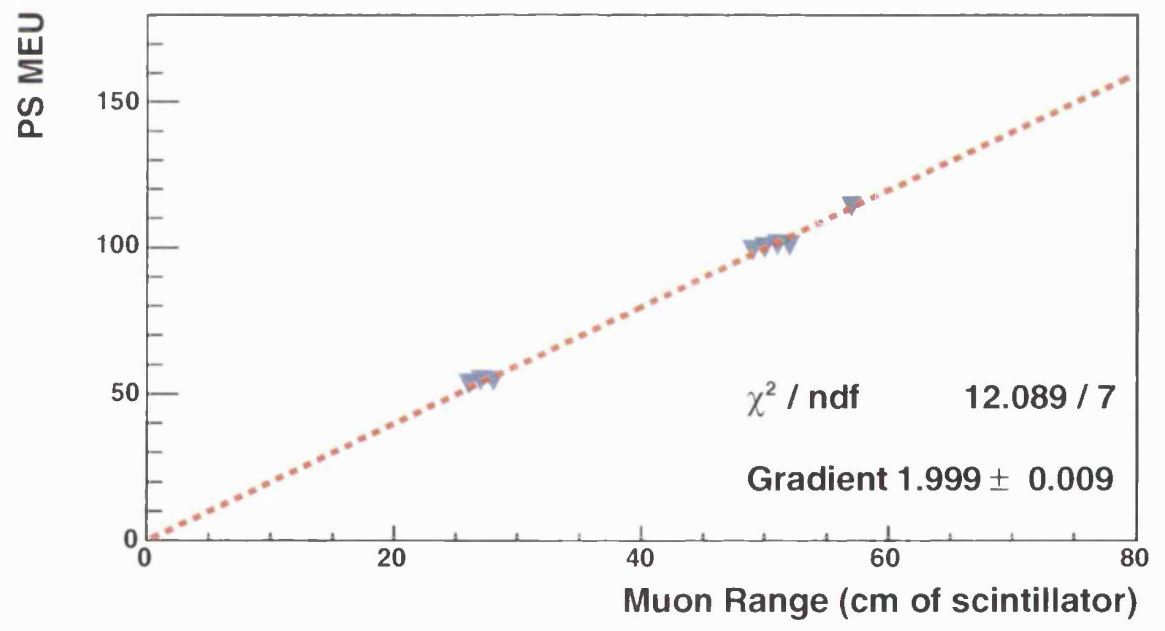

Figure 7.47: The total energy, in arbitrary MEU, deposited by beam muons as a function of range, in cm of scintillator, is plotted for beam muons from the T11 and the T7. The different slopes are the result of the different muon samples used to construct the calibration constants, cosmic muons were used in T11 and PS muons in T7. Using these two lines it is possible to calibrate the energy scale of the CalDet in the two beam locations, both are scaled such that a muon travelling through $50 \mathrm{~cm}$ of scintillator deposits $50 \mathrm{MEU}$. 


\subsection{Results of Calibration}

To thoroughly test the calibration, energy deposits in the scintillator strips must be used. $1 \mathrm{GeV}$ electrons have been used to test the drift point correction, the strip to strip calibration and the relative calibration between the T7 and T11 detectors.

\section{Drift Point Correction}

In order to test the performance of the drift point correction, the high voltages applied to the PMTs were changed and some $1 \mathrm{GeV}$ electron data was obtained. On half of the PMTs the high voltage was increased by $25 \mathrm{~V}$, on the other half the high voltage was decreased by the same amount. These high voltage changes resulted in the PMT gains changing by up to $\sim 40 \%$.

The results of this test are shown in Figure 7.48, where the total energy deposited by the electrons is shown in terms of ADC, drift corrected ADC and cosmic MEU, with each sample of PMTs shown separately. The ADC distributions show a $\sim 60 \%$ difference in mean, from a restricted range Gaussian fit, between those PMTs whose voltage was raised, and those whose voltage was decreased. The means of the drift corrected ADC distributions differ by $\sim 2 \%$, most of this difference is removed in the MEU distributions, the means of which agree at the $0.5 \%$ level. The means of the distributions are measured using restricted Gaussian fits to minimize the effect of the tails of the distributions. The shape of these distributions is discussed in more detail in [72].

\section{Strip to Strip Calibration}

The Calibration Detector has an unusual readout cable configuration; half of the readout cables are $6 \mathrm{~m}$ long clear fibre cables, the other half are $4 \mathrm{~m}$ long green wavelength shifting fibres. This results in $\sim 30 \%$ more light from those strip-ends read out with the clear fibres, due to the difference in attenuation. Histograms of the T7 PS muon strip to strip calibration constants, for each of the four views, are shown in Figure 7.49. 


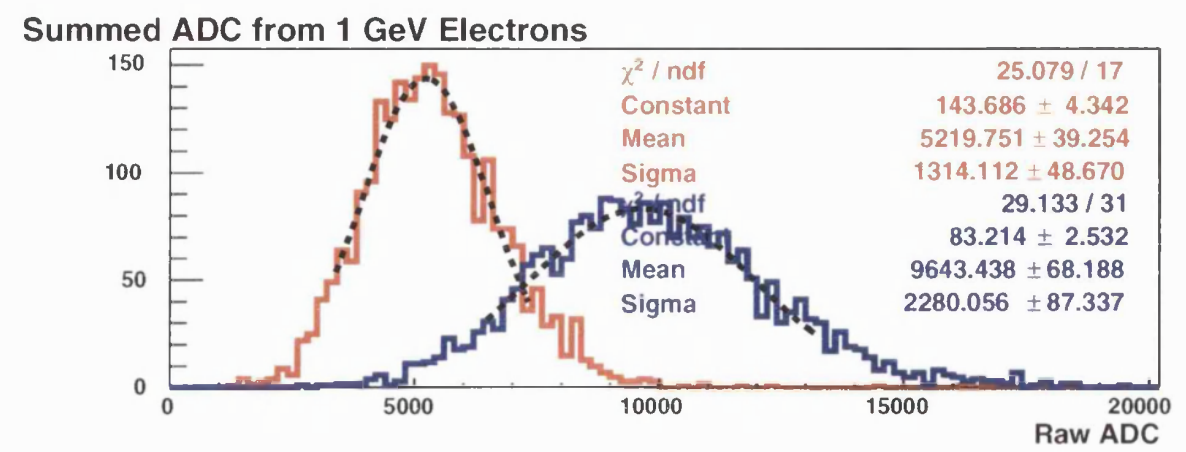

Summed Corrected ADC from $1 \mathrm{GeV}$ Electrons

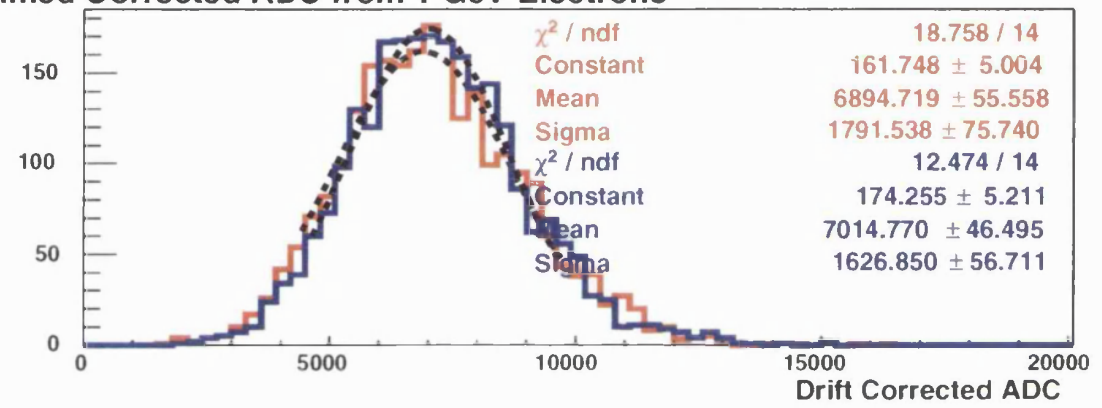

Summed MEU from $1 \mathrm{GeV}$ Electrons

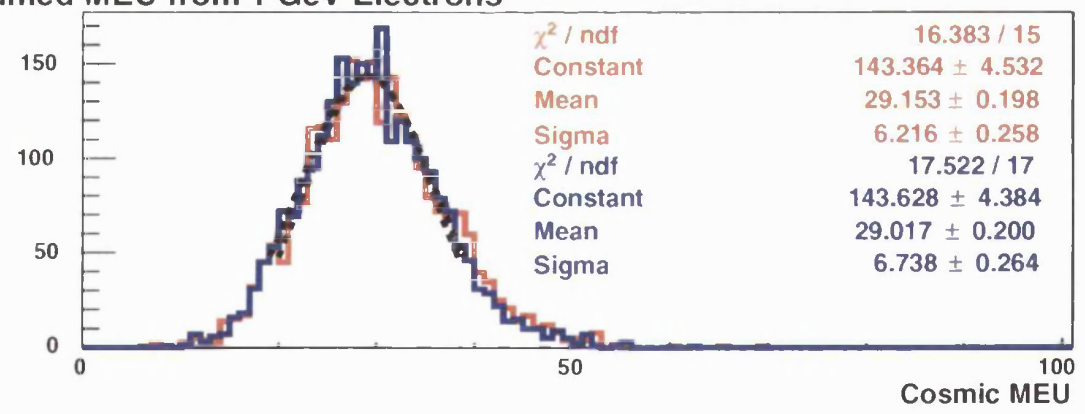

Figure 7.48: The total energy deposited by $1 \mathrm{GeV}$ electrons in the CalDet, after the high voltage on all of the PMTs were changed by $25 \mathrm{~V}$. Half of the PMTs had their high voltage increased, the other half had their high voltage decreased. The top plot shows the effect of these voltage changes, the red histogram is the summed ADC from the PMTs whose voltages were decreased and the blue histogram the same for the PMTs with increased voltages. The middle plot shows the same distributions in terms of drift corrected ADC, and the bottom plot is in drift corrected cosmic MEU. The $\sim 60 \%$ difference in ADC is corrected to $\sim 0.5 \%$ in $\mathrm{MEU}$. 

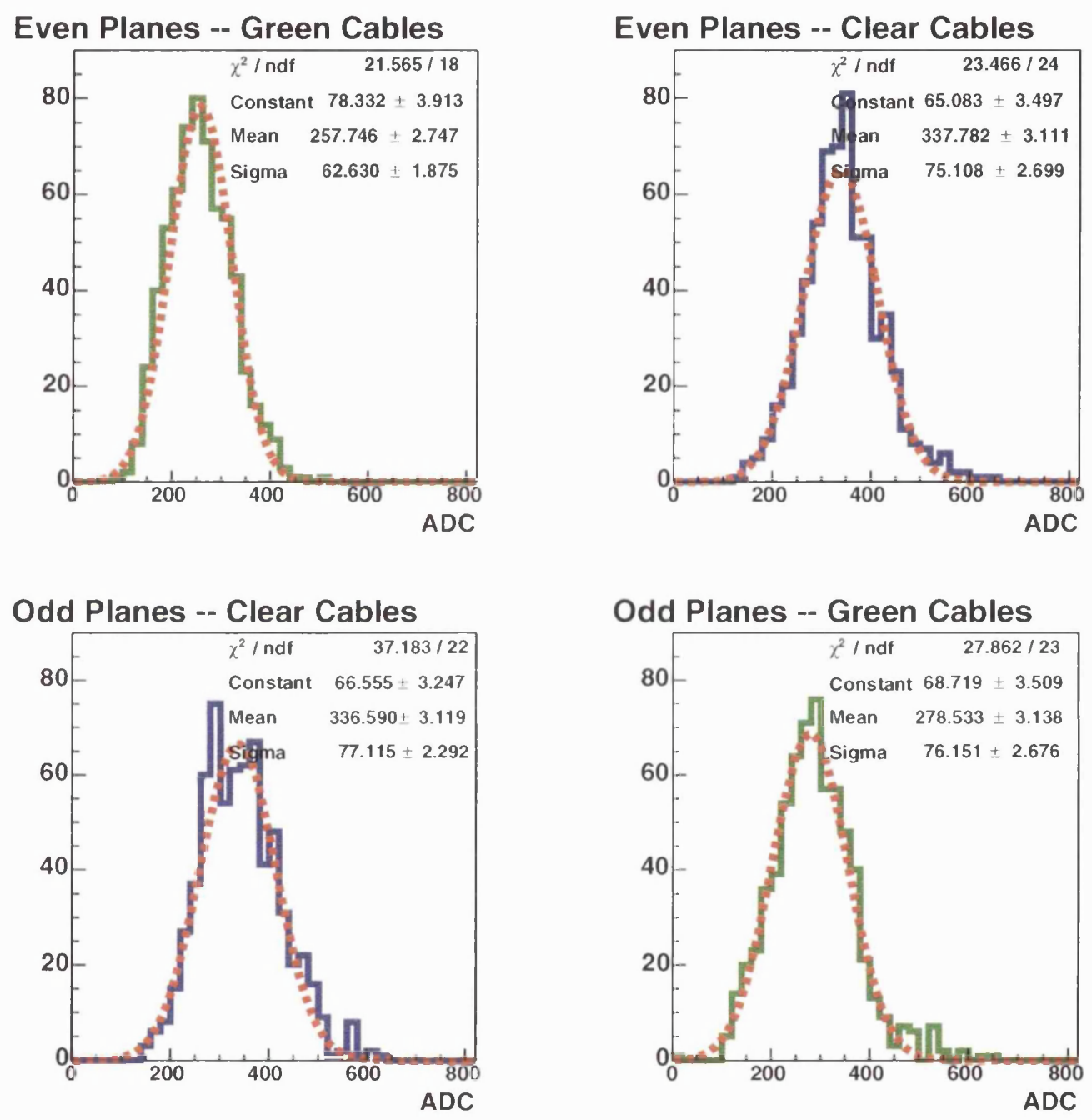

Figure 7.49: Histograms of the PS muon strip to strip calibration constants from the T7. The four views are plotted separately. The green histograms consist of those strip-ends read out using green wavelength shifting fibre cables, the blue histograms are those stripends read out using clear fibre cables. There is $\sim 30 \%$ more light output from the clear fibre strip-ends than the green fibre strip-ends. Other non-uniformities and errors on the calibration constants contribute to the width of the individual distributions. 
The difference in strip-end response due to the readout cables provides a good test of the strip to strip calibration. A sample of $1 \mathrm{GeV}$ electrons was selected from the T7 testbeam using the threshold Čerenkov detectors. The total energy seen in the detector is plotted separately for the green and clear readout cables in Figure 7.50. The top plot shows the total energy in raw ADC. Calculating the means of the green and clear summed ADC distributions using a restricted range Gaussian fit, the clear distribution has $\sim 13 \%$ more charge than the green. The bottom plot shows the total energy after strip to strip calibration in terms of PS MEU. The means of the two distributions, from restricted Gaussian fits, differ by less than $0.1 \%$.

\section{Relative Calibration between T7 and T11}

The relative calibration, achieved using stopping muons, between the T7 and T11 detector configurations has been tested. A sample of $1 \mathrm{GeV}$ electrons has been selected from each testbeam using the threshold Črenkovs provided. The total energy deposited by these electrons is compared before and after the relative calibration is applied in Figure 7.51. Before the relative calibration the responses differ by $32 \%$ due to the different samples of muons used to determine the calibration constants in each location. This difference is calibrated to better than $2 \%$ after the relative calibration is applied. Some of this difference could be due to uncertainties in the testbeam momenta. 


\section{Total ADC for $1 \mathrm{GeV}$ Electrons}

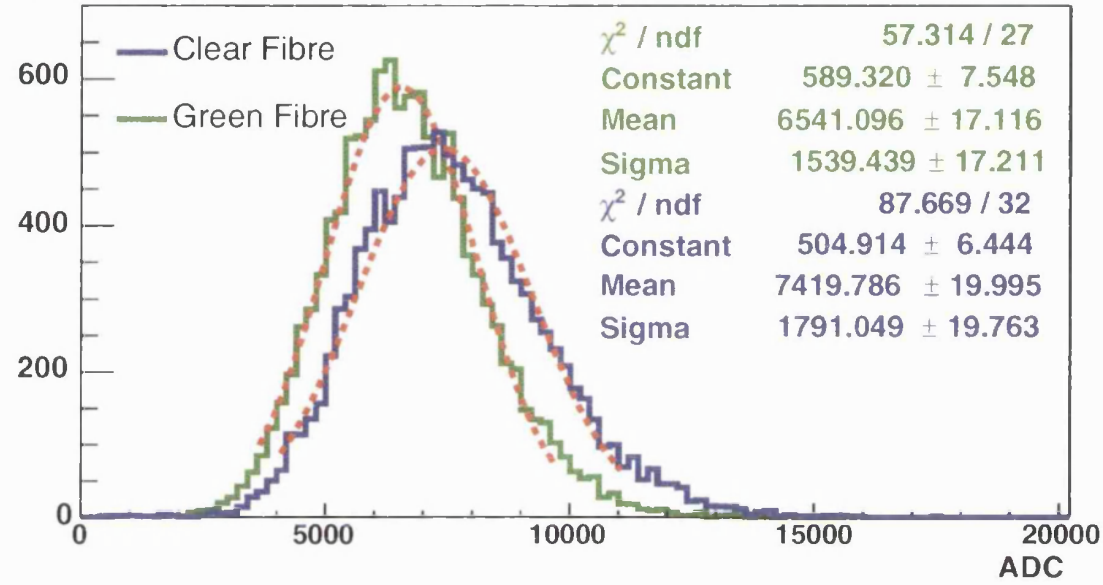

Total MEU for $1 \mathrm{GeV}$ Electrons

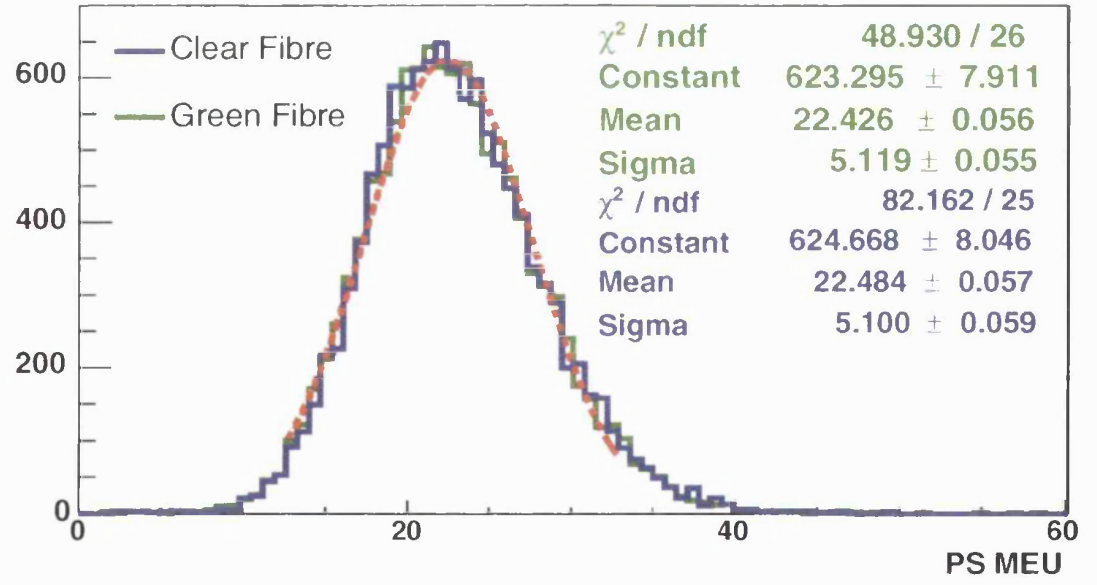

Figure 7.50: The total energy deposited by $1 \mathrm{GeV}$ electrons, plotted separately for the green and clear fibre optical readout cables. The top plot shows an $\sim 13 \%$ difference in the response in terms of raw, uncalibrated, ADC. After the PS muon strip to strip calibration is applied the means of the two distributions, taken from restricted range Gaussian fits, agree to within $0.1 \%$. 


\section{Total MEU for $1 \mathrm{GeV}$ Electrons}

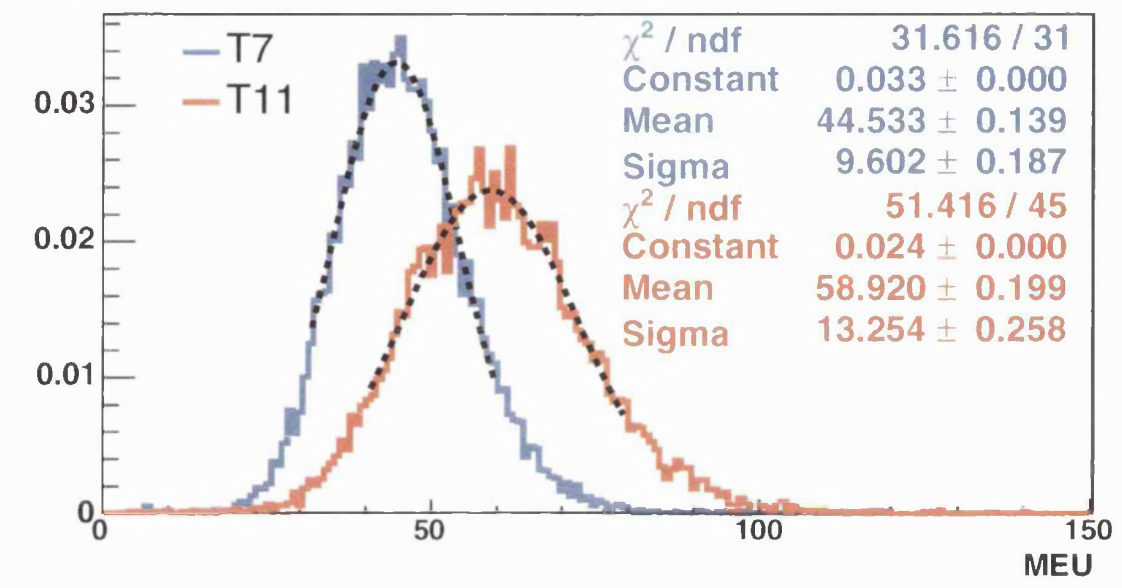

Total MEU for $1 \mathrm{GeV}$ Electrons

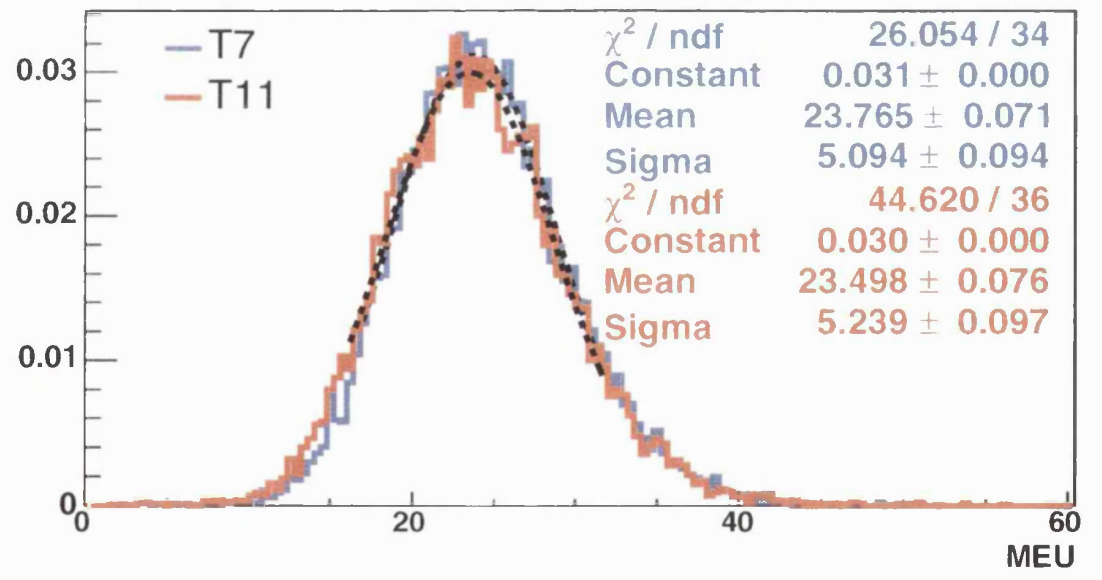

Figure 7.51: The total energy deposited by $1 \mathrm{GeV}$ electrons from the T7 and T11 testbeams, before and after relative calibration using stopping beam muons. The $32 \%$ discrepancy between the two beamlines before relative calibration stems from the sample of muons used to define the muon energy units; in the T7, the total energy deposited per plane by PS muons was used and in the T11, corrected cosmic ray muons were used. After the relative calibration is applied the discrepancy is reduced to less than $2 \%$. 


\subsection{Summary}

Techniques for calibrating the MINOS detectors, using muons and the light injection system, have been developed and tested at the Calibration Detector. One of the goals for the MINOS calibration is to achieve a relative calibration of the energy scales of the Near and Far Detectors to $2 \%$.

The light injection drift point correction ensures the response of individual PMT and electronics channels does not vary with time. Variations in gain of up to $40 \%$ have been calibrated to the $1 \%$ level. This has been shown using both the light injection system itself and energy deposits from $1 \mathrm{GeV}$ electrons.

The muon strip to strip calibration is used to remove variations in the light output of scintillator strips and the optical path from the strips to the PMTs. The removal of these variations allows energy deposits in different strips to be compared directly. Variations in strip-end response of up to $\sim 30 \%$, from the different readout cables, have been calibrated to better than $2 \%$ using strip to strip calibration constants derived from muon energy deposits. This has been demonstrated using electrons and muons from the beam.

The final stage of the relative calibration is a cross calibration between the detector in its $\mathrm{T} 7$ and $\mathrm{T} 11$ incarnations, thus ensuring that particles from the two beams can be compared. This calibration has been achieved using stopping beam muons. The calibration has been shown to work at the $2 \%$ level using electron events from the two beams. 


\section{Chapter 8}

\section{Cross-Detector Calibration}

The main MINOS measurement of the neutrino oscillation parameters will involve a comparison of the Near and Far Detector neutrino energy spectra, as discussed in section 4.3. Before this comparison can take place the two detectors must be calibrated relative to each other and also to the CalDet, enabling an absolute calibration of the energy scale in terms of GeV. Stopping muons will be employed for the relative calibration using a technique similar to that described in section 7.3 for the relative calibration of the CalDet between the T7 and T11 beamlines. In this chapter the first cross-detector calibration between the Far Detector and the Calibration Detector will be discussed.

\subsection{Strip to Strip Calibration of the Far Detector}

Before a cross-detector calibration can be investigated a strip to strip calibration of the Far Detector using cosmic ray muons must be performed. This calibration removes any variance in the response of the strips within the Far Detector, such that all strips may be considered in the cross-detector calibration. At the time of writing only the first of the two super-modules had been operational long enough to collect adequate statistics for a strip to strip calibration.

The Far Detector does not suffer from the same difficulties regarding angular asymmetry as the Calibration Detector, because the Far Detector scintillator strips 
are all at a $45^{\circ}$ angle to the horizontal. Figure 8.1 shows the angular distribution of muons used in the strip to strip calibration of the Far Detector. As with Figure 7.27 the angular distribution is plotted both in terms of the horizontal and vertical angles and of the natural angles measured by the Far Detector scintillator strips. The angular asymmetry visible between positive and negative angles is the result of differences in the rock overburden above the detector. This asymmetry is not a problem for the strip to strip calibration as path length through the scintillator strip does not depend on the sign of the angle.

The muon angular spectrum at the Far Detector is very similar for both the ' $U$ ' and ' $V$ ' strips. For this reason a path length correction is not strictly necessary, as the distribution of path lengths for each strip in the detector is similar. The only correction necessary for a strip to strip calibration is a zero reconstruction, to ensure that a bias is not introduced between the high and low light level strips. The iterative correction described in section 7.2.1 should be directly applicable to the Far Detector cosmic muons. However for the purpose of illustrating the viability of a cross-detector calibration, zero reconstruction using single sided hits is sufficient. This correction is imperfect, but does allow a cosmic MEU scale to be defined for the Far Detector using lower statistic muon samples. 


\section{Angular Distribution of Cosmic Ray Muons in the Far Detector}

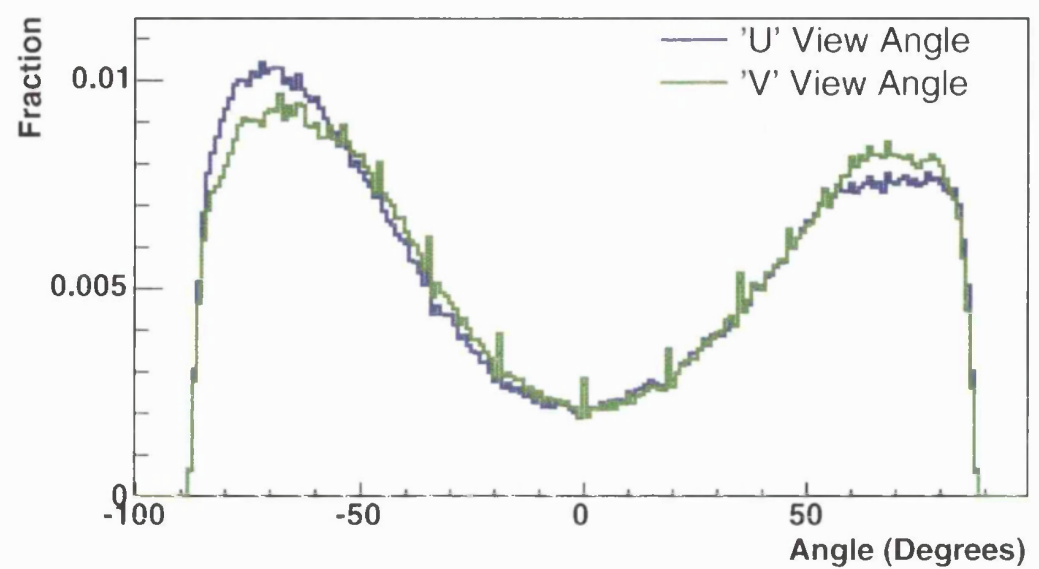

Angular Distribution using Transformed Angles

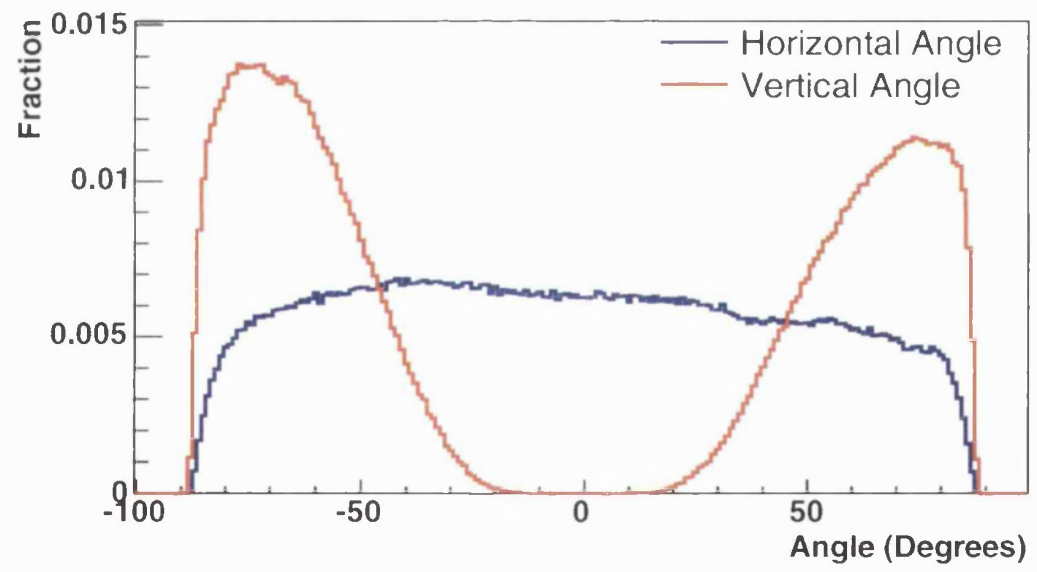

Figure 8.1: The angular distribution of cosmic ray muons at the Far Detector. The top plot shows the angular distribution in terms of the natural angles measured by the Far Detector scintillator strips. The spikes are due to the finite angular resolution obtained using the $4.1 \mathrm{~cm}$ wide strips. The bottom plot shows the large angular asymmetry in terms of the horizontal and vertical angles, which would be the natural angles if the strips were oriented as in the CalDet. The asymmetry between positive and negative angles, in the ' $\mathrm{U}$ ' and ' $\mathrm{V}$ ' views, is due to differences in the rock overburden above the detector. This asymmetry does not introduce a bias in the calibration as the path length distributions depend on $|\tan \theta|$, see Equation 7.21. 


\subsection{Stopping Muons in the Far Detector}

A sufficient sample of stopping muons in the Far Detector is needed for comparison with the stopping beam muons in the Calibration Detector. Any sample of muons for which the range is known, i.e. muons that do not exit the detector, can be used for the calibration. The following cuts were used to select a sample of stopping muons in the detector:

i) There must be a track in the event

ii) The number of hit strips $>10$

iii) The track end point $>1 \mathrm{~m}$ from side of detector

iv) The track end point $>1 \mathrm{~m}$ from end of detector

v) The track start point $<0.3 \mathrm{~m}$ from detector edge

vi) The entire track must be in the first super-module.

vii) Over $95 \%$ of planes along the track must not contain a shower.

Cuts iii) and iv) ensure the track stops in the detector and cut v) removes any contained atmospheric neutrino events from the sample.

The effect of these cuts is shown in Figure 8.2. After the cuts are applied a sample of 3488 stopping muons are selected from an initial sample of 6776311 events collected in 35 days of livetime from a two month period. Of those events containing a track $\sim 0.6 \%$ are non showering stopping muons.

Figure 8.3 shows an event display of a stopping cosmic ray muon in the Far Detector. The muon enters the top of the detector and stops approximately two metres from the edge of the detector. The track is shown in terms of the conventional Cartesian coordinates $x, y$ and $z$; with $z$ lying along the beam direction and $x$ and $y$ in the horizontal and vertical directions respectively. Also shown are the track positions in terms of $u$ and $v$, as measured by the strips in the two orientations. 


\section{Selection of Stopping Muons in the Far Detector}

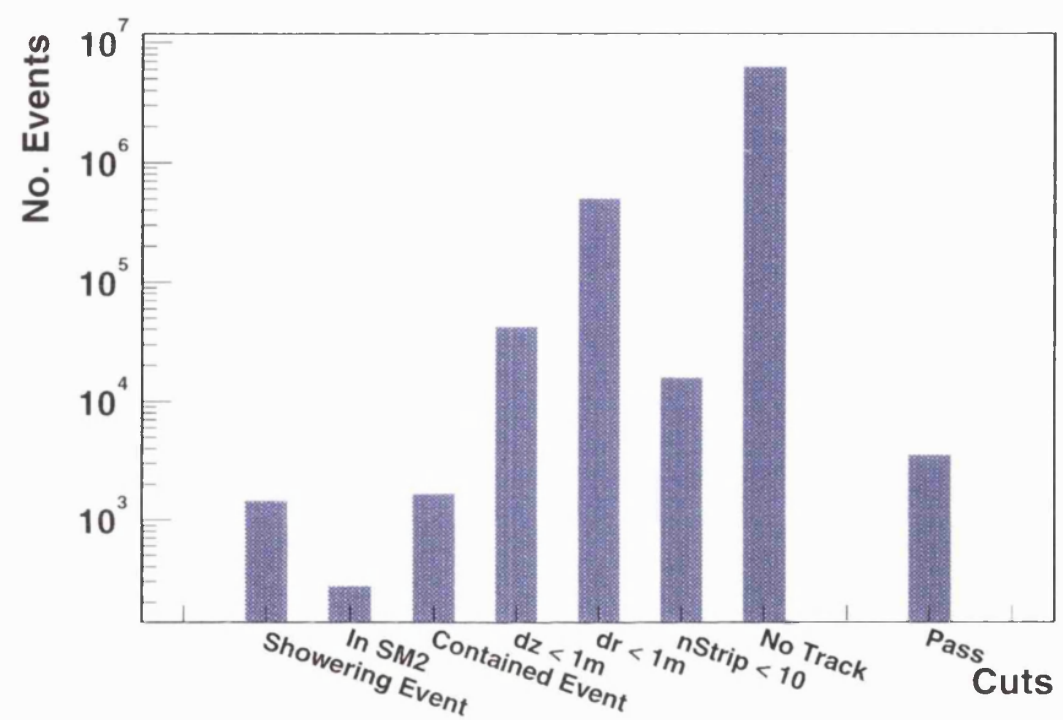

Figure 8.2: Cuts used to select stopping muons in the Far Detector. The cut labelled "In SM2" removes muons which enter the uncalibrated (at time of data collection) second super-module. The majority of events are short events which do not have a track. Of those events containing a track only $\sim 0.6 \%$ are stopping muons which pass all the cuts. The cuts which remove the most events are those which require the end of the track to be greater than $1 \mathrm{~m}$ from the edges of the detector. 

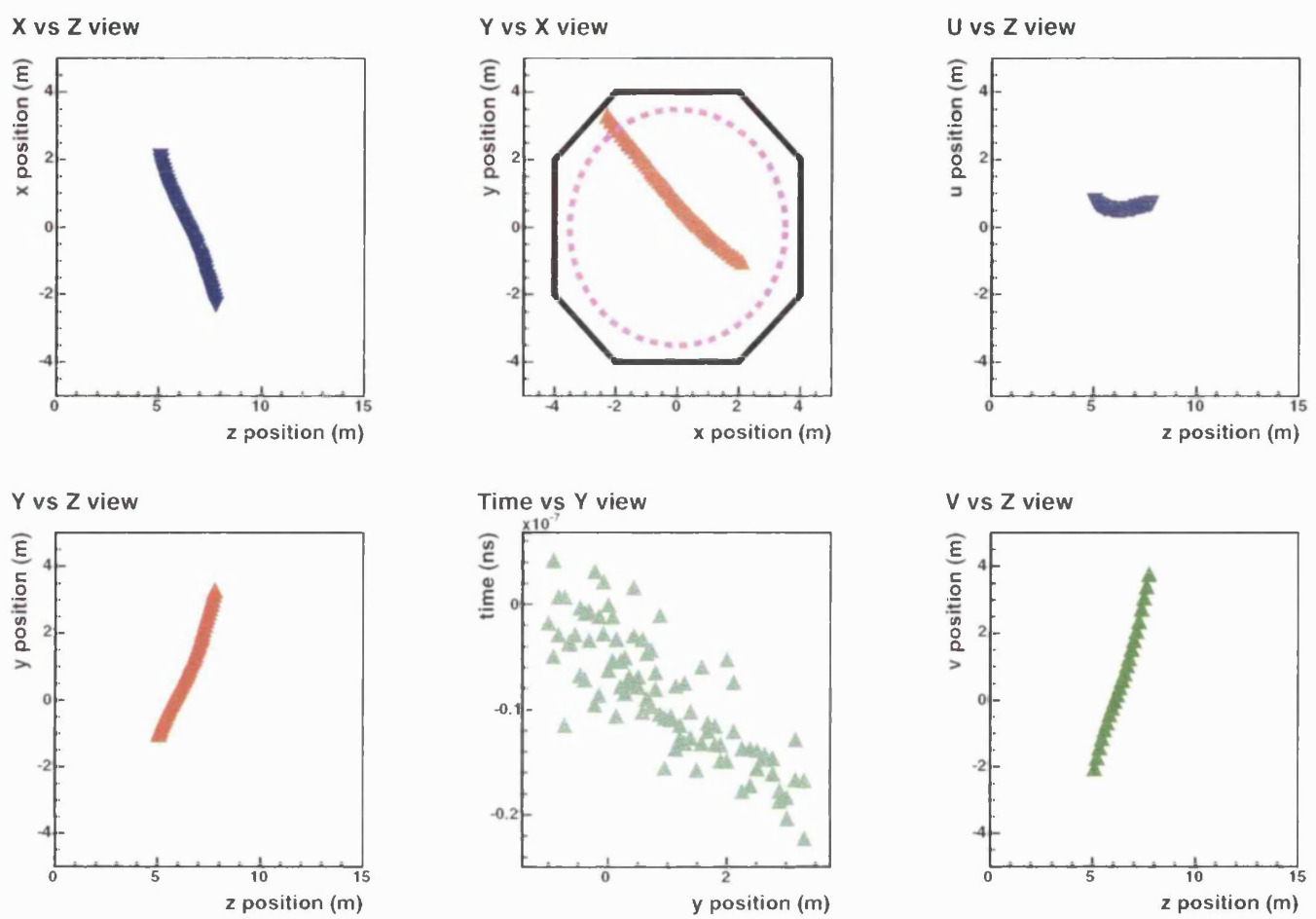

Figure 8.3: An event display showing a stopping cosmic ray muon in the Far Detector. The two leftmost plots show the tracked muon hits in terms of the transverse directions, $x$ and $y$, and the longitudinal direction, $z$. Where $z$ is defined as the direction of the beam and $x$ and $y$ measure the horizontal and vertical position. The two plots on the righthand side show the track in terms of the $u$ and $v$ directions, as determined by the strip orientations. The middle plot in the top row shows the track as a 2-dimensional projection into the $x-y$ plane, with the beam direction into the page. The black octagon shows the edges of the scintillator planes, and the dashed line the $1 \mathrm{~m}$ fiducial volume for containment of the muon end point. The plot in the middle of the bottom row shows time as a function of $y$ position. The track is clearly travelling from high to low values of $y$, i.e. the muon enters the top of the detector. 


\subsection{Cross-Detector Calibration}

To demonstrate that a relative calibration between the Calibration Detector and the Far Detector is viable, the total energy deposited by stopping muons as a function of range is plotted for both of the detectors in Figure 8.4.

\section{Total Muon Energy Deposited v Range}

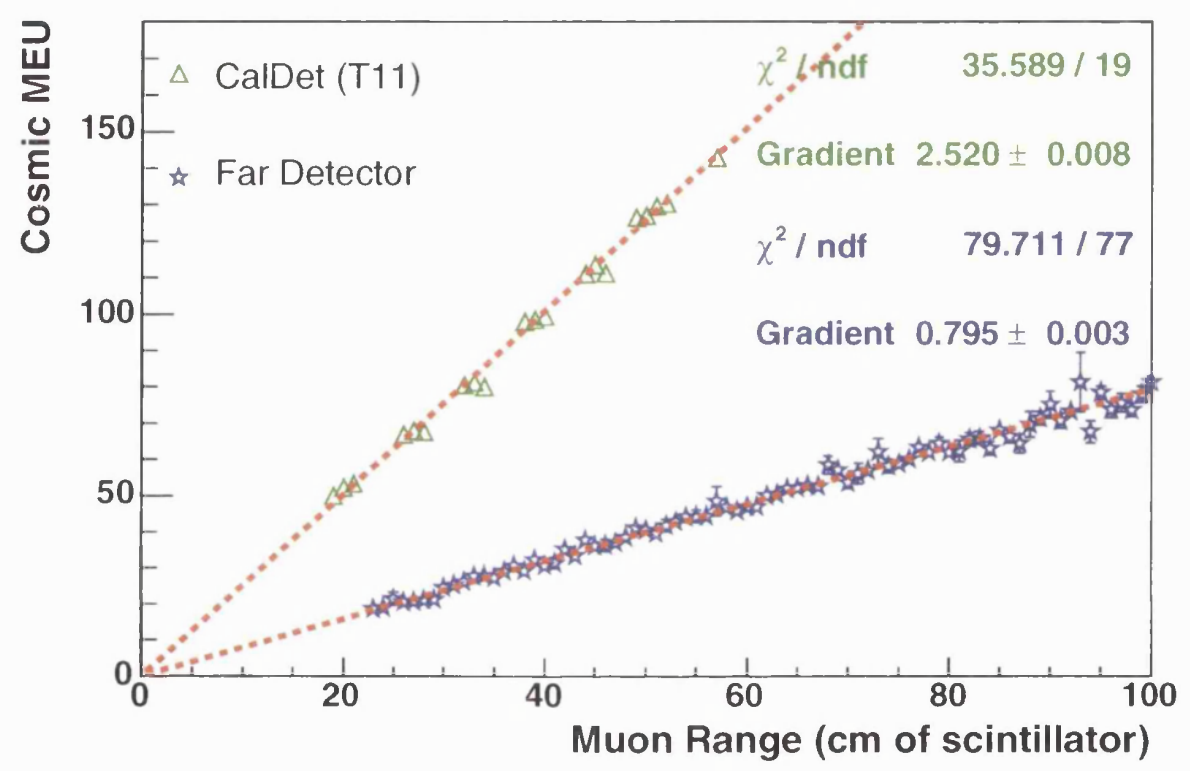

Figure 8.4: Demonstration of the viability of a cross-detector calibration using the total energy deposited by stopping muons in the Far and Calibration Detectors. The two detectors have different slopes due to the different cosmic ray muon spectra in the Soudan mine and at CERN. The average muon is much more energetic at the Far Detector, $\sim 100 \mathrm{GeV}$ compared to $\sim 4 \mathrm{GeV}$ at CalDet, this leads to a Far Detector cosmic MEU corresponding to a larger amount of energy than a CalDet cosmic MEU. Therefore the total energy against range slope is shallower at the Far Detector than at the CalDet, because of the different MEU scaling. The slopes of these two distributions can be used to calibrate the arbitrary cosmic MEU scales, allowing measurements from the CalDet to be applied to the Far Detector.

Using the slopes from the two detectors it is possible to cross-calibrate the arbitrary cosmic MEU scales used. These scales differ by more than a factor of three 
due to the different cosmic muon energy spectrum in each location. The cosmic muons in the Far Detector are much more energetic, due to its location half a mile underground. Taking the error on the slopes as an estimate of the statistical error on the calibration, the calibration is good to better than the $1 \%$ level.

\section{Caveat}

The only sample of stopping muons available in the Calibration Detector are beam muons. Due to the energy of cosmic ray muons at the Earths surface and the small transverse size of the CalDet, only $1 \mathrm{~m}$ across, nearly all of the cosmic rays pass through the detector. Those which do stop in the detector are hard to identify unambiguously as they stop close to the edge of the detector and look very similar to through going muons.

At the Far Detector there are no beam muons to use for the cross-detector calibration, therefore stopping cosmic ray muons must be used. The angular distribution of these cosmic muons has much larger variation than that of the beam muons used at the CalDet. This is illustrated in Figure 8.5, where the muon track $\frac{d z}{d s}$ is plotted for both Far Detector stopping cosmic ray muons and Calibration Detector stopping beam muons. There are very few cosmic ray muons which enter the detector perpendicular to the scintillator planes with $\frac{d z}{d s} \sim 1$.

The greater angular variety in the Far Detector stopping muon distribution results in many more muons which deposit energy in multiple strips per plane. As discussed in section 7.2.1, the total visible energy deposited by a muon will be different depending on the number of strips per plane it traverses. This difference will result in a systematic error in the cross-detector calibration, as illustrated in Figure 8.6. The total visible energy deposited by stopping cosmic ray muons in the Far Detector as a function of range is plotted for three regions of $\frac{d z}{d s}$. As $\frac{d z}{d s} \rightarrow 1$, and the track approaches the perpendicular to the scintillator planes, the total observed energy increases. At larger angles to the perpendicular the total observed energy decreases, as the muon tends to traverse more than one scintillator strip per plane. There is a $4 \%$ discrepancy between the slopes of the high $\frac{d z}{d s}$ sample and the 

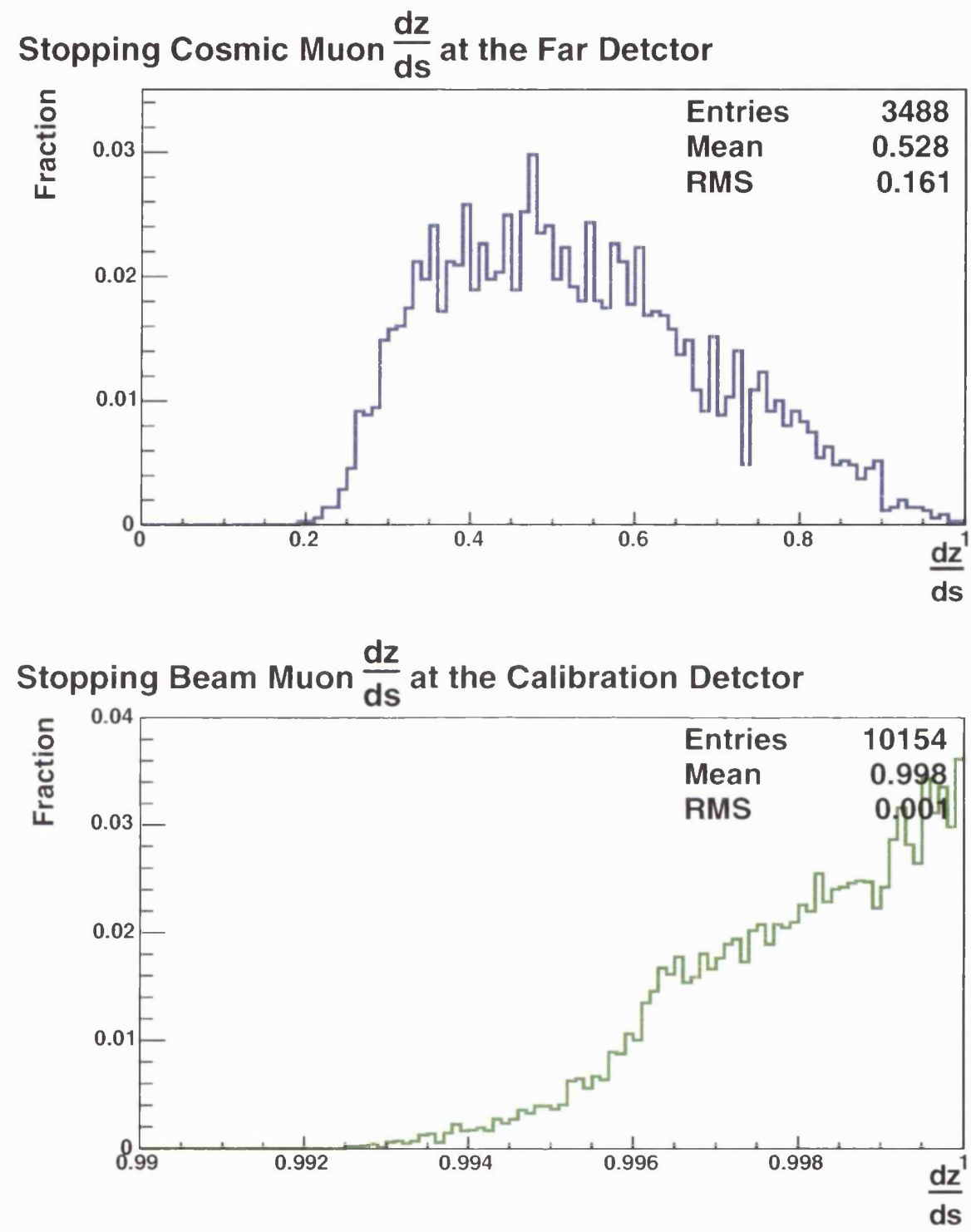

Figure 8.5: The $\frac{d z}{d s}$ of stopping muons both at the Far Detector and at the Calibration Detector. The top plot shows the distribution from stopping cosmic ray muons in the Far Detector, the bottom plot shows the distribution from stopping beam muons in the CalDet. Note the different scales of $\frac{d z}{d s}$ in the two plots. The beam muons at the CalDet enter the detector perpendicular to the scintillator planes, at values of $\frac{d z}{d s} \sim 1$. The cosmic ray muons in the Far Detector have a much larger angular variety, with very few muons at high values of $\frac{d z}{d s}$. 
total distribution, plotted in Figure 8.4. This discrepancy indicates that there is a systematic error on the slope plotted in Figure 8.4 of at least 4\%. This systematic uncertainty can be reduced by selecting out the most horizontal, those with $\frac{d z}{d s} \rightarrow 1$, muons from the sample. A sample with greater statistics is required to place tighter cuts than $\frac{d z}{d s}>0.7$, and to fully map out the dependence of the slope in Figure 8.4 on $\frac{d z}{d s}$, and thus minimize this systematic error.

\section{Total Energy Observed from Stopping Cosmic Ray Muons}

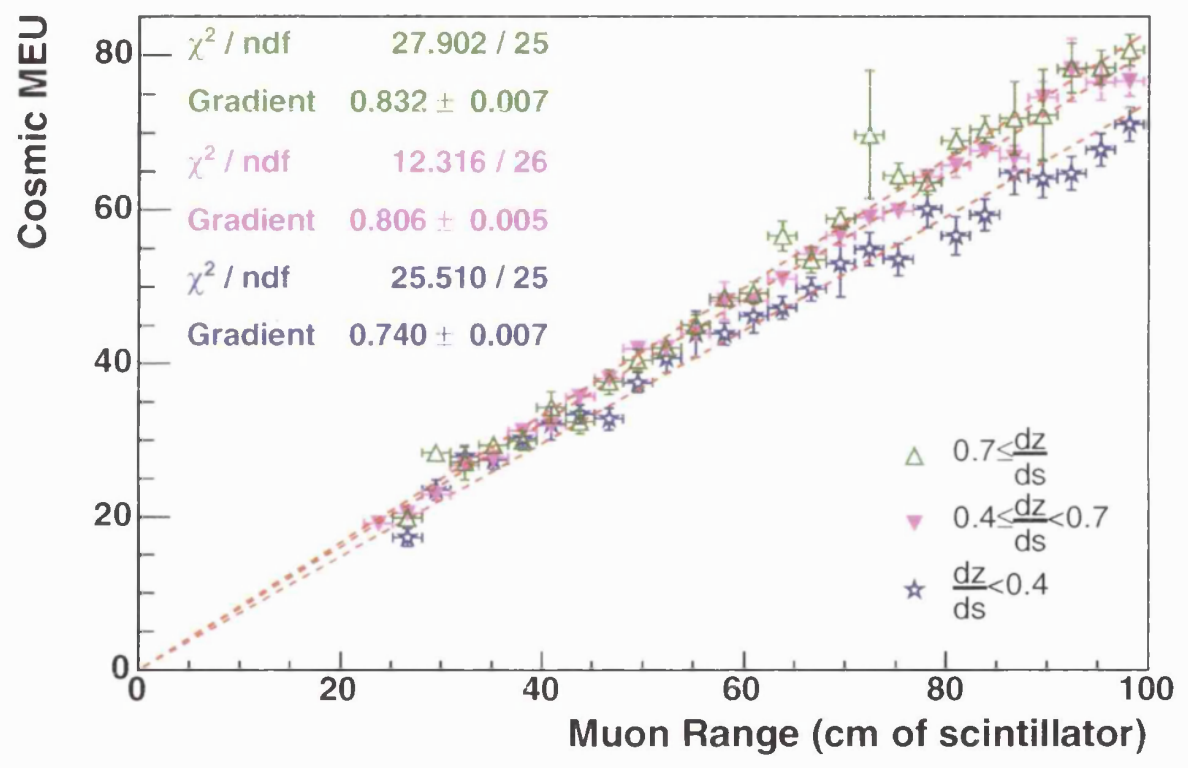

Figure 8.6: The total energy deposited by cosmic ray muons as a function of range, for three sections of the $\frac{d z}{d s}$ distribution. The total energy visible in the detector increases as the track approaches the perpendicular of the scintillator planes, i.e. as $\frac{d z}{d s} \rightarrow 1$. At larger angles to the perpendicular there are greater losses associated with muons depositing energy in more than one strip per plane. 


\subsection{Summary}

A preliminary cross-detector calibration has been performed between the Calibration and Far Detectors using stopping muons. In the Calibration Detector beam muons are used as there are very few stopping cosmic ray muons due to the cosmic energy spectrum and the small transverse size of the detector. At the Far Detector a sample of stopping cosmic ray muons from 35 days of livetime, accumulated over the course of two months in 2003, have been used. This calibration has a statistical error of less than $1 \%$, but due to the different muon angular spectrum used the calibration has a systematic error of at least $4 \%$.

A complete cross-detector calibration would require a sample of Far Detector muons travelling almost perpendicular to the scintillator planes, to minimize biases when compared to CalDet beam muons. However there are practically no cosmic ray muons that enter the detector close to horizontal due to the large quantity of rock between the detector and the ground level at such angles. Based on the statistics collected during 35 days of livetime, in one complete year of livetime, with both detectors operational, an estimated $730 \pm 120$ muons with $\frac{d z}{d s}>0.9$ will be observed. With these statistics it will be possible to perform a cross-detector calibration using nearly straight muons to within a $2 \%$ statistical error. The crossdetector calibration only needs to be performed once during the lifetime of the experiment, so many years of cosmic muons in the Far Detector may be used to perform the calibration. 


\section{Chapter 9}

\section{Measurement of the Charge}

\section{Distribution of Atmospheric}

\section{Neutrino induced Upward-Going}

\section{Muons}

An interesting feature of both the Near and Far Detectors is the fact that they are magnetized. The magnetic field allows some determination of the momentum and charge of muons whose momentum is below $100 \mathrm{GeV}$. The experiment will be the first neutrino experiment to have the capability of differentiating between $\nu_{\mu}$ and $\bar{\nu}_{\mu}$ events based upon the charge of the muons produced in the neutrino interactions. This capability will allow MINOS to make a number of interesting measurements, comparing the response and oscillation characteristics of neutrinos and anti-neutrinos. In the MINOS run plan there are periods of running with both a $\nu_{\mu}$ beam and also a $\bar{\nu}_{\mu}$ beam to systematically investigate their characteristics. The response of $\nu_{\mu}$ and $\bar{\nu}_{\mu}$ can also be investigated using atmospheric neutrinos; MINOS will be the first experiment to measure the charge distribution of these neutrinos.

In this chapter a muon track fitting algorithm, used to determine the momentum and charge of a muon from the track curvature in the magnetic field, will be described. An initial measurement of the charge distribution of upward-going 
muons induced from atmospheric neutrino interactions in the rock beneath the Far Detector will also be presented.

\subsection{Description of Track Fitter}

A muon track fitting algorithm, described in [73][55], used for the determination of muon momentum and charge from track curvature has been implemented for the MINOS detectors. The algorithm is based upon the least squares method used by CDHS.

Track fitting in the MINOS detectors is complicated by the large quantity of steel the muons pass through while traversing the detector. The energy loss in the iron leads to increased multiple scattering causing the muon path to deviate from the expected path due to curvature in the magnetic field. The toroidal nature of the magnetic field also complicates the momentum determination, as the field points in a different direction at every position along the track. Despite these complications it is relatively straightforward to obtain the muon momentum and charge, from curvature in the magnetic field, when the CDHS least squares method is used.

\subsubsection{The Least Squares Method}

The iterative least squares method is described fully in [73][55], only a brief description will be given here.

Positions along the reconstructed track are described by five fit parameters: the initial transverse position of the track $\left(u_{0}, v_{0}\right)$, the slope of the track at this point $\left(\left.\frac{d u}{d z}\right|_{0},\left.\frac{d v}{d z}\right|_{0}\right)$ and the reciprocal of the initial momentum $\frac{1}{p_{0}}$.

Initial values for these parameters are chosen based upon an extrapolation of the first few planes along the track, for the initial position and direction, and the range of the muon, for the initial momentum. Using these initial values the muon is 'swum' from plane to plane through the detector accounting for curvature in the magnetic field but not the effect of multiple scattering.

At each point on the reconstructed track the transverse positions $\left(u_{i}, v_{i}\right)$ are 
described by

$$
\begin{aligned}
& u_{i}=u_{0}+\left.\left(z_{i}-z_{0}\right) \frac{d u}{d z}\right|_{0}+\frac{1}{p_{0}} b_{i}^{u} \\
& v_{i}=v_{0}+\left.\left(z_{i}-z_{0}\right) \frac{d v}{d z}\right|_{0}+\frac{1}{p_{0}} b_{i}^{v},
\end{aligned}
$$

where $z_{i}$ is the current longitudinal position and by rearrangement $b_{i}^{u}$ and $b_{i}^{v}$ are given by

$$
\begin{aligned}
& b_{i}^{u}=\left(u_{i}-u_{0}-\left.\left(z_{i}-z_{0}\right) \frac{d u}{d z}\right|_{0}\right) p_{0} \\
& b_{i}^{v}=\left(v_{i}-v_{0}-\left.\left(z_{i}-z_{0}\right) \frac{d v}{d z}\right|_{0}\right) p_{0} .
\end{aligned}
$$

The expression for $\chi^{2}$ is

$$
\chi^{2}=\sum_{i, j}\left(u_{i}-u_{i}^{m}\right) \omega_{i j}^{u}\left(u_{j}-u_{j}^{m}\right)+\sum_{k, l}\left(v_{k}-u_{k}^{m}\right) \omega_{k l}^{v}\left(v_{k}-u_{l}^{m}\right)
$$

where $u_{i}^{m}$ and $v_{i}^{m}$ are the measured positions of the track, as measured by the positions of the strips in the scintillator planes, and $\omega_{i j}^{X}$ are the elements of weight matrices, defined separately for each of the two strip orientations, like

$$
\left(\begin{array}{cccc}
\epsilon_{1}^{2}+\left\langle P_{1}^{2}\right\rangle & \left\langle P_{1} P_{2}\right\rangle & \left\langle P_{1} P_{3}\right\rangle & \ldots \\
\left\langle P_{1} P_{2}\right\rangle & \epsilon_{2}^{2}+\left\langle P_{2}^{2}\right\rangle & \left\langle P_{2} P_{3}\right\rangle & \ldots \\
\left\langle P_{1} P_{3}\right\rangle & \left\langle P_{2} P_{3}\right\rangle & \epsilon_{3}^{2}+\left\langle P_{3}^{2}\right\rangle & \ldots \\
\ldots & \ldots & \ldots & \epsilon_{N}^{2}+\left\langle P_{N}^{2}\right\rangle
\end{array}\right)^{-1}
$$

where $\epsilon_{i}$ is the measurement error on the transverse position at plane $i$ and $\left\langle P_{n} P_{m}\right\rangle$ are the covariances between the two planes, these will be defined later.

The minimum $\chi^{2}$ is found by setting the five partial derivatives $\frac{\partial \chi^{2}}{\partial u_{0}}, \frac{\partial \chi^{2}}{\partial v_{0}}, \frac{\partial \chi^{2}}{\left.\partial \frac{d u}{d z}\right|_{0}}$, $\frac{\partial \chi^{2}}{\left.\partial \frac{d v}{d z}\right|_{0}} \& \frac{\partial \chi^{2}}{\partial \frac{1}{p_{0}}}$ to zero, this leads to five equations with five unknowns. These equations can be expressed in matrix form as $\mathbf{A B}=\mathbf{C}$, with $\mathbf{A}$ given by

$$
\left(\begin{array}{ccccc}
\sum 2 \omega_{i j} & \sum \omega_{i j}\left(z_{i}+z_{j}\right) & \sum \omega_{i j}\left(b_{i}^{u}+b_{j}^{u}\right) & 0 & 0 \\
\sum \omega_{i j}\left(z_{i}+z_{j}\right) & \sum 2 \omega_{i j}\left(z_{i} z_{j}\right) & \sum \omega_{i j}\left(b_{i}^{u} z_{j}+b_{j}^{u} z_{i}\right) & 0 & 0 \\
\sum \omega_{i j}\left(b_{i}^{u}+b_{j}^{u}\right) & \sum \omega_{i j}\left(b_{j}^{u} z_{i}+b_{i}^{u} z_{j}\right) & \sum 2 \omega_{i j}\left(b_{i}^{u} b_{j}^{u}\right)+\sum 2 \omega_{k l}\left(b_{k}^{u} b_{l}^{v}\right) & \sum \omega_{k l}\left(b_{k}^{v}+b_{l}^{u}\right) & \sum \omega_{k l}\left(b_{k}^{u} z_{l}+b_{l}^{v} z_{k}\right) \\
0 & 0 & \sum \omega_{k l}\left(b_{k}^{u}+b_{l}^{u}\right) & \sum \sum \omega_{k l} & \sum \omega_{k l}\left(z_{k}+z_{l}\right) \\
0 & 0 & \sum \omega_{k l}\left(b_{l}^{u} z_{k}+b_{k}^{v} z_{l}\right) & \sum \omega_{k l}\left(z_{k}+z_{l}\right) & \sum 2 \omega_{k l}\left(z_{k} z_{l}\right)
\end{array}\right)
$$


and

$$
\mathbf{B}=\left(\begin{array}{c}
u_{0} \\
\left.\frac{d u}{d z}\right|_{0} \\
\frac{1}{p_{0}} \\
v_{0} \\
\left.\frac{d v}{d z}\right|_{0}
\end{array}\right), \quad \mathbf{C}=\left(\begin{array}{c}
\sum \omega_{i j}\left(u_{i}^{m}+u_{j}^{m}\right) \\
\sum \omega_{i j}\left(u_{i}^{m} z_{j}+u_{j}^{m} z_{i}\right) \\
\sum \omega_{i j}\left(u_{i}^{m} b_{j}^{u}+u_{j}^{m} b_{i}^{u}\right)+\sum \omega_{k l}\left(v_{k}^{m} b_{l}^{v}+v_{l}^{m} b_{k}^{v}\right) \\
\sum \omega_{k l}\left(v_{k}^{m}+v_{l}^{m}\right) \\
\sum \omega_{k l}\left(v_{k}^{m} z_{l}+v_{l}^{m} z_{k}\right)
\end{array}\right)
$$

The matrix $\mathbf{A}$ is inverted leading to solutions for the five parameters, from $\mathbf{B}=\mathbf{A}^{-1} \mathbf{C}$, these values are used in the next iteration. The iterative process continues until a convergence criteria is satisfied; the criteria used is that the successive values of the momentum differ by less than $200 \mathrm{MeV}$. The $200 \mathrm{MeV}$ value is an empirical number based upon the performance of the algorithm, although it is worth noting that in the spectrometer section of the Near Detector the energy lost between scintillator planes is approximately $200 \mathrm{MeV}$.

\subsubsection{Treatment of Errors}

For an accurate determination of the fit parameters and their uncertainties, all major sources of error must be included in the calculation of the weight matrix elements.

The largest source of errors for muons in the MINOS detectors is due to multiple scattering. As the muon passes through each steel-air-scintillator layer of the detector it undergoes multiple scattering. The errors at each scintillator plane are correlated because small scatters in each layer must be projected forwards to the end of the muon track, thus causing the total deviation in the final plane to be the sum of all previous scatters.

Another factor which must be considered is the energy dependence of the mean square scattering angle, $\theta_{0 i}^{2}$, which depends on the energy of the muon as it traverses layer $i$. The variation in displacement due to multiple scattering and energy loss at plane $N$ and the covariance between the displacements at planes $K$ and $N$ are 
given by

$$
\left\langle P_{N}^{2}\right\rangle=\sum_{i=1}^{N} \theta_{0 i}^{2}\left(\frac{x_{i}^{2}}{3}+\mathcal{D}(i, N)\left(x_{i}+\mathcal{D}(i, N)\right)\right)
$$

and

$$
\left\langle P_{K} P_{N}\right\rangle=\sum_{i=1}^{K} \theta_{0 i}^{2}\left(\frac{x_{i}^{2}}{3}+\frac{x_{i}}{2}(\mathcal{D}(i, K)+\mathcal{D}(i, N))+\mathcal{D}(i, K) \mathcal{D}(i, N)\right),
$$

where $x_{i}$ is the distance travelled by the muon in layer $i, \mathcal{D}(i, L)$ is the total distance along the path of the muon from layer $i$ to $L$ and $\theta_{0 i}^{2}$ is given by

$$
\theta_{0 i}^{2}=\frac{0.0136}{p_{i} \beta c} \sqrt{\frac{x_{i}}{X_{0}}}\left[1+0.038 \ln \left(\frac{x}{X_{0}}\right)\right] .
$$

with $p_{i}$ the muon momentum in layer $i$ and $X_{0}$ the radiation length of the steelair-scintillator layer. These covariances must be used if a realistic error on the fit parameter is to be obtained.

\subsubsection{Performance of Track Fitter}

The track fitting algorithm described has been tested using both Monte Carlo data and data from the Far Detector.

\section{Monte Carlo Results}

A sample of cosmic ray Monte Carlo events have been generated using the GMINOS, GEANT3 based, simulation package. The full MINOS reconstruction chain [74], using the least squares track fitting algorithm described above, has been applied to this data set.

The purpose of track fitting is to determine the momentum and charge of a muon from the track curvature in the magnetic field. Figure 9.1 shows the muon momentum spectrum, both from truth information and as determined from the fit. In the figure negative momenta are from $\mu^{-}$and positive momenta from $\mu^{+}$. The truth spectrum is shown both for all events and also for only those events which are successfully fitted, approximately $66 \%$ of the total. The vast majority of unfitted tracks are short tracks of less than 16 planes, where there is little information 
available for the track fitter. Even very high energy muons can leave short tracks in the detector, depending on their position and direction when they enter the detector. All three distributions have the same coarse features, although the fitted momentum distribution is more peaked than the truth distributions.

The importance of track length is illustrated in Figure 9.2, the ratio $\frac{\frac{1}{p_{\text {fit }}}-\frac{1}{p_{\text {true }}}}{\frac{1}{p_{\text {true }}}}$ is plotted as a function of number of planes traversed by the muon. As one would expect if the momentum determination is accurate, the distribution is centred around zero and as the number of planes increases the distribution becomes tighter. This is because as the number of planes traversed increases the amount of information available to the fit also increases.

Another factor which influences the ability of the track fitting algorithm to accurately determine the charge and momentum of a muon is the momentum itself. The classical equation governing the motion of a particle of momentum $p$ and charge $q$ in a constant magnetic field $B$ is

$$
r=\frac{p}{q B}
$$

where $r$ is the radius of curvature of the particle. The higher the particle's momentum the less it curves through the magnetic field. The same is obviously true for muons bending in the toroidal magnetic field of the Far Detector. High momentum muons do not have their trajectories altered significantly by the magnetic field as they pass through the detector. Therefore both the charge identification and momentum determination capabilities decrease at high momenta. This is illustrated in Figure 9.3, the ratio $\frac{p_{\text {fit }}}{p_{\text {true }}}$ is plotted for all fitted muons and also separately for those muons with momentum measured to be less than $50 \mathrm{GeV}$. Some $15.6 \%$ of the events have their charge misidentified, when only those events whose fitted momentum is less than $50 \mathrm{GeV}$ are considered this misidentification is reduced to only $3.3 \%$. There is a limiting factor on the ability to correctly identify charge due to the multiple scattering as the muons traverse the detector. These scatters can cause the muon to leave a straighter track than would be expected from curvature, or even to kink in the opposite direction to the curvature. In these cases an accurate determination of the charge and momentum of the muon is not possible. 


\section{Momentum Spectrum From Truth}

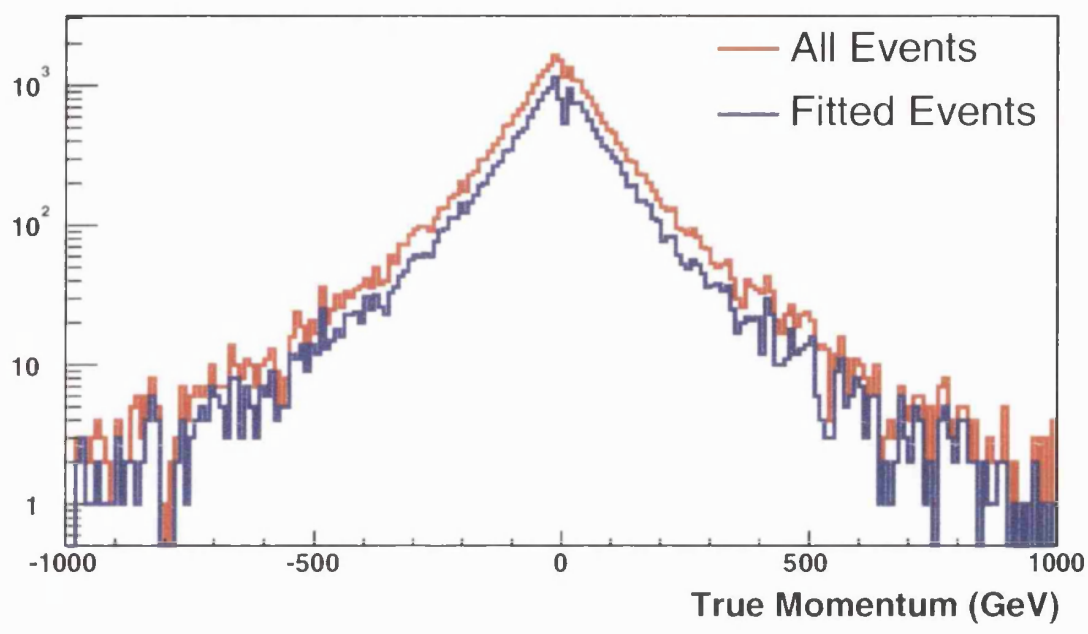

\section{Momentum Spectrum From Fit}

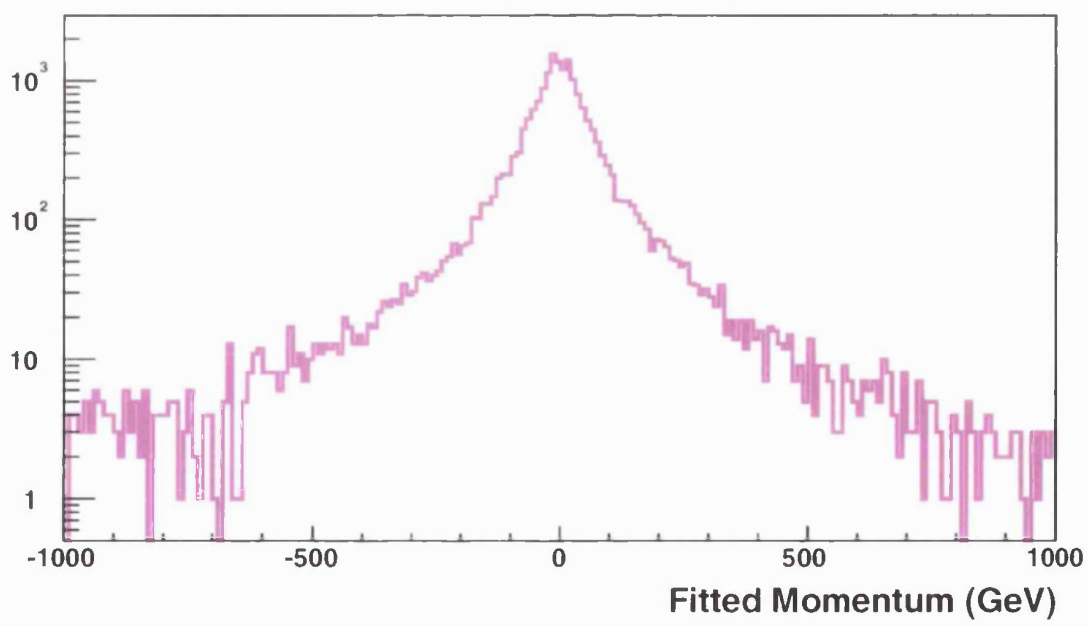

Figure 9.1: The moment um spectrum from a sample of simulated Far Detector cosmic ray muons. Negative values of muon momentum corresponds to $\mu^{-}$and positive values to $\mu^{+}$. The top two plots show the actual momentum of the generated particles (the "truth"), for both all muon events and the $66 \%$ of events for which the least squares fit converges. The bottom plot shows the momentum as determined by the least squares fit. 


\section{Fractional Deviation from True Muon Momentum}

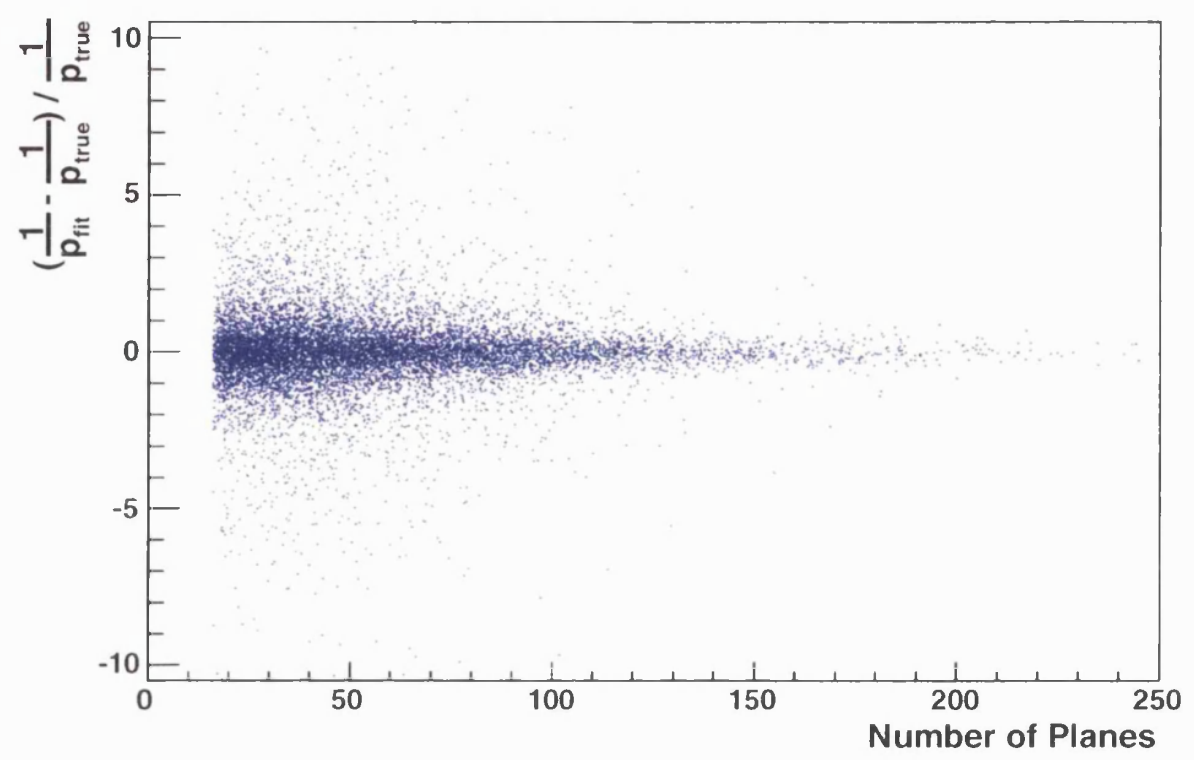

Figure 9.2: The momentum determination of the least squares fitter as a function of the number of planes traversed by the muon. The ratio $\frac{\frac{1}{p_{f i t}}-\frac{1}{p_{\text {true }}}}{\frac{1}{p_{\text {true }}}}$ should be a tight distribution centred around zero if the momentum is well determined. As the number of planes traversed increases the momentum is better determined, and the number of outlying events is decreased.

The efficiency of charge identification as a function of true muon energy is shown in Figure 9.4. Below $50 \mathrm{GeV}$ over $90 \%$ of muons have their charge correctly determined. For energies above $\sim 100 \mathrm{GeV}$ the efficiency declines rapidly and all that can be determined from the fit is that the muon was highly energetic, with essentially no information about the charge of the muon.

Studies have been conducted into the performance of the fitting algorithm as a function of particle energy [55], these studies show that for fully contained tracks the momentum resolution varies from $\sim 30 \%$ for $4 \mathrm{GeV}$ muons to $12 \%$ for $15 \mathrm{GeV}$ muons. For these fully contained tracks the momentum is measured with a resolution of approximately $6 \%$ using the track length. The major limiting factor on the resolution from curvature is multiple scattering, this uncertainty grows almost 


\section{Momentum from Fit / True Momentum}

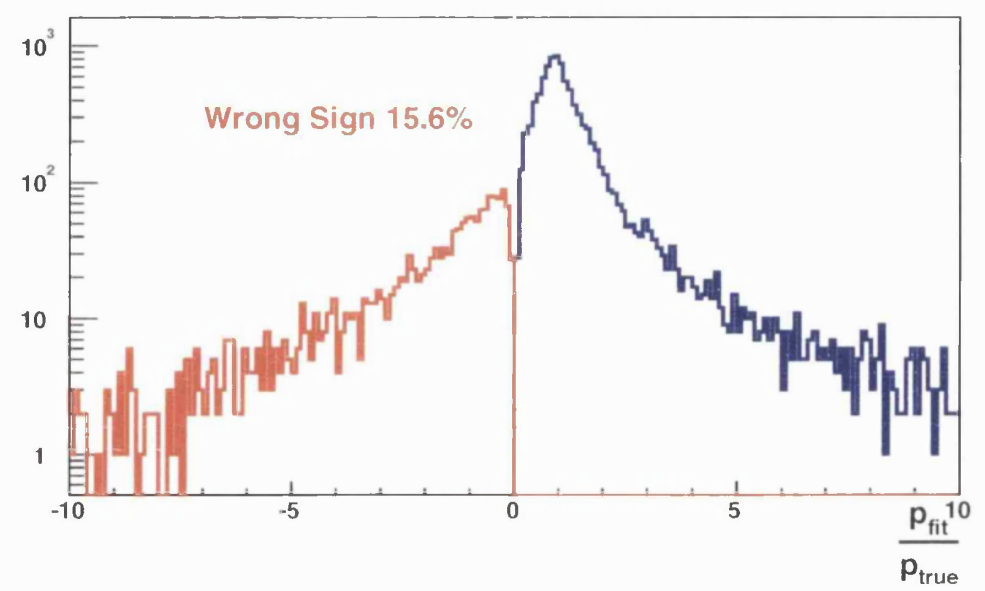

Momentum from Fit / True Momentum (Sub $50 \mathrm{GeV}$ )

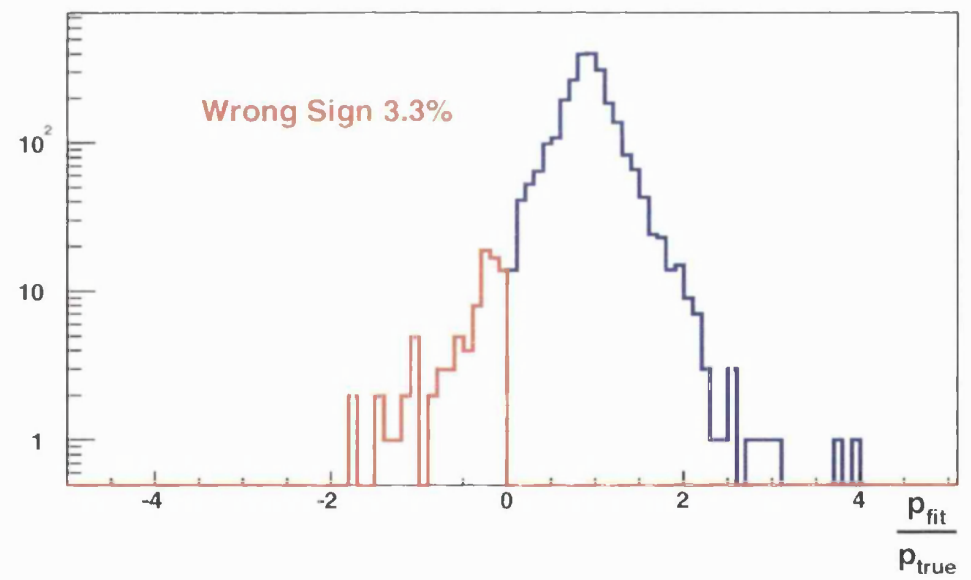

Figure 9.3: The ratio $\frac{p_{\text {fit }}}{p_{\text {true }}}$ is plotted in the top plot for all fitted events and in the bottom plot just for those events whose momentum is measured to be below $50 \mathrm{GeV}$. The ratio is peaked at 1 , the value where the momentum and charge determined by the fitting algorithm are accurate. The long tails in the top distribution are due to high energy muons which do not curve significantly in the magnetic field, so their momentum is not well measured. The $15.6 \%$ charge misidentification of all events is reduced to $3.3 \%$ when only those events with momentum below $50 \mathrm{GeV}$ are considered. 


\section{Efficiency of Charge Identification}

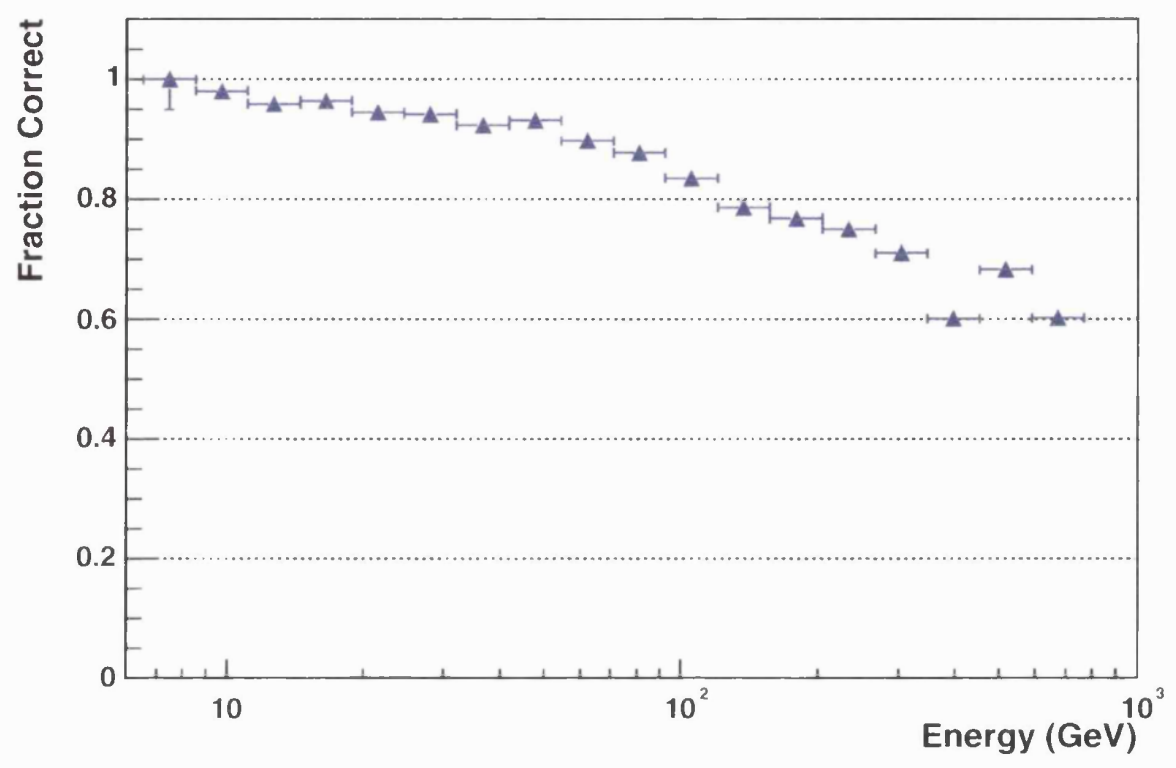

Figure 9.4: The efficiency of muon charge identification, from curvature in the magnetic field, is plotted as a function of muon energy. The efficiency decreases with energy as more energetic particles curve less in the magnetic field. This results in an inability to determine the direction of the curvature and hence the charge of the muon is not well determined. Below $50 \mathrm{GeV}$ the charge is determined correctly for over $90 \%$ of the events.

exponentially with track length. 


\section{Results from Far Detector Cosmic Ray Data}

The track fitting algorithm has also been tested using a sample of cosmic ray muons from the Far Detector. The momentum spectrum from these events is shown in Figure 9.5, as before negative momenta correspond to $\mu^{-}$and positive momenta to $\mu^{+}$. Qualitatively there is a good agreement between this distribution and that from the simulation shown in Figure 9.1.

\section{Momentum Spectrum (from Fit to Data)}

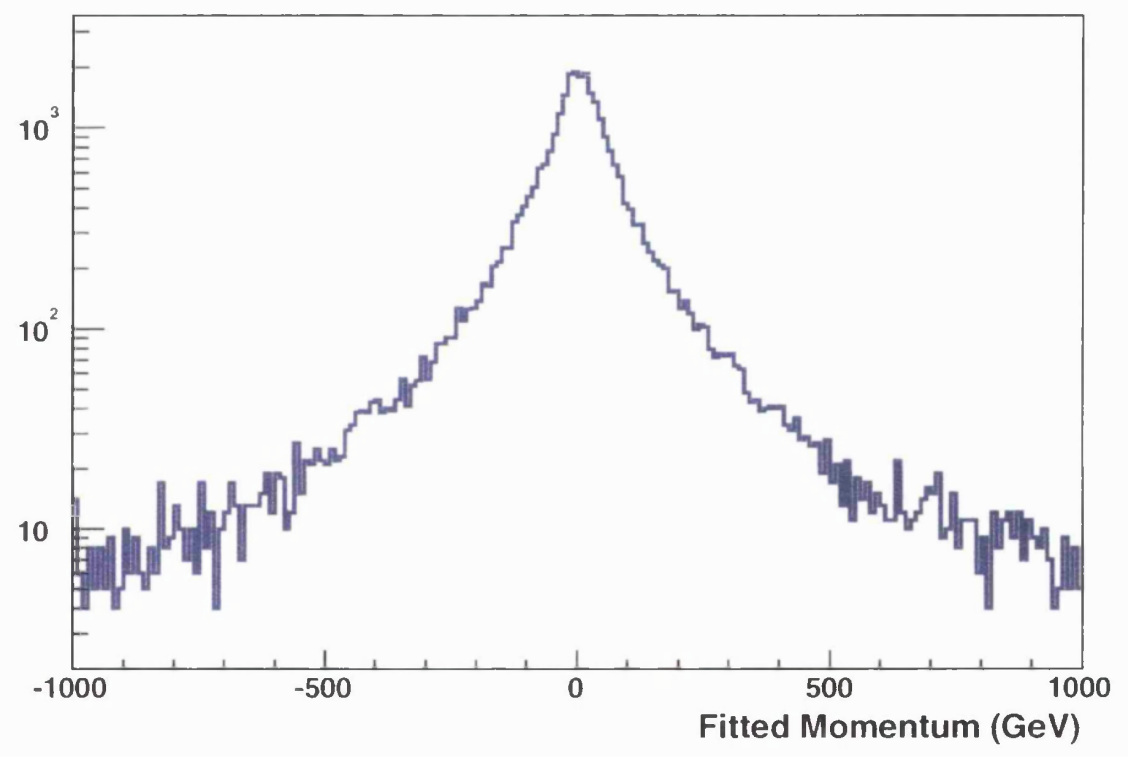

Figure 9.5: The momentum spectrum of cosmic ray muons in the Far Detector, measured from track curvature in the magnetic field. The distribution is in good agreement with that shown in Figure 9.1 from simulated data.

One of the main features of the track fitting algorithm is the realistic treatment of errors due to measurement error, energy loss and multiple scattering. The fractional error on muon momentum obtained from the method is plotted as a function of the number of planes traversed by the muon in Figure 9.6. As is expected from comparison with the deviation from truth distribution plotted in Figure 9.2, the fractional error decreases as the track length increases and more information is available. The fractional error distribution is the tighter of the two, due in part to 
the tendency for momentum to be overestimated in the case of those tracks which are almost straight. Above $100 \mathrm{GeV}$ there is very little information which can be gained from track curvature and realistically only minimum energy bounds can be set.

\section{Fractional Error on Muon Momentum}

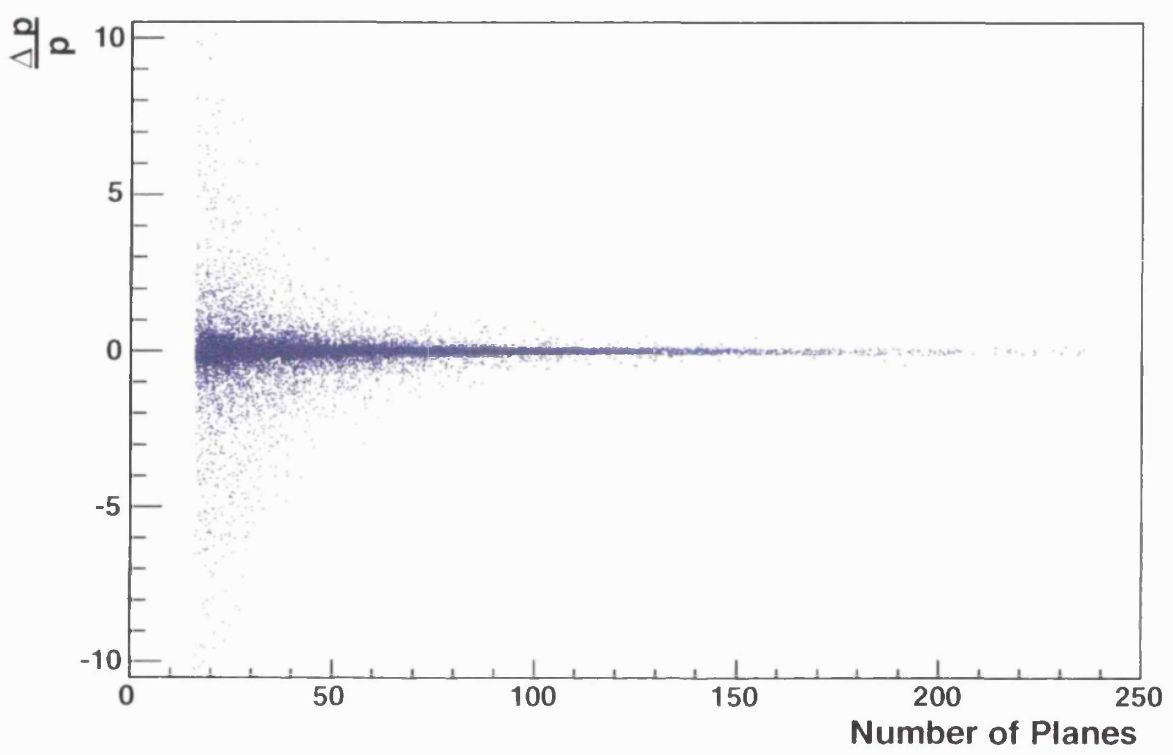

Figure 9.6: The fractional error on muon moment um is plotted as a function of the number of planes in the muon track. As expected the fractional error decreases with increasing track length, as the track fitter has more information available from the longer tracks. 


\subsection{Upward-going Muons at the Far Detector}

The Far Detector is located at the bottom of the Soudan mine and as such it is in the perfect location to study atmospheric neutrinos. There are two varieties of atmospheric neutrino related events visible in the detector: neutrinos which interact in the detector itself and those $\nu_{\mu}$, or $\bar{\nu}_{\mu}$ which interact in the rock surrounding the detector producing muons which enter the detector. It is this second class of neutrinos that we are concerned with in this section.

Neutrinos can travel a long distance through the Earth before interacting, as the cross-section for neutrino interactions is very small as is shown in Figure 9.7. The average value of $\sigma_{T} / E_{\nu}$ for $\nu_{\mu}$ is $0.677 \pm 0.014 \times 10^{-38} \mathrm{~cm}^{2} / \mathrm{GeV}$ and for $\bar{\nu}_{\mu}$ it is $0.334 \pm 0.008 \times 10^{-38} \mathrm{~cm}^{2} / \mathrm{GeV}$. In fact, the cross-section is so small that the vast majority of neutrinos pass through the Earth without interacting. The small percentage of $\nu_{\mu}$ and $\bar{\nu}_{\mu}$ which do interact produce muons which, depending on the energy of the neutrino, can travel for up to $1 \mathrm{~km}$ in the rock. These factors combined with the flux of atmospheric neutrinos (neutrinos produced from cosmic ray interactions in the atmosphere) mean that atmospheric neutrino induced muons can enter the detector from any direction.

Those muons which enter the detector from above can not be distinguished from the background of cosmic ray muons. Only those muons which enter the detector either horizontally or travelling upwards can be identified as neutrino induced muons. The flux of cosmic ray muons from these angles is essentially zero due to the large quantity of rock between the detector and the surface of the Earth in those directions. The only source of background comes from cosmic ray muons which experience a hard scatter in the rock near the detector causing a drastic change in the direction of the muon, such that it enters the detector travelling horizontally or upwards. From simulations of cosmic ray muons carried out for the Soudan II experiment using the rock overburden in the Soudan mine, this background is calculated to be at least two orders of magnitude below the expected flux from atmospheric neutrinos [75].

The charge distribution of these upward-going muons is directly linked to the 


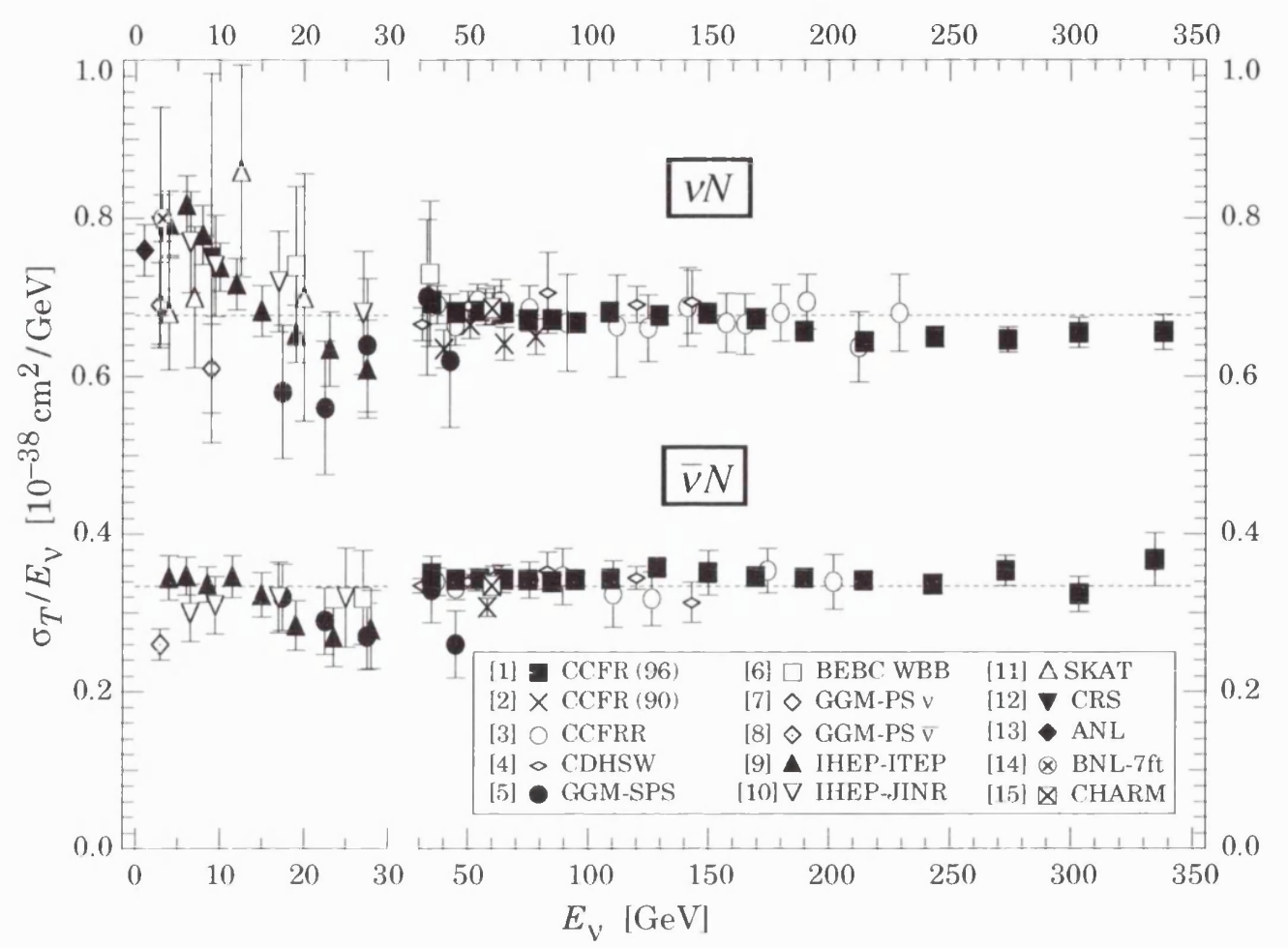

Figure 9.7: Summary of measurements made of the charged current $\nu_{\mu}$ and $\bar{\nu}_{\mu}$ crosssections. The symbols used in this plot are explained in [51]. The average value of $\sigma_{T} / E_{\nu}$ for $\nu_{\mu}$ is $0.677 \pm 0.014 \times 10^{-38} \mathrm{~cm}^{2} / \mathrm{GeV}$ and for $\bar{\nu}_{\mu}$ it is $0.334 \pm 0.008 \times 10^{-38} \mathrm{~cm}^{2} / \mathrm{GeV}$. From [51].

neutrino/anti-neutrino content of the atmospheric neutrinos, as the muons are produced through the following interactions:

$$
\begin{gathered}
\nu_{\mu}+e^{-} \rightarrow \mu^{-}+\nu_{e} \\
\nu_{\mu}+d \rightarrow \mu^{-}+u \\
\bar{\nu}_{\mu}+u \rightarrow \mu^{+}+d .
\end{gathered}
$$

The quark interactions are favoured by cross-section.

An event sample of over 3.3 million events has been used in the following analysis. This event sample was obtained between October 2002 and June 2003 from over 102 days of detector operation, or approximately two thirds of a kiloton year. The expected number of upward-going muons in the absence of neutrino oscillations based upon scaling a simulation performed by $B$. Rebel [76] is 12 events. The 
simulation predicts some 400 events in 5 years with both super-modules active, with the ratio of $\mu^{-}: \mu^{+}$events to be $280: 120$.

\subsubsection{Selection of Upward-Going Muons}

The major problem with selecting a sample of upward-going muons is in removing those cosmic ray muons whose directionality is ambiguous due to event topology and timing information. A number of cuts are applied to the event sample to ensure that a pure sample of upward-going muons is selected with high efficiency. The full MINOS reconstruction software, using the least squares track fitting method described, has been applied to this data set. The cuts applied to the data to select a sample of events with good timing information are based in part upon the analyses shown in [76][77], they are:

i) The event must contain a single track with no fewer than 20 planes and be over $2 \mathrm{~m}$ long. As has been shown above the charge and momentum determination improves as the track length increases. Also each PMT in the detector is timestamped independently, recall that three PMTs readout one end of a pair of planes, so as the number of planes traversed increases there are more time measurements and the timing resolution improves. The downside of a minimum track length cut is that it imposes a energy threshold on the muons. From comparison with CalDet data a track length of $2 \mathrm{~m}$ corresponds to muons of momentum $1.2 \mathrm{GeV}$.

ii) The vertex of the event must lie within $0.3 \mathrm{~m}$ of one of the edges of the detector. This is to remove any events which are misreconstructed with a vertex outside the physical volume of the detector, and also the events from neutrinos interacting in the detector itself.

iii) The number of strips identified as being part of a shower must be less than the number of strips along the track. Some muons produce large showers as they traverse the detector, these can confuse the track fitting.

iv) The event must not be tagged as a multiple muon event, as these can confuse the both the event reconstruction, in particular the demuxing. Recall that each 
PMT pixel in the Far Detector is connected to 8 scintillator strips, and using event topology and the double ended readout the correct strip for each pixel must be selected.

v) The track must not enter the unmagnetized (at the time of data collection) second super-module (SM2). This ensures that there is curvature information for the full length of the track.

vi) The transverse position along the strip as measured by strip position in the other orientation must be consistent with the transverse position obtained using the timing of hits at either end of the scintillator strip. For strips which are read out at both ends it is possible to estimate the transverse position along the strip at which the muon crossed. This provides a useful crosscheck for the reconstruction, to ensure the correct demuxing solution was selected.

vii) The time gradient with respect to vertical, $y$, position must agree with the direction cosine in the vertical direction at the vertex of the event. Ensuring that both event topology and timing information suggest the particle is travelling in the same direction. In the MINOS Far Detector electronics the digits are timestamped on a PMT basis, with three PMTs reading out one end of a pair of planes. Only the first hit strip (along the path of the track) is used in the straight line fit determining the time slope.

viii) The reduced $\chi^{2}$ from the straight line fit to the time against $y$ position distribution must be less than 5 . This is to remove events which have ambiguous timing.

ix) The magnitude of the time gradient must be greater than the error on it as reported by the straight line fit.

x) The time gradient must be positive for the upward going muon sample.

xi) For those events which have a positive time gradient the final stage in event identification is a visual scanning to confirm the events look as though they are consistent with being upward-going muons. This is to remove the few remaining misreconstructed events which do not look physical but are hard to remove using topological and timing cuts. This scanning is only possible due to the small number of events which pass the above cuts. 
Some of these cuts are necessary to ensure a clean sample of muons, with no obvious reconstruction problems or contamination from showering particles. Unsurprisingly, the most critical cuts for selecting a sample of upwards going muons are those which are based upon the timing distribution of the hits along the track. Figure 9.8 shows the distribution of time gradients from straight line fits to the time as a function of $y$ position. The distribution of time gradients is plotted after cuts viii) and $\mathrm{ix}$ ) are applied, the candidate upward-going muon events have positive values of this gradient. Also shown in Figure 9.8 is the distribution of time gradients close to 0 , illustrating the effect of the two timing cuts.

\section{Time - Y Position Gradient}

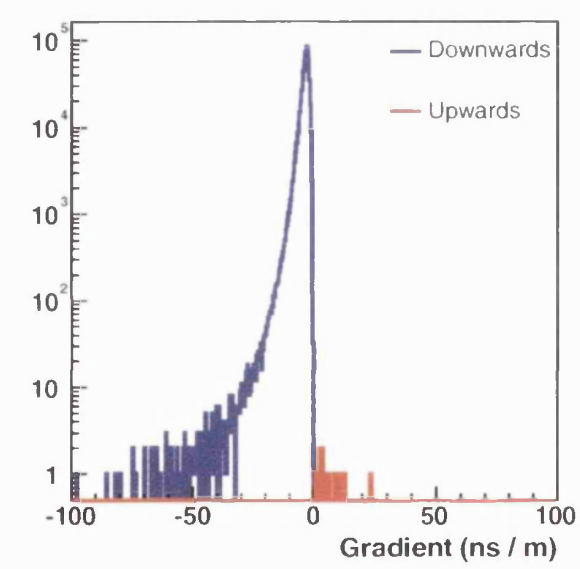

\section{Effect of Timing Cuts}

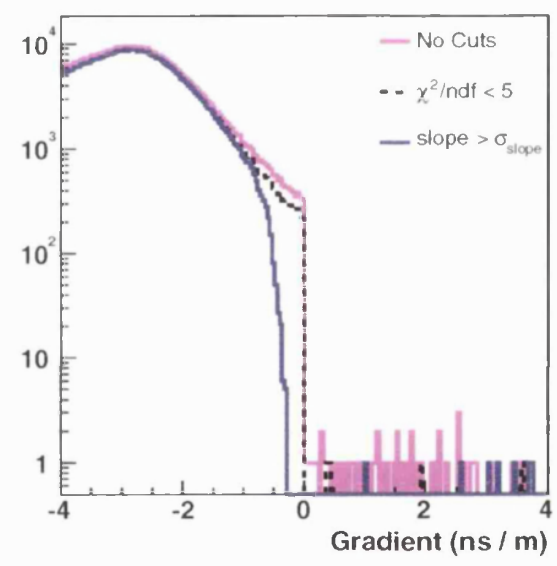

Figure 9.8: The lefthand plot shows the distribution of time- $y$ position gradients from straight line fits after cuts on the quality of the fit are applied. The candidate upward going muon events are shown with positive gradients. The righthand plot shows how the timing cuts, $\chi^{2} / n d f<5$ and $\mid$ slope $\mid>\sigma_{\text {slope }}$ effect the timing distribution. These cuts are intended to remove events with ambiguous timing information.

Another way of visualising the timing quality cuts is to see the effect of the cuts on the vertical angular distribution of the muons, this is plotted in Figure 9.9. The timing cuts remove a large fraction of the candidate upward-going events, most likely these are events in which the muon is reconstructed travelling in the opposite direction to which it actually was travelling. 


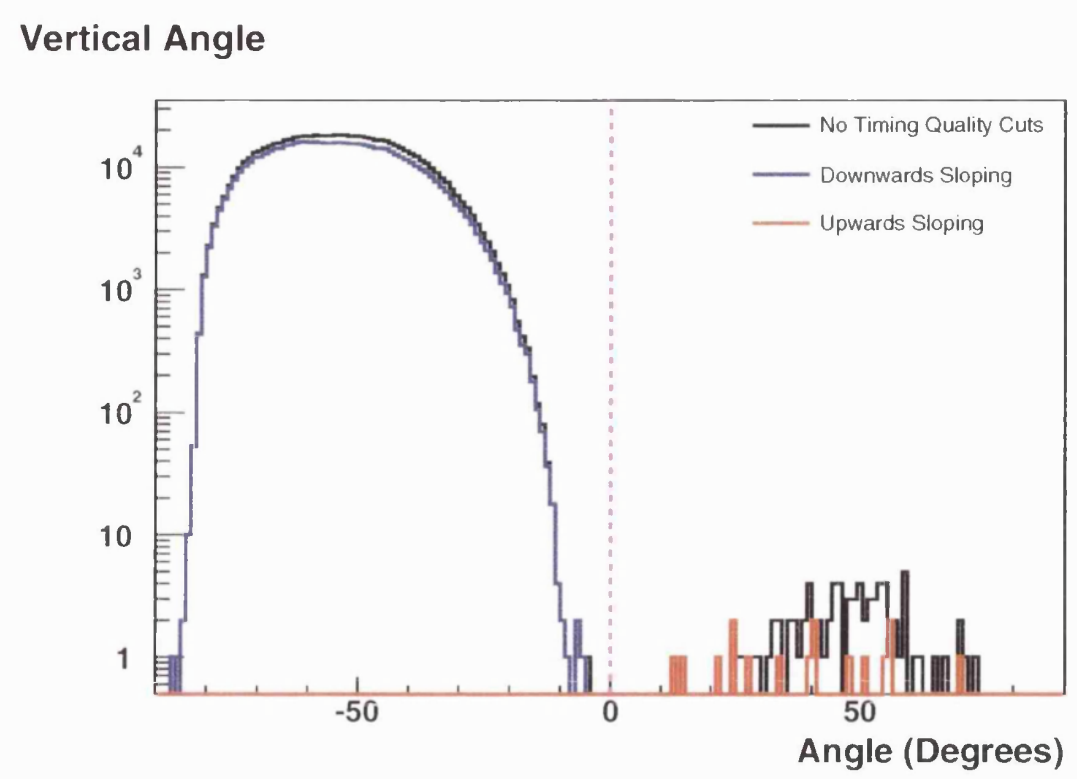

Figure 9.9: The angular distribution of muons with respect to the horizontal plane. Angles greater than zero correspond to muons reconstructed as travelling upwards and negative angles to muons travelling downwards. The distribution is plotted in black for all those events for which the track is reconstructed with a vertical angle in agreement with the time gradient, before quality cuts are applied to the timing. After the timing quality cuts are applied and those events with ambiguous timing are removed, the number of candidate upward-going muon events is reduced, leaving a cleaner sample of upward-going muons.

The effect of the individual cuts is illustrated in Figure 9.10. The cuts are applied from left to right. Those requiring a long track in the first super module remove the largest number of events. Followed by the cuts requiring a physical solution with a good fit to the time distribution. 


\section{Efficiency of Cuts}

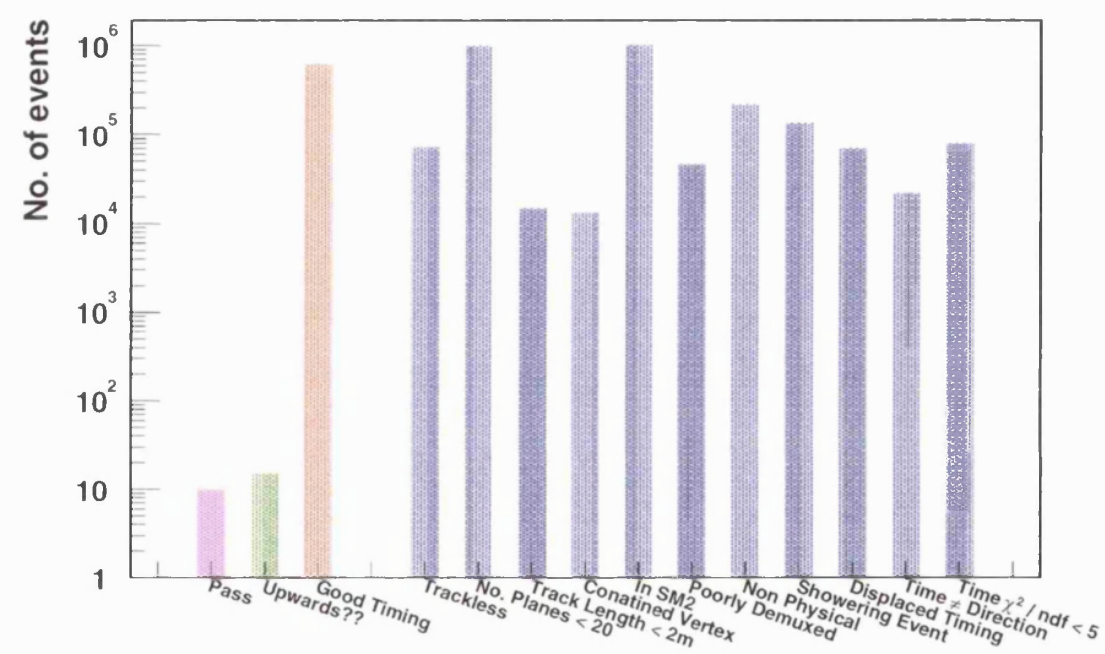

Figure 9.10: The relative efficiency of the cuts used to select a sample of upward-going muons. The cuts, shown in blue, are applied on the sample from left to right. From the initial sample of 3308805 events only $618143(21 \%)$ are events in super module one with good timing information. Of these a mere 10 events are upward-going muons with no obvious misreconstruction. 


\subsubsection{Results}

After all the cuts are applied we are left with a sample of 10 upward-going muons, one of which is illustrated in Figure 9.11. As with the Calibration Detector crosstalk in the M16 PMTs is a cause of confusion for the tracking algorithm. The majority of the hits below $2 \mathrm{PE}$ are probably the result of crosstalk, although some of these are due to energy deposits by particles (primarily electrons and photons) related to the muon. This is a particularly good event as it leaves a long track in the detector enabling a good measurement of the direction from timing. Looking at the time distribution of track hits with respect to the reconstructed $y$ position of the hit, it is clear that this event is travelling upwards through the detector. Due to the low momentum of the muon it is possible to measure the momentum and charge with a high degree of confidence, as their is significant curvature in the magnetic field. The least squares fit to the curvature determines that this event contains a $\mu^{-}$with a momentum of $5.3 \pm 0.1 \mathrm{GeV}$.

Another example event is shown in Figure 9.12. As with the previous event the time distribution of track hits with respect to the reconstructed $y$ position of the hits clearly indicate that the particle is travelling upwards through the detector. The muon enters through the first plane of the detector, does not stop and leaves through the top of the detector. The fact that the muon does not stop in the detector gives an indication that it is a more energetic particle than the one shown in the previous event. Further evidence of this fact is available through the minimal curvature of the track in the magnetic field. The fit reports that the particle is a $\mu^{+}$ with momentum of $38 \pm 4 \mathrm{GeV}$, even at this energy the Monte Carlo studies suggest that the charge of the particle is correctly identified in over $90 \%$ of the events.

Before cutting on the slope of time distribution with respect to the $y$ position a sample of 618143 muon events with good timing information remain. Of these events only 10 are upward-going muons with a positive slope to the time gradient in the $y$ plane and no obvious misreconstruction. The expected number of events for a sample of this size is 12 , with a conservatively set $30 \%$ systematic uncertainty due mainly to the uncertainty in overall atmospheric neutrino flux. Within $1 \sigma$ the 

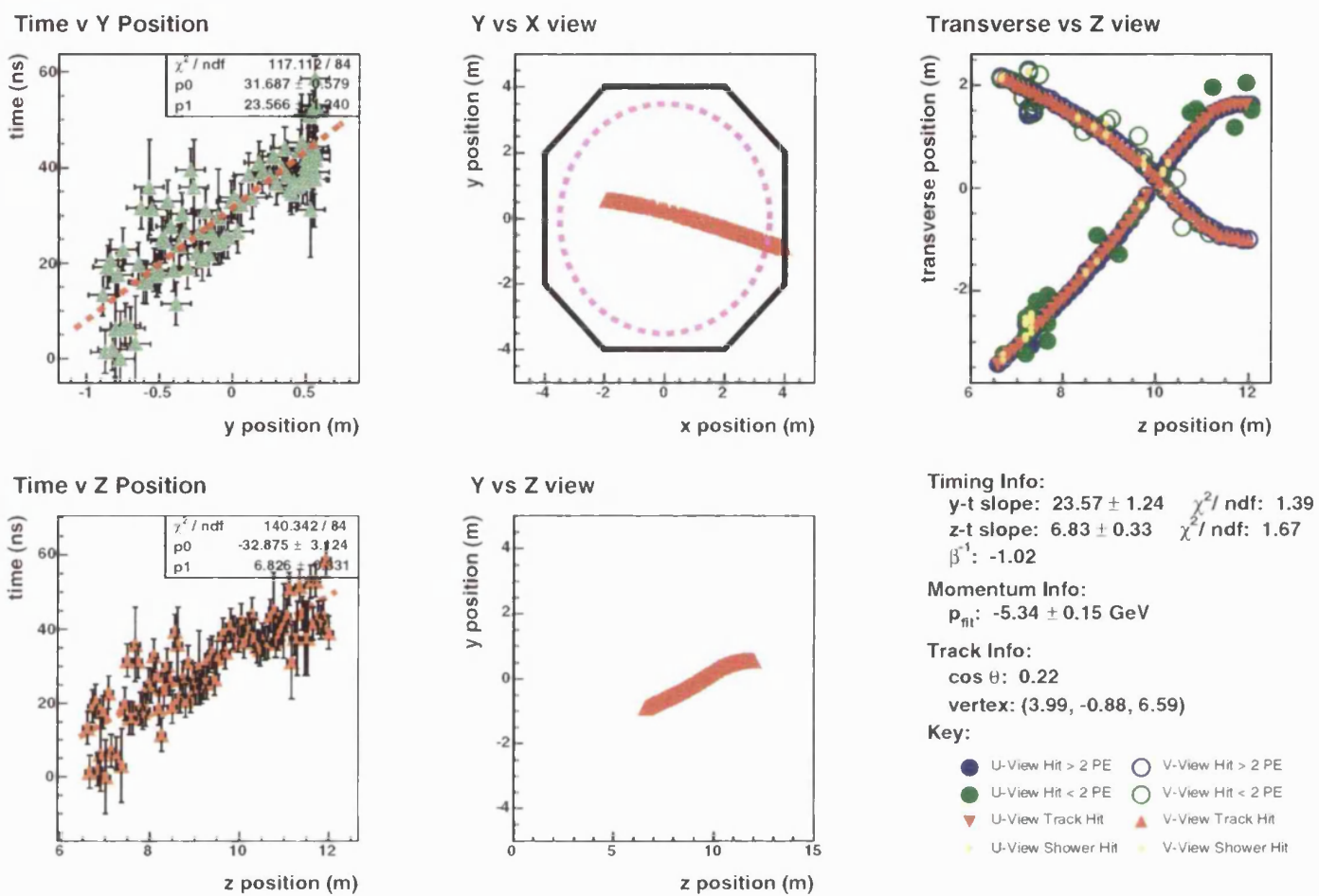

Figure 9.11: An event display showing upward-going muon in the MINOS Far Detector. The top righthand plot shows all of the hits in the event in terms of transverse and longitudinal position as measured directly by the location of the scintillator strips. The majority of the large (greater than two photoelectrons) hits lie along the path of the reconstructed track, the smaller hits that surround the track are predominantly the result of crosstalk in the M16 PMTs. The middle two plots show the three dimensional reconstructed position of the tracked hits in terms of $x-y$ and $y-z$ projections, with the edges of the detector and the fiducial volume shown on the $x-y$ projection. The two leftmost plots show the slope of the time gradient with respect to the $y$ and $z$ positions. Looking at the timing distributions and event topology this event is clearly identifiable as an upward-going muon which enters the detector through the righthand side as displayed in the $x-y$ projection 

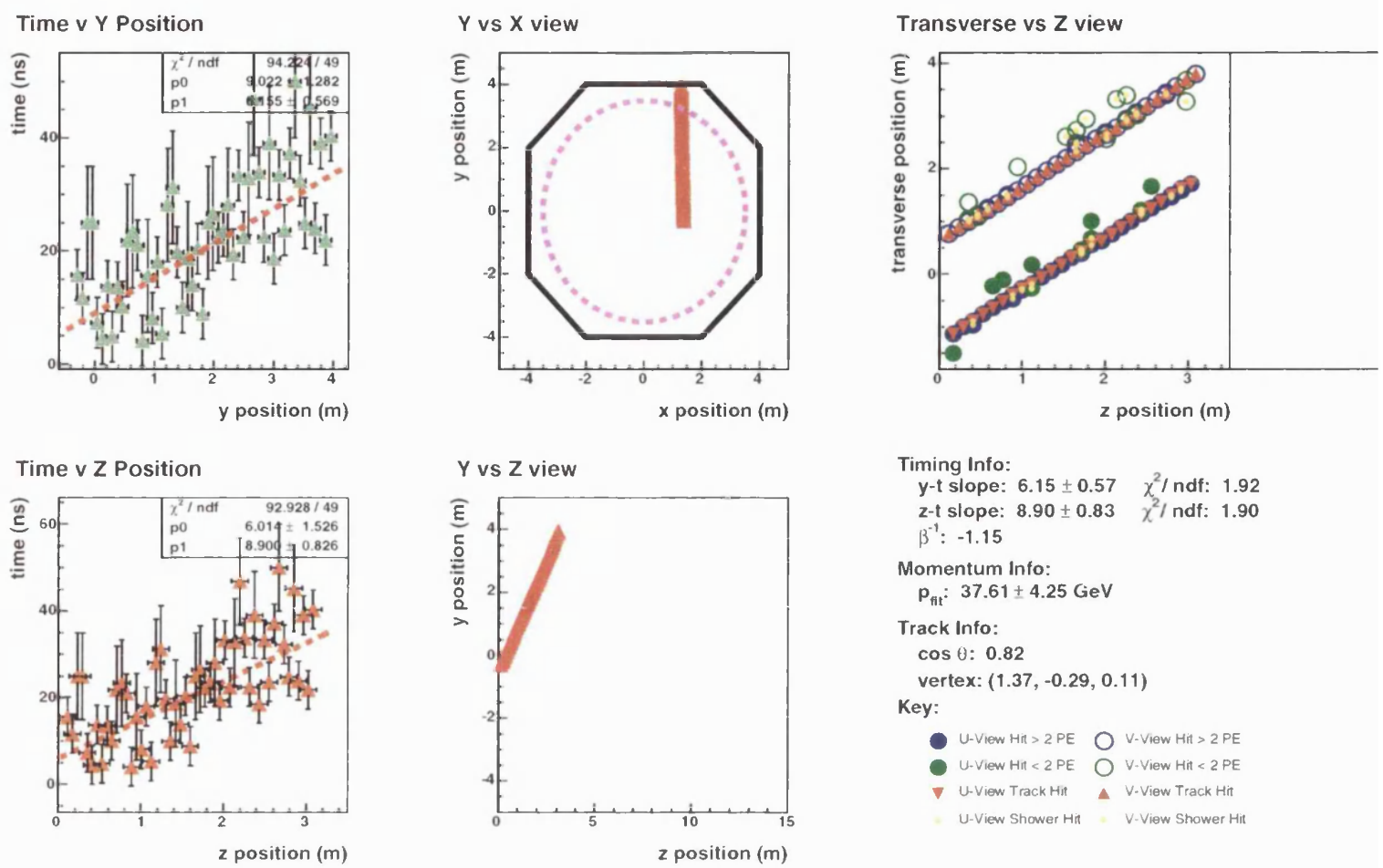

Figure 9.12: An event display of one of the 10 upward-going muon events identified in the sample. Unlike the previous event, shown in Figure 9.11, this event contains a relatively high energy muon which does not stop in the detector. Due to the larger moment um of the muon the track only curves slightly in the magnetic field. The track is inclined at a much steeper angle to the horizontal than the previous event. From the timing distributions it is clear that the muon entered the detector through its front face and left through the top of the detector.

measured and predicted numbers are in agreement.

A good test of the timing resolution of the detector is to calculate the velocity of the muon using the time gradient and the distance travelled through the detector. The $\frac{1}{\beta}$ distribution, where $\beta=\frac{v}{c}$, is plotted in Figure 9.13. The downwards going cosmic ray muons are in the large peak centred at 1 , the value expected for relativistic particles. The timing resolution can be inferred from the $11 \%$ width of the distribution. Also visible is the small peak at -1 which corresponds to the upward-going muons.

The standard way in which muons induced from atmospheric neutrinos are used 


\section{Velocity Distribution}

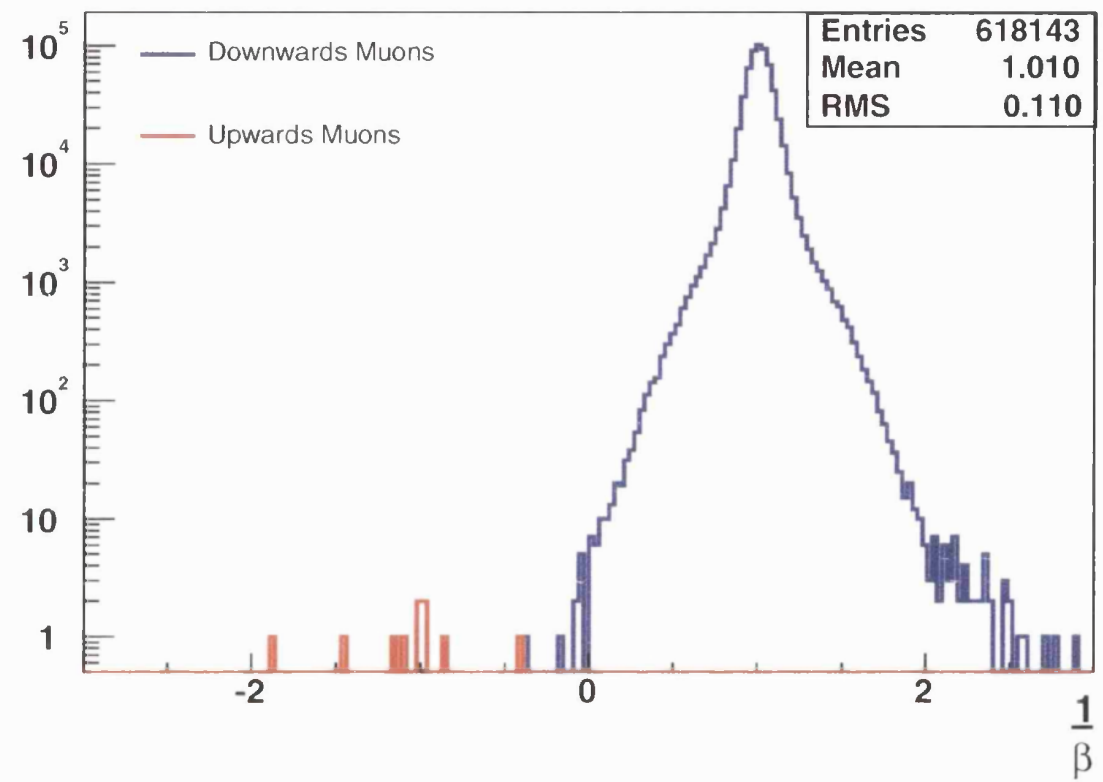

Figure 9.13: The velocity distribution of muons in the Far Detector, in terms of $\beta=\frac{v}{c}$. The events in the large blue peak are downwards going muons, as one would expect for relativistic particles the peak is centred at 1 . The $11 \%$ width of the distribution is a measure of the timing resolution of the detector. The small red peak at -1 is due to upward-going muons.

to measure the oscillation parameters is through examining the flux as a function of vertical angle. This is a powerful tool as the amount of material, and hence the baseline in the oscillation equation, changes with the vertical angle. Muons travelling vertically through the detector most likely come from neutrinos which have travelled across almost the entire diameter of the Earth, whilst those travelling almost horizontally have travelled through much less material. This variation of baseline with angle is illustrated in Figure 3.7, with regards to the Super-Kamiokande experiment and its atmospheric neutrino measurements. Therefore the flux of neutrinos with and without oscillations will vary as a function of vertical angle. With so few events it is not possible to make a measurement of the neutrino flux as a function of angle to test oscillation hypotheses. However for completeness the flux of upward-going 
muons as a function of angle is shown in Figure 9.14. Also shown is the predicted reconstructed flux, in the absence of neutrino oscillations. With so few events it is not possible to determine whether or not there is any disagreement with the predicted flux.

\section{Angular Distribution of Upwards Going Muons}

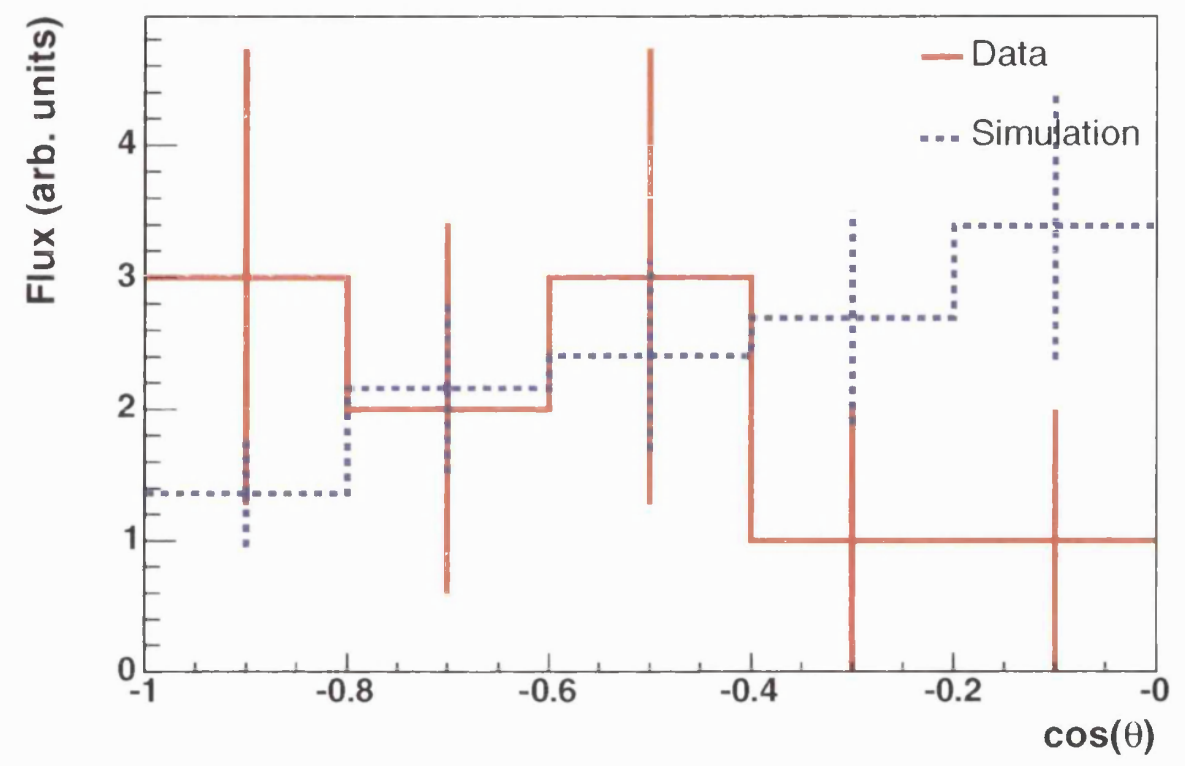

Figure 9.14: The flux of neutrino induced upward-going muons as a function of vertical angle. In this plot $\cos \theta=-1$ corresponds to a muon travelling vertically through the detector and $\cos \theta=0$ to a muon travelling horizontally. The data points are from the 10 upward-going muon events detected, with purely statistically error bars. The simulation results are from a Nuance simulation by B. Rebel [76][78], the error bars on the simulation are a conservative estimate of $30 \%$ systematic uncertainty, coming primarily from the overall neutrino flux.

Where MINOS is different from all previous atmospheric neutrino experiments is in the charge determination capabilities due to the magnetic field. This capability will allow MINOS to make a number of interesting measurements that have not been possible until now. The simplest of these is a measurement of the charge distribution of atmospheric neutrino induced muons. Due to differences in the initial flux and interaction cross-sections the expected ratio of $\mu^{-}: \mu^{+}$is approximately $2: 1$. The 
momentum and charge distribution of 8 upward-going muon events is plotted in Figure 9.15, the fit failed to converge for the other two events. When all 8 of these events are considered the ratio $\mu^{-}: \mu^{+}$is $3: 5$. Limiting the sample to only those 6 muons whose momentum is less than $50 \mathrm{GeV}$, below which the charge identification is correct at better than the $90 \%$ level, leaves $3 \mu^{-}$and $3 \mu^{+}$events. 


\section{Momentum Distribution of Upwards Going Muons}

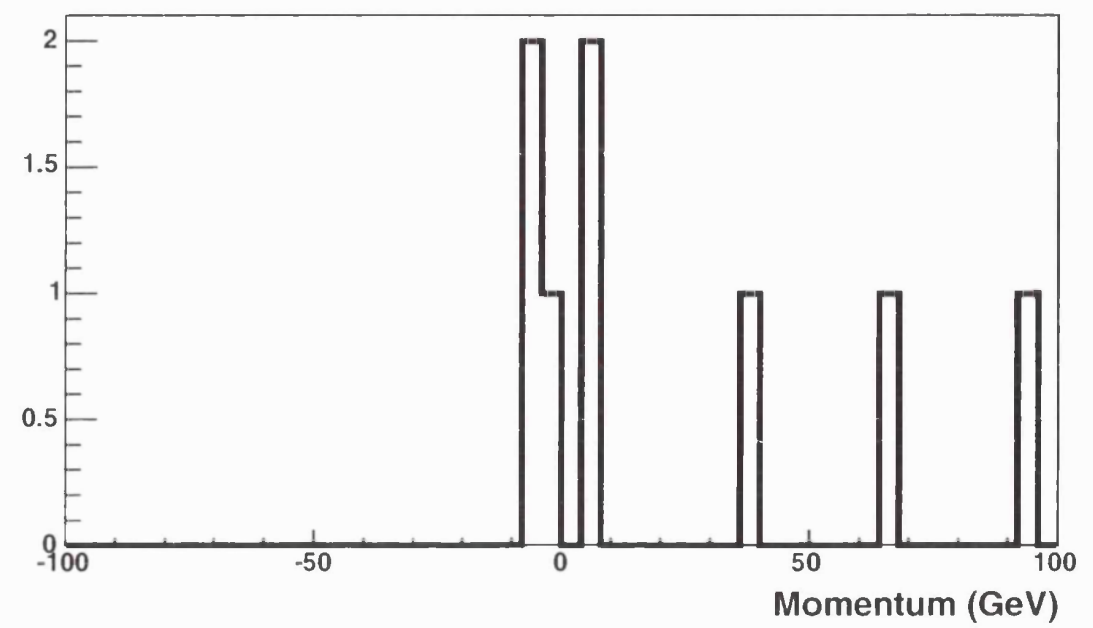

\section{Charge Distribution of Upwards Going Muons}

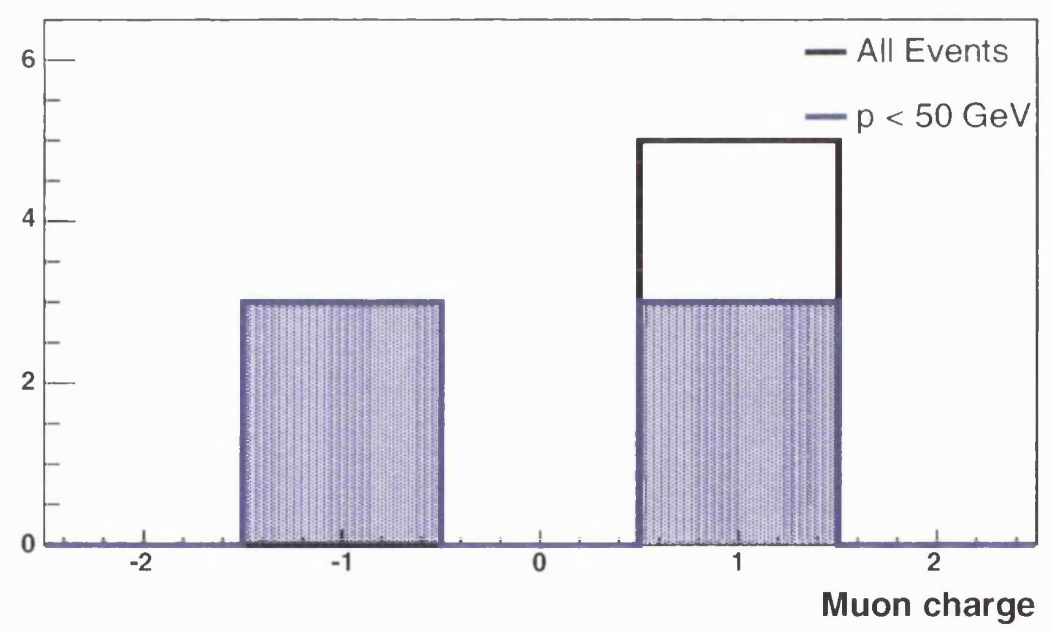

Figure 9.15: The charge and momentum distribution of a sample of 8 neutrino induced upward-going muons in the MINOS Far Detector. When all events are considered the ratio of $\mu^{-}$to $\mu^{+}$is $3: 5$. If the sample is limited to those muons whose momentum is measured to be less than $50 \mathrm{GeV}$, below which the charge is correctly identified at the $90 \%$ level, the ratio $\mu^{-}: \mu+$ is $3: 3$. This ratio is expected to be $2: 1$ in favour of the $\mu^{-}$. 


\subsection{Summary}

In terms of atmospheric neutrino studies, one of the most interesting features of the large MINOS detectors is their magnetization. This will enable MINOS to measure the response of $\nu_{\mu}$ and $\bar{\nu}_{\mu}$ separately, identifiable by the charge of the muon produced in the interaction. The charge can be determined through the curvature of the muon track in the magnetic field.

An implementation of the CDHS least squares method for fitting muon tracks has been developed for the MINOS detectors. Using this track fitting algorithm it is possible to determine the charge and momentum of muons in the detector from the curvature of their tracks in the magnetic field. The method includes a realistic treatment of errors due to multiple scattering, energy loss and position measurement uncertainty. From Monte Carlo studies the charge identification of muons has been shown to be better than $90 \%$ for particles of momentum below $50 \mathrm{GeV}$. Above $100 \mathrm{GeV}$ there is very little curvature in the magnetic field and as such only lower bounds on the muon momentum can be set and essentially there is no charge separation capability.

A sample of data from 102 days of livetime with one magnetized super-module of the MINOS Far Detector has been used to search for neutrino induced upwardgoing muons. A total of 10 events have been identified, the expectation based upon simulations performed by B. Rebel [76] is for there to be 12 events. Both the angular flux and the charge distribution of these events have been investigated. The measured flux is consistent with the predicted flux with no oscillations, although the statistics are not large enough to disfavour any oscillation models. The ratio of $\mu^{-}: \mu^{+}$which is expected to be $2: 1$ is measured at $1: 1$ for muons with momentum less than $50 \mathrm{GeV}$, using the least squares method described, and as such agrees with the predicted ratio within $1 \sigma$. With such scant statistics it is impossible to draw any definitive conclusions beyond the fact that MINOS is capable of identifying upwardgoing muons in the Far Detector through timing and event topology. Over the course of the next 5 years enough statistics will be obtained such that competitive measurements utilising the charge separation capabilities of the magnetic field can 
be made. 


\section{Chapter 10}

\section{Conclusions}

MINOS is a long baseline neutrino oscillation experiment that is expected to start taking beam data at the end of 2004. The main measurement of the oscillation parameters will come from a comparison of the neutrino energy spectrum measured with the Far Detector to the predicted flux based upon measurements made with the Near Detector. Before this comparison is relevant the responses of the two detectors must be calibrated relative to each other. The detectors have been designed to achieve a relative calibration of the Near and Far Detector energy scales to $2 \%$ and an absolute calibration of the energy scale to $5 \%$. The relative calibration will be performed using muons and the absolute energy scale using data from testbeams taken with the Calibration Detector.

The first stage in the relative calibration is to ensure that the response of each strip in a given detector is the same at any point in time. This is achieved using a light injection system to remove short timescale variations in the response of the PMTs and electronics. At the Calibration Detector variations of up to $40 \%$ have been shown to be calibrated to the $1 \%$ level. Variations in the response of scintillator strips are removed using the spectrum generated by muon energy deposits in the strips. At the Calibration Detector variations of up to $30 \%$ in the response of the scintillator strips and optical readout cables have been calibrated to better than $2 \%$ using samples of both cosmic ray and beam related muons.

After the intra-detector responses have been calibrated the MEU scales of the 
three MINOS detectors must be calibrated relative to each other. Muons of known energy are used for this calibration. A method for calibrating the energy scale of the detectors using stopping muons has been presented. This method has been used to calibrate the MEU scale of the Calibration Detector, between the T11 and T7 testbeam enclosures, to the $2 \%$ level. The feasibility of a cross-detector calibration between the Calibration and Far Detectors using this method has been presented.

An implementation of the CDHS least squares method for fitting the curvature of a muon track in a magnetic field has been developed for the MINOS detectors. Using the curvature it is possible to determine both the momentum and charge of the muons. This method has been tested using both simulated and real cosmic ray muon data from the Far Detector. From the simulated data the efficiency of charge determination is shown to be better than $95 \%$ for those muons measured to have momentum less than $50 \mathrm{GeV}$.

Using this track fitter an initial measurement of the charge distribution of atmospheric neutrino induced upward-going muons has been made. Using a data sample corresponding to 102 days of live time 10 upward-going muons events have been identified. The expected number of events for a data sample of this amount of time is 12 events. Due to differences in the the cross-sections for neutrino and anti-neutrino interactions with matter the ratio of $\mu^{-}: \mu^{+}$is expected to be approximately $2: 1$. Using all 8 muons for which the fit converged the ratio is measured to be 3:5, restricting the sample to just those events whose momentum is measured to be less than $50 \mathrm{GeV}$ the ratio is $3: 3$. Within statistical errors this measurement is in agreement with the predicted ratio of $2: 1$.

Over the course of the next 5 years the MINOS experiment will be able to make a number of interesting measurements in the field of neutrino physics. Among these will be measurements of the oscillation parameters for $\nu_{\mu}$ and $\bar{\nu}_{\mu}$ separately using the charge separation capabilities of the magnetized detectors. 


\section{Bibliography}

[1] W. Pauli, Letter reproduced in translation, Physics Today (1978).

[2] F. Reines and C. L. Cowan, Phys. Rev. 92, 830 (1953).

[3] C. S. Wu, E. Ambler, R. W. Hayward, D. D. Hoppes, and R. P. Hudson, Phys. Rev. 105, 1413 (1957).

[4] M. Goldhaber, L. Grodzins, and A. W. Sunyar, Phys. Rev. 109, 1015 (1958).

[5] B. Adeva et al. (L3), Z. Phys. C51, 179 (1991).

[6] G. Alexander et al. (OPAL), Z. Phys. C52, 175 (1991).

[7] P. Abreu et al. (DELPHI), Nucl. Phys. B367, 511 (1991).

[8] D. Decamp et al. (ALEPH), Z. Phys. C53, 1 (1992).

[9] J. N. Bahcall, M. H. Pinsonneault, and S. Basu, Astrophys. J 555, 990 (2001).

[10] Q. R. Ahmad et al. (SNO), Phys. Rev. Lett. 89, 011301 (2002).

[11] Y. Fukuda et al. (Super-Kamiokande), Phys. Rev. Lett. 81, 1158 (1998).

[12] Y. Fukuda et al. (Super-Kamiokande), Phys. Lett. B 433, 9 (1998).

[13] B. Pontecorvo, JETP 33, 549 (1957).

[14] B. Pontecorvo, JETP 34, 247 (1958).

[15] B. Pontecorvo, JETP 53, 1717 (1967).

[16] K. Eguchi et al. (KamLAND), Phys. Rev. Lett. 90, 021802 (2003). 
[17] H. V. Klapdor-Kleingrothaus, A. Dietz, H. L. Harney, and I. V. Krivosheina, Mod. Phys. Lett. A16, 2409 (2001).

[18] L. Wolfenstein, Phys. Rev. D17, 2369 (1978).

[19] S. P. Mikheev and A. Y. Smirnov, Sov. J. Nucl. Phys. 42, 913 (1985).

[20] S. P. Mikheev and A. Y. Smirnov, Nuovo Cim. C9, 17 (1986).

[21] R. D. Jr., D. S. Harmer, and K. C. Hoffmann, Phys. Rev. Lett. 20, 1205 (1968).

[22] J. N. Bahcall, URL http://www.sns.ias.edu/ jnb/.

[23] A. I. Abazov et al. (SAGE), Phys. Rev. Lett. 67, 3332 (1991).

[24] D. N. Abdurashitov et al. (SAGE), Phys. Rev. Lett. 77, 4708 (1996).

[25] P. Anselmann et al. (GALLEX), Phys. Lett. B 285, 376 (1992).

[26] W. Hampel et al. (GALLEX), Phys. Lett. B 388, 384 (1996).

[27] M. Altmann et al. (GNO), Phys. Lett. B 490, 16 (2000).

[28] K. S. Hirata et al. (Kamiokande-II), Phys. Rev. Lett. 65, 1297 (1990).

[29] Y. Fukuda et al. (Super-Kamiokande), Phys. Rev. Lett. 82, 1810 (1999).

[30] S. Fukuda et al. (Super-Kamiokande), Phys. Rev. Lett. 86, 5656 (2001).

[31] Q. R. Ahmad et al. (SNO), Phys. Rev. Lett. 89, 011302 (2002).

[32] S. N. Ahmed et al. (SNO) (2003), nucl-ex/0309004.

[33] P. Adamson, Ph.D. thesis, Univeristy of Sussex (2001).

[34] R. Becker-Szendy et al., Phys. Rev. Lett. 69, 1010 (1992).

[35] R. Becker-Szendy et al., Nucl. Phys. Proc. Suppl. 38, 331 (1995).

[36] S. Hatakeyama et al., Phys. Rev. Lett. 81, 2016 (1998). 
[37] Y. Fukuda et al. (Super-Kamiokande), Phys. Rev. Lett. 81, 1562 (1998).

[38] M. Aglietta et al., Europhys. Lett. 8, 611 (1989).

[39] K. Daum et al., Z. Phys. C 66, 417 (1995).

[40] W. W. M. Allison et al., Phys. Lett. B 449, 137 (1999).

[41] C. Yanagisawa, Nucl. Phys. Proc. Suppl. 95, 93 (2001).

[42] M. Apollonio et al. (CHOOZ), Phys. Lett. B 466, 415 (1999).

[43] L. Ludovici, Nucl. Phys. Proc. Suppl. 91, 177 (2001).

[44] P. Astier et al. (NOMAD), Nucl. Phys. B 611, 3 (2001).

[45] C. Athanassopoulos et al. (LSND), Phys. Rev. Lett. 81, 1774 (1998).

[46] H. Gemmeke et al., Nucl. Inst. Meth. A 289, 490 (1990).

[47] A. O. Bazarko (BooNe) (1999), hep-ex/9906003.

[48] K. Nakamura (K2K), Nucl. Phys. Proc. Suppl. 91, 203 (2001).

[49] M. H. Ahn et al. (K2K), Phys. Rev. Lett. 90, 041801 (2003).

[50] D. Duchesneau (OPERA) (2002), hep-ex/0209082.

[51] K. Hagiwara et al., Physical Review D 66, 010001+ (2002), URL http://pdg.lbl.gov.

[52] The MINOS Collaboration, "The NuMI Technical Design Handbook." (1999).

[53] The MINOS Collaboration, "The MINOS Technical Design Report.", NuMINOTE-337 (1999).

[54] The MIPP Collaboration, URL http://ppd.fnal.gov/experiments/e907/e907.htm.

[55] P. Adamson, A. Para, and J. Thomas, "Performance of a least-squares based muon reconstruction procedure for MINOS.", NuMI-NOTE-0536. 
[56] C. Smith, Ph.D. thesis, Univeristy College London (2002).

[57] J. Cobb, "A test for Neutrino Oscillations Using the Energy Distributions of Numu Charged Current Events.", NuMI-NOTE-0106.

[58] D. Petyt, Ph.D. thesis, Univeristy of Oxford (1998).

[59] D. Petyt,

URL http://webusers . physics.umn. edu/ petyt/cc/30_07_03/.

[60] P. Adamson et al., Nucl. Instrum. Meth. A492, 325 (2002).

[61] V. Abramov et al., "Study of magnetic field influence on hadron calorimeter response.", IFVE-96-91.

[62] S. Eilerts et al., "Effects of Magnetic Fields on Hamamatsu R599-00-M16 PMTs.", NuMI-NOTE-0568.

[63] M. Barker et al., "Effects of Magnetic Fields on Hamamatsu R5900-00-M64 PMTs.", NuMI-NOTE-0602.

[64] M. Barker et al., "A More Rigorous Description of the Influence of Magnetic Fields on Hamamatsu M64 Photomultipliers in Relation to the MINOS Experiment.", NuMI-NOTE-0650.

[65] P. Adamson et al., "Photoelectron Counting by Several Methods.", NuMINOTE-0661.

[66] H. Kim, private communication.

[67] J. Liu et al., "Study of gain non-uniformity of M16 PhotoMultiplier.", NuMINOTE-0797.

[68] P. Harris et al., to be published.

[69] C. Smith and R. Nichol, "Path-Length Corrections and Zero Reconstruction in the MINOS Detectors.", NuMI-NOTE-0971. 
[70] R. Lee and S. Seun, "Calibration of MINOS detectors using Cosmic Ray Muons.", NuMI-NOTE-0694.

[71] S. Wojicki, private communication.

[72] P. Vahle et al., "Electromagnetic Interactions in the MINOS Calibration Detector.", NuMI-NOTE-0945.

[73] J. Thomas, A. Para, and D. Tovee, "The Principles of Muon tracking in MINOS.", NuMI-NOTE-0301.

[74] R. Lee, "A Description of the MINOS Reconstruction Framework.", NuMINOTE-0916.

[75] D. DeMuth et al. (2003), hep-ex/0304016.

[76] B. Rebel, "Upward-Going Muon Analysis with the MINOS Far Detector.", American Physical Society Meeting (2003).

[77] R. Lee, "Up Going Muons.", Internal MINOS talk.

[78] D. Casper, "Simulation of upward-going muons." (1998). 